\title{
WestVirginiaUniversity
}

THE RESEARCH REPOSITORY @ WVU

Graduate Theses, Dissertations, and Problem Reports

2004

\section{Linear engine development for series hybrid electric vehicles}

\author{
Csaba Toth-Nagy \\ West Virginia University
}

Follow this and additional works at: https://researchrepository.wvu.edu/etd

\section{Recommended Citation}

Toth-Nagy, Csaba, "Linear engine development for series hybrid electric vehicles" (2004). Graduate Theses, Dissertations, and Problem Reports. 2642.

https://researchrepository.wvu.edu/etd/2642

This Dissertation is protected by copyright and/or related rights. It has been brought to you by the The Research Repository @ WVU with permission from the rights-holder(s). You are free to use this Dissertation in any way that is permitted by the copyright and related rights legislation that applies to your use. For other uses you must obtain permission from the rights-holder(s) directly, unless additional rights are indicated by a Creative Commons license in the record and/ or on the work itself. This Dissertation has been accepted for inclusion in WVU Graduate Theses, Dissertations, and Problem Reports collection by an authorized administrator of The Research Repository @ WVU.

For more information, please contact researchrepository@mail.wvu.edu. 


\title{
Linear Engine Development for Series Hybrid Electric Vehicles
}

\author{
Csaba Tóth-Nagy
}

Dissertation submitted to the College of Engineering and Mineral Resources at

West Virginia University

in partial fulfillment of the requirements for the degree of

Doctor of Philosophy

in the field of

Mechanical Engineering

Examining committee:

Nigel N. Clark Ph.D., Chair Christopher M. Atkinson Sc.D.

Larry E. Banta Ph.D.

Parviz Famouri Ph.D.

Gregory J. Thompson Ph.D.

W. Scott Wayne Ph.D.

Department of Mechanical and Aerospace Engineering

Morgantown, WV

2004

Keywords: Linear engine, Free piston engine, Linear alternator, Compression ignition, Prototype, Simulation, Neural network, Genetic algorithm, Hybrid electric vehicle

Copyright 2004 Csaba Tóth-Nagy 


\section{Abstract \\ Linear Engine Development for Series Hybrid Electric Vehicles \\ by}

\section{Csaba Tóth-Nagy}

This dissertation argues that diminishing oil reserves, concern over global climate change, and desire to improve ambient air quality all demand the development of environment-friendly personal transportation. In certain applications, series hybrid electric vehicles offer an attractive solution to reducing fuel consumption and emissions. Furthermore, linear engines are emerging as a powerplant suited to series HEV applications. In this dissertation, a linear engine/alternator was considered as the auxiliary power unit of a range extender series hybrid electric vehicle. A prototype linear engine/alternator was developed, constructed and tested at West Virginia University. The engine was a 2-stroke, 2-cylinder, dual piston, direct injection, diesel engine. Experiment on the engine was performed to study its behavior. The study variables included mass of the translator, amount of fuel injected, injection timing, load, and stroke with operating frequency and mechanical efficiency as the basis of comparison. The linear engine was analyzed in detail and a simple simulation model was constructed to compare the trends of simulation with the experimental data and to expand on the area where the experimental data were lacking. The simulation was based on a simple and analytical model, rather than a detailed and intensely numerical one. The experimental and theoretical data showed similar trends. Increasing translator mass decreased the operating frequency and increased compression ratio. Larger mass and increased compression ratio improved the ability of the engine to sustain operation and the engine was able to idle on less fuel injected into the cylinder. Increasing the stroke length caused the operating frequency to drop. Increasing fueling or decreasing the load resulted in increased operating frequency. This projects the possibility of using the operating frequency as an input for feedback control of the engine. Injection timing was varied to investigate two different modes of engine operation experimentally. The two modes were direct injection compression ignition (DICI) and "pseudo" homogeneously charged compression ignition (PHCCI). Simulation was performed to include HCCI operation in the study. The study showed that the HCCI operation resulted in higher peak cylinder pressure than that of DICI operation. A combined genetic algorithm-artificial neural network predictor model was used along with the simulation model to find the combination of engine parameters that yielded the highest engine efficiency. The predictor-simulator model suggested the most efficient combination of engine parameters. 
Table 1 shows the parameters of the predicted most efficient engine and the prototype engine with dimensions as well as dimensionless parameters.

Table 1. The combination of engine parameters that yielded the highest mechanical efficiency. Bore, effective stroke, total stroke, fuel mass, load force, and injection timing were the input parameters to the predictor/simulator; operating frequency, compression ratio, mechanical efficiency, and output power were the simulated parameters.

\begin{tabular}{|l|r|r|}
\hline & Simulated best efficiency engine & Prototype engine \\
\hline Bore (mm) & 108.5 & 76 \\
\hline Effective stroke (mm) & 27.140 & 38.000 \\
\hline Total stroke (mm) & 40.710 & 73.800 \\
\hline Fuel mass (g/injection) & 0.0074760 & 0.0031080 \\
\hline Load force (N) & 3970 & 138 \\
\hline Injection timing (mm before TDC) & 24.4 & 10 \\
\hline Operating frequency (Hz) & 116 & 53 \\
\hline Compression ratio & 13.12 & $\mathrm{na}$ \\
\hline Mechanical efficiency (\%) & 50.43 & 11 \\
\hline Power output (W) & 37,500 & 1080 \\
\hline
\end{tabular}




\section{Dedication}

To my father, who introduced me to engines. 


\section{Acknowledgements}

I thank Dr. Nigel N. Clark for his guidance during my postgraduate studies. Not only he did provide invaluable input to my research, he also showed the way with his integrity, philosophy, and attitude. I also thank Dr. Christopher M. Atkinson, Dr. Larry E. Banta, Dr. Parviz Famouri, Dr. Gregory J. Thompson, and Dr. W. Scott Wayne for serving on my Ph.D. committee and for providing important feedback. Thanks to Dr. Thompson for providing room for the linear engine in the Engine and Emissions Research Center (EERC). I also thank the staff of the EERC for their support. Thanks to Tom Spencer for organizing the logistics of the EERC, Richard Atkinson for his knowledge that he was always willing to share, Dan Carder for knowing about everything in the facility, Andy Pertl for providing the data acquisition program for this study. Special thanks to Hasan Muhammad and Ezekiel Turak for their dedicated work on the

linear engine. And last but not least I thank the US Army and Dr. David Mann for the funding of the linear engine/alternator project (Contract \#: DAAD19-01-041). 


\section{Table of contents}

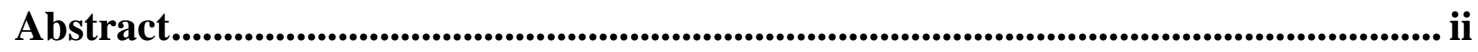

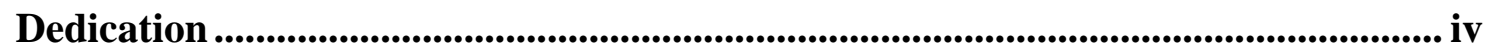

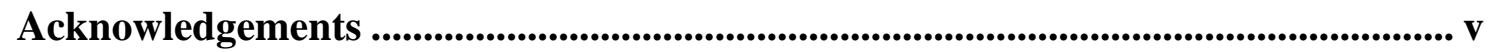

Table of contents ................................................................................................ vi

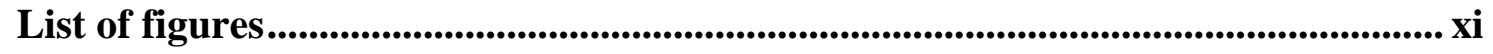

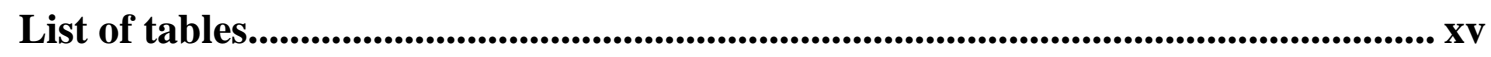

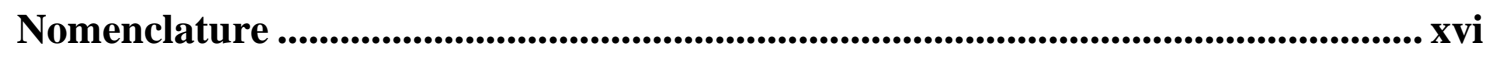

Objectives.............................................................................................................................. 1

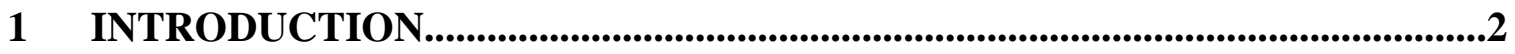

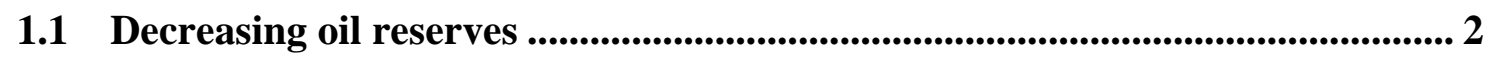

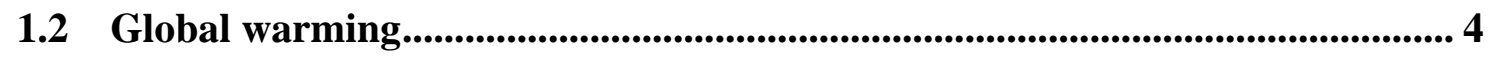

1.3 Corporate average fuel economy (CAFE) standards ................................. 9

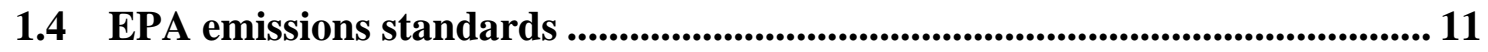

1.5 Conclusions ............................................................................................................. 14

2 HYBRID ELECTRIC VEHICLES .............................................................15

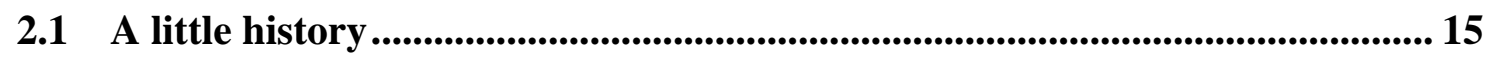

2.2 Hybrid configurations ............................................................................. 17

2.2.1 Advantages and disadvantages of conventional vehicles ........................... 17

2.2.2 Advantages and disadvantages of electric vehicles .................................. 18

2.2.3 Advantages and disadvantages of series HEVs ......................................... 20

2.2.4 Advantages and disadvantages of parallel HEVs ...................................... 23

2.2.5 Advantages and disadvantages of combination HEVs ............................... 25

2.2.6 Advantages and disadvantages of fuel cell hybrids .................................. 29

2.3 Benefits and drawbacks of hybridization ........................................................... 30

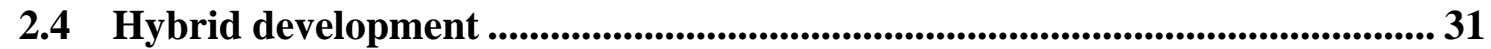

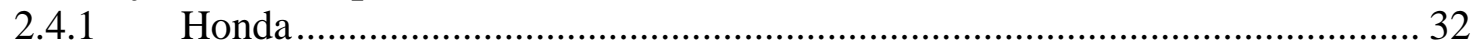

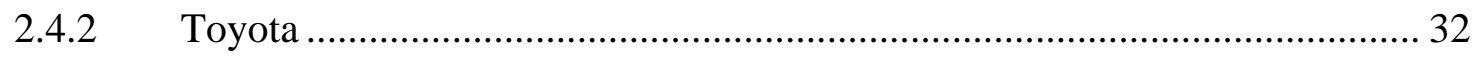




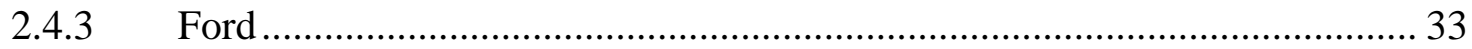

2.4.4 General Motors (GM ) .............................................................................. 33

2.4.5 Hybrid electric high mobility multipurpose wheeled vehicle (HHMMWV) 33

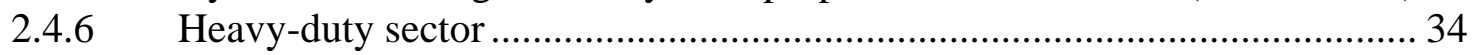

2.5 Conclusions ...................................................................................................................... 34

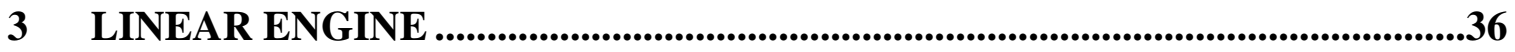

3.1 The first attempts ........................................................................................................ 37

3.2 External combustion versus internal combustion................................................. 38

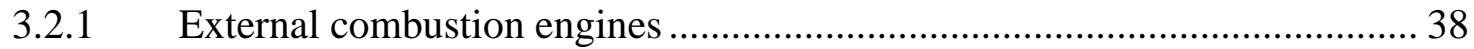

3.2.2 Internal combustion ...................................................................................... 44

3.3 Spark ignition versus compression ignition............................................................. 45

3.4 Direct injection versus homogeneously charged compression ignition.......... 47

3.5 Four stroke versus two stroke........................................................................50

3.6 Number of cylinders and configuration................................................................. 53

$3.7 \quad$ Simulation of the linear engine .....................................................................59

3.8 Applications of linear engines ............................................................................6 60

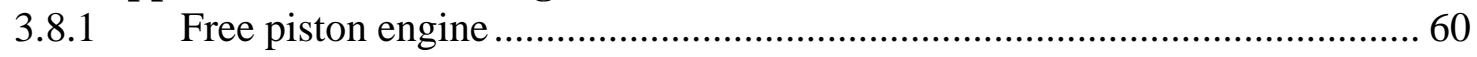

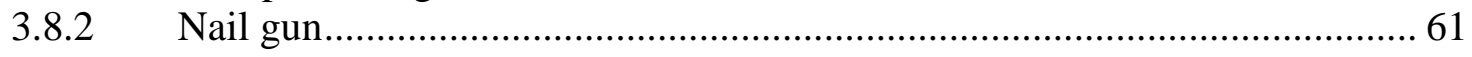

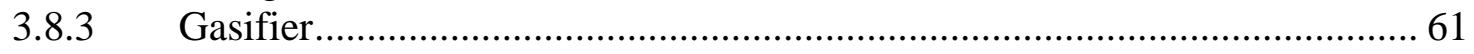

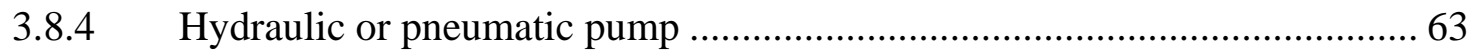

3.8.5 Electric generator ......................................................................................... 65

3.9 Conclusions .................................................................................................................... 68

4 THEORETICAL ANALYSIS.....................................................................................70

4.1 Analysis of the linear engine ................................................................................... 70

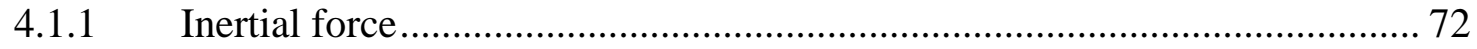

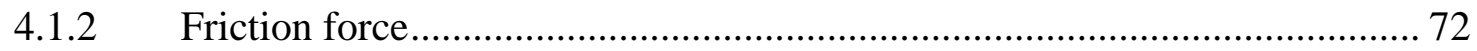

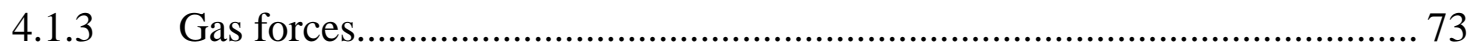

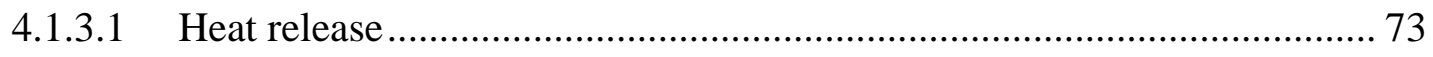

4.1.3.2 Scavenging air pressure ....................................................................... 75

4.1.4 Load force ……............................................................................... 76

4.2 Dimensional analysis............................................................................................. 77

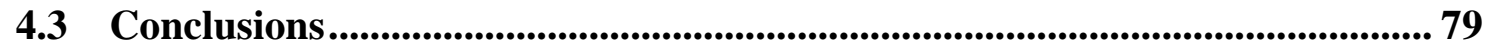




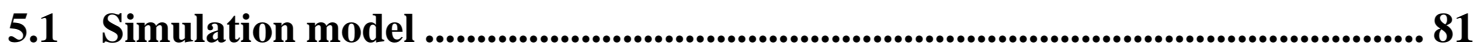

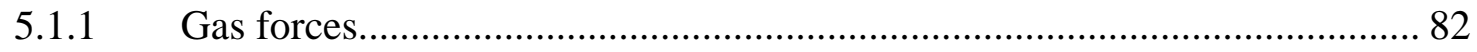

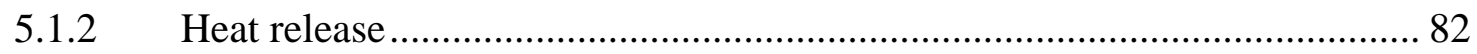

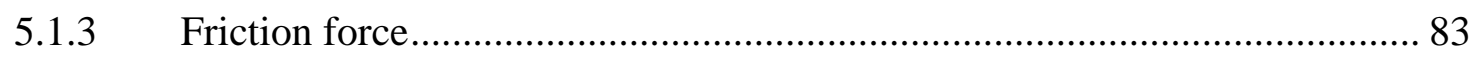

5.2 The predictor/simulator model ................................................................ 85

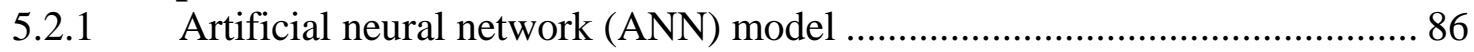

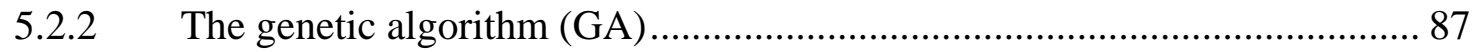

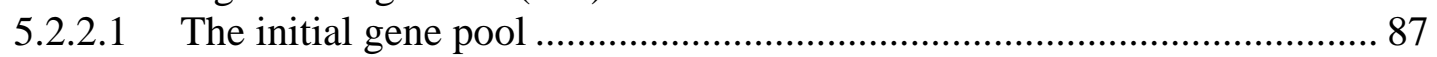

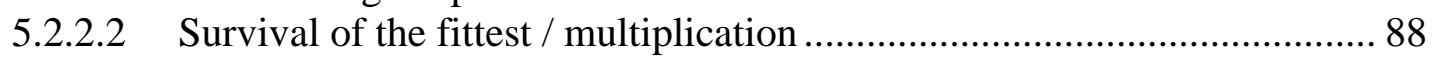

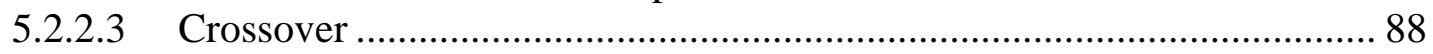

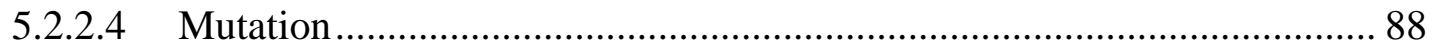

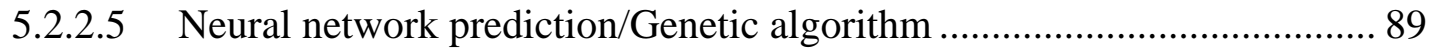

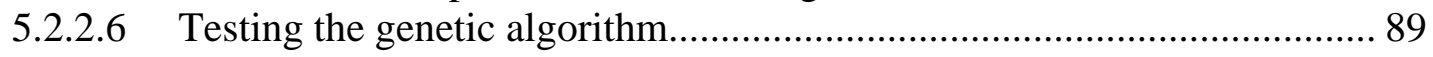

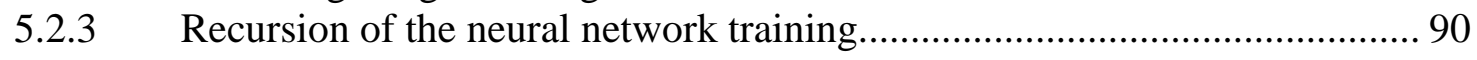

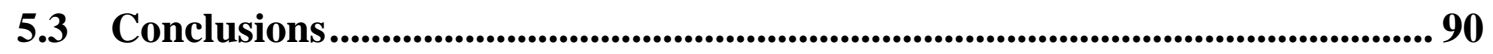

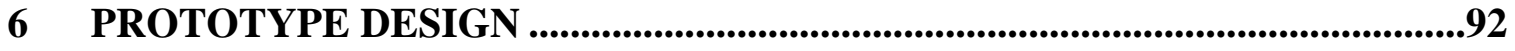

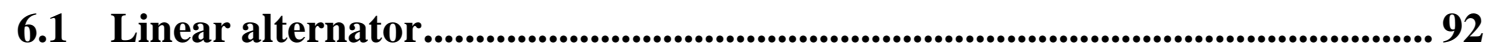

6.2 Linear engine ..................................................................................................... 93

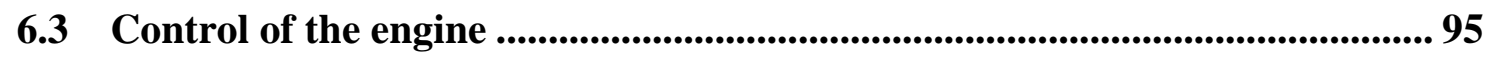

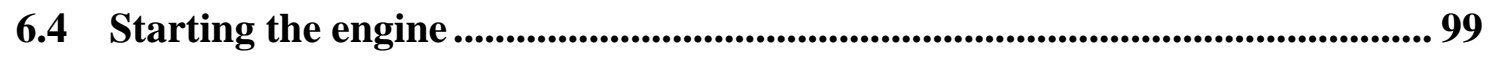

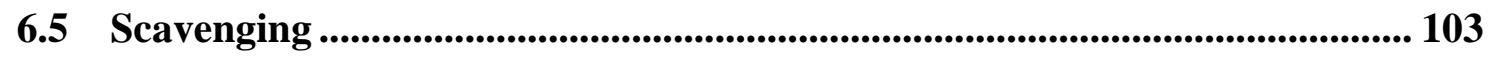

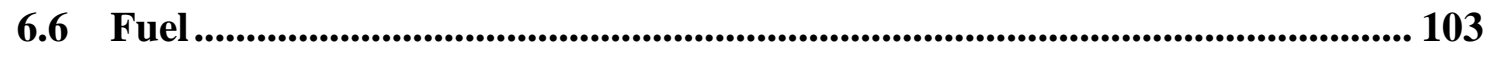

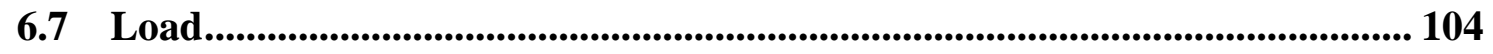

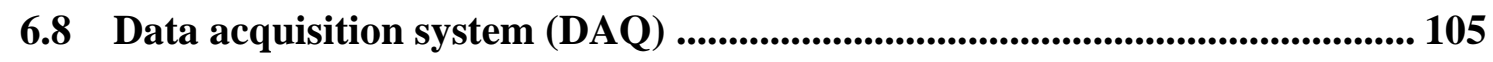

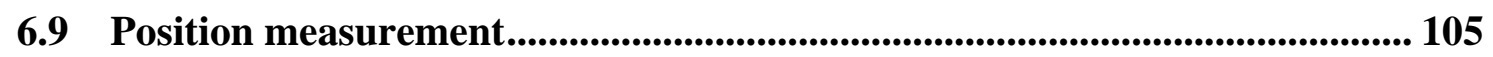

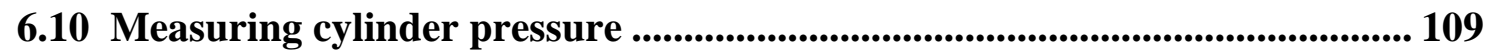

6.11 Conclusions .................................................................................................. 111

$7 \quad$ EXPERIMENTAL RESULTS..................................................................112

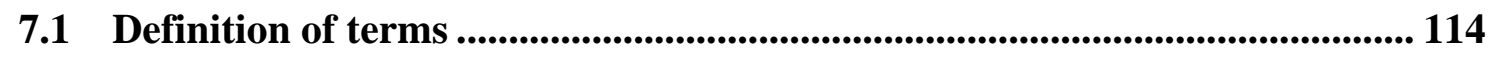


7.2 Effect of the moving mass.................................................................................... 114

7.3 Effect of the stroke length ................................................................................... 116

7.4 Effect of intake air pressure..................................................................................... 117

7.5 Effect of injection timing .............................................................................. 121

7.6 Effect of load........................................................................................................................ 124

7.7 Effect of fuel amount injected........................................................................ 126

7.8 Peak cylinder pressure analysis .............................................................................. 127

7.8.1 Statistical analysis.................................................................................. 127

7.8.2 Peak pressure vs. maximum position............................................................ 131

7.8.3 Pressure-position diagram.............................................................................. 133

7.9 Heat release rate.......................................................................................................... 139

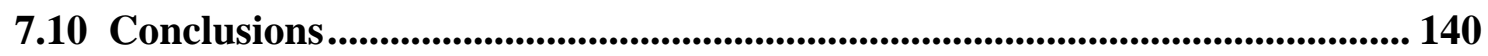

8 COMPARING SIMULATION AND EXPERIMENTAL DATA ......................141

8.1 Effect of moving mass................................................................................................... 141

8.2 The effect of stroke length ........................................................................................ 143

8.3 The effect of the amount of fuel injected ........................................................ 144

8.4 The effect of injection timing ................................................................................ 146

8.5 Effect of load.................................................................................................................. 149

8.6 Results of the predictor/simulator model ........................................................... 150

8.7 Conclusions ..................................................................................................................... 153

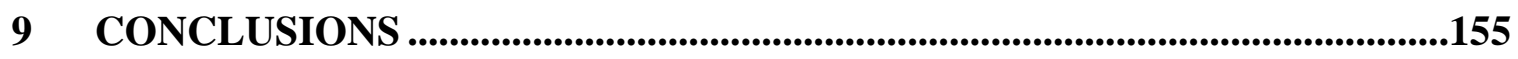

10 RECOMMENDATIONS FOR FUTURE WORK...............................................158

10.1 Lessons learned (positive).......................................................................................... 158

10.2 Lessons learned (negative) ................................................................................... 159

REFERENCES..........................................................................................................161 
APPENDIX A

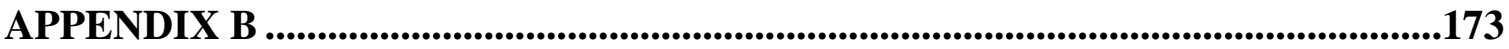

APPENDIX C ............................................................................................................................177

MasterProgram ................................................................................................................... 177

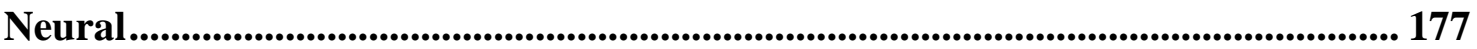

GlobalMainFrame3......................................................................................................... 178

Experiment ............................................................................................................................. 179

Chrom2NNInput ......................................................................................................................... 180

GeneticAlgorithmInitial ........................................................................................................ 181

Inicialize .......................................................................................................................... 182

Simulationnew21WoschniFast........................................................................................... 185

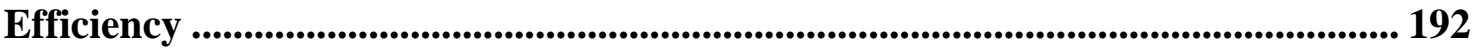

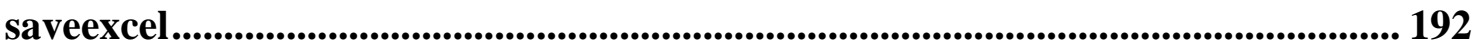

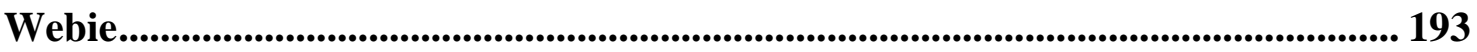

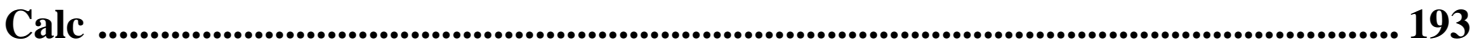

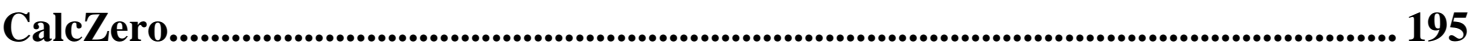

Shift ........................................................................................................................................ 195

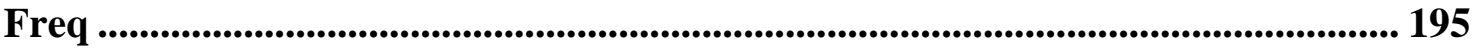

Chrom2NNInputForNeural .............................................................................................. 195 


\section{List of figures}

Figure 1. Hubbert's idealized oil reserve exploration and oil production predictions [1].. 3

Figure 2. The idealized oil discovery and production curves for the world as Hubbert predicted it in 1962 and the modified oil production curve as Ivanhoe predicted it in

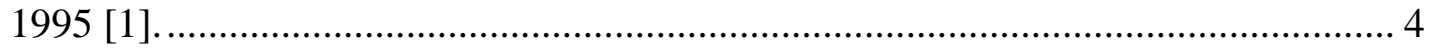

Figure 3. Global annual-mean surface air temperature change derived from the meteorological station network shown for both the annual and 5-year means [5].... 5

Figure 4. Annual and five-year running mean surface air temperature in the contiguous 48 United States relative to the 1951-1980 mean [5].

Figure 5. Carbon dioxide concentration as measured in Mauna Loa, Hawaii. These measurements represent the globally mixed concentration [4] ............................... 7

Figure 6. Greenhouse effect contribution of $\mathrm{CO}_{2}$ and water vapor. $\mathrm{CO}_{2}$ contribution is

only 2\% [7] .......................................................................................... 8

Figure 7. Percent contribution of different sources of man made green house gases to the global green house effect. US CAFE regulated vehicular emissions $1.5 \%$ [7]. ....... 8

Figure 8. NOx emissions standards for passenger cars and light duty trucks for years

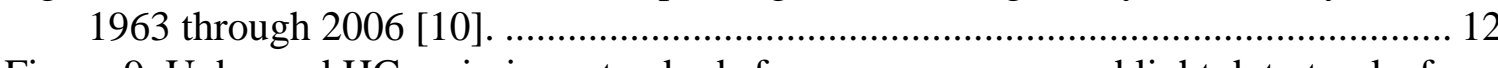

\section{8} 8

Figure 9. Unburned HC emissions standards for passenger cars and light duty trucks for

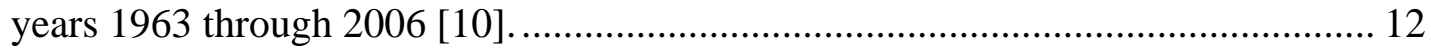

Figure 10. CO emissions standards for passenger cars and light duty trucks for years 1963

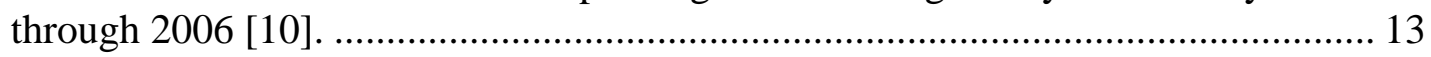

Figure 11. PM (particulate matter) emissions standards for passenger cars and light duty

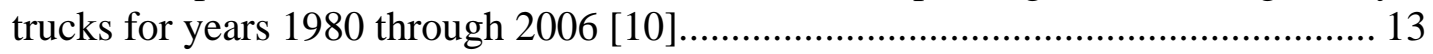

Figure 12. Power flow diagram for a conventional vehicle [17] .................................. 18

Figure 13. Power flow diagram for an electric vehicle [17] ......................................... 19

Figure 14. Power flow diagram for a typical series hybrid electric vehicle [17]............. 21

Figure 15. Power flow diagram for parallel HEV when the electric motor is before the transmission and it assists the internal combustion engine. The electric motor can also be positioned pre- or post-transmission or on a different axle or wheel [17].... 24

Figure 16. Power flow diagram for parallel HEV when the ICE assists the electric motor. Another version of parallel HEVs is when the electric motor is after the transmission. In that case the inefficiency of the transmission does not affect the power of the motor [17].............................................................................. 24

Figure 17. Power flow diagram for the Series-Parallel HEV [17].................................. 26

Figure 18. Power flow diagram for the planetary combination hybrid [17] .................... 28

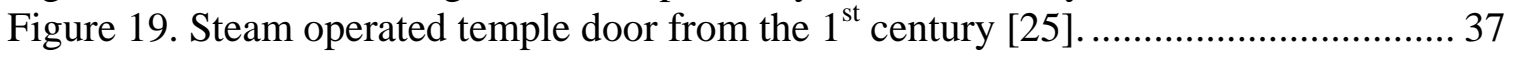

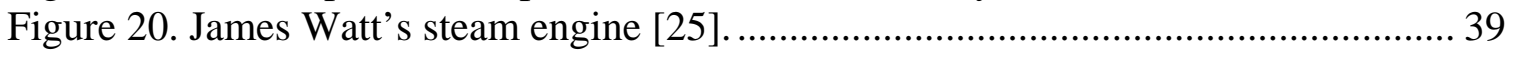

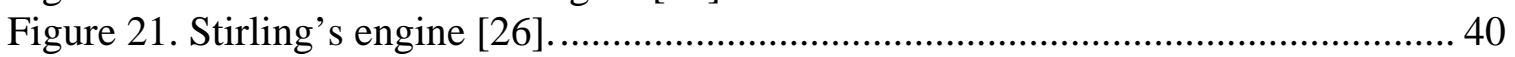

Figure 22. The four stages of operations of the Stirling engine [28] .............................. 41

Figure 23. The four stages of operation of the free-piston Stirling engine [32]............. 43

Figure 24. Pressure-volume diagram for constant-volume, limited-pressure, and constant-

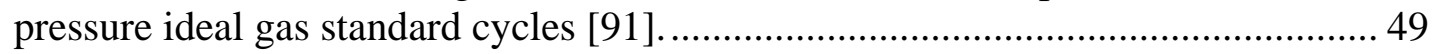

Figure 25. Single piston linear engine configuration............................................... 54

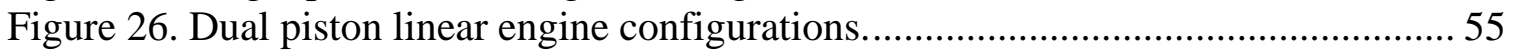

Figure 27. Opposed piston linear engine configuration............................................ 55

Figure 28. Four-cylinder complex configuration.................................................. 56 
Figure 29. Four-cylinder dual piston linear engine configuration................................ 56

Figure 30. Four-cylinder opposed-piston configuration. .......................................... 57

Figure 31. Operating sequence of an opposed piston linear engine gasifier [57]............ 62

Figure 32. The dual piston hydraulic pump developed at Tampere University of

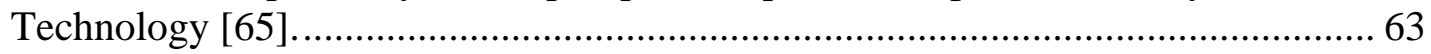

Figure 33. The single piston hydraulic pump developed at Innas B.V. [62]................... 64

Figure 34. The homogeneous-charge compression-ignition linear engine-alternator developed at Sandia National Laboratories [73]................................................... 66

Figure 35. The linear engine developed at West Virginia University, $1^{\text {st }}$ generation [82].

Figure 36. The linear engine can be thought of a mass spring system. The forces acting on the alternating mass (translator) define its motion.......................................... 70

Figure 37. The free body diagram of the linear engine. Gravity is represented by $g$ and

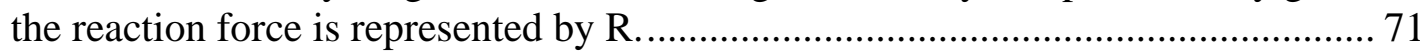

Figure 38. Simulated and experimental translator position after engine was turned off by cutting the fuel. (This phenomena is referred to ringing down).

Figure 39. Simulated vs. experimental translator position after engine was turned off (ringing down). Correlation coefficient: 0.995 .

Figure 40. The flow diagram of the predictor/simulator algorithm used to find the engine with the highest efficiency.

Figure 41. The neural network architecture used for predicting engine efficiency based on bore, stroke, free travel, mass of translator, amount of fuel injected, injection timing, ignition timing. IW and LW indicate the weights in the input and the hidden layer respectively. $b(1)$ and $b(2)$ indicate the thresholds in the input and the hidden layer

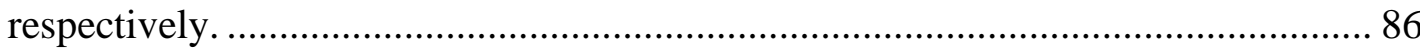

Figure 42. The second-generation linear engine at WVU. ........................................ 92

Figure 43. The linear engine developed at West Virginia University [109].................... 94

Figure 44. Flowchart of the linear engine control. Translator position was a feedback input. Mean, Spark threshold, injection threshold, and fueling rate were user defined

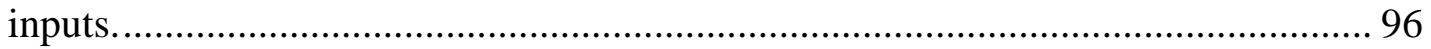

Figure 45. Signal generator signal as input to the controller and the shaped injector signals as response of the controller during controller testing [119]..................... 97

Figure 46. Position of the piston assembly and injection signals vs. time during linear engine testing. The controller provided adequate injection [119]. ..........................99

Figure 47. The control box of the linear engine. The IGBTs are shielded in the left hand side of the box. The panels from left to right are: IGBT drivers, micro-controller, injector drivers, and ignition drivers. The potentiometers in the bottom right corner are mean, injection position, spark position, and fueling rate. 98

Figure 48. Position and cranking signal during cranking of the engine. Engine did not start.

Figure 49. Position and cranking signal during cranking the engine. Engine sustained operation.

Figure 50. The testing dynamometer for the linear engine was a friction brake on the connection rod.

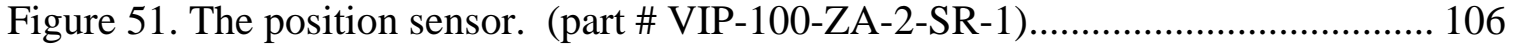

Figure 52. Free body diagram of the translator. 
Figure 53. The maximum and minimum value of translator position.

Figure 54. Unmodified and compensated pressure data for both cylinders. Run \# 14.. 110 Figure 55. The effect of overcharging on output power for different injection timings. Increased charging pressure results in increased power output. (Experimental results)

Figure 56. The effect of overcharging on efficiency for different injection timings. Increased charging pressure resulted in increased efficiency. (Experimental results)

Figure 57. The effect of overcharging on operating frequency for different injection timings. Increased charging pressure resulted in higher operating frequency. (Experimental results).

Figure 58. The effect of overcharging on stalling frequency for different injection timings. Increased charging pressure resulted in higher stalling frequency. (Experimental results).

Figure 59. Operating frequency vs. injection timing. Advanced injection timing slightly increased the no load operating frequency. (Experimental results)

Figure 60. Stalling frequency vs. injection timing. Advanced injection timing resulted in lower stalling frequency. (Experimental results).

Figure 61. Power output vs. injection timing. Advancing the injection timing resulted in higher power output. (Experimental results) .................................................... 122

Figure 62. The effect of injection timing on efficiency. Advanced timing resulted in increased efficiency. (Experimental results)..................................................... 123

Figure 63. Translator position, operating frequency, and load. Increasing load caused the operating frequency to drop. (Experimental results. Run \# 16 in Table 3) ........ 125

Figure 64. Cylinder pressure and load vs. time. The variation in peak cylinder pressure was so high that it made the effect of load unnoticeable. (Experimental results. Run \# 16 in Table 3).... 126

Figure 65. Statistics of the peak pressure analysis. Mean, standard deviation, and coefficient of variant of cylinder pressure with and without spark. Data points between 0 and $8 \mathrm{~mm}$ of injection timing show the left cylinder and data points between 14 and 20 show the right cylinder operation.

Figure 66. Mean, standard deviation, coefficient of variance of peak cylinder pressure vs. injection timing for engine operation without spark. Data are the same as presented in Figure 65.

Figure 67. Mean, standard deviation, coefficient of variance of peak cylinder pressure vs. injection timing for engine operation with the sparking aid on. Data are the same as presented in Figure 65.............................................................................. 130

Figure 68. Peak cylinder pressure and maximum piston position. ............................... 132

Figure 69. Normalized peak cylinder pressure vs. normalized compression ratio. ........ 132

Figure 70. Pressure-position diagram before time alignment. It was for the ring down period.

Figure 71. Pressure-position diagram for the engine ringing down period. Position data were delayed by $0.7 \mathrm{~ms}$. Data are the same as in Figure 70. 134

Figure 72. Pressure-position diagram for Run \# 14 in Table 3. Fueling rate was 3.7 $\mu \mathrm{l} /$ injection. Injection timing was 8 and $20 \mathrm{~mm}$ on the left (blue) and the right (red) side respectively. 
Figure 73. Pressure-position diagram for Run \# 16 in Table 3. Fueling rate was 3.7l $\mu \mathrm{l} /$ injection. Injection timing was 4 and $16 \mathrm{~mm}$ on the left (blue) and the right (red) side respectively. No load.

Figure 74. Pressure-position diagram for Run \# 16 in Table 3. Fueling rate was 3.71 $\mu \mathrm{l} /$ injection. Injection timing was 4 and $16 \mathrm{~mm}$ on the left (blue) and the right (red) side respectively. Load was applied until the engine stalled.

Figure 75. Pressure-position diagram for Run \# 17 in Table 3. Fueling rate was 3.71 $\mu \mathrm{l} /$ injection. Injection timing was 0 and $14 \mathrm{~mm}$ on the left (blue) and the right (red)

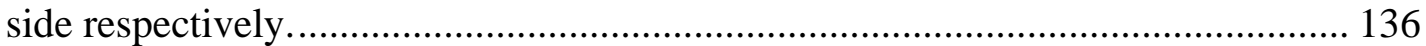

Figure 76. Pressure-position diagram for the simulation results. ................................ 138

Figure 77. A magnified segment of the pressure position diagram in Figure 76............ 138

Figure 78. Heat release rate for simulation (red) and experimental (blue) data. ............ 139

Figure 79. The effect of the moving mass on operating frequency, peak cylinder pressure, and compression ratio. Fuel amount injected was $40 \mu \mathrm{l} /$ injection at $10 \mathrm{~mm}$ before TDC.

Figure 80. The effect of free travel on operating frequency, peak pressure, and compression ratio. Fuel injected was $40 \mu \mathrm{l} /$ injection at $10 \mathrm{~mm}$ before TDC.

Figure 81. Engine operating frequency vs. amount of fuel injected. No load was applied. Heat release was instantaneous.

Figure 82. Lowest operating frequency vs. translator mass. Fuel amount injected was different for each data point.

Figure 83. Heat release rates for different injection timings were calculated by using double Wiebe functions.

Figure 84. Operating frequency, peak pressure, and compression ratio vs. injection timing.

Figure 85. The effect of load on operating frequency. .............................................. 150

Figure 86. predicted and simulated engine efficiency.

Figure 87. Performance of the artificial neural network. The graph shows the mean square error between the desired output and the output predicted by the ANN..... 152 


\section{List of tables}

Table 1. The combination of engine parameters that yielded the highest mechanical efficiency. Bore, effective stroke, total stroke, fuel mass, load force, and injection timing were the input parameters to the predictor/simulator; operating frequency, compression ratio, mechanical efficiency, and output power were the simulated parameters. ........................................................................................................ ii

Table 2. CAFE standards for 1978 to 2003 [11] ............................................................ 10

Table 3. Summary of the test runs on the linear engine.................................................. 113

Table 4. The effect of translator mass, different shaft materials, on the operating frequency. (experimental results)

Table 5. The effect of stroke length on the operating frequency. (experimental results)

Table 6. The effect of overcharging on output power, operating frequency and stalling frequency at different injection timings. (experimental results)............................. 118

Table 7. The effect of fuel amount injected on operating frequency, stalling frequency, output power and efficiency for different injection timing positions. ..................... 127

Table 8. Statistics of the maximum cylinder pressure for different injection timings for the overcharged case............................................................................................. 128

Table 9. The effect of the moving mass on operating frequency, peak cylinder pressure, and compression ratio.

Table 10. The effect of free travel on operating frequency, peak pressure, and compression ratio. Fuel injected was $40 \mu \mathrm{l} /$ injection at $10 \mathrm{~mm}$ before TDC

Table 11. The effect of injection timing on operating frequency, compression ratio, and peak cylinder pressure.

Table 12. The combination of engine parameters that yielded the highest mechanical efficiency 


\section{Nomenclature}

a

a

A

Ah

APU

BDC

CAFE

CC

CI

CNG

$\mathrm{CO}$

$\mathrm{CO}_{2}$

$\mathrm{C}_{\mathrm{p}}$

$\mathrm{C}_{\mathrm{V}}$

CVT

DICI

EPA

EV

$\mathrm{F}_{\mathrm{c}}$

$f_{d}$

$\mathrm{F}_{\mathrm{e}}$

$F_{f}$

FFE

$\mathrm{F}_{\mathrm{i}}$

$\mathrm{F}_{\mathrm{g}}$

$\mathrm{F}_{1}$

$\mathrm{F}_{\mathrm{t}}$

g

$\gamma$

$\mathrm{h}_{\mathrm{i}}$

$\mathrm{H}_{2}$

$\mathrm{H}_{2} \mathrm{O}$

$\mathrm{HC}$

HCCI

HEV

H-HMMWV

HP

HVSim

ICE

$\mathrm{kg}$

$\mathrm{kW}$

$\mathrm{lb}$

LPG

$\mathrm{m}$
Acceleration

Constant

Area

Ampere-hour

Auxiliary power unit

Bottom dead center

Corporate average fuel economy

Cubic centimeter

Compression ignition

Compressed natural gas

Carbon monoxide

Carbon dioxide

Specific heat at constant pressure

Specific heat at constant volume

Continuously variable transmission

Direct injection compression ignition

Environmental Protection Agency

Electric vehicle

Gas force (compression)

Friction coefficient

Gas force (expansion)

Friction force

Fractional factorial experiment

Inertial force

Gas force

Load force

Tension force

Gravity

Ratio of specific heats $\left(c_{p} / c_{v}\right)$

Specific enthalpy

Hydrogen

Water

Hydrocarbons

Homogeneous charge compression ignition

Hybrid electric vehicle

Hybrid electric high mobility multipurpose wheeled vehicle (Humvee)

Horse-power

Hybrid vehicle simulator

Internal combustion engine

Kilogram

Kilowatt

Libra (Pound)

Liquefied petroleum gas

Meter 


\begin{tabular}{|c|c|}
\hline $\mathrm{m}$ & Mass \\
\hline$\mu$ & Friction coefficient \\
\hline $\mathrm{m}_{\mathrm{cr}}$ & Mass flowing into and out of the crevices \\
\hline mpg & Miles per gallon \\
\hline $\mathrm{M}_{\mathrm{d}}$ & Diffusion burn coefficient \\
\hline $\mathrm{M}_{\mathrm{p}}$ & Premix burn coefficient \\
\hline MY & Model year \\
\hline $\mathrm{N}$ & Newton \\
\hline $\mathrm{Ni}-\mathrm{MH}$ & Nickel-metal-hydride \\
\hline NOx & Oxides of nitrogen \\
\hline $\mathrm{p}$ & Cylinder pressure \\
\hline $\mathrm{P}_{\mathrm{g}}$ & Gas pressure \\
\hline PEM & Proton exchange membrane \\
\hline PM & Particulate matter \\
\hline ppm & Parts per million \\
\hline$\frac{d Q}{d t}$ & Heat release rate \\
\hline $\mathrm{Q}_{\mathrm{ch}}$ & Chemical energy released during combustion \\
\hline $\mathrm{Q}_{\mathrm{d}}$ & Heat released in the diffusion burn \\
\hline $\mathrm{Q}_{\mathrm{ht}}$ & Heat transfer to the cylinder walls \\
\hline $\mathrm{Q}_{\mathrm{p}}$ & Heat released in the premix burn \\
\hline $\operatorname{Re}$ & Reynold's number \\
\hline $\mathrm{R}$ & Reaction force \\
\hline RCEM & Rapid compression-expansion machine \\
\hline R \& D & Research and development \\
\hline S & Second \\
\hline SNL & Sandia National Laboratories \\
\hline SOC & State of charge \\
\hline SOFC & Solid oxide fuel cell \\
\hline SI & Spark ignition \\
\hline TDC & Top dead center \\
\hline THS & Toyota Hybrid System \\
\hline t & Actual time \\
\hline $\mathrm{T}$ & Average charge temperature \\
\hline$t_{d}$ & Time that the diffusion burn takes \\
\hline$t_{p}$ & Time that the premix burn takes \\
\hline $\mathrm{T}_{\mathrm{w}}$ & Average wall temperature \\
\hline $\mathrm{U}_{\mathrm{s}}$ & Sensible energy \\
\hline US & United States \\
\hline $\mathrm{V}$ & Volume \\
\hline V & Volt \\
\hline $\mathrm{V}_{\mathrm{c}}$ & Cylinder volume \\
\hline $\mathrm{V}_{\mathrm{p}}$ & Piston velocity \\
\hline $\mathrm{W}$ & Watt \\
\hline $\mathrm{W}$ & The work on the piston \\
\hline WVU & West Virginia University \\
\hline
\end{tabular}




\section{Objectives}

This dissertation intended to show the need for alternative vehicle and fuel research, and to show that hybrid vehicles provide a relief for the environmental problems. Further objective was to show how linear engines fit into this concept as auxiliary power units for hybrid electric vehicles. There were two main objectives that this dissertation was to accomplish. The first one was to develop a working prototype of a two-stroke, two-cylinder, dual piston linear engine and study engine behavior. The second one was to develop a simple simulation model and perform engine simulation. The objective of the simulation was to show that the trends in the experimental data and the simulation data were similar. Further objective was to gain information on the operation regions that were not explored by the experiments. The study was to look at the effect of translator mass, stroke length, amount of fuel injected, fueling timing, and load on engine behavior, namely operational frequency, efficiency, compression ratio, and cylinder pressure. The ultimate goal of the simulation was to define what combination of engine parameters would provide the highest engine efficiency. 


\section{Introduction}

The decreasing crude oil sources of the Earth, the increasing concern about environmental pollution, and fuel consumption and emissions standards force automobile and truck manufacturers into a never-ending effort to design, manufacture, and market less polluting and more fuel efficient vehicles. The intent of this chapter is to give an overview of oil reserve predictions, global environmental issues, and air quality problems in urban areas. The chapter also introduces emissions regulations, CAFE standards, and the Kyoto protocol as an attempted solution for these problems. Based on the facts presented, the incentive and need for alternative vehicle, engine, and fuel research is concluded.

\subsection{Decreasing oil reserves}

In 1962, Dr. M. King Hubbert was studying the oil explorations and production of the continental US and defined his famous curves that showed the historical oil reserve explorations and the predicted oil production [1]. Figure 1 shows Hubbert's idealized oil exploration and prediction curves. 


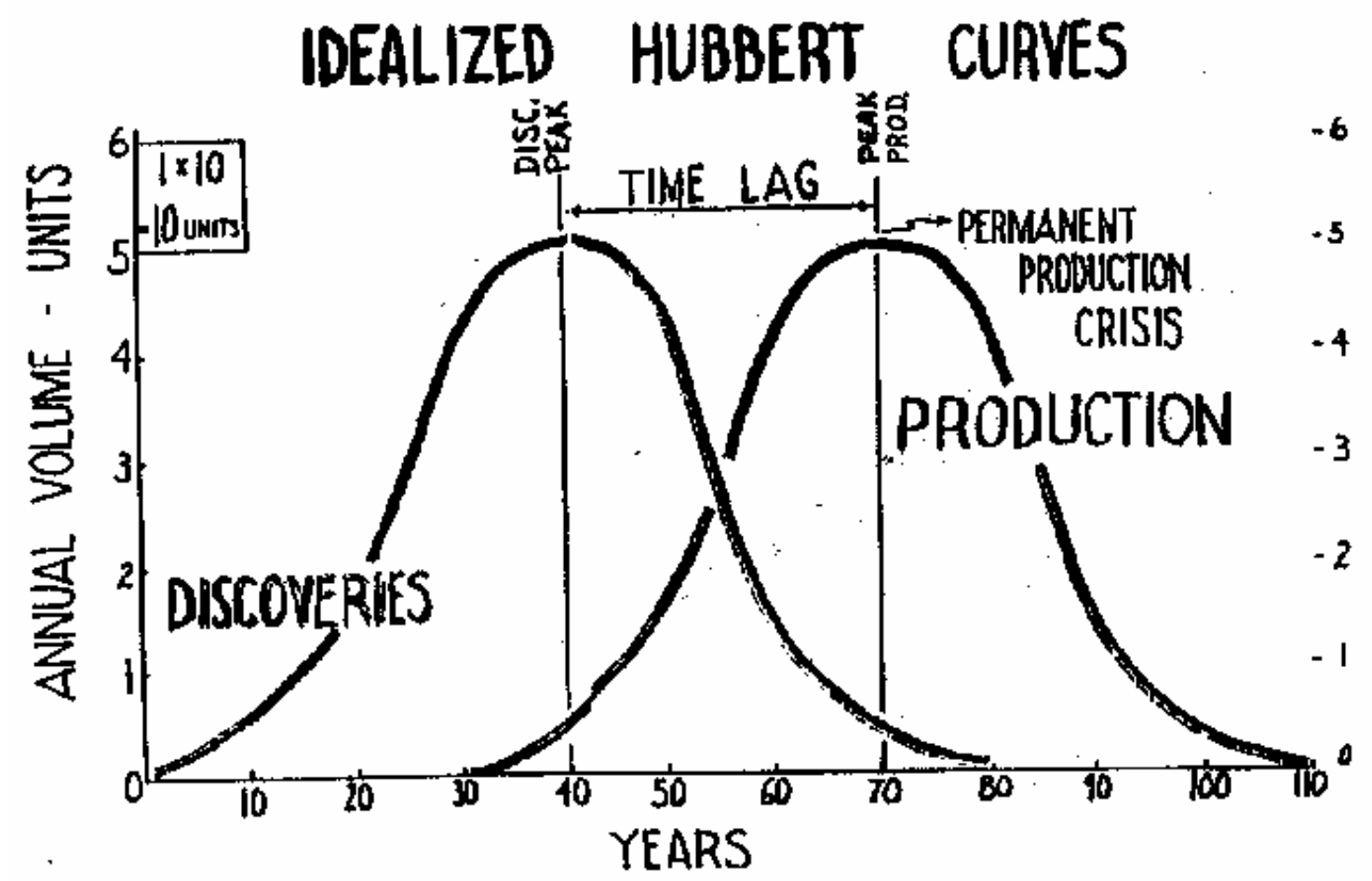

Figure 1. Hubbert's idealized oil reserve exploration and oil production predictions [1].

Although Hubbert made his studies for the 48 continental states of the US, they proved valid for the world's oil reserve explorations as well. Hubbert based his predictions on the fact that the area under the exploration and the production curves must be equal. As the curves show, Hubbert predicted a 100-year period from the beginning of the oil production to the total consumption of the oil reserves. In 1995, Ivanhoe [1] reconsidered the historical oil production data and defined a modified version of Hubbert's prediction for oil production. Figure 2 shows the idealized curves Hubbert predicted and Ivanhoe's modified oil production prediction curve. According to Ivanhoe's predictions the crude oil production will keep at a slight increase until 2010, after that, it will decay and in 2050 the oil production will be at approximately $25 \%$ of that of today. Efforts have been made to utilize other hydrocarbon-based fuels such as compressed natural gas (CNG) and liquefied petroleum gas (LPG) [2] but the final result 
will not be altered: the reserves are going to run out. The decreasing fuel production will result in increasing fuel prices forcing human society to be thrifty with oil and to find alternative energy sources eventually. The energy needs will have to be covered from renewable energy source such as hydraulic and wind power plants and solar energy converters.

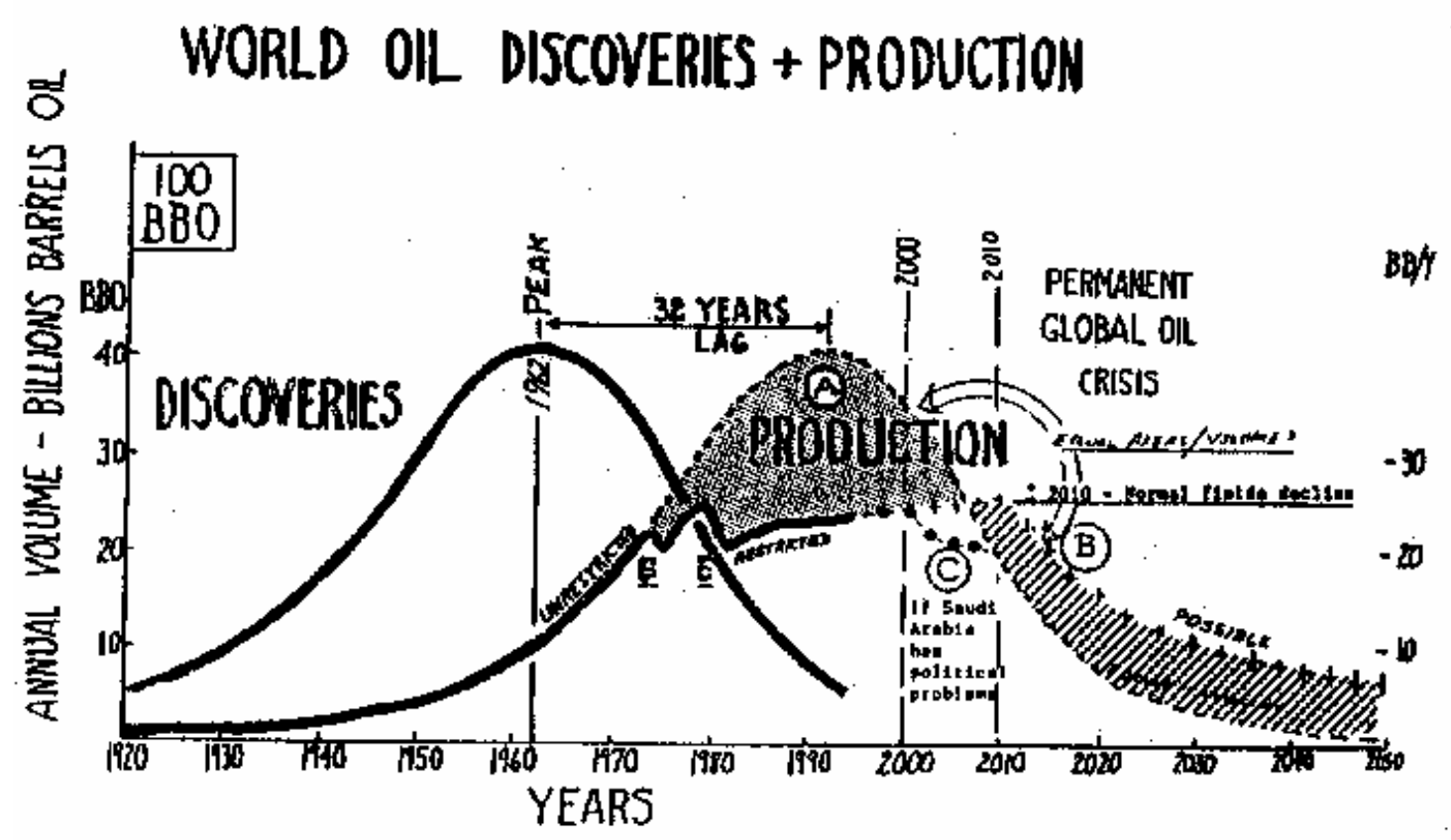

Figure 2. The idealized oil discovery and production curves for the world as Hubbert predicted it in 1962 and the modified oil production curve as Ivanhoe predicted it in 1995 [1].

\subsection{Global warming}

Scientist groups have been concerned about the greenhouse effect, and its result, the global climate change of the Earth. The greenhouse effect is a natural process on Earth that keeps the planet at a temperature that supports the present form of life. Greenhouse gases let the heat of the sun radiate into the atmosphere of Earth to warm the surface and, at the same time, greenhouse gases form an insulating layer and keep a large 
portion of the heat within the atmosphere, just like a greenhouse would do. Although there have been extensive studies conducted on the greenhouse effect, there is no settled agreement on the global warming and its consequences [3-5]. Reference [3] lists a number of sources pro and contra global warming. Figure 3 and Figure 4 show the historical annual-mean surface air temperature data globally and for the US only [5]. The global warming shown in Figure 3 cannot really be seen in Figure 4, which suggests that global warming does not affect the US appreciably. Reference [3] explains this with unreliable sources of data globally. However, error would probably not be consistently increasing year-by-year. This suggests that the global climate change is detectable.

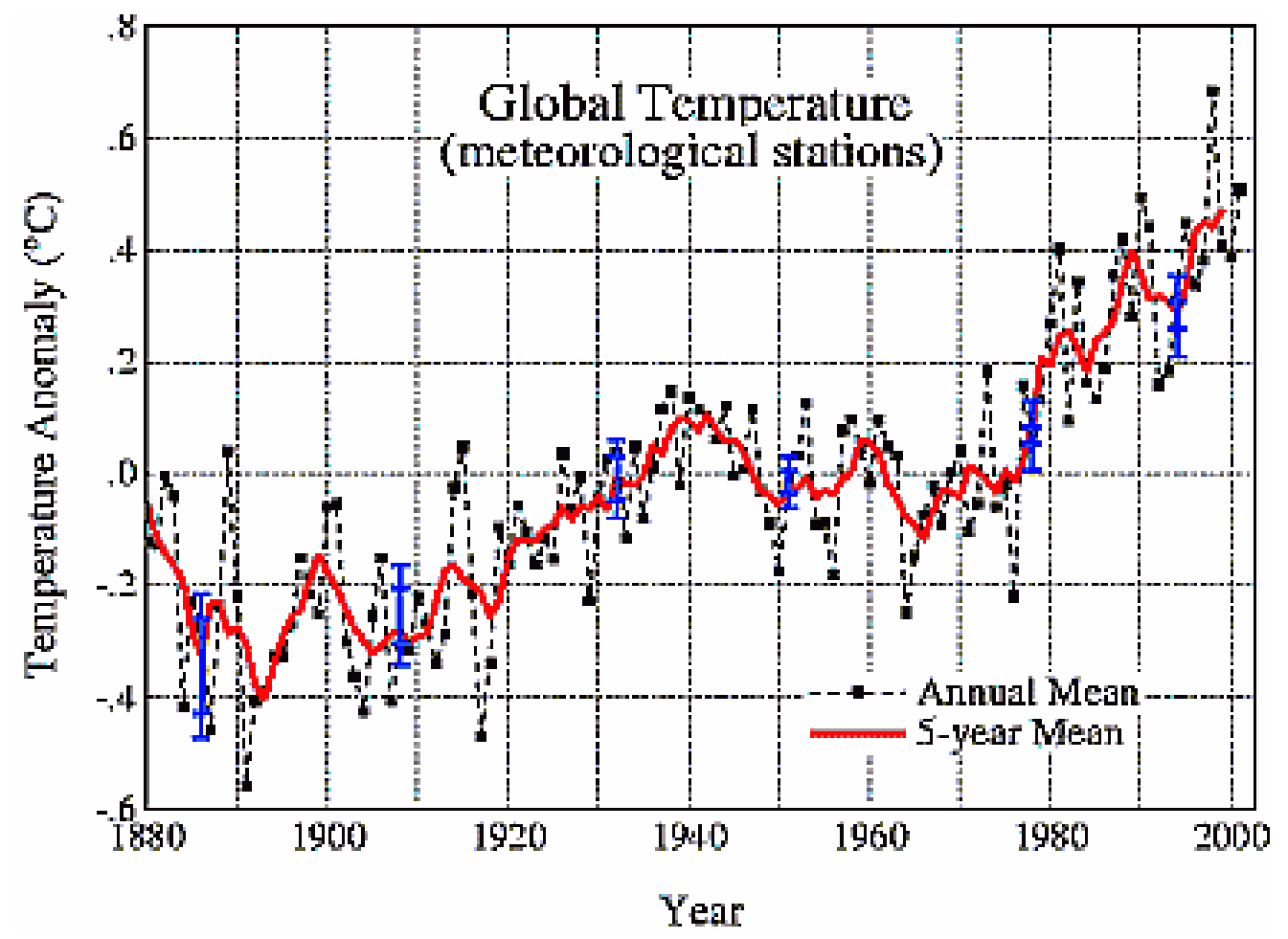

Figure 3. Global annual-mean surface air temperature change derived from the meteorological station network shown for both the annual and 5-year means [5]. 


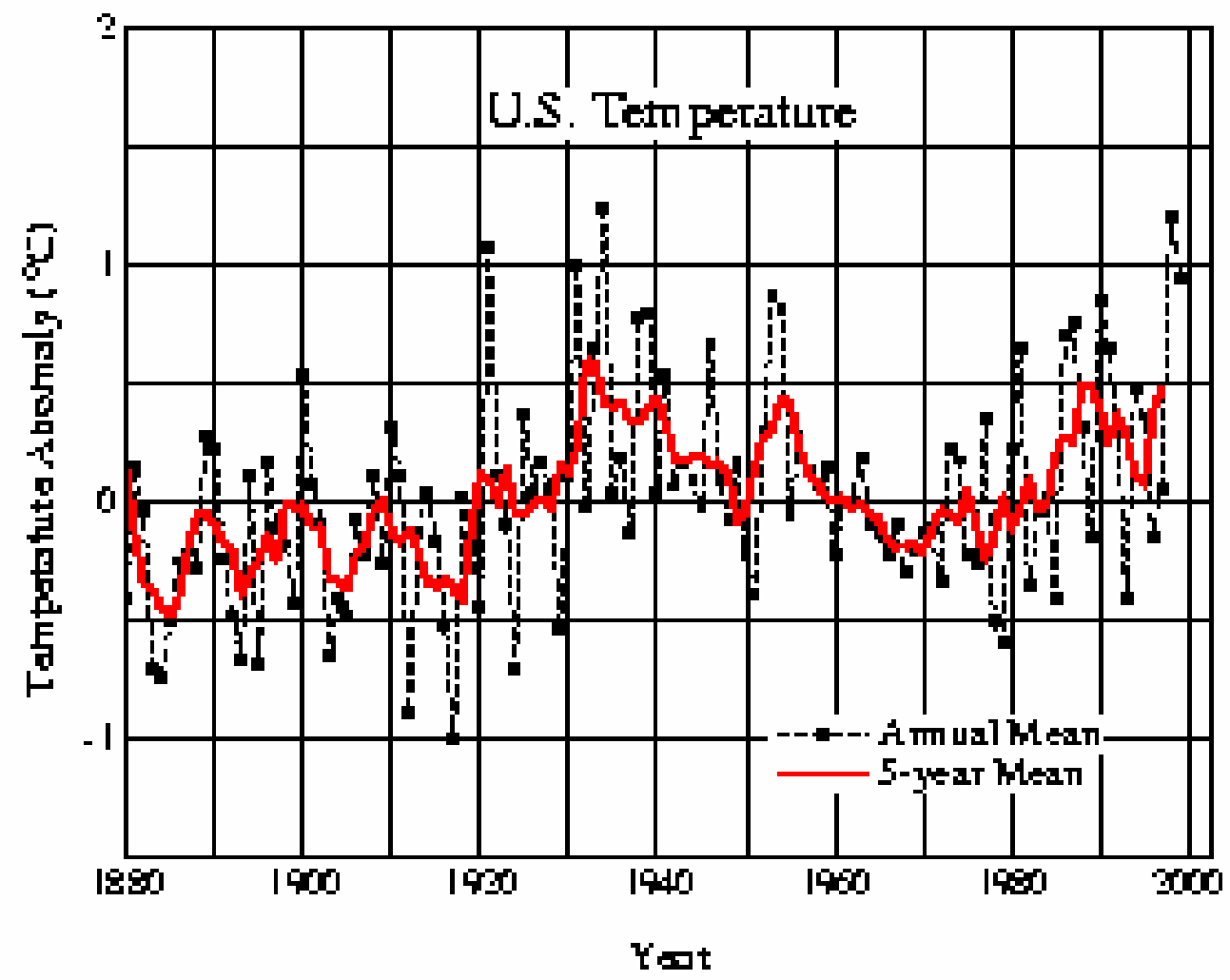

Figure 4. Annual and five-year running mean surface air temperature in the contiguous 48 United States relative to the 1951-1980 mean [5].

Water vapor and carbon dioxide $\left(\mathrm{CO}_{2}\right)$ are the main contributors to the greenhouse effect. Water vapor is natural in the atmosphere of Earth and quickly changing. The amount of water vapor in the air is not influenced by human activity. The amount of $\mathrm{CO}_{2}$ in the atmosphere, on the other hand, can be, and is, influenced by humans. Figure 5 shows the carbon dioxide concentration as measured in Mauna Loa, Hawaii. The measurements represent the globally mixed concentration, as there is minimal local industrial activity in Hawaii [4]. Seasonal fluctuation that can be seen in the graph is due to the natural plant activity. Plants store $\mathrm{CO}_{2}$ during spring and summer then release it due to rotting during fall and winter. 


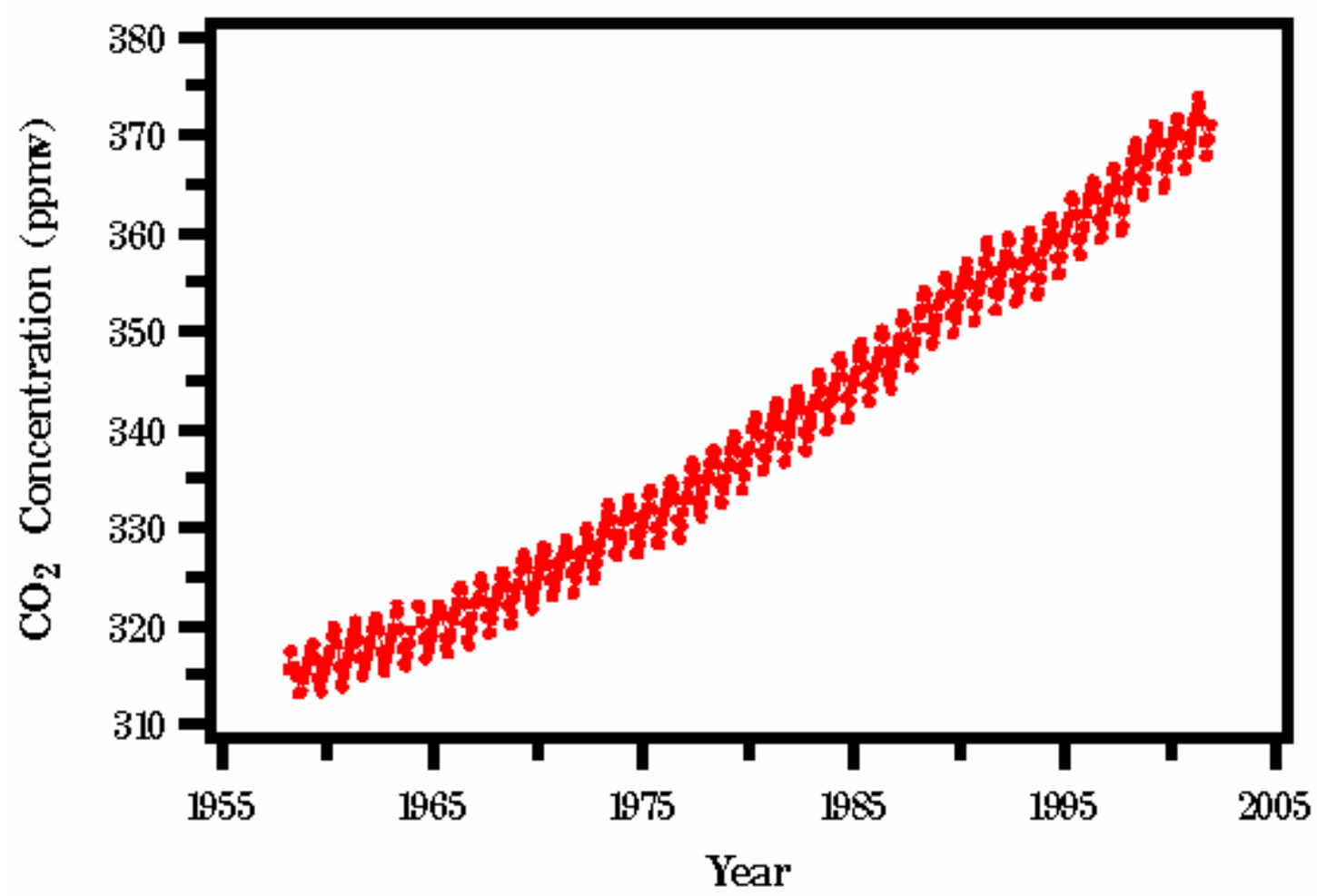

Figure 5. Carbon dioxide concentration as measured in Mauna Loa, Hawaii. These measurements represent the globally mixed concentration [4].

The extension of the graph in Figure 5 suggests that $\mathrm{CO}_{2}$ concentration was stable at $280 \mathrm{ppm}$ [4] before the industrial revolution due to natural sources of $\mathrm{CO}_{2}$ such as volcanic activity, rotting swamps, and plant photosynthesis. The graph itself shows 370 ppm today, which presents a 30\% increase over the last 2 centuries claimed to be due to human activities [4].

Figure 6 shows the greenhouse effect contribution of $\mathrm{CO}_{2}$, which shows that $\mathrm{CO}_{2}$ is responsible in only $2 \%$ of the greenhouse effect. The remaining $98 \%$ is due to water vapor. Although human activity is responsible for only $30 \%$ of the $\mathrm{CO}_{2}$ production, a treaty, the Kyoto Protocol, was established to decrease the $\mathrm{CO}_{2}$ production from human activity [7]. By 1999, 84 countries signed the Kyoto Protocol and agreed to reduce their $\mathrm{CO}_{2}$ production to the level of that in 1990 or lower [8]. 


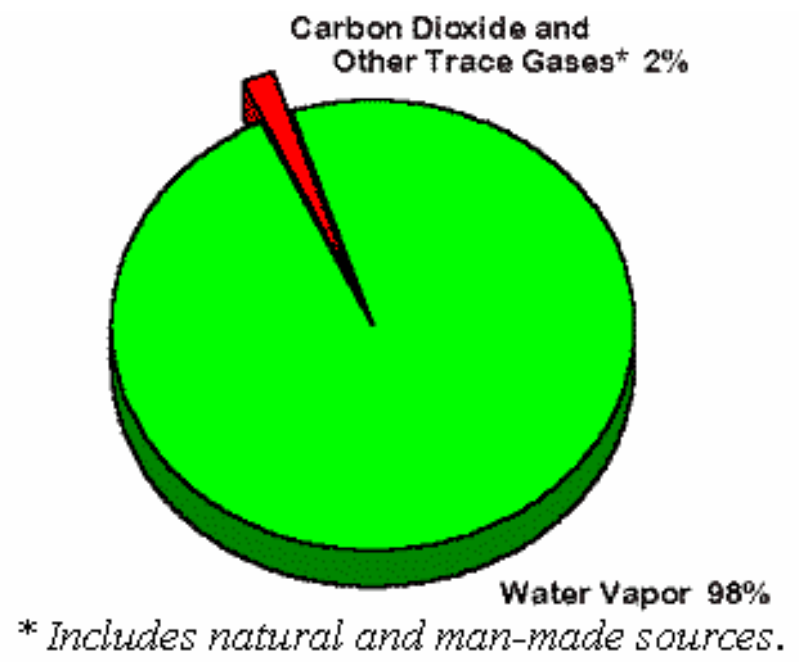

Figure 6. Greenhouse effect contribution of $\mathrm{CO}_{2}$ and water vapor. $\mathrm{CO}_{2}$ contribution is only $2 \%$ [7].

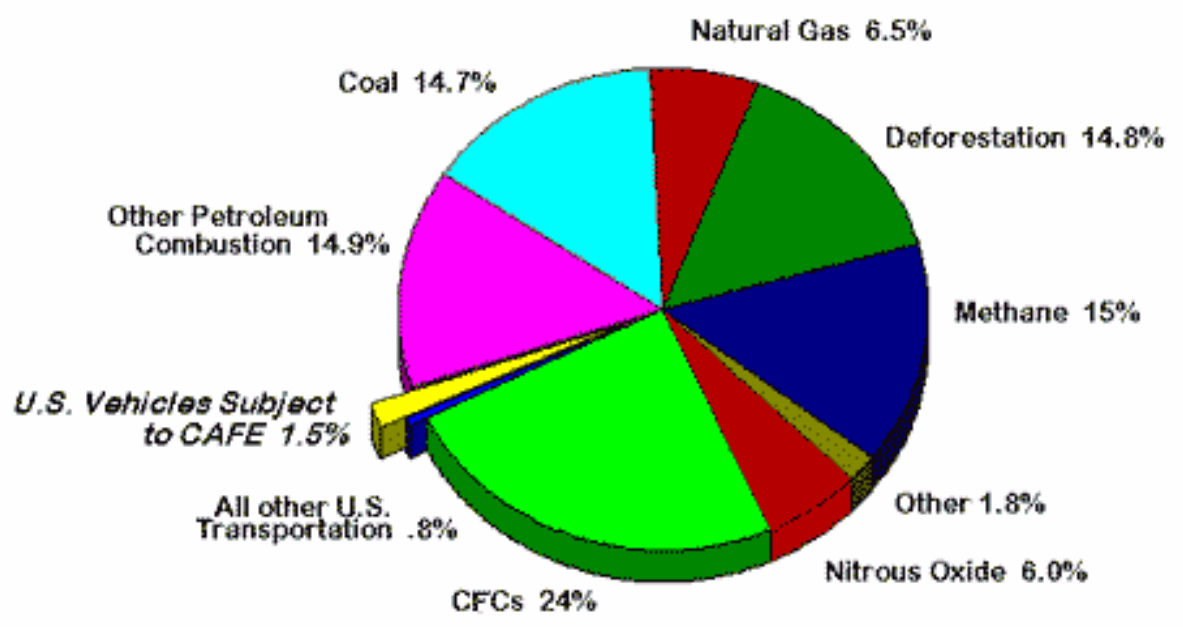

Figure 7. Percent contribution of different sources of man made green house gases to the global green house effect. US CAFE regulated vehicular emissions 1.5\% [7].

Figure 7 shows the contribution of different sources of man made green house gases to the global green house effect. Besides the contribution of other greenhouse gas sources the chart shows the contribution of transportation to the greenhouse effect.

Although $\mathrm{CO}_{2}$ and water vapor are the main contributors, there are some other greenhouse gases that cannot be neglected. Lesser greenhouse gases are methane, 
tropospheric ozone, nitrous oxide, chlorofluorocarbons, particulate matter, carbon monoxide and other reactive gases. They occur in the atmosphere of Earth in trace amounts compared to $\mathrm{CO}_{2}$ and water vapor.

\subsection{Corporate average fuel economy (CAFE) standards}

To decrease total vehicular fuel consumption, the Congress of the United States established the corporate average fuel economy (CAFE) standards that set the standards for passenger car and light duty vehicle fuel economy. $\left(\mathrm{CO}_{2}\right.$ production was not a subject of concern back then.) The present passenger car CAFE standard was set in 1985 at 27.5 mpg and the light duty vehicle CAFE standard was set in 1996 at $20.7 \mathrm{mpg}$. CAFE defines a penalty upon an auto manufacturer whose corporate average fuel economy for the entire fleet sold in the US is lower than the CAFE standard value. The penalty is \$5 per $0.1 \mathrm{mpg}$ for each vehicle sold by that manufacturer. Table 2 shows the CAFE standards for passenger cars and light duty vehicles for 1978 to 2003 [11]. 
Table 2. CAFE standards for 1978 to 2003 [11].

\begin{tabular}{|c|c|c|c|c|}
\hline \multicolumn{5}{|c|}{$\begin{array}{c}\text { Fuel Economy Standards for Passenger Cars and Light Trucks } \\
\text { Model Years (MY) } 1978 \text { through } 2003 \text { (in MPG) }\end{array}$} \\
\hline \multirow{2}{*}{$\begin{array}{l}\text { Model } \\
\text { Year }\end{array}$} & \multirow{2}{*}{$\begin{array}{c}\text { Passenger } \\
\text { Cars }\end{array}$} & \multicolumn{3}{|c|}{ Light Trucks $^{(1)}$} \\
\hline & & $\begin{array}{c}\text { Two-wheel } \\
\text { Drive }\end{array}$ & $\begin{array}{c}\text { Four-wheel } \\
\text { Drive }\end{array}$ & Combined $^{(2),(3)}$ \\
\hline 1978 & $18.0^{(4)}$ & $\ldots$ & $\ldots$ & $\ldots$ \\
\hline 1979 & $19.0^{(4)}$ & 17.2 & 15.8 & $\ldots$ \\
\hline 1980 & $20.0^{(4)}$ & 16.0 & 14.0 & ... $^{(5)}$ \\
\hline 1981 & 22.0 & $16.7^{(6)}$ & 15.0 & $\ldots{ }^{(5)}$ \\
\hline 1982 & 24.0 & 18.0 & 16.0 & 17.5 \\
\hline 1983 & 26.0 & 19.5 & 17.5 & 19.0 \\
\hline 1984 & 27.0 & 20.3 & 18.5 & 20.0 \\
\hline 1985 & $27.5^{(4)}$ & $19.7^{(7)}$ & $18.9^{(7)}$ & $19.5^{(7)}$ \\
\hline 1986 & $26.0^{(8)}$ & 20.5 & 19.5 & 20.0 \\
\hline 1987 & $26.0^{(9)}$ & 21.0 & 19.5 & 20.5 \\
\hline 1988 & $26.0^{(9)}$ & 21.0 & 19.5 & 20.5 \\
\hline 1989 & $26.5^{(10)}$ & 21.5 & 19.0 & 20.5 \\
\hline 1990 & $27.5^{(4)}$ & 20.5 & 19.0 & 20.0 \\
\hline 1991 & $27.5^{(4)}$ & 20.7 & 19.1 & 20.2 \\
\hline 1992 & $27.5^{(4)}$ & $\ldots$ & $\ldots$ & 20.2 \\
\hline 1993 & $27.5^{(4)}$ & $\ldots$ & $\ldots$ & 20.4 \\
\hline 1994 & $27.5^{(4)}$ & $\ldots$ & $\ldots$ & 20.5 \\
\hline 1995 & $27.5^{(4)}$ & $\ldots$ & $\ldots$ & 20.6 \\
\hline 1996 & $27.5^{(4)}$ & $\ldots$ & $\ldots$ & 20.7 \\
\hline 1997 & $27.5^{(4)}$ & $\ldots$ & $\ldots$ & 20.7 \\
\hline 1998 & $27.5^{(4)}$ & $\ldots$ & $\ldots$ & 20.7 \\
\hline 1999 & $27.5^{(4)}$ & $\ldots$ & $\ldots$ & 20.7 \\
\hline 2000 & $27.5^{(4)}$ & $\ldots$ & $\ldots$ & 20.7 \\
\hline 2001 & $27.5^{(4)}$ & $\ldots$ & $\ldots$ & 20.7 \\
\hline 2002 & $27.5^{(4)}$ & $\ldots$ & ... & 20.7 \\
\hline 2003 & $27.5^{(4)}$ & $\ldots$ & $\ldots$ & 20.7 \\
\hline
\end{tabular}

1. $\quad$ Standards for MY (model year) 1979 light trucks were established for vehicles with a gross vehicle weight rating (GVWR) of 6,000 pounds or less. Standards for MY 1980 and beyond are for light trucks with a GVWR of 8,500 pounds or less.

2. For MY 1979, light truck manufacturers could comply separately with standards for four-wheel drive, general utility vehicles and all other light trucks, or combine their trucks into a single fleet and comply with the standard of $17.2 \mathrm{mpg}$.

3. For MYs 1982-1991, manufacturers could comply with the two-wheel and four-wheel drive standards or could combine all light trucks and comply with the combined standard.

4. Established by Congress in Title V of the Motor Vehicle Information and Cost Savings Act.

5. A manufacturer whose light truck fleet was powered exclusively by basic engines which were not also used in passenger cars could meet standards of 14 mpg and 14.5 mpg in MYs 1980 and 1981, respectively.

Revised in June 1979 from 18.0 mpg.

Revised in October 1984 from $21.6 \mathrm{mpg}$ for two-wheel drive, $19.0 \mathrm{mpg}$ for four-wheel drive, and $21.0 \mathrm{mpg}$ for combined.

Revised in October 1985 from 27.5 mpg.

Revised in October 1986 from 27.5 mpg.

Revised in September 1988 from 27.5 mpg. 
In summary, the pie chart in Figure 6 represents the greenhouse effect contribution of $\mathrm{CO}_{2}$, which shows that $\mathrm{CO}_{2}$ is responsible for the greenhouse effect in only 2\%. From Figure 5, it can be concluded that human activity is responsible for 30\% of the $\mathrm{CO}_{2}$ present in the atmosphere. Figure 7 shows that those vehicles that are subjected to CAFE in the US produce only $1.5 \%$ of the $\mathrm{CO}_{2}$ that is made by man. Putting the pieces together yields: $2 \%$ of the greenhouse effect is due to $\mathrm{CO}_{2} ; 30 \%$ of that is due to human activity; $1.5 \%$ of that is emitted by vehicles regulated by the CAFE standard in the US. It can be concluded that the whole passenger car and light duty vehicle fleet in the US contributes to the greenhouse effect only in $0.009 \%$. This number, however, neglects the greenhouse effect of water vapor generated during combustion.

\subsection{EPA emissions standards}

The poor air quality in urban areas caused by vehicular emissions, on the other hand, is of concern and affects the whole local population. The smog lingering around major cities represents a serious health hazard to dwellers of metropolitan areas as being a major contributor to asthma, allergies, and other respiratory diseases [9].

To improve air quality in urban areas and, along with this, decrease health hazards, and improve visibility problems originating from vehicular emissions, the Environmental Protection Agency (EPA) established emissions standards that force vehicle manufacturers to produce vehicles that emit less exhaust emissions year by year. The Clean Air Act of 1963 and its amendments set the emissions standards for both light and heavy-duty vehicles sold in the US [10]. The presently regulated emissions are oxides of nitrogen (NOx), carbon monoxide (CO), unburned hydrocarbons (HC), and particulate matter (PM). Figure 8 through Figure 11 show the emission regulations for 
the four regulated exhaust emissions for passenger cars and light duty trucks for years 1963 through 2006.

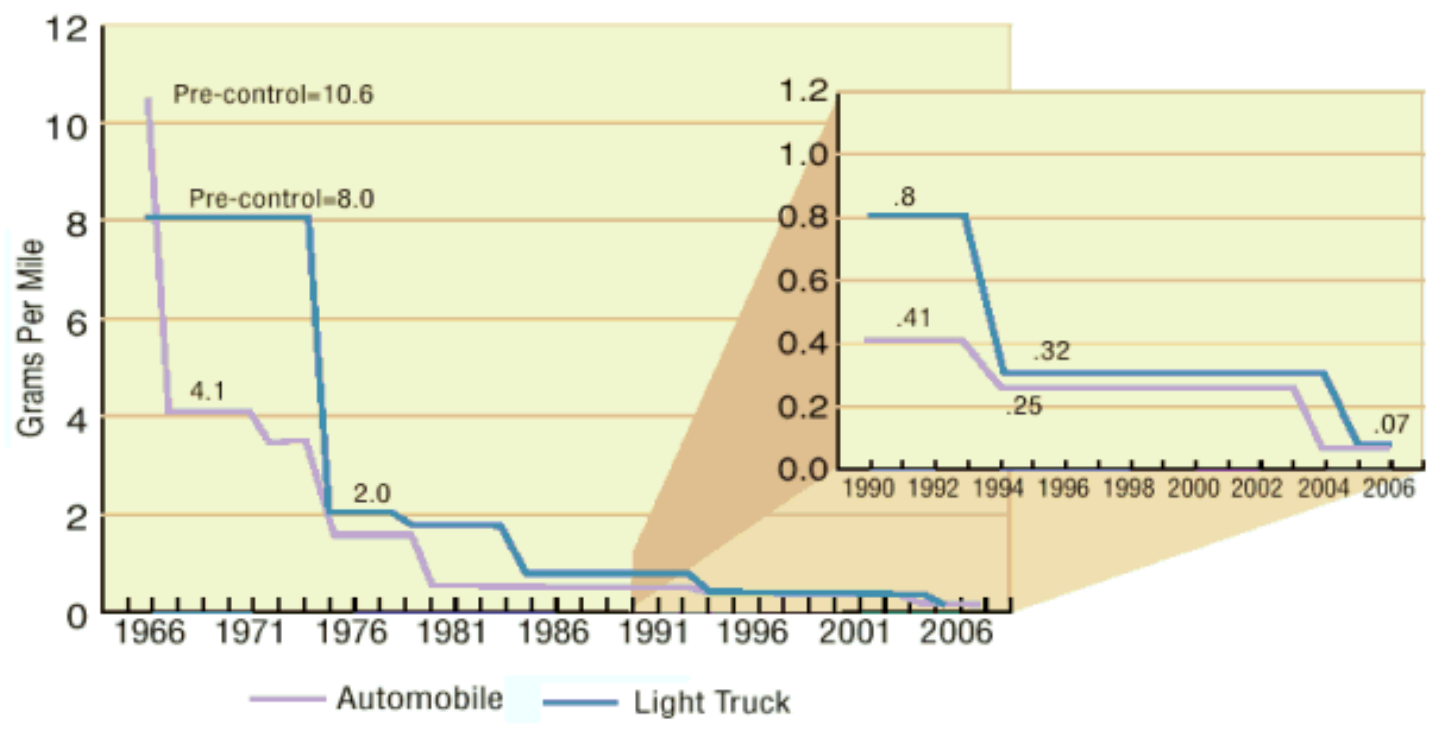

Figure 8. NOx emissions standards for passenger cars and light duty trucks for years 1963 through 2006 [10].

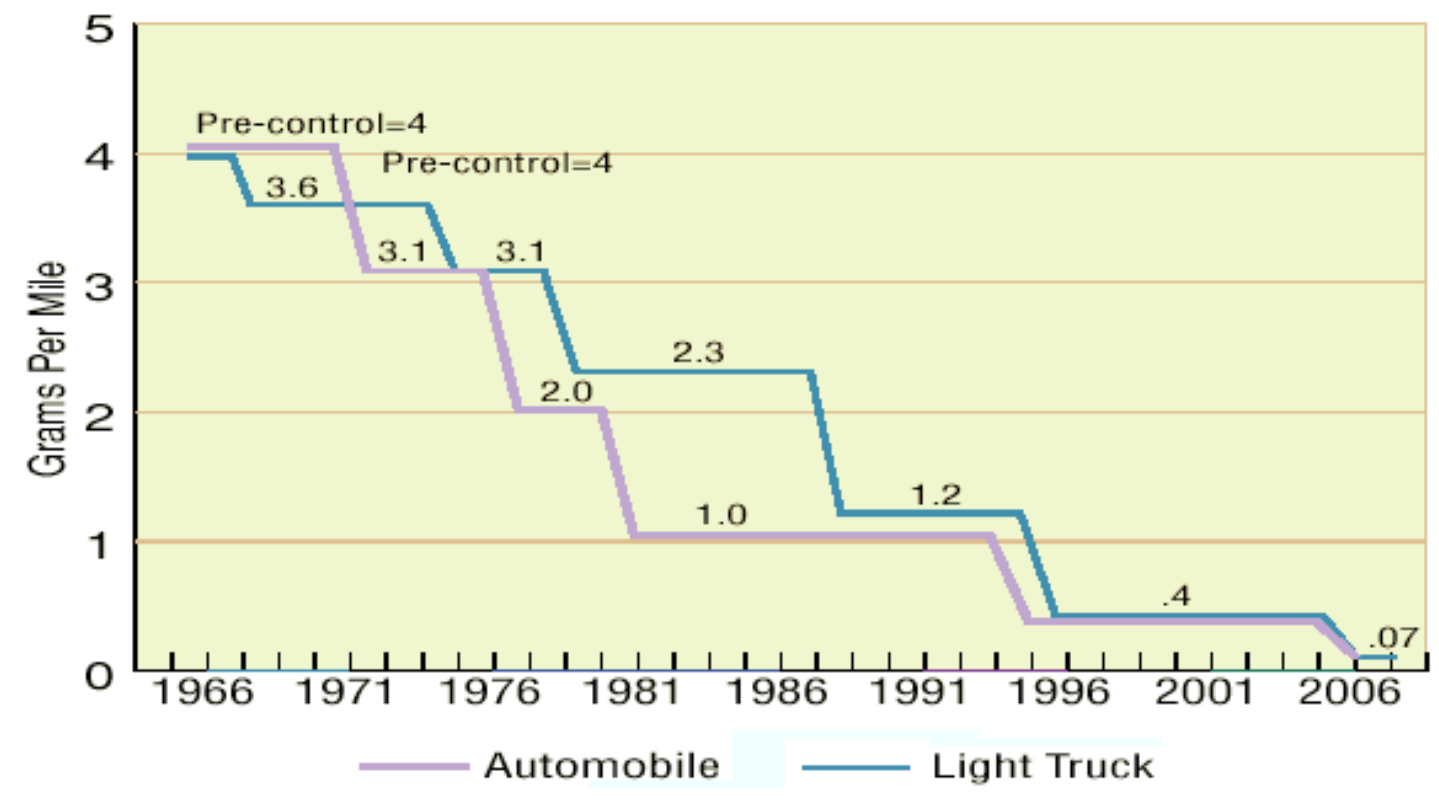

Figure 9. Unburned HC emissions standards for passenger cars and light duty trucks for years 1963 through 2006 [10]. 


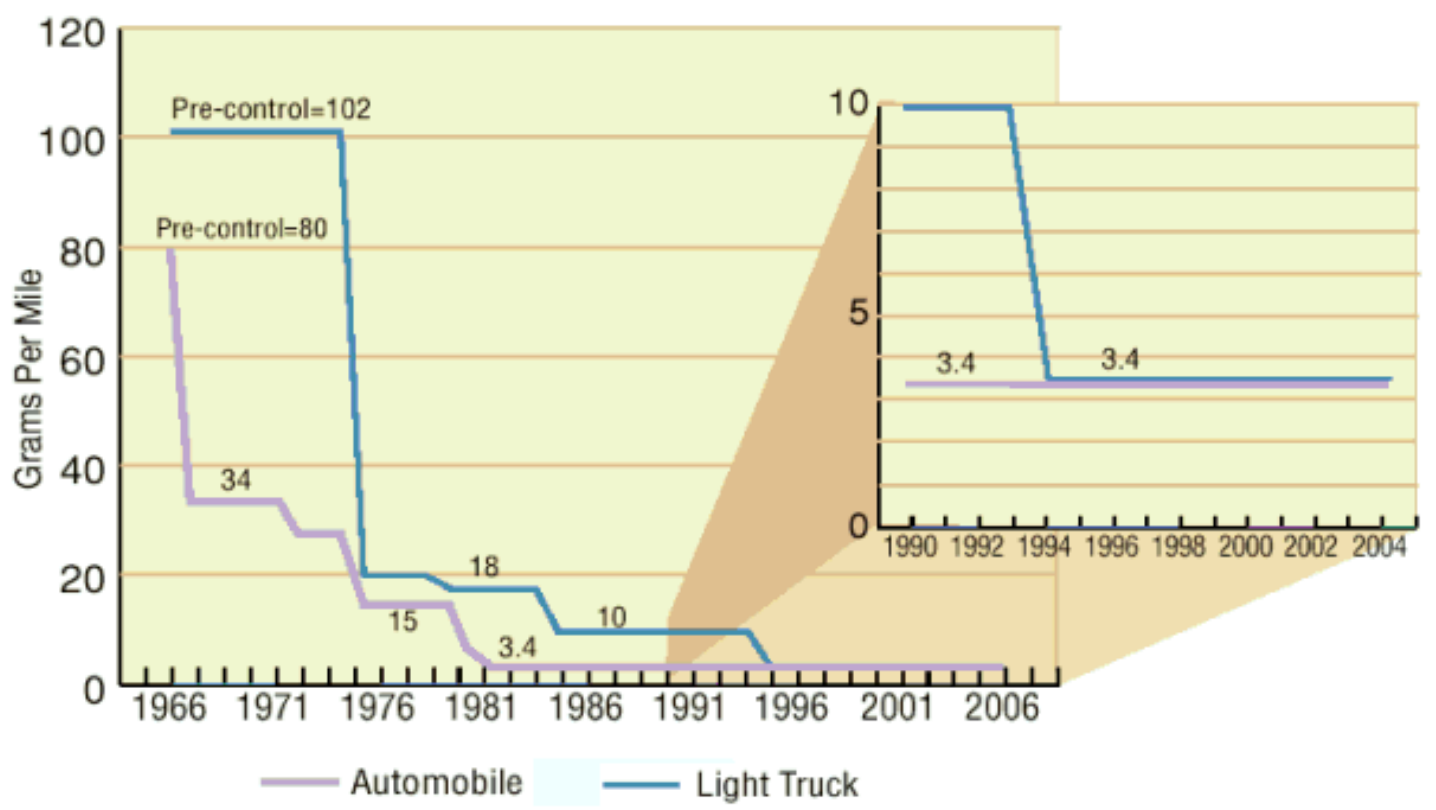

Figure 10. CO emissions standards for passenger cars and light duty trucks for years 1963 through 2006 [10].

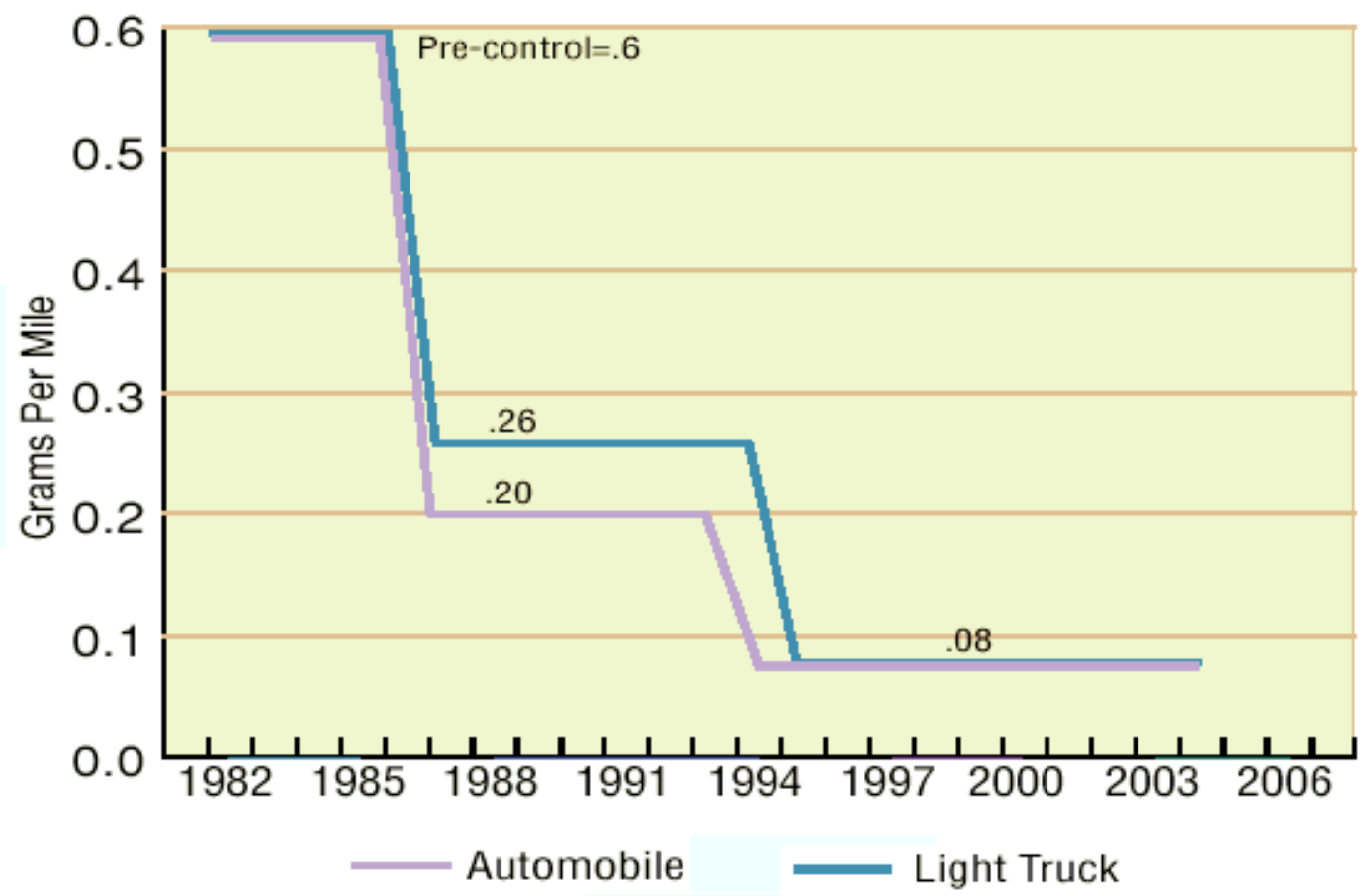

Figure 11. PM (particulate matter) emissions standards for passenger cars and light duty trucks for years 1980 through 2006 [10]. 
Figure 8 through Figure 11 show the tightening tendency in exhaust gas regulation. This trend is the same for the Japanese and European emissions regulations too [9]. In the limit the regulations will reach the level of zero exhaust emissions. However, even electric vehicles can only be considered as zero emission vehicles if they use electrical energy from non-emitting energy sources.

\subsection{Conclusions}

This chapter presented the problem of global climate change and air quality in urban areas. Although the global warming and the results of the greenhouse effect are yet to be determined, the poor air quality in urban areas and the projected oil crisis represent a concern that gives the incentive to the research on alternative fuels and alternative vehicles. 


\section{Hybrid electric vehicles}

Without question, zero emission vehicles are the ultimate goal and solution for emission problems. However, the technology, the infrastructure, and the real need for ZEVs are not developed yet. Hybrid electric vehicles (HEVs) have emerged in the 1990's to fill the gap between today's vehicles and zero emission vehicles. The fact that every major vehicle manufacturer is working on different hybrid designs shows that HEVs have a place on the vehicle market attracting customers and showing potential profit. This chapter presents some history about hybrid electric vehicles and discusses how and why they were invented and their role today. The basic configurations, series and parallel hybrids are introduced, along with the combination hybrid, the planetary hybrid, and the fuel cell hybrid. The chapter also shows some of the major manufacturers’ hybrid designs concluding a trend in hybrid configuration.

\subsection{A little history}

The hybrid electric vehicle concept is not new. The history goes back to 1790 when Nathan Read made the first drawings of a steam carriage and fifteen years later a self-propelled car was invented [14]. After the first attempts to make self-powered vehicles, which were driven by steam engines, two different power sources became usual. One of them was the electric motor, and the other was the internal combustion engine.

The first electric vehicle was made by Professor Stratingh in the Dutch town of Groningen in 1835. Although several electric vehicle manufacturers were established in Europe as well as in America before internal combustion engines became available the electric vehicle did not become a viable option until the Frenchmen, Gaston Plante and 
Camille Faure invented (1865) and improved (1881) the storage battery. On the turn of the century (1899) Baker Electric, in the USA, manufactured an electric vehicle that was reputedly easy to drive, and could cruise a distance of 80 kilometers when fully charged. Reference [12] states that the vehicle was capable of reaching a top speed of $40 \mathrm{~km} / \mathrm{h}$. A 1 hp DC motor powered it. Its operating voltage was $20 \mathrm{~V}$. The vehicle had rechargeable batteries as an energy storage device [12].

Various countries claim to be the first to produce a gasoline-powered automobile. Although there is room for argument, Germany's Karl Benz is now accorded this distinction with the three-wheeler he produced in 1886. The engine, placed over the rear axle, was a horizontal, four-cycle, single-cylinder type with 984cc-volume displacement. The engine was capable of providing $0.9 \mathrm{hp}$ at $400 \mathrm{rpm}$ and could propel the vehicle as fast as $15 \mathrm{mph}$. It was the first automobile equipped with a differential gear [12].

Electric cars were more expensive than gasoline vehicles while electric vehicles were considered more reliable, safer and more convenient. Electric vehicles were also quieter and they did not need cranking unlike internal combustion driven cars, therefore it is no surprise that they were the preferred choice of consumers. However, the electrical circuits were sensitive to humidity and batteries were sensitive to temperature. Between 1890 and 1910, many hybrid electric cars were built. The purpose of hybrids was to improve the handicaps of the single propulsion systems. They were a transition between electric and gasoline cars.

With the development of the starter motor for gasoline cars, the application of the carburetor and liquid fuel, and increased range of the vehicles, the public interest turned from electric to gasoline and electric vehicles and hybrids were forgotten for a long 
while. Although adventurous engineers never stopped designing electric vehicles, the lack of advanced batteries, lack of efficient control and the cheap price of gasoline pushed electric and hybrid electric vehicles into the background until recent years. Nowadays, decreasing crude oil resources and increasing environmental concerns have revitalized the concept of electric vehicles. Hybrids have already returned as the first stage of the change. They fulfill the same role now as they did 100 years ago. Hybrids were a transition from electric to internal combustion engine (ICE) driven vehicles at the turn of the last century, now they are a transition from ICE-driven to electric vehicles.

\subsection{Hybrid configurations}

Several hybrid configurations have been developed over the last 100 years. Although the number of theoretical configurations is limitless, there are some practical limitations such as cost, complexity, and weight that pose as practical boundaries around HEV design. Hybrid vehicles are based on conventional ICE driven vehicle and electric vehicle components. The two basic concepts are series hybrid and parallel hybrid. From the basic configuration, countless combinations can be developed with various configurations and component sizes.

\subsubsection{Advantages and disadvantages of conventional vehicles}

Although everyone is familiar with conventional vehicles, their features are summarized here to form a basis for comparison. Figure 12 shows the layout of a conventional vehicle. 


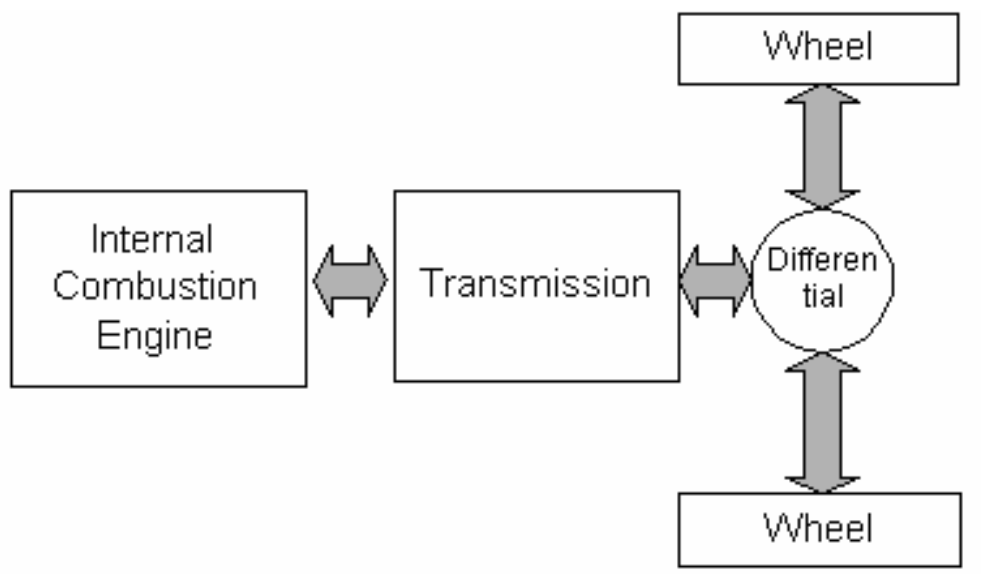

Mechanical Energy Flow

Figure 12. Power flow diagram for a conventional vehicle [17].

In a conventional vehicle the energy source is hydrocarbon-based fuel. An internal combustion engine forms the power converter and drives the wheels via a transmission and a differential. The engine can be spark or compression ignited and it can use a variety of fuels. The transmission can be manual, automatic or a CVT (continuously variable transmission). A conventional vehicle is relatively cheap and easy to control. It does not require extra control besides the engine control unit and the automatic transmission control unit if an automatic transmission is applied. Conventional vehicles are inexpensive, reliable and they have a highly developed refueling infrastructure. As a disadvantage, they use oil-based fuel and emit exhaust emissions.

\subsubsection{Advantages and disadvantages of electric vehicles}

In an electric vehicle the energy source is electricity. An energy storage device stores the electrical energy on board. Although the energy storage device could be a flywheel, a hydraulic accumulator, or an ultra-capacitor, in today's electric vehicles it is 
usually a battery pack. An electric motor converts the electrical energy to mechanical energy and drives the wheels via a differential. The configuration may or may not include a transmission. Figure 13 shows the layout of a typical electric vehicle.

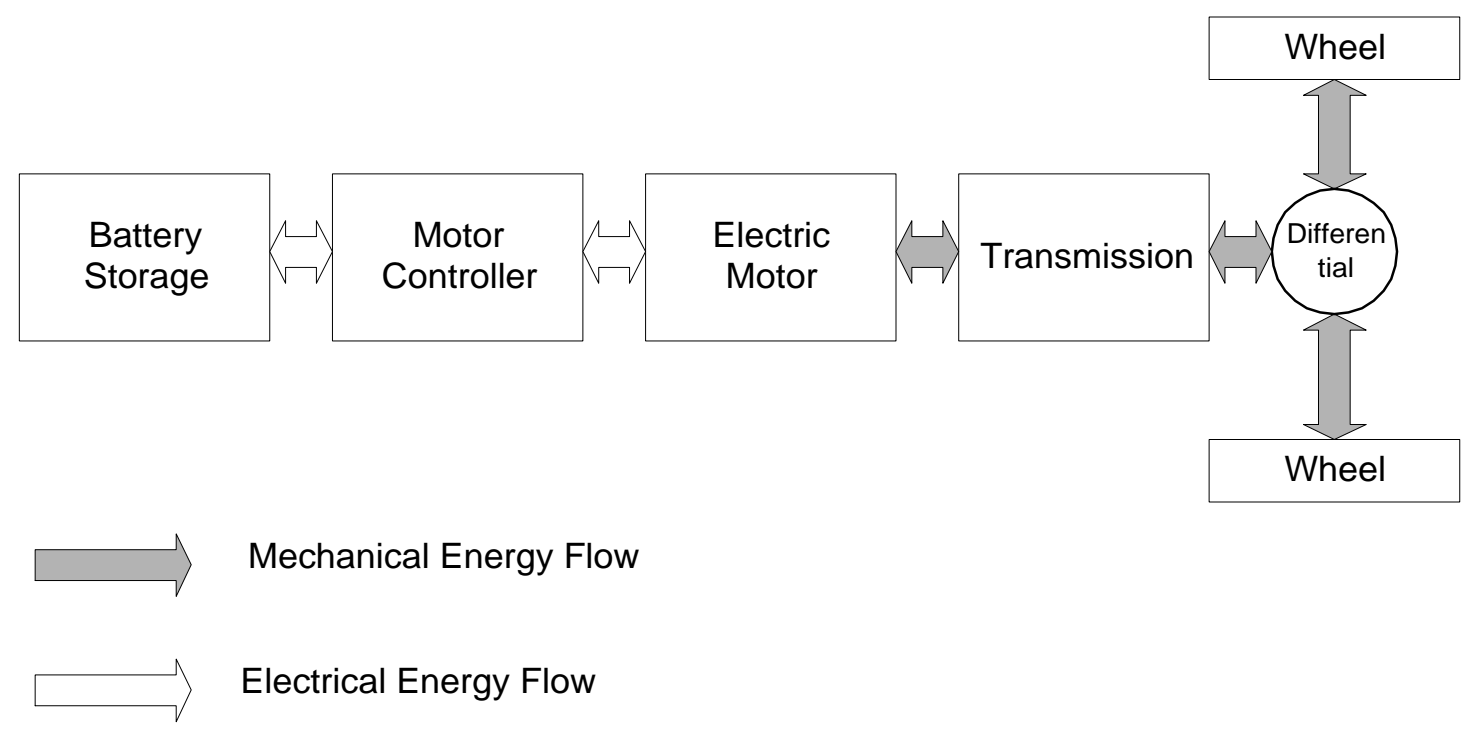

Figure 13. Power flow diagram for an electric vehicle [17].

The main advantage of electric vehicles is that they don't emit exhaust gases at the stage of operation. Although, they are called zero emission vehicles (ZEVs), the electrical energy production is not free of emissions nowadays. According to the "wells to wheels" concept the emissions of the vehicle must be increased by the emissions of any kind related to the vehicle or its fuel such as production and transportation. In that sense EVs, today, are not truly zero emission vehicles because they are charged from the electrical grid and majority of the electricity available from the grid is produced by burning fossil fuels [18].

Another advantage of EVs is their noiseless operation. The application of EVs would decrease the noise level in cities significantly. EVs are also capable of 
regenerative braking. They can capture the kinetic energy that is dissipated on the brake discs in conventional vehicles, and store it in the batteries for later use.

EVs, if they were in mass production, would be competitive with conventional vehicles in complexity and price and even less complicated to control. The disadvantage of the electric vehicle is its short range that is limited by the capacity of the battery pack. Present battery technology provides approximately 100 miles on a single charge depending on vehicle size, battery size and capacity and driving conditions [17]. For example GM claimed that the EV1 had a range 160 miles [19].

The short range of electric vehicles is not the main problem though. While conventional vehicles can be refilled in the matter of a couple of minutes, batteries of EVs may need several hours of charging once they are discharged.

\subsubsection{Advantages and disadvantages of series HEVs}

Series HEVs have a dual energy source: fuel and electricity. An electric motor is coupled either straight to the differential through a gear or chain drive or coupled thorough a gearbox to drive the wheels, just like in an EV. As a range extender, an internal combustion engine (ICE) is coupled to an alternator to supply electrical energy to the electric motor or to charge the batteries. Figure 14 displays the typical layout of a series hybrid electric vehicle. 

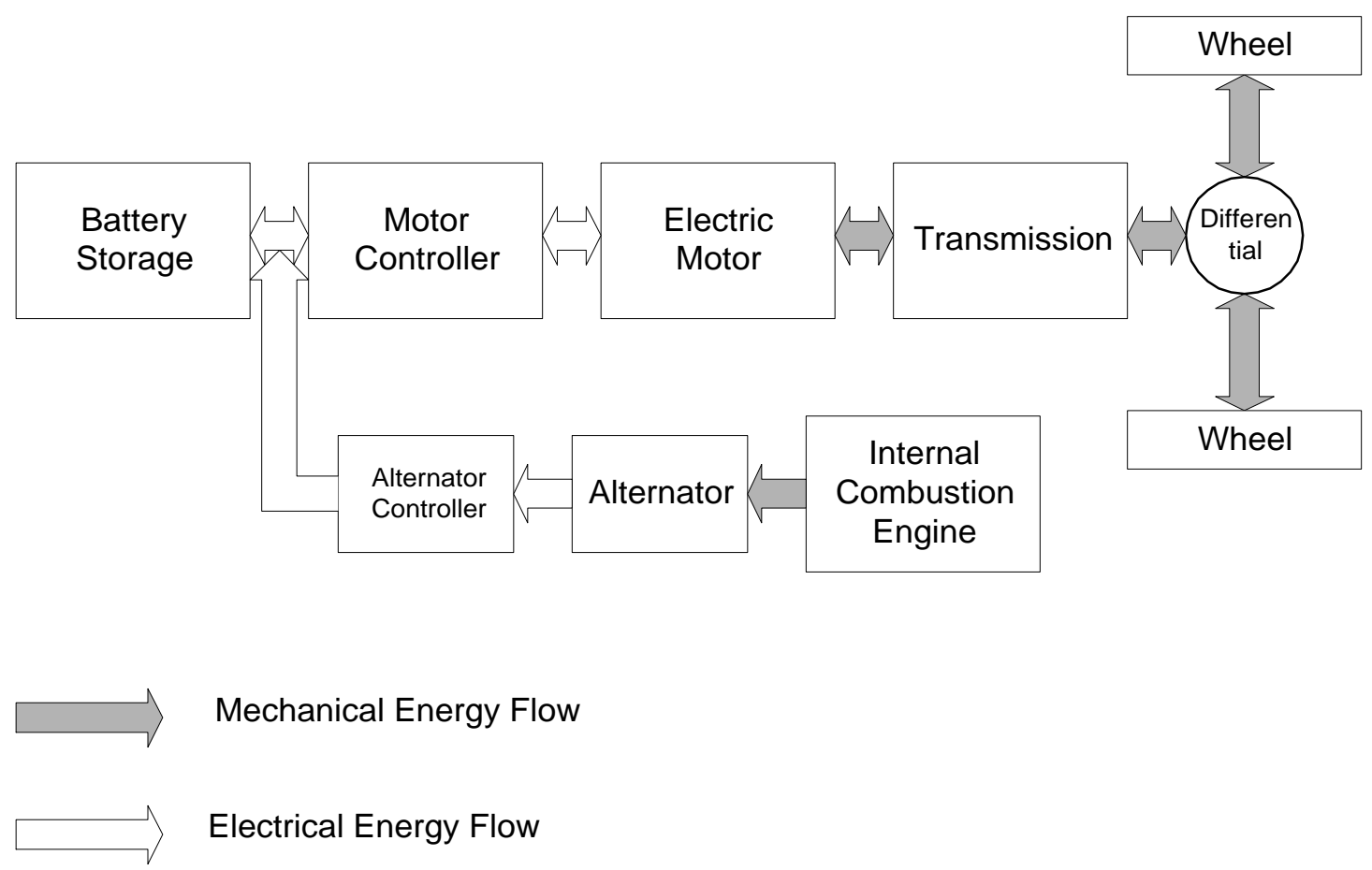

Figure 14. Power flow diagram for a typical series hybrid electric vehicle [17].

In series HEVs there is no physical coupling between the engine and the transaxle. This can reduce the transient operation of the ICE that is especially helpful from an emissions standpoint allowing optimal fueling and ignition control. Under high power demand, often, an engine will fuel heavily to prevent a misfire situation due to an instantaneously high air to fuel ratio. The drawback to a series hybrid electric vehicle is the associated mechanical to electrical to mechanical energy conversion losses. However, this makes it possible for the engine to operate in its most efficient region. The fact that the engine can operate in its most efficient region compensates the energy conversion losses and results in fuel economy improvement that is significant in the city and moderate on the highway. When batteries are full and power demand is low, the engine can be turned off. The design also offers regenerative braking to capture the braking energy and store it in the battery instead of wasting it on the brake disks in the form of heat. 
Series hybrids can be divided into two main groups: load-leveling and loadfollowing series vehicles. In a load-leveling hybrid the auxiliary power unit (APU, engine-alternator assembly) is sized such that it would meet the average power requirement of the vehicle's duty cycle and would operate at its most efficient point ensuring absolutely steady state operation. The battery acts as an energy buffer and supplies the energy needed over the engine's capability when the vehicle requires more, or stores the excess energy when the vehicle requires less energy than what the engine supplies operating at its most efficient point. Although the engine operates at its most efficient point, excessive battery usage can result in high overall inefficiencies due to the fact that batteries can be very inefficient when charged or discharged at high rates. While in load-leveling series hybrids the engine operates at steady state, at its most efficient point, in a load-following hybrid the engine power output more or less follows the power demand of the electric motor resulting in decreased engine efficiency. A diesel-electric railway engine would be a 100\% load-following hybrid as it does not have an energy storage device and the APU power output follows the energy demand of the electric motor thoroughly. In real life, series hybrids operate as a combined load-leveling loadfollowing series HEV. Their operation is optimized for the nature and length of their duty cycle based on engine and battery efficiency.

Although certain series configurations can eliminate the need of a transmission and/or require smaller battery capacity than EVs, the hardware of the series HEV, in general, is more expensive than that of EVs or conventional vehicles because it requires two electric machines and an ICE. In addition to that the control of it is more complicated than the control of electric or conventional vehicles [17]. 


\subsubsection{Advantages and disadvantages of parallel HEVs}

Parallel HEVs have dual energy stored on board: fuel and electricity, just like series HEVs. However, in a parallel hybrid electric vehicle the engine and the electric motor are coupled in parallel and linked mechanically to the wheels through some type of transmission and a differential. This direct coupling infers that the ICE does undergo significant transients in speed but not in torque as it can be assisted by the electric motor. The speed transients are a drawback from the vehicle's emissions standpoint compared to the series setup. On the other hand the motor can be used to level the torque load making the ICE operate in a more efficient range. Typically ICEs operate more efficiently at higher loads (at moderate speeds). When a low load is required by the vehicle the engine can either be shut off while the motor alone drives the vehicle or the motor can increase the engine load. In turn it supplies extra energy to the batteries to be stored for later use. The greatest advantage of a parallel HEV lies in its performance. Parallel HEVs have the potential to use both their electric motor and ICE as power sources simultaneously for propelling the vehicle resulting in superb performance.

There are two basic types of parallel HEV schemes. In one of them the main power source is the engine and the electric motor assists. In the other one the electric motor is the main power source and the ICE assists. Figure 15 shows the power flow diagram for parallel HEV when the electric motor assists the internal combustion engine. Figure 16 shows the power flow diagram for parallel HEV when the ICE assists the electric motor. 


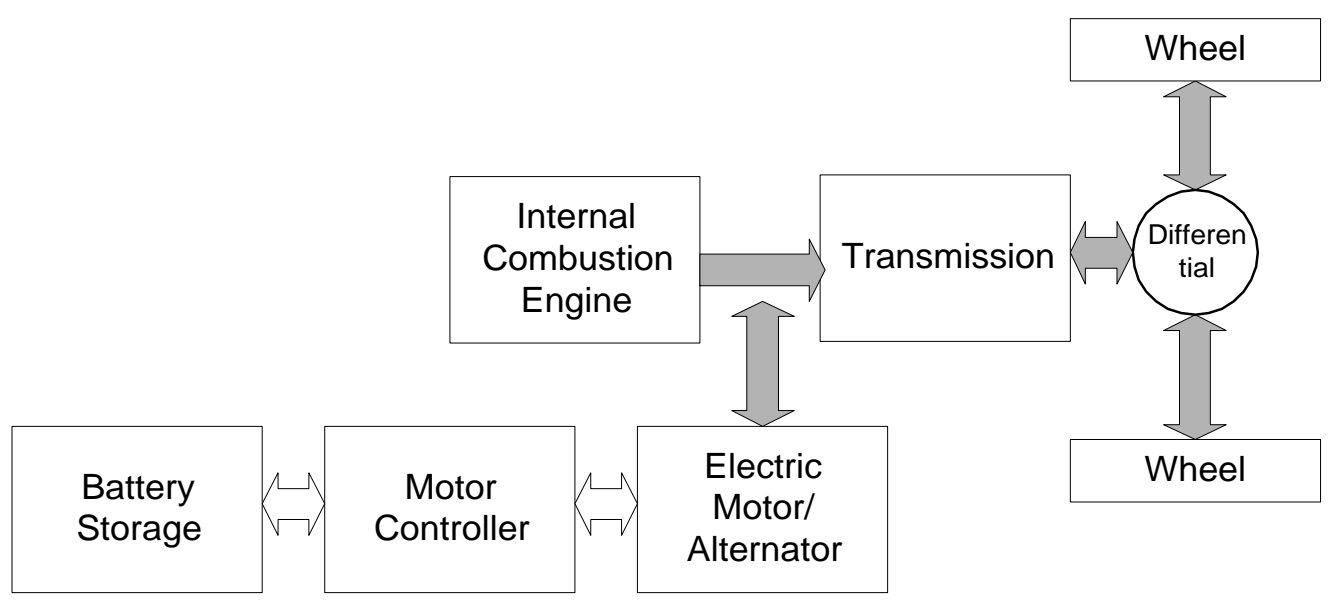

$\square$ Mechanical Energy Flow

Electrical Energy Flow

Figure 15. Power flow diagram for parallel HEV when the electric motor is before the transmission and it assists the internal combustion engine. The electric motor can also be positioned pre- or post-transmission or on a different axle or wheel [17].

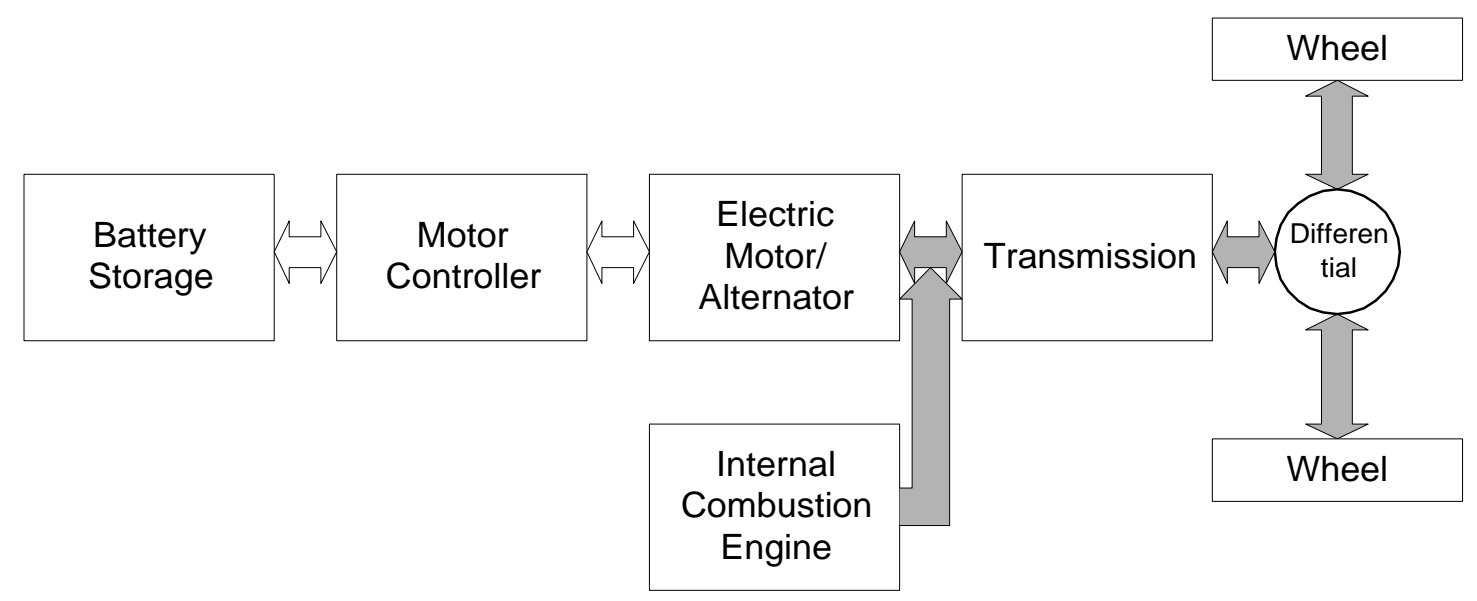

$\square$ Mechanical Energy Flow

$\square$ Electrical Energy Flow

Figure 16. Power flow diagram for parallel HEV when the ICE assists the electric motor. Another version of parallel HEVs is when the electric motor is after the transmission. In that case the inefficiency of the transmission does not affect the power of the motor [17].

The hardware of a parallel HEV is less expensive than that of a series HEV because one electric motor, smaller than that of a series HEV, is sufficient. The control, 
on the other hand, is much more involved since there is physical coupling between the engine and the motor.

\subsubsection{Advantages and disadvantages of combination HEVs}

It is possible to build a vehicle that can be operated either as a series or as a parallel or even some combination of both for different driving conditions. This would utilize the advantages of both the series and the parallel drivetrain types. For example in stop and go traffic the vehicle can operate as an EV or a series $\mathrm{HEV}$, where series vehicles are the most efficient, or it can operate as a parallel HEV when full power is required. There are several ways to create a drive train that has the characteristics of both a series HEV and a parallel HEV but the two main layouts are the series-parallel combination and the planetary combination HEVs.

The series-parallel combination has two electric machines and an ICE coupled through shafts, different drives and clutches. These coupling devices can switch the power sources in such a way that in one instance the powertrain operates as a series HEV and at another instance it operates as a parallel HEV. Depending on driving conditions, the various modes would be selected to utilize the most advantageous individual mode. This however would involve even more components than either a series or a parallel further increasing the size, weight, and complexity of the powertrain and the complexity of the control. Figure 17 shows the series-parallel combination HEV. 


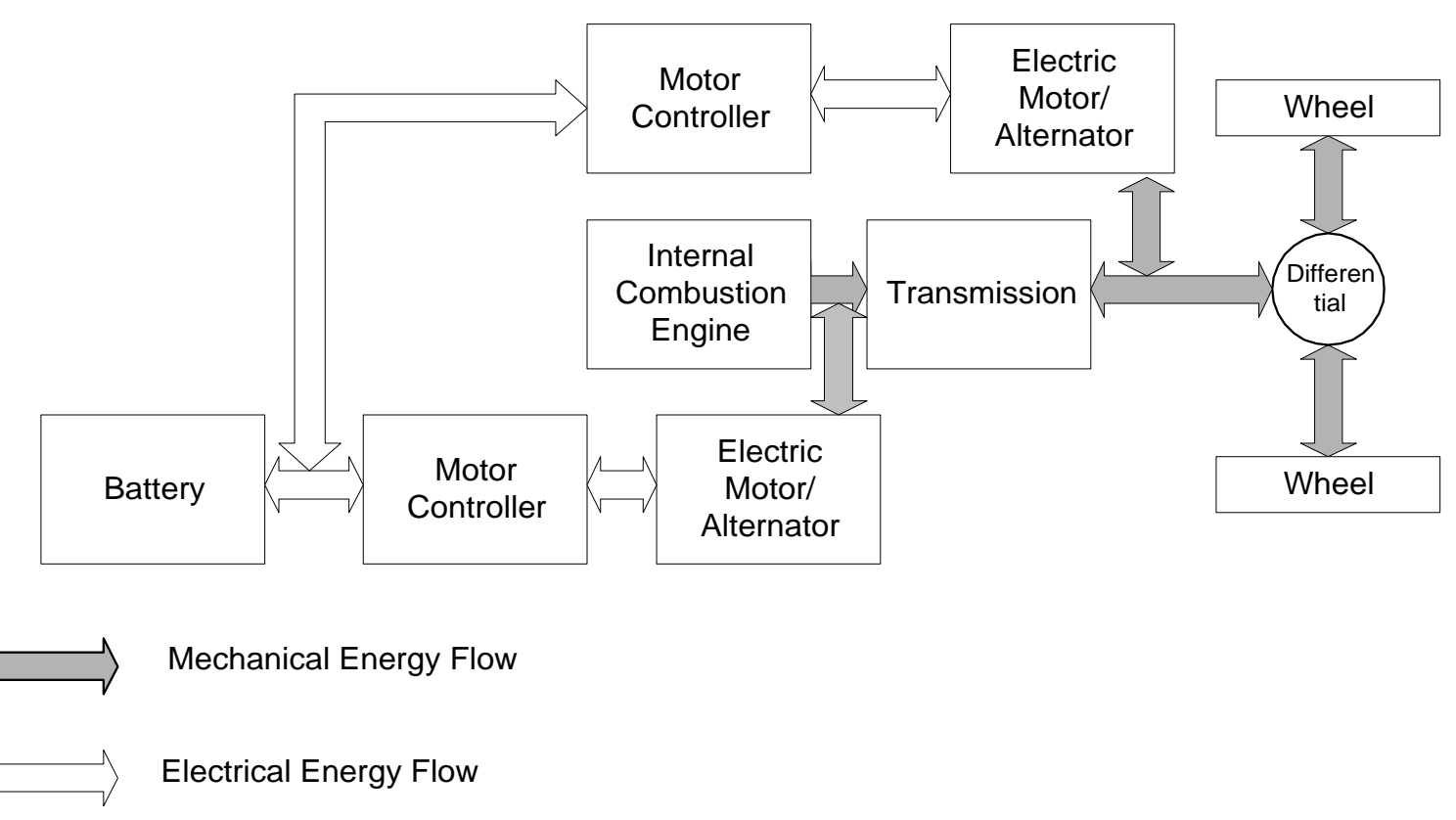

Figure 17. Power flow diagram for the Series-Parallel HEV [17].

The other main design of combination hybrids is the planetary combination hybrid electric vehicle (PC-Hybrid). The PC-Hybrid couples an ICE, an alternator, and a motor via a planetary gear set. Several possible setups of the power source components can be arranged around a planetary gear set [20-23]. One of those versions is employed by the Toyota Prius. The engine is linked to the planet carrier; the alternator to the sun gear, and the output is the ring that transmits the torque to the differential. The motor is also linked to the ring gear so that it is able to add torque through the output shaft to the differential. With this setup there are three degrees of freedom, with the alternator being used to control the extra degree of freedom on the sun. Changes in the alternator operation affect the engine operation yielding total control over the engine at all driving conditions (within reason). Because the alternator controls the torque on the engine, the engine can operate at the most efficient point at each speed of the vehicle. This is not a real advantage in diesel engine applications because diesels' efficiency varies little with partial load operation. However, the less transient operation results in lower exhaust gas 
emissions. Because there is no gear changing involved with the PC Hybrid the engine operation is less transient than that of the parallel configuration resulting in less emissions and higher powertrain efficiency than that of parallel vehicles giving similar performance.

PC-Hybrids are also called power split HEVs because the planetary gear set splits the power of the engine. It basically splits the torque constantly and a smaller portion (approximately $1 / 3$ in the Prius) is transmitted electronically through the alternator, the electric motor, and the differential and a larger portion (2/3) of the torque is transmitted mechanically through the planetary gear set and the differential. This mechanical link results in higher efficiency than that of a series hybrid. In this setup the vehicle acts as a series $\mathrm{HEV}$, only when the vehicle is stationary and just starts moving. All the power from the engine is transmitted through the alternator and the electric motor. As soon as the vehicle starts moving, besides the electrical path, power is transmitted mechanically through the planetary gear set. For the rest of its operation the vehicle works as a combination of a series and a parallel HEV, once again taking advantage of both configurations. If the alternator could be held steady the vehicle would operate as a parallel vehicle. Of course the alternator cannot operate at less than its lowest operating speed because as it loses motion it loses the ability to generate electricity and form torque and control on the sun gear. Thus the vehicle will never operate as a pure parallel HEV unless a mechanical brake is applied on the sun gear.

The hardware cost of the PC-Hybrid is of course more than that of an electric or a conventional vehicle. It needs two electric motors and a smaller engine, compared to a similarly sized conventional vehicle engine, and it eliminates the need for the 
transmission that makes the PC-Hybrid one of the cheapest and most integrated designs amongst combination hybrids and a competitive configuration amongst hybrids generally. The control of the PC-Hybrid is more complicated than that of the series HEV. Figure 18 shows the basic layout of the PC-Hybrid.

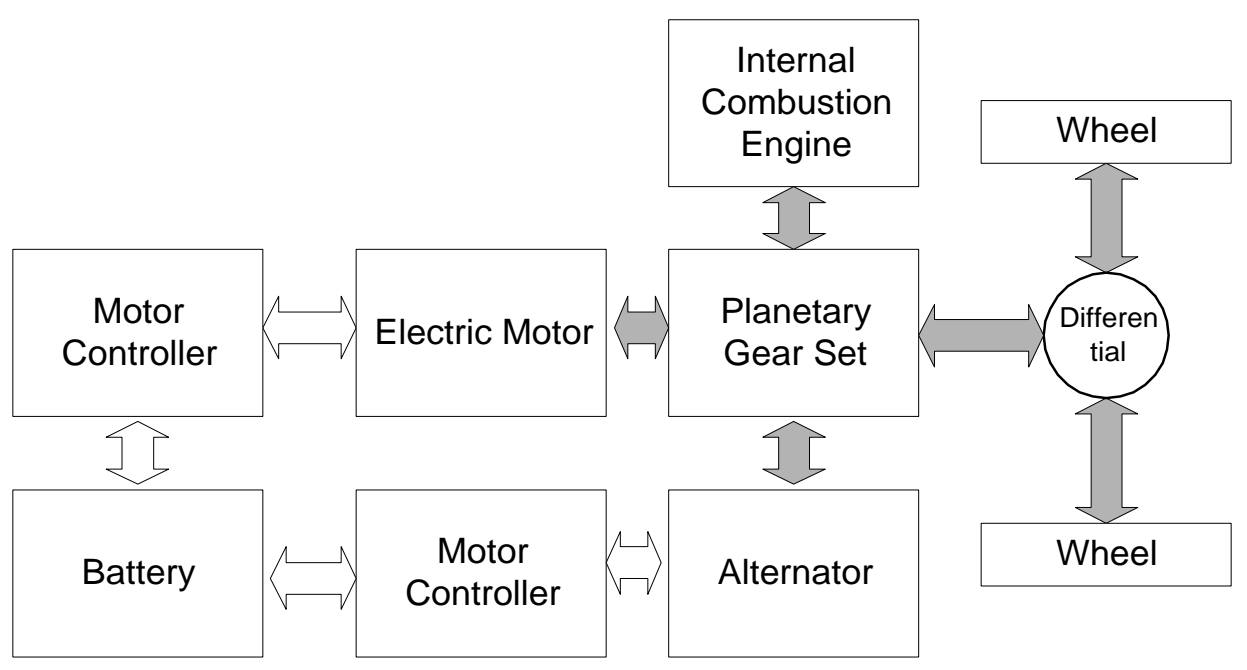

$\square$ Mechanical Energy Flow

Electrical Energy Flow

Figure 18. Power flow diagram for the planetary combination hybrid [17]. 


\subsubsection{Advantages and disadvantages of fuel cell hybrids}

Fuel cell hybrids are similar to series hybrids in configuration. The vehicle itself is propelled by an electric motor, or motors but the APU is a fuel cell instead of an ICE/generator set. There are two kinds of fuel cells under development: the proton exchange membrane (PEM) type and the solid oxide fuel cell (SOFC). The key component of the PEM fuel cell is a catalyst-coated (platinum) membrane that conducts positively charged hydrogen ions only. When oxygen and hydrogen are introduced in the fuel cell on the opposite sides of the membrane the catalyst coating separates the valency electrons from the hydrogen molecule resulting in a positively charged hydrogen ion that can penetrate through the membrane to join the oxygen on the other side and form water. The electrons are forced to go around the membrane to join the oxygen and complete the bond of the water molecule [24]. The current resulted by the electron flow can be used to drive the electric motor or it can be stored in the battery.

The solid oxide fuel cell operates on the same principle. The difference lies in the materials participating in the reaction. The membrane conducts positively charged oxygen ions only therefore it is the oxygen ion that penetrates through the membrane. On the other side of the membrane any kind of carbon-based fuel can be used to attract the oxygen. The fuel gets oxidized by the oxygen and forms $\mathrm{CO}_{2}$ and $\mathrm{H}_{2} \mathrm{O}$. Electrons are again forced to flow around the membrane resulting in a current flow that can be used to drive the vehicle [103, 104]. SOFC basically uses carbon-based fuel just like an IC engine. It can use a wide range of fuels and because it operates at moderate temperatures it does not produce NOx and because it eliminates conventional burning it does not produce PM. It is also quieter and less vibrating than an IC engine. 
PEM fuel cells do not use carbon-based fuel. They use hydrogen that is present on Earth, as water, in large quantities therefore they might be the long-term solution for the projected oil crisis. The only tailpipe emission they produce is water. However, hydrogen is difficult to store on board and that seems to be the major showstopper for PEM fuel cells these days. Hydrogen cannot be stored in large enough quantities in gaseous form to provide the required range for the vehicle. To store it in liquid form requires high-pressure low temperature cryogenic tanks that are a very expensive form of storage. Until the storage problem is solved parallel research was done in the direction of gaining hydrogen from so-called hydrogen carriers, which are usually hydrocarbons, from oil products [105]. Applying hydrocarbon-based fuel would make the PEM no more beneficial than the SOFC for using oil based product and emitting $\mathrm{CO}_{2}$.

\subsection{Benefits and drawbacks of hybridization}

Hybridization provides two major benefits: decreased exhaust emissions and increased fuel economy [17]. These benefits are the results of downsizing the engine, less transient operation of the engine, and the application of regenerative braking that reduces average power demand. While in conventional vehicles the engine alone has to meet the instantaneous power requirement to propel the vehicle, in HEVs a smaller engine is sufficient with added power from the electric motor to provide the same performance. The electric motor adds the extra power using the electrical energy stored in the batteries. At the same time, the added power from the electric motor makes it possible for the engine to operate in a less transient manner. This results in fuel economy improvement as well as lower exhaust emissions. Probably the most beneficial feature of hybrid and electric vehicles in city driving is regenerative braking. During regenerative 
braking the controller switches the electric motor to operate in generator mode providing the braking torque on the wheels and generating electricity at the same time. The generated electricity is stored in the batteries and can be used for the next acceleration.

These benefits do not come for free in hybrids, though. The integrated ICE and electric motor drive system makes a heavier and more complicated powertrain than that of a conventional vehicle. It costs more money, involves more components with possible failure and increases control complexity resulting in increased price. At the present oil prices the savings on fuel do not compensate for the additional purchasing and maintenance cost involved with hybrid vehicles. This means that fuel prices will have to go much higher before consumers will decide to pay the higher price for hybrids.

\subsection{Hybrid development}

Although buying and maintenance still cost more for a hybrid than for a conventional vehicle, the fact that every major car manufacturer works on hybrid designs suggests that there is going to be interest on the market for these vehicles in the close future. R \& D departments of different auto manufacturers have different goals, market targets, and different HEV configuration designs. In 1998, Honda and Toyota started the mass production of the Insight and Prius, respectively. Today, September 2004, they are still the only two manufacturers that produce hybrid vehicles commercially available on the market. This part of the chapter attempts to show some of the major manufacturers' design efforts and to show the trends in hybrid vehicle development. 


\subsubsection{Honda}

In 1998, Honda was the one of the first two companies on the hybrid market. Their vehicle was the Insight, which is a so-called mild hybrid parallel HEV. As a mild hybrid the Honda Insight has a low degree of hybridization meaning its electric motor is quite small (10 kW, permanent magnet) compared to its fuel converter (49 kW, $995 \mathrm{cc}$, gasoline). This configuration gives an increased fuel economy, compared to the conventional version, for utilizing regenerative braking and smoothening engine operation. However, the vehicle will still operate as a conventional vehicle if something goes wrong with the electrical system. This trend of "milbridization", meaning producing milbrid (mild hybrid) vehicles, can be seen in the case of the future design of several manufacturers. The Insight has Ni-MH (nickel-metal-hydride) batteries as an energy storage device at a nominal voltage of $144 \mathrm{~V}$ and capacity of 6.5 Ah. The electric motor occupies the space of the flywheel fulfilling the role of the flywheel besides motoring and regenerating when needed. The EPA fuel economy of the vehicle is 61/70mpg city/highway. The car weighs $1856 \mathrm{lb}$ [45]. Honda also made a hybrid version of the Civic based on an upscaled version of the Insight hybrid system.

\subsubsection{Toyota}

Toyota, also in 1998, entered the market with a hybrid vehicle: the Prius. The Toyota Hybrid System (THS) in the Prius incorporates a high expansion-ratio gasoline engine (51 kW, $1497 \mathrm{cc}$ ), a permanent magnet electric motor (30 kW), and a permanent magnet alternator $(14 \mathrm{~kW})$ via a planetary gear set to form a planetary combination hybrid. The energy storage is a Ni-MH battery pack with $273.6 \mathrm{~V}$ nominal voltage and 6.5 Ah capacity. The EPA fuel economy of the Prius is 52/45-mpg city/highway. The 
Prius weighs $2765 \mathrm{lb}$. [46] Toyota also developed a four-wheel drive minivan based on an upscaled version the THS involving a CVT for high torque demand situations [46], and Lexus is coming out with a luxury SUV based on the Toyota Hybrid System. Another development at Toyota is a $32 \mathrm{~V}$ starter-alternator system for an option in Toyota’s other milbrid products [46].

\subsubsection{Ford}

Six years after Toyota and Honda started the mass production of their hybrids, Ford is planning to enter the market with the hybrid Escape in 2004 as the first commercially available hybrid SUV. It is a planetary combination hybrid incorporating a high expansion ratio four-cylinder engine, a $65 \mathrm{~kW}$ permanent magnet electric motor, and a $28 \mathrm{~kW}$ alternator. The Ni-MH battery pack operates at $300 \mathrm{~V}$. Ford claims that the hybrid Escape will run 40 miles on a gallon of gas in city traffic [47].

\subsubsection{General Motors (GM )}

General Motors has developed the ParadiGM system for the SUV market. The ParadiGM system is a mild parallel hybrid incorporating two electric motors. One is pre and the other is post transmission with a manual gearbox in between. GM will offer inline four and V-6 engines with the ParadiGM drivetrain. The electric motors operate at $42 \mathrm{~V}[48]$.

\subsubsection{Hybrid electric high mobility multipurpose wheeled vehicle (HHMMWV)}

The hybrid electric high mobility multipurpose wheeled vehicle, known as the hybrid Humvee, is an application of the series configuration using a 1.9-liter Volkswagen 
TDI engine and a $55 \mathrm{~kW}$ UQM permanent magnet generator as its APU and four $75 \mathrm{~kW}$ electric motors, one for each wheel, to propel the vehicle. The battery pack is built of advanced lead-acid batteries with 85 Ah capacity and $288 \mathrm{~V}$ nominal voltage [43]. The Humvee takes advantage of the low noise, the low heat profile, and the lack of visible exhaust gases that the EV mode provides in stealth situations. The vehicle accelerates 0$50 \mathrm{mph}$ in 7 seconds and climbs a 60\% grade at $17 \mathrm{mph}$ [43]. The conventional Humvee takes 14 seconds to accelerate $0-50$ and climbs the $60 \%$ grade at $7 \mathrm{mph}$. It seems to be just a bonus that the hybrid Humvee doubled the fuel economy of that of its conventional version to $16 \mathrm{mpg}$ [42].

\subsubsection{Heavy-duty sector}

Several hybrid electric drivetrains were developed in the heavy-duty sector [100 102]. Nova and Orion buses are already in service in urban areas with Allison and Lockheed-Martin hybrid drivetrains respectively. Both buses are series hybrids. Allison offers the same drivetrain for heavy-duty trucks and Lockheed offers its drivetrain for military vehicles. Allison is also working on a second-generation hybrid powertrain, which is a planetary combination hybrid for buses.

\subsection{Conclusions}

After seeing the basic hybrid vehicle layouts and their application in vehicles, it can be concluded that there are only three configurations that are favored by manufacturers and are manufactured or being proposed for manufacturing in the close future. These three configurations are the mild hybrid parallel, planetary combination hybrid, and series hybrid configurations. The series layout was only used for heavy-duty 
application. The mild hybrid concept is cost effective and relies on the engine most of the time. It is market-safe for experimenting, taking moderate advantage and risk of the hybrid concept. The planetary combination hybrid offers a versatile use of the vehicle and performs well in city traffic as well as on the highway. It has better efficiency than a series hybrid even in stop and go city traffic. It clearly is the most efficient configuration. Although the series hybrid is not as efficient as a planetary combination hybrid, it has its merits, which are simplicity and relative ease of control. Furthermore, a series hybrid can easily be converted to a fuel cell hybrid when fuel cells and hydrogen tanks will be market ready. 


\section{Linear engine}

This chapter introduces the linear engine and presents the literature that discusses the topic. It groups the linear engines according to the location where the combustion happens, ignition type, principle of operating cycle, number of strokes per cycle, number and configuration of cylinders, and the application of the linear engine. The name, linear engine, speaks for itself. It reveals an engine with linearly moving components only. The linear engine does not have a crankshaft. This eliminates the rotating components of a slider-crank mechanism. In a conventional single cylinder slider-crank engine there are about 50 components, of which 28 undergo linear motion and 22 undergo rotating motion [39]. The connecting rod does not belong to either group for having one end reciprocating linearly and the other doing circular motion. The rotating components are eliminated in the linear engine resulting in decreased complexity, lower manufacturing cost, and almost maintenance-free operation. Since there is no side thrust on the piston it has decreased wearing effect on the cylinder compared to a slider-crank engine. Furthermore, with the proper selection of materials the linear engine does not need lubrication. This will eliminate the need of oil changes and decrease maintenance expenses. The linear engine is also called a free-piston engine in some literature because there is no physical linkage that would constrain the piston's motion. The piston's or the translator's motion is determined by the equilibrium of forces acting on the translator. The only mechanical connection that is present is the contact between the pistons and the cylinders. 


\subsection{The first attempts}

The first steam-powered equipment, of which a written description exists, was a temple door actuator in the ancient Alexandria in the $1^{\text {st }}$ century AD. Before the ceremony started, the priest made fire on the altar that heated a closed container underneath the fireplace. The steam developed drove the water out of the container to a bucket and the weight of the bucket opened the door signaling the beginning of the ceremony [25]. Figure 19 shows the schematics of the steam operated temple dooropening device.

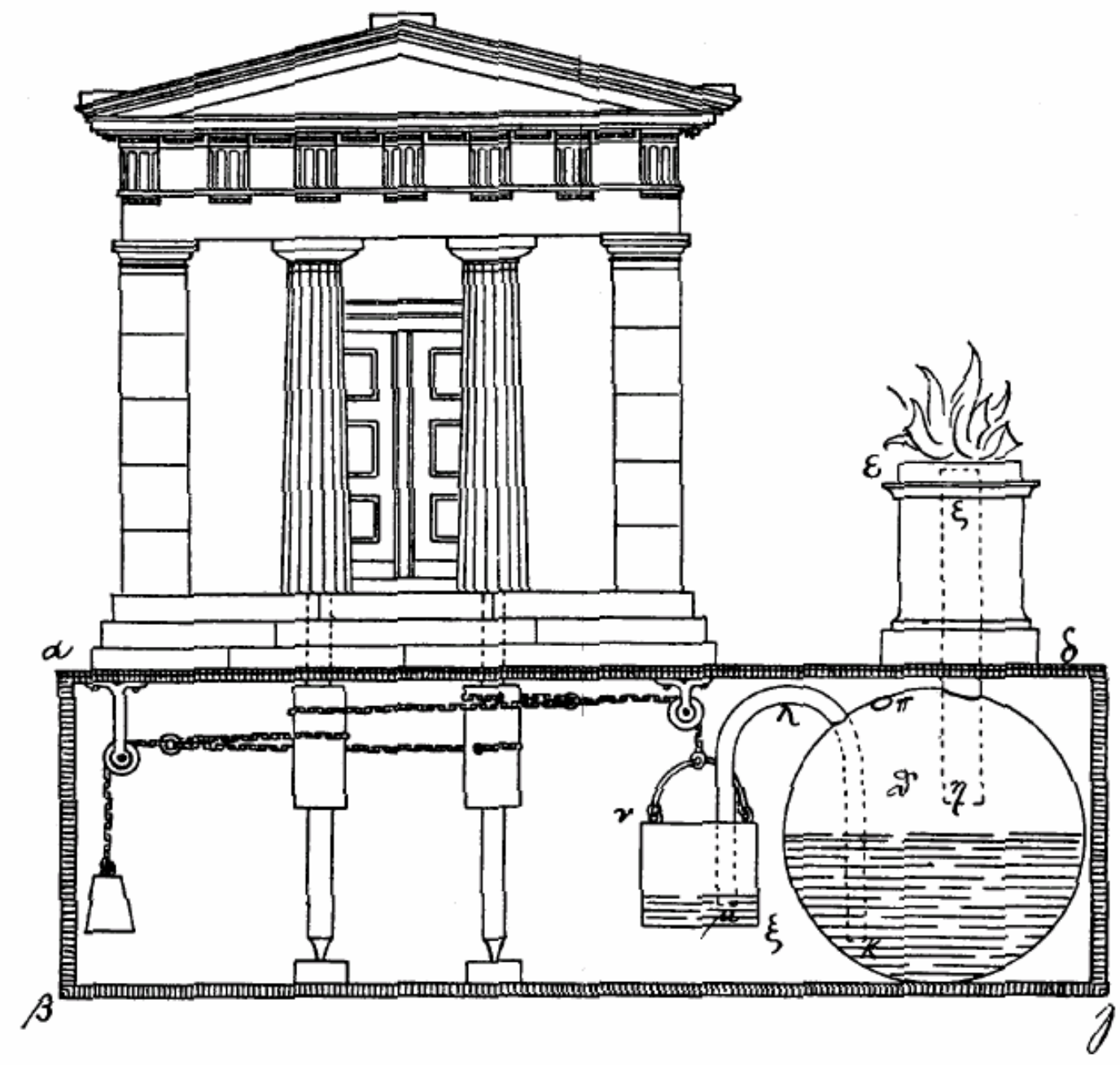

Figure 19. Steam operated temple door from the $1^{\text {st }}$ century [25]. 


\subsection{External combustion versus internal combustion}

Since the time of the steam powered temple door, numerous engines and engine variations were invented. Engines can be grouped based on where the combustion happens, namely external and internal combustion engines. In external combustion engines the combustion takes place outside of the cylinder and some medium transfers the energy to the working cylinders. In internal combustion engines the combustion takes place in the cylinder and the expanding gas itself is the working fluid.

\subsubsection{External combustion engines}

One of the most important external combustion engines was James Watt's steam engine that revolutionized the industry in England at the end of the $18^{\text {th }}$ century. James Watt's steam engine, patented in 1769, used a boiler to transfer water to steam and a valve control system to direct the steam into and out of the cylinder over or under the piston alternately that resulted in the piston's reciprocating motion. Watt's first steam engine was a linear machine that used gravity as well as the power of steam to achieve motion. Figure 20 shows Watt's steam engine [25]. 


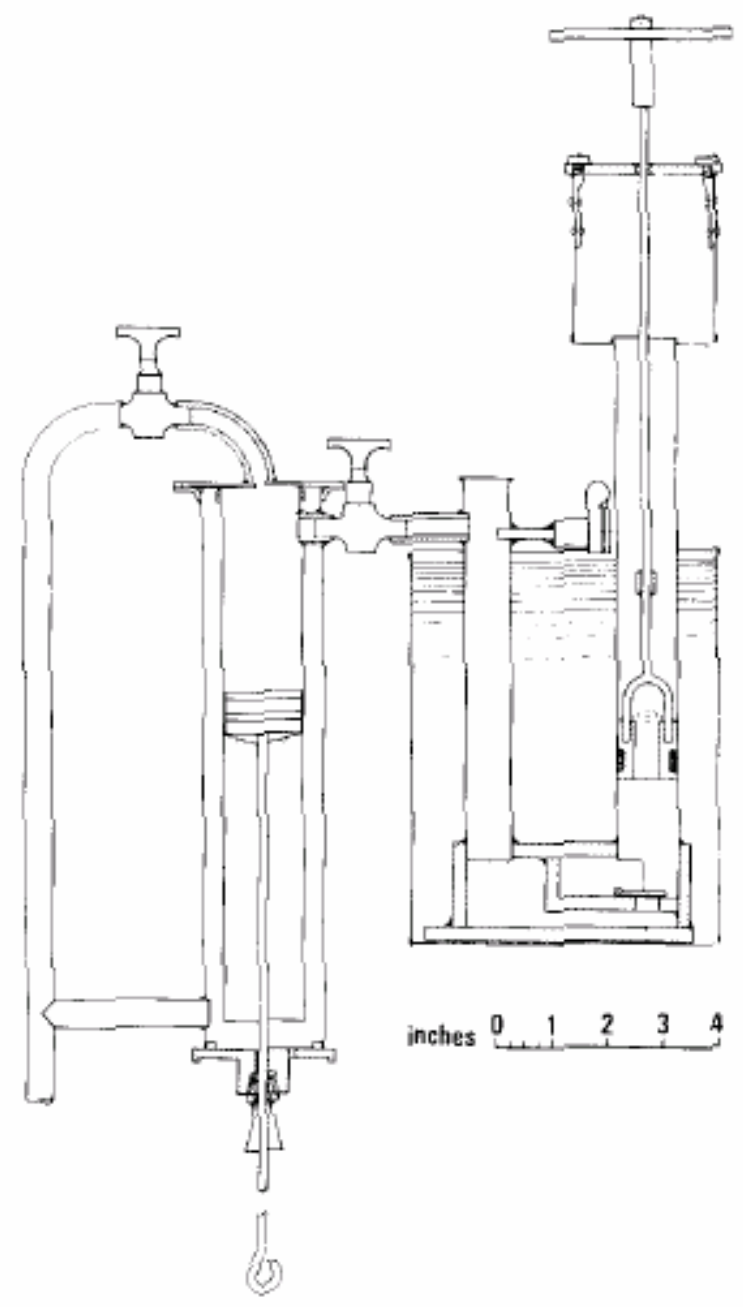

Figure 20. James Watt's steam engine [25].

It is inevitable that the particular design was a linear engine. In later steam engines the linear alternating motion of the piston was converted to rotary motion using a slider crank mechanism. Although the best-known and most recent application of the steam engine, the steam driven locomotive, was used as the main transportation for over a century until about the 1970's, steam engines are historical subjects today.

Another example for external combustion is the "economizer" as Stirling called the engine he patented in 1816. Figure 21 shows Stirling's engine. 


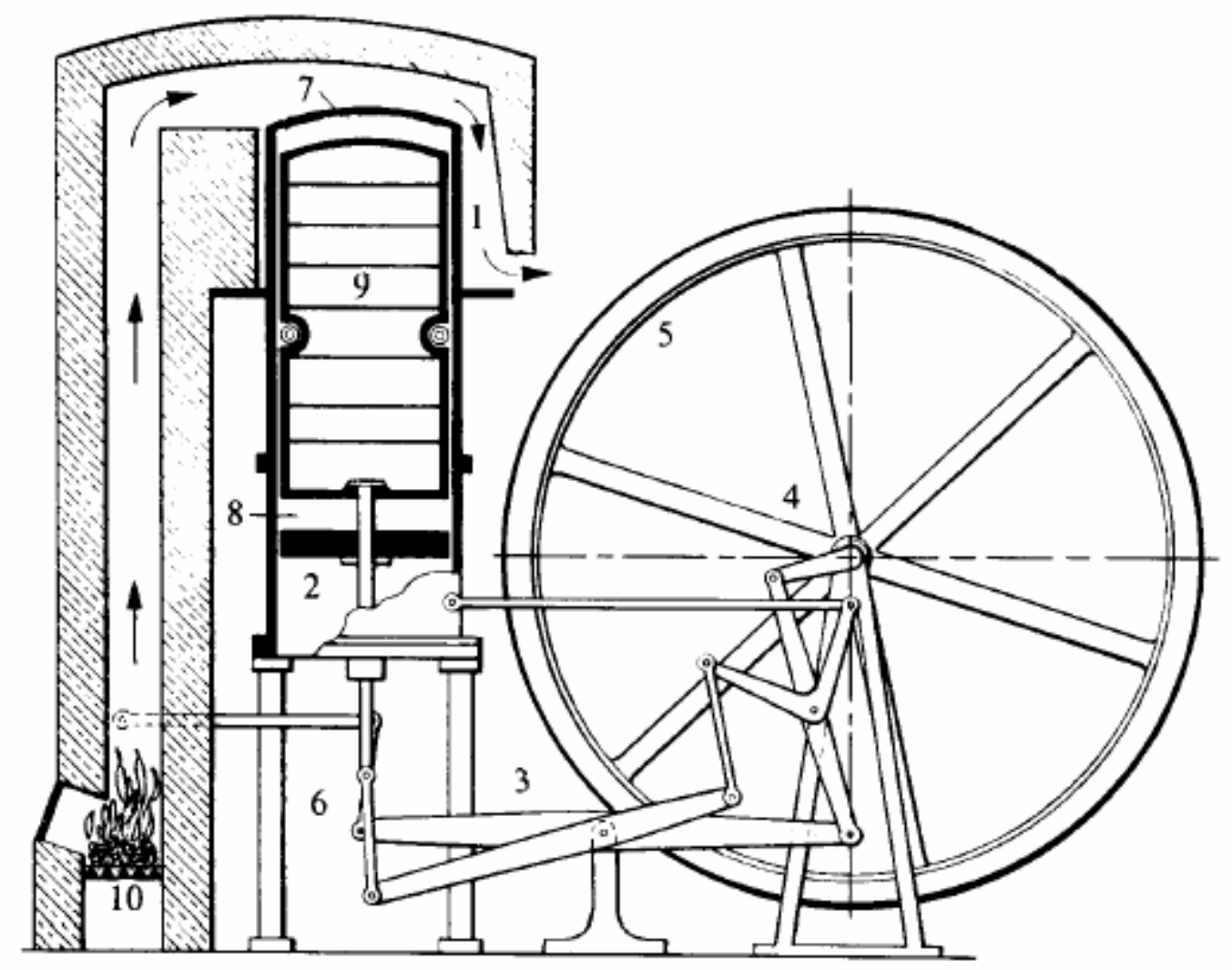

Figure 21. Stirling's engine [26].

The engine has two moving components in the cylinder: the plunger, or displacer (top), and the power piston (bottom). A linkage-flywheel mechanism connects the two restricting their motion relative to each other. The design consists of a hot and a cold chamber above and below the plunger respectively. Combustion gases heat the hot chamber (7) and air cools the cold one (8). In later designs, after 1828, Stirling applied water-cooling around the cold chamber. There is a gap between the plunger and the cylinder wall that lets the air flow between the two chambers. As a result, the middle section of the cylinder and the piston itself acts as a regenerator pre-heating or precooling the flowing air. The plunger is centered in the cylinder by wheels (a). For easier understanding Hargreaves [27] presents the Stirling engine’s operation in a discontinuous manner broken down to 4 stages. Figure 22 shows the model at the 4 stages of operation. 


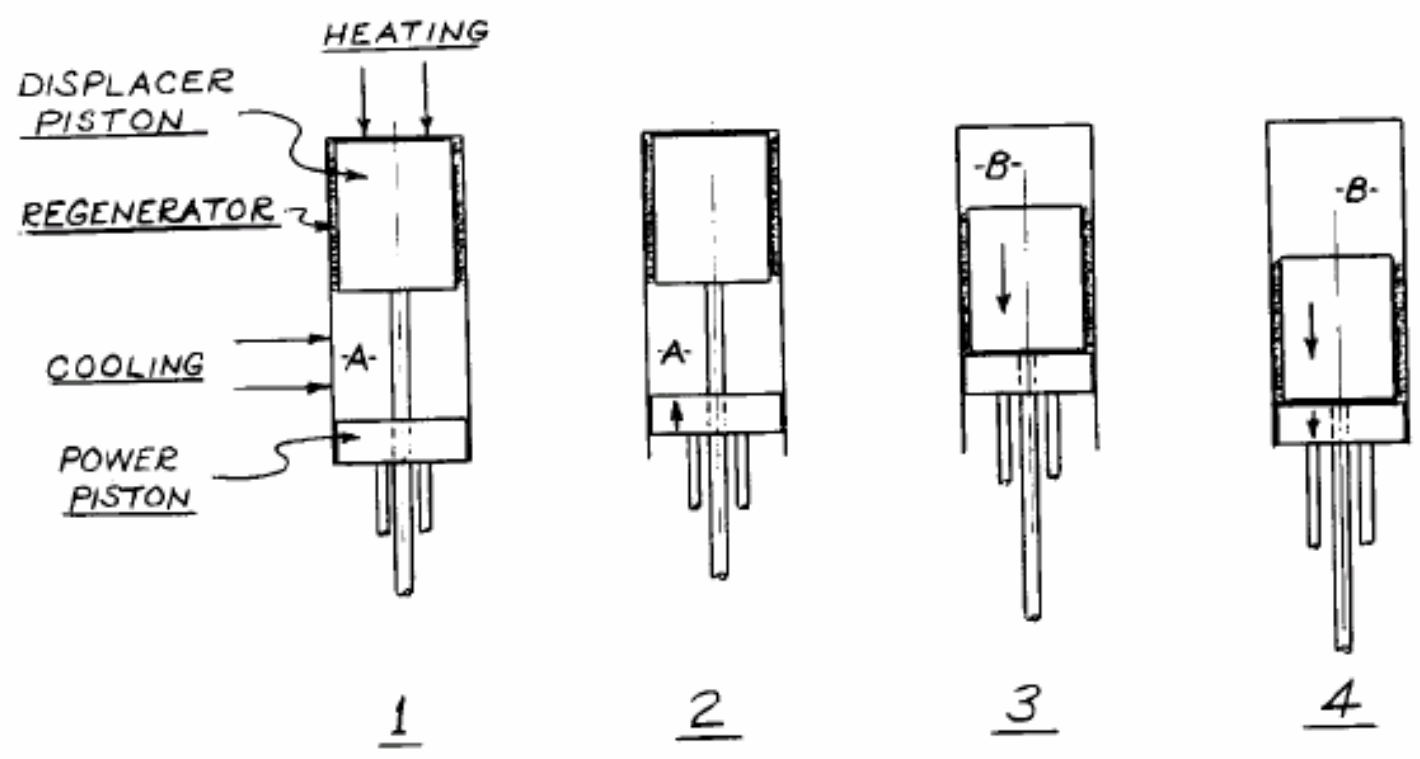

Figure 22. The four stages of operations of the Stirling engine [28].

The four stages of operation are:

I, Power piston is in lowest position; all the air is in the cold space.

II, Displacer is still in highest position but power piston has moved upwards, compressing the air while it is at low temperature.

III, Power piston has remained in its highest position but displacer has moved about halfway downwards, transferring the air from the cold space via the regenerator into the hot space.

IV, The hot air has expanded, pushing the power piston downwards, the displacer moving with it to the lowest position. While the power piston remains there, the displacer will then move upwards, transferring the air from the hot space, via the regenerator, to the cold space as in stage-I, completing the cycle.

Countless Stirling engines were designed and built in the last 185 years.

References [26-31] list several of them for various applications including automotive power sources. Stirling engines are appealing for automotive use for their low noise level, versatility of fuel use, and smooth operation. However, they are not quick to respond to control and the heat exchangers of the engine are quite bulky to ensure high 
efficiency, and that makes them undesirable for automotive use but excellent candidates for stationary power generation systems.

The principles of the Stirling engine were applied to the new concept of linear or free piston engines as well. References [31-38] show mathematical models, simulations, and prototype designs of free piston Stirling engines. The linkage-flywheel mechanism is eliminated in free piston Stirling engines and an air spring is placed between the plunger and the piston. The result is a "machine in which the motion of the reciprocating elements to accomplish the thermodynamic cycle are effected by fluid forces and by the dynamical, fluidic interaction of the components” [26]. Beale et al., in their paper [32], explain the operation of the free piston Stirling engine in four stages similarly to that of Hargreaves [27]. Figure 23 shows the schematics of the engine at four stages of operation. 

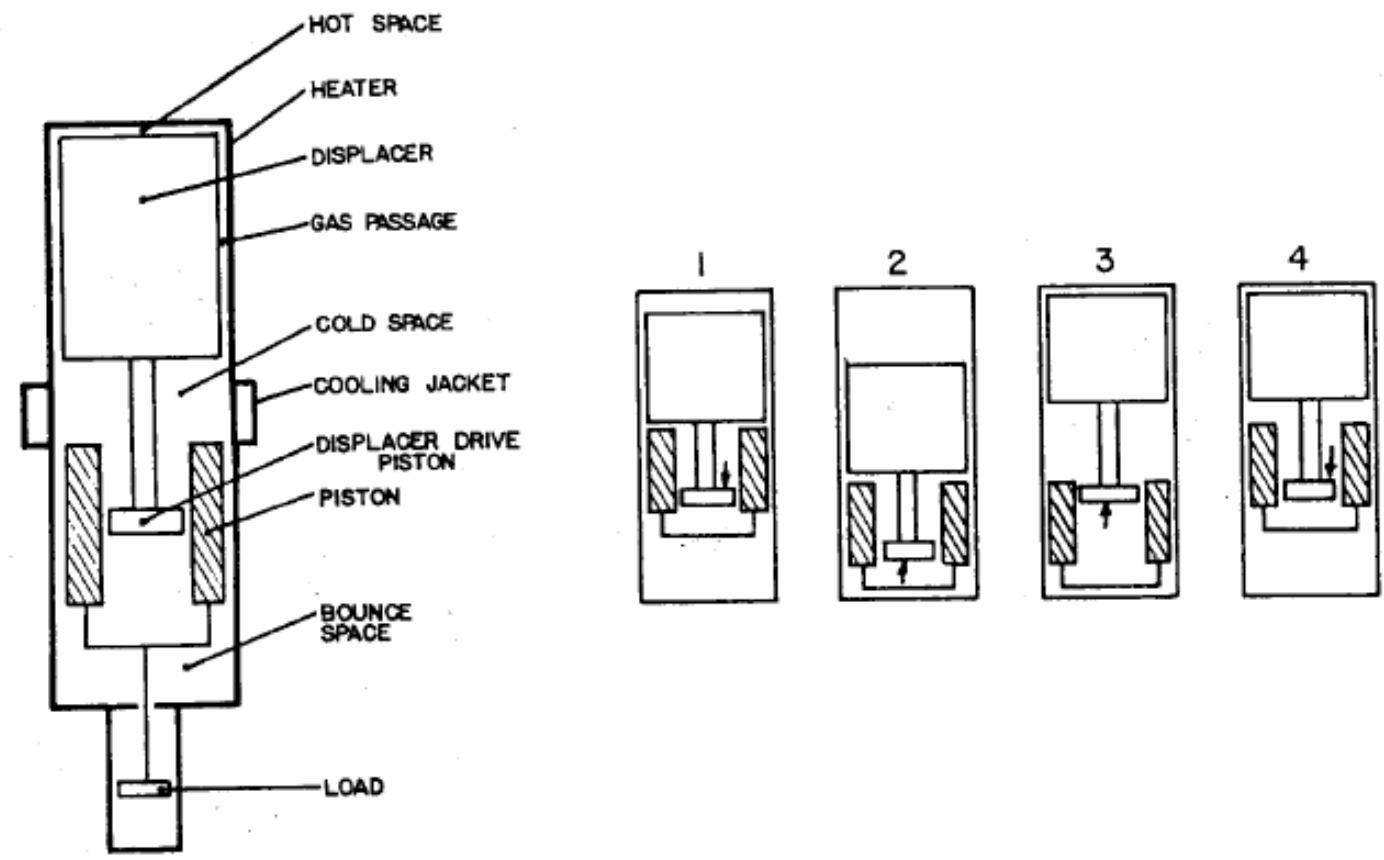

Figure 23. The four stages of operation of the free-piston Stirling engine [32].

I-II Piston expands working gas. Displacer is on piston.

II-III Pressure in bounce space is greater than pressure in working space. This forces displacer toward hot space, and working gas to move into cooled space. Pressure dropped.

III-IV Piston driven into working space by higher bounce space pressure.

IV-I Displacer is driven toward cold space by working space pressure higher than bounce space pressure.

Free piston Stirling engines project a very promising power source because of their versatility in fuel use. Any fuel can be used in the burner. Dochat [36] introduced a solar thermal powered Stirling engine prototype for aerospace application. The hot chamber of the engine was positioned in the focal point of a parabolic mirror and worked between 300 and $500{ }^{\circ} \mathrm{C}$ using solar energy only. The unit produced 500-1100 watts. This engine could be regarded as a zero emission power source with low maintenance as it has just a few moving components subjected to friction. 


\subsubsection{Internal combustion}

While in external combustion engines the combustion heats the working fluid that converts thermal energy to kinetic energy, in internal combustion engines the burning and expanding gas itself is the working fluid. This results in higher pressures and temperatures in the cylinder and a more compact design than in external combustion engines. ICEs have higher power-to-weight and power-to-volume ratios compared to external combustion engines. Internal combustion engines became the primary power source for vehicles because their compact design is very suitable for automotive application.

The first successful internal combustion engine was a gas engine considered to be made by Étienne Lenoir in Paris 1859. Although the engine worked, it was only in 1878 when it became commercially available due to the work of the German inventor Nikolaus Otto. In 1892 Rudolf Diesel, also of Germany, invented the diesel engine. At first, it was fine coal powder that Diesel injected in the cylinder but later he used oil products.

Liquid fuel was used exclusively in diesel engines until 1893, when two Hungarian scientists Bánki \& Csonka invented the carburetor. This made the liquid fuel available for the Otto engine as well [16]. Since that time the two widely used internal combustion engine types have been the spark ignition (gasoline) and the compression ignition (diesel) engines. 


\subsection{Spark ignition versus compression ignition}

The two major fuel types for engines today are gasoline and diesel fuel. Although in some areas compressed natural gas (CNG) and liquefied petroleum gas (LPG) are also used, a worldwide refueling infrastructure exists only for gasoline and diesel fuel. The two fuel types have spread around the world driven by two different principles of operation for engines: spark ignition (SI) and compression ignition (CI) engines.

The spark ignition engine takes in fuel and air mixture and following compression a spark ignites the charge. The intake charge is usually stochiometric in conventional engines. However, lean burn engines are under development with the goal of increased efficiency but results vary [94 - 96]. The engine is controlled through a throttle valve that limits the mass of air taken into the cylinder by limiting the pressure in the intake manifold. Fuel to air ratio does not vary much by design.

Diesel engines take in air, and fuel is injected in the cylinder around TDC where it autoignites under high pressure and temperature caused by the compression. Diesel fuel is required to have a high cetane number, which represents a high affinity to autoignite. The engine power is controlled by the amount of fuel injected into the cylinder. Diesel engines operate at low equivalence ratios most of the time. They are lean burn engines.

Besides the fact that diesel fuel has a slightly higher density and higher heating value, than gasoline [92], three things cause the major efficiency difference between engines: the presence of throttle in an engine that results in pumping losses, the equivalence ratio at which the engine is operated, and the compression ratio of the engine. In spark-ignited engines the throttle is part of the engine control system under 
part load conditions causing a major loss of efficiency. In compression ignition engines the lack of throttle results in higher efficiency than that in spark ignited engines so it can be concluded that a throttle is an undesired component of the engine.

Efficiency is also the function of compression ratio and the equivalence ratio. Heywood [91] shows a proportional relationship between an engine’s efficiency and its compression ratio up to 15:1. In spark-ignited engines the compression ratio is limited by knocking, which is the fuel's uncontrolled auto ignition. Knocking is undesirable for causing uncontrolled burning that results in high stress on the engine components as well as in efficiency loss. In compression ignition engines, on the other hand, the maximum compression ratio is only limited by component strength, heat loss, and blowby. Heywood [91] shows an inversed proportionality between efficiency and equivalence ratio due to the higher heat loss and blow-down at operating regions of increased equivalent ratio. Spark-ignited engines usually operate at stochiometric fuel/air ratio to ensure flame propagation and complete burning in the cylinder. Cylinder averaged equivalence ratio in compression ignition engines, on the other hand, can be decreased until the engine stalls and it would still result in proper burning.

The presence of a throttle, the limited compression ratio, and the higher equivalence ratio lead to an efficiency loss in spark-ignited engines, while the lack of throttle, the higher compression ratio, and the lower equivalence ratio yield relatively higher thermal efficiency in compression ignition engines when compared. Both spark ignition and compression ignition were applied to the linear engine concept in the past. Clark et al. [83] presented a spark-ignition linear engine and compression ignition linear engines were discussed in several references [61, 65, 80]. 


\subsection{Direct injection versus homogeneously charged compression ignition}

Increasing engine efficiency has always been the goal of researchers working on engine development. The maximum theoretical efficiency was defined by the constantvolume burning cycle, which is the ideal Otto cycle. As a result of trying to reach ideal Otto cycle efficiency, homogeneous charge compression ignition (HCCI) engines have been developed [97 - 99]. Besides HCCI, the most advanced compression ignition (CI) engine technology applies common rail direct injection systems [93]. The weighting factors between the two advanced technologies are efficiency, exhaust emissions, and controllability over broad torque and speed ranges. HCCI engines take in a lean, homogeneous air-fuel mixture. At around TDC, at elevated pressure and temperature, the homogeneous charge autoignites. The fuel is burned everywhere in the cylinder at the same time approaching the theoretical Otto cycle and yielding high efficiency. From the emissions stand point, HCCI technology reportedly reduces NOx emissions significantly and slightly increases unburned HC and CO compared to direct injection technology. Gray et al. [98] report 98\% NOx reduction. Common rail direct injection yields lower efficiency than HCCI in engines as the burning is not instant because the charge is heterogeneous. In the case of heterogeneous charge the burning happens on the outer surface of the injection spray because the inside of the fuel cloud is too rich to burn. The burning at the perimeter of the fuel cloud results in swirl in the cylinder that mixes the fuel and air content. This lets the rest of the fuel burn. The burning in DICI engines takes longer time that in HCCI engines. NOx emissions are higher due to the extreme temperatures occurring at the flame fronts and to longer exposure to heat. The disadvantage of the HCCI technology is that the engine performance is limited by 
knocking and misfire and leaves a very narrow range of operation in conventional engines. Common rail direct injection, on the other hand is fully developed and controllable over the entire range of engine operation. Research on HCCI technology shows promising results but the control of HCCI engines is yet to be developed. Van Blarigan et al. [74] showed an HCCI linear engine and Achten [60] showed a common rail diesel linear engine.

Figure 24 shows the pressure-volume diagram for constant-volume, limitedpressure, and constant-pressure ideal gas standard cycles. Efficiency is equal to the area enclosed by the P-V curve. The HCCI engine approaches the constant-volume cycle and the common rail diesel lies somewhere between the limited-pressure and the constantpressure cycles. 


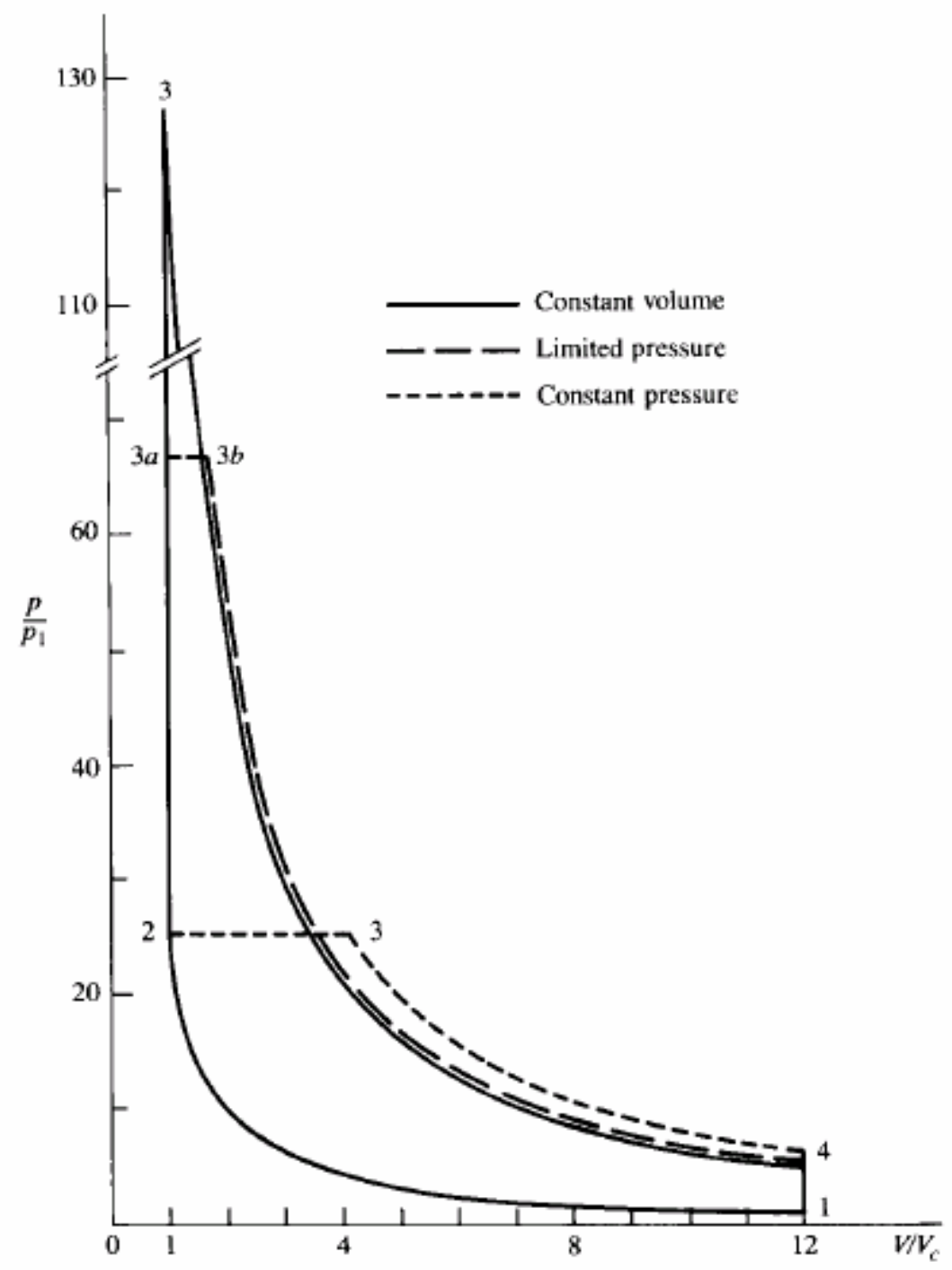

Figure 24. Pressure-volume diagram for constant-volume, limited-pressure, and constantpressure ideal gas standard cycles [91]. 


\subsection{Four stroke versus two stroke}

The two main principles of operation for internal combustion engines are fourstroke and two-stroke operation. The strokes of the four-stroke engine, sequentially, are

- intake

- compression

- combustion

- exhaust

The four strokes happen in two revolutions of the crankshaft, in a conventional slider crank engine, resulting in one power stroke in two revolutions per cycle. Intake and exhaust valves control the scavenging process. The valve operation is synchronized with the piston's motion through the crankshaft, a timing belt or chain and pulleys. The charge enters the cylinder through the intake valve while the piston moves downward. When the piston reaches bottom dead center (BDC) the intake valve closes and the piston starts its way towards top dead center (TDC) compressing the charge above it. The charge is a mixture of air and fuel in a conventional gasoline engine and pure air in a diesel engine. In modern engines exhaust gas is recirculated to decrease NOx emissions so the intake is air-exhaust mixture. Like regular diesel engines, a direct injection gasoline engine takes pure air in and fuel is injected directly to the cylinder at close to TDC. At TDC a spark ignites the charge in a gasoline engine. In a diesel engine fuel is injected into the air that is at high temperature, as a result of the high pressure increase, and autoignites. The fuel burns and the expanding gases push the piston down providing power. At BDC the exhaust valve opens letting the burned gases exit the cylinder as the piston moves upwards. At around TDC the exhaust valve closes the intake valve opens completing the four-stroke cycle. 
The two-stroke operation differs significantly for gasoline and diesel engines.

The strokes of the most typical two-stoke gasoline engines are

- Scavenging and compression in the cylinder. Intake in the crankcase.

- Combustion and exhaust in the cylinder. Crankcase pressurization.

The two-stroke gasoline engine uses intake and exhaust ports and its piston to control scavenging in the cylinder. The charge (air-fuel mix) enters the crankcase below the piston while the piston itself moves upward and compresses the air above it. Both the intake and exhaust ports are closed. At TDC a spark ignites the charge and the expanding gases push the piston towards BDC providing power. At the same time the piston pressurizes the charge in the crankcase. Crank case inlet can be controlled by a reed valve, by the piston skirt, or by a rotating disc valve. As the piston moves down it reveals the exhaust port letting the exhaust exit the cylinder. Moving further down the piston reveals the scavenging port that connects the cylinder above the piston and the crankcase. The pressurized charge flows to the cylinder scavenging the remaining exhaust out. In the meantime the piston reaches BDC and then starts traveling towards TDC. First it closes the intake port then the exhaust port completing the two-stroke gasoline cycle.

The strokes of a typical two-stroke diesel engine:

- Intake-compression.

- Expansion-exhaust.

Today’s typical two-stroke diesel engine uses a combined valve-port control to achieve scavenging. It has an exhaust valve that is usually mechanically operated and an intake port opened and closed by the piston. The two-stroke diesel engine also uses a blower or a turbocharger that feeds pressurized air to the engine. The piston moves 
towards TDC compressing the air in the cylinder. Both the intake port and the exhaust valve are closed. At TDC fuel is injected in the cylinder where it autoignites. The burning gases push the cylinder down providing power. Before BDC the exhaust valve opens followed by the intake port and scavenging takes place. In the meanwhile the piston reaches BDC and returns. First the exhaust valve closes then the intake port closes completing the cycle.

In two stroke gasoline engines the scavenging process is controlled by the piston, revealing and covering the intake and exhaust ports. Two stroke diesels have a combined valve-port control. In four stroke engines, in general, valves are applied to fulfill the same role. A mechanically synchronized timing connection controls the valve operation in four-stroke slider-crank engines where the presence of rotating motion offers the simple solution. In linear engines other techniques must be applied for the lack of rotating motion. Electronic timing control and linear electric actuators were considered as a possible solution for valve actuation in four stroke linear engines [41, 51, 52]. On the other hand, the two-stroke gasoline engine applies the convenient piston controlled ports that are appealing for linear engine application for not requiring added mechanism to control scavenging. 


\subsection{Number of cylinders and configuration}

In conventional engines the linear reciprocating motion of the piston is converted to rotating motion using a slider-crank mechanism. As there is one power stroke in two revolutions in four-stroke engines and one power stroke in every revolution in two-stroke engines, practically, a flywheel is applied on the crankshaft. The flywheel absorbs and stores energy on the power stroke and returns energy and moves the piston during the power consuming strokes, namely the intake, compression, and the exhaust strokes. The flywheel also helps the engine to provide a less pulsating torque, or smooth operation, at the crankshaft. Since the flywheel moves the piston during the power consuming strokes a flywheel is necessary for a single cylinder engine. However, in linear engines the application of a flywheel is not an option due to the lack of rotating motion. Theoretically, the lack of a flywheel could be overcome by applying multiple cylinders on the same crankshaft, or translator, with one cylinder for each stroke at a minimum. A piston, during the expansion stroke, will move the other pistons on their power consuming strokes. Although in rotating engines, adding more cylinders yields smoother operation, meaning less pulsating torque, it is not true in linear engines. On the other hand there is a minimum number of cylinders that yields an operation that does not require the application of an energy accumulator. This number is four cylinders for the four-stroke linear engine and two cylinders for the two-stroke linear engine would be the minimum to maintain operation without requiring an energy source or an energy accumulator. 
The following linear engine configurations are achievable practically:

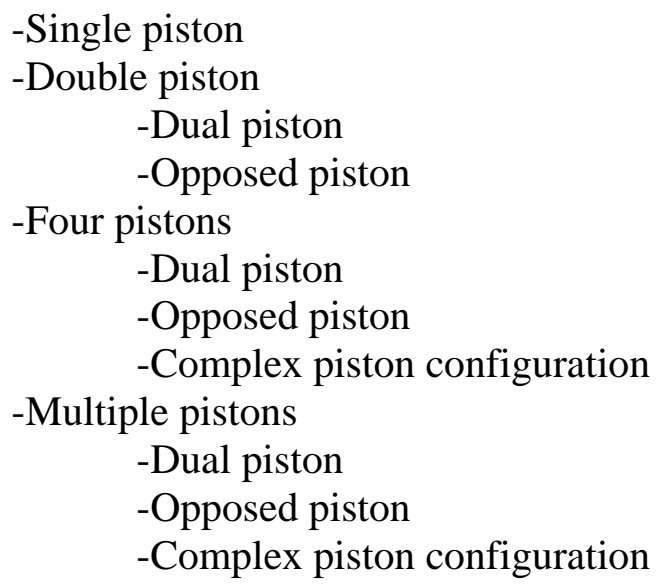

A single cylinder configuration is shown in Figure 25.

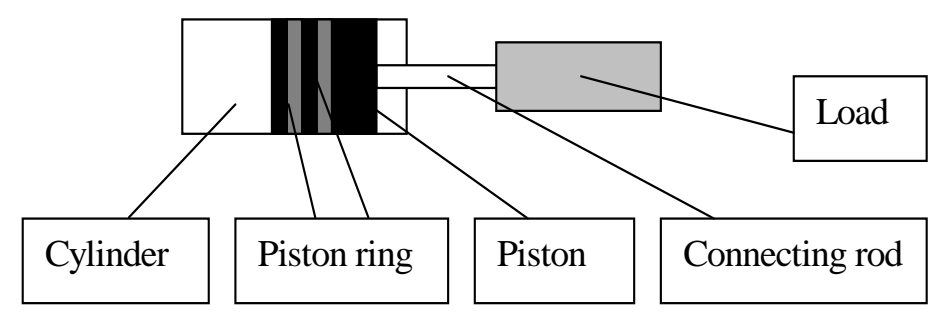

Figure 25. Single piston linear engine configuration.

At a minimum, two pistons must be linked together when using two-stroke engines and four pistons must be linked together when using four-stroke engines to ensure a power stroke for every stroke. An exception is when a double acting piston is used and the volume below the piston as well as the volume above it are both used for combustion. As a conclusion, a power stoke is needed for every half of a frequency cycle (revolution in conventional engines). However, if there are fewer cylinders in a design then an additional power source is required to move the piston(s) during its power consuming stroke(s). A hydraulic accumulator, a linear electric motor, or even a spring can be used to move the piston on the power consuming strokes resulting in continuous operation. Achten [40] presents a working prototype of a two stroke, single cylinder free 
piston engine/hydraulic pump using a hydraulic accumulator that would provide energy during the compression stroke. Prados [41] in his thesis considered a computer simulation for a four-stroke single cylinder linear alternator, in which the alternator would provide the energy to move the piston during the intake, compression, and the exhaust strokes. Prados concluded that such a configuration would not be able to generate net power.

Figure 26 and Figure 27 show the dual piston and the opposed piston arrangements for two cylinder linear engines respectively. Figure 26 shows two arrangements. One is where each piston has its own cylinder and the load is placed between them. The other is when the pistons are united and share a cylinder and load is applied on each side. In the latter one, the combustion takes place on the connecting rod side of the piston, making cylinder sealing a significant issue.

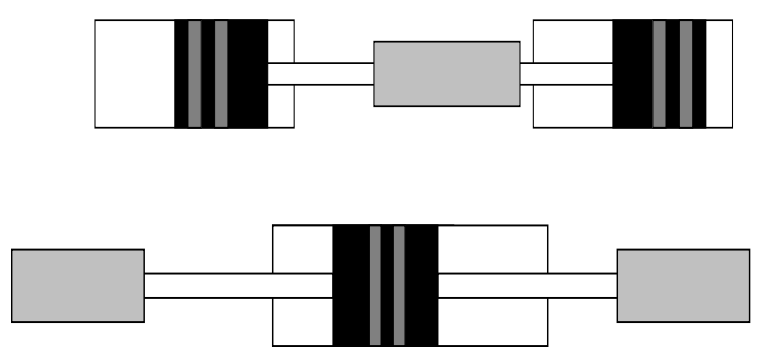

Figure 26. Dual piston linear engine configurations.

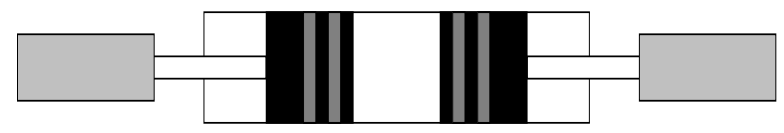

Figure 27. Opposed piston linear engine configuration.

The dual piston version is desirable for two-stroke operation as it provides a power stroke for each stroke of the engine. Two cylinders for the dual piston arrangement two-stroke operation are sufficient to run the engine without an external 
energy source or an energy accumulator. However, the pistons, connecting rod, and the moving components of the load form a high reciprocating mass that causes undesired vibration. Braun [39] presented a linear engine that used a counter weight via a rackpinion-rack mechanism to balance the reciprocating mass and eliminate vibration. Braun did not consider the efficiency loss resulting from the counter weight. In addition, the rotating parts of the auxiliary mechanism defeat the concept of linear engine with no rotating and wearing components. A similar counter-weight could be applied in multiple cylinder designs to decrease vibration. However, the opposed piston configuration would eliminate the vibration problem since the pistons always move in the opposite direction resulting in zero net force on the cylinders and the housing.

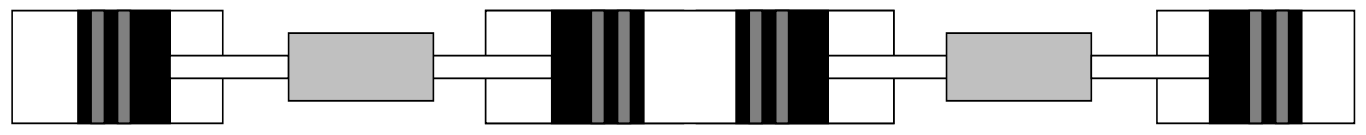

Figure 28. Four-cylinder complex configuration.

Figure 28 shows a four-cylinder arrangement with complex dual and opposed pistons configuration. On the two-stroke cycle this engine will have a power stroke for each cycle and the reversed motion of the two piston-load assemblies will eliminate vibration.

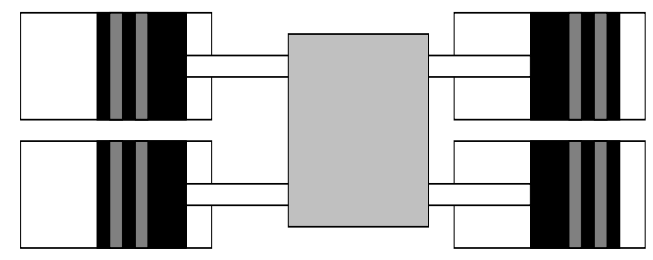

Figure 29. Four-cylinder dual piston linear engine configuration. 


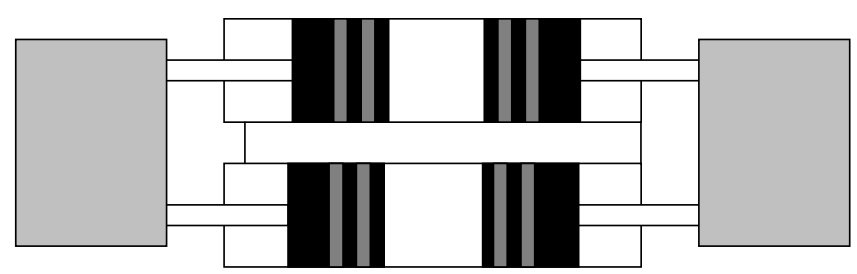

Figure 30. Four-cylinder opposed-piston configuration.

Figure 29 and Figure 30 show the dual and opposed piston arrangements respectively for four-cylinder linear engines. The cylinder that is on the expansion stroke moves the other cylinder(s). This creates a side thrust on the pistons that is undesirable. Although two cylinder designs could be linked together resulting in four, six, eight, or higher number of cylinder configurations, in practice it would be advantageous to have multiple two- or four-cylinder auxiliary power units (APU) on board of a vehicle that can be turned on or off independently from each other when changing power requirement makes it necessary. This is similar to cylinder deactivation that is proposed in large rotational engines with a projected 8-10\% fuel economy improvement [56]. For example a sport utility vehicle (SUV) might have three APUs but it would require only one to be turned on when crawling in bumper to bumper traffic to provide power for moving the vehicle and the load requirement of the power steering, air conditioning, and other comfort and safety equipment in the vehicle. Cruising on a highway at $70 \mathrm{mph}$ might require two APUs to be on, and hauling heavy load would use all the three. In this way the friction and heat losses of the six cylinders would be reduced to that of two or four cylinders during light load conditions. Underwood presented a working prototype twostroke four-cylinder arrangement applied as a gasifier for a gas turbine [57]. He called it a Siamese engine in which the two two-cylinder linear engines share a common housing, intake and exhaust manifolds, and an air box but there is no physical connection between them. The advantage of the Siamese design is while one engine is being scavenged the 
other compresses resulting in better breathing of the engine. Petreanu presented a computer simulation for four-cylinder four-stroke linear engine in his dissertation [59]. He analyzed an arrangement similar to that in Figure 29. In such an engine there would be a force between the cylinder wall and the piston that would result in higher friction eliminating one of the advantages of linear engines. 


\subsection{Simulation of the linear engine}

The computer simulation of linear engines can be performed in three stages: simulation of the engine, simulation of the load, and the simulation of the engine and the machine that represents the load coupled together. A vast majority of the literature discusses the simulation of the linear engine itself $[35,50,51,59,68-70,81,82,84]$ using one or two step simulation as described by Kaario [69] and zero or one dimensional models as described by Larmi et al. [64].

Just a small portion of the literature described a combined load-engine model. Olson [58] integrated the gasifier pump model in his simulation and Bromborsky et al. [77] integrated the linear alternator model in the simulations they performed. It can be concluded that for a complete simulation, it is crucial to investigate the combined engineload system. Only that will yield accurate predictions of the prototype operation.

Shoukry [85] developed a numerical simulation program using the principles of a previous four-stroke compression ignition linear engine model [59], with changes made to make it possible to investigate the two-stroke compression ignition linear engine in his study. To generalize results, the program was altered to provide dimensionless parametric analysis. Dimensionless displacement, mass, and time were defined with respect to effective stroke length, moving assembly mass, and exhaust port pressure immediately after port closure. The program inputs were bore to effective stroke length ratio, equivalence ratio, dimensionless injection position, dimensionless load constant for the alternator, and premixed and diffusive combustion ratios. The outputs of the program were dimensionless frequency, dimensionless indicated power, dimensionless friction force, dimensionless average velocity, dimensionless instantaneous velocity, indicated 
efficiency, compression ratio, dimensionless indicated power/generator mass, dimensionless energy/generator mass, dimensionless indicated power/cylinder volume, and dimensionless energy/generator volume.

\subsection{Applications of linear engines}

There were three main applications of linear engines. They were used as gasifiers along with gas turbines, they were used to drive linear positive displacement hydraulic pumps, or they were used coupled with linear alternators.

\subsubsection{Free piston engine}

Linear engines were often called free piston engines in the literature, although the piston is not free in these applications as its path is eventually restricted by the cylinder heads or the bottom of the cylinder. Firearms, on the other hand, could be regarded as free piston engines for having no physical restraints on the piston (the bullet) after it leaved the chamber except for the target and gravity. Since guns were out of the scope of present study this document called the subject a linear engine. 


\subsubsection{Nail gun}

The Paslode company has developed a nail gun that uses a linear engine to drive nails into wooden surfaces [49]. The gun has a fan in the cylinder to ensure scavenging and proper mixing of air and fuel. The user applies a double triggering mechanism by pressing the gun against the surface then pulling the trigger. Pressing the gun to the surface closes the intake and exhaust ports and injects fuel in the cylinder and pulling the trigger activates the sparkplug. Combustion pressure above the piston drives the nail into the surface and compresses air under the piston. When the user takes the gun away from the surface the mechanism opens the exhaust port letting the combustion gases out. At the same time the compressed air under the piston moves the piston to the upper position and the fan scavenges fresh air in the cylinder setting the nail gun for the next cycle. Paslode's nail gun uses a small fuel tank and a $6 \mathrm{~V}$ rechargeable battery. It can drive in 1200 nails on a tank of fuel and 4000 nails on a fully charged battery. The gun uses 16 gauge 1.25 ” to 2.5 ” long nails [49].

\subsubsection{Gasifier}

Linear engine gasifiers in various sizes and arrangements were designed, simulated, and manufactured by GM, Ford, Renault, Junkers, Sigma, and others [50 - 58]. They all fulfilled the same role: they were combusters for gas turbines.

A gasifier engine pumps clean air and its own exhaust into a tank from where the warm, pressurized mixture of air and exhaust is led to the inlet of the gas turbine. The operating stages of a gasifier are shown in Figure 31. 

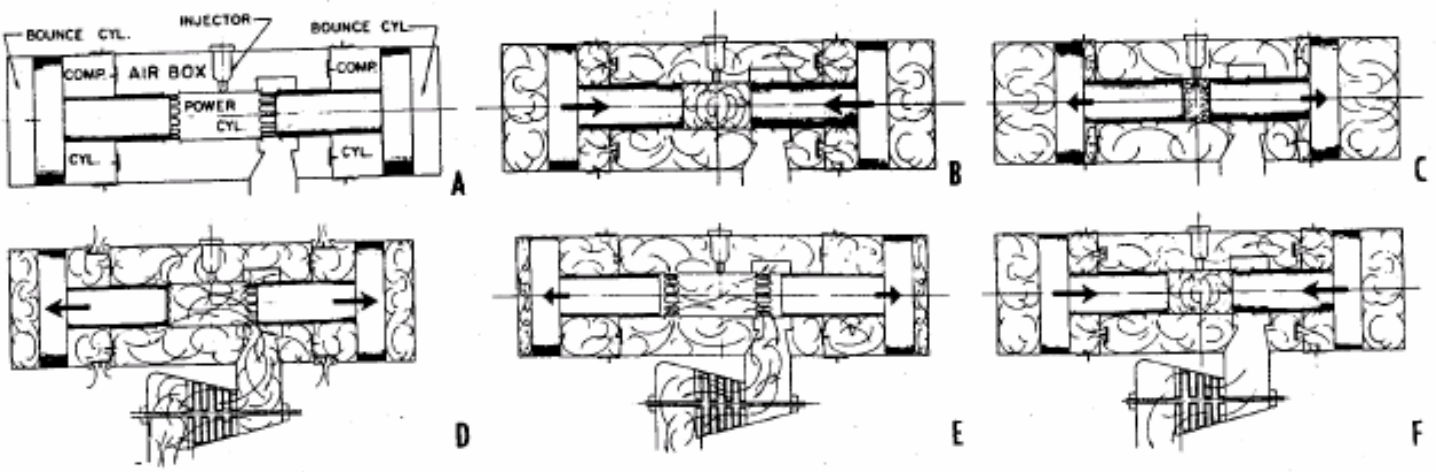

Figure 31. Operating sequence of an opposed piston linear engine gasifier [57].

The particular design shown in Figure 31 is an opposed piston arrangement with a common combustion chamber in the middle and bounce cylinders at the two ends. The bounce cylinders are basically air springs that accumulate energy during expansion and return it during compression. The energy stored in the air springs is used to compress the air in the combustion cylinder during combustion. When proper compression is reached fuel is injected into the combustion chamber where it autoignites and presses the pistons outward compressing air in the bounce chambers and sucking air into the space under the large piston pump. When the ports are revealed exhaust exits the cylinder and fresh air from the air box flows through the cylinder to the air tank. The pistons start moving inward, as the result of decreased pressure in the combustion chamber and the high pressure in the bounce chamber. Compression starts when intake and exhaust ports are covered by the pistons and with the same motion the piston displaces the air from the space under the pump pistons to the pressure box and the cycle starts again. 


\subsubsection{Hydraulic or pneumatic pump}

Linear engines can be connected to positive displacement, linear, hydraulic or pneumatic devices such as piston or membrane pumps to generate pressure or flow supply for hydraulic or pneumatic actuators or motors. Several different designs and arrangements were developed and simulated for various purposes during the past 40 years [39, 40, 60-69]. Amongst them, the dual piston configuration at Tampere University of Technology $[64,65]$ was a typical application and is shown in Figure 32. Tampere University’s design is called Emma2.

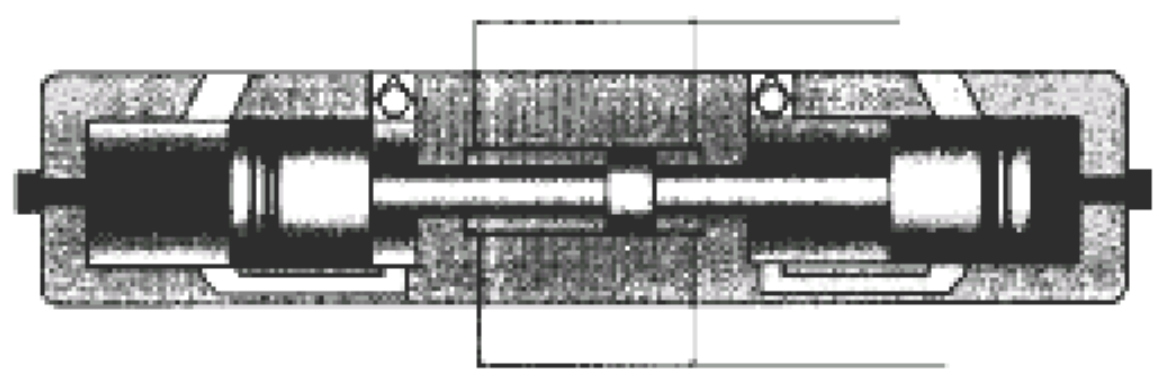

Figure 32. The dual piston hydraulic pump developed at Tampere University of Technology [65].

The Emma2 was a dual-piston, two-stoke, loop scavenged diesel linear engine that drove a hydraulic pump located between the cylinders. As it was a dual piston twostroke design, the Emma2 did not require assistance from the hydraulic pump to sustain operation. The piston diameter was $90 \mathrm{~mm}$ and the stroke was $120 \mathrm{~mm}$. The engine applied a Ganser-Hydromag common rail injection system. Tikkanen et al. [65] reported 11.3 $\mathrm{kW}$ power output at $28 \mathrm{~Hz}$ operating frequency and 16:1 compression ratio. The engine was started using the hydraulic pump as a hydro-motor. Tikkanen et al. reported approximately 20\% total efficiency for the Emma2 hydraulic free piston engine [65]. 


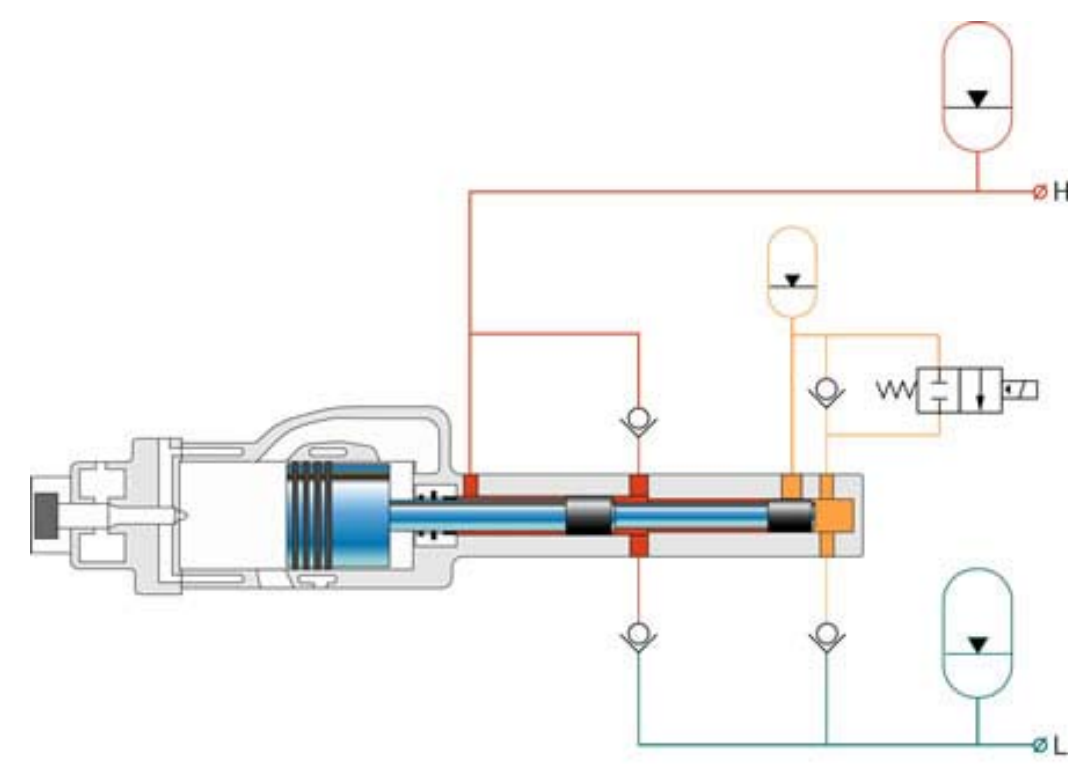

Figure 33. The single piston hydraulic pump developed at Innas B.V. [62].

The single cylinder design at Innas B.V. [40, 60-63] stands out with its originality. Figure 33 shows the "CHIRON" of Innas B. V., a single cylinder linear engine hydraulic pump. As it was a two-stroke single-cylinder design, a hydraulic accumulator was applied to store energy during the power stroke that was used to drive the piston during the compression stroke. The CHIRON used pulse-pause modulation to control the operational frequency. When the piston was on the power stroke, the hydraulic pump used its power to pump the fluid as a useful power output. At the same time, part of the power was used to build up pressure in the compression accumulator through a check valve. Because the CHIRON was a single cylinder engine there was nothing that would push the piston up to cause compression. The system stopped and stayed in a standby state until the frequency control valve opened and let the pressure of the accumulator act on the compression piston that moved the piston towards TDC compressing the air in the cylinder. After compressing the air, fuel was injected and combustion took place resulting in a power cycle, pumping fluid from the low-pressure 
side to the high-pressure side as well as to the hydraulic accumulator. The process was repeated at the opening of the frequency control valve. Achten et al. [61] reported as low operating frequencies as necessary to provide the required flow rate or pressure. Actually the piston moves with similar speeds for different operating frequencies but it spends varying amount of time at BDC waiting for the frequency control valve to open realizing pulse-pause modulation.

The CHIRON had a stroke of $120-125 \mathrm{~mm}$ and a piston diameter of $110 \mathrm{~mm}$. Its net effective power was $17 \mathrm{~kW}$ with up to 51\% indicated efficiency. As Achten et al. concluded efficiency and emissions could be controlled by injection timing and the pressure in the compression accumulator [65]. The authors reported insignificant increase in exhaust emissions due to the cold start of the engine. They did not report on efficiency loss due to heat loss to the cold cylinder walls.

\subsubsection{Electric generator}

A linear engine can be coupled with a linear generator to form an electric generator set. The generator set can be used as a stand-alone mobile power generator or as an auxiliary power unit of a hybrid vehicle. Several research groups have performed conceptual analysis, simulation, and prototype design of the linear engine-alternator [59, 70-84]. The efforts of two scientist groups are shown here: Sandia National Laboratories

[71-74] and West Virginia University [77-83]. The prototype "RCEM" (rapid compression-expansion machine) of Sandia National Laboratories can be seen in Figure 34. 


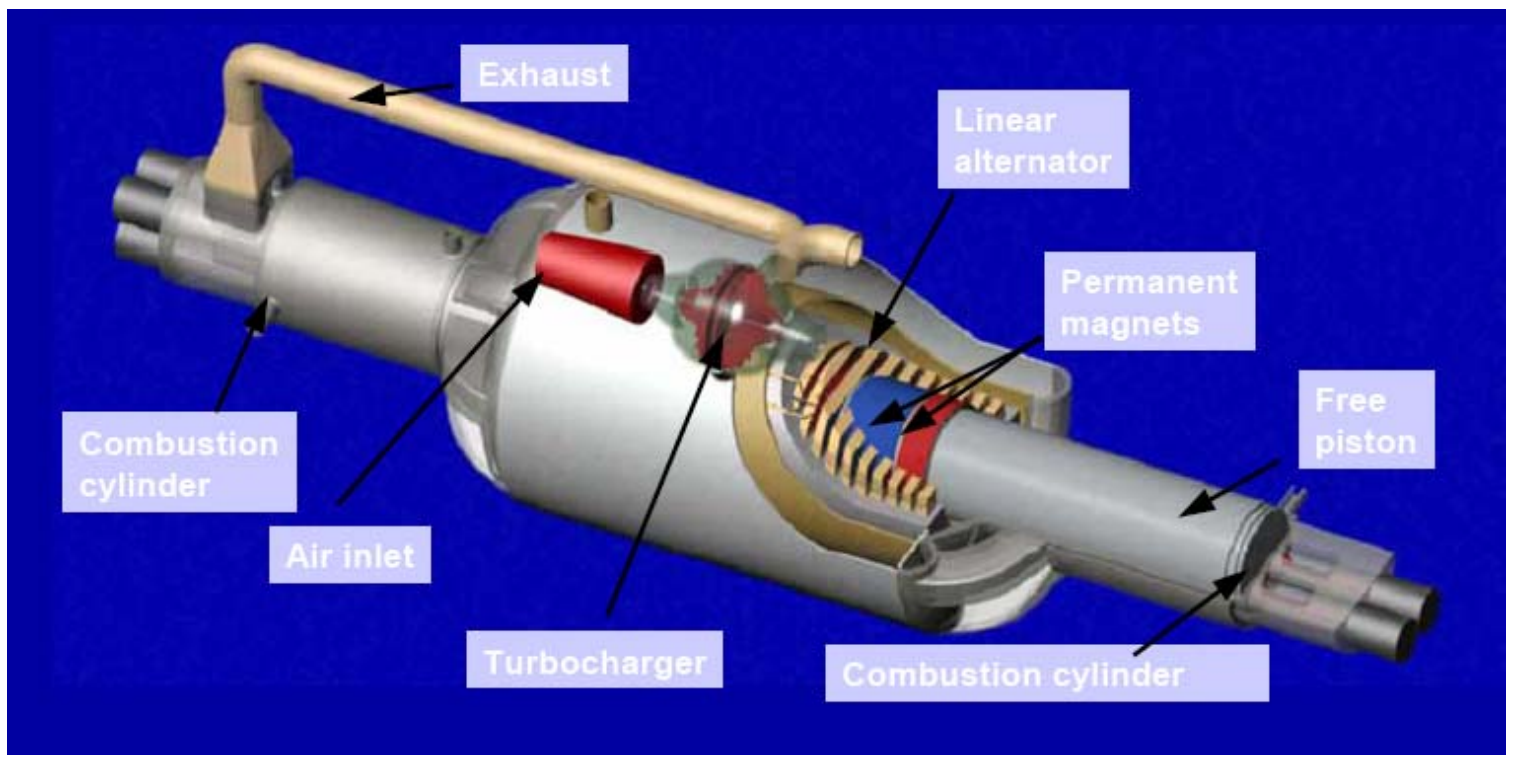

Figure 34. The homogeneous-charge compression-ignition linear engine-alternator developed at Sandia National Laboratories [73].

The linear engine developed at SNL had a dual piston arrangement and operated on a two-stroke cycle therefore the generator never had to work as a motor except for starting the engine. The RCEM was a homogeneously charged compression ignition two-stroke experimental linear engine. It operated at low equivalence ratio (0.3) and at high compression ratios (30:1-70:1). The homogeneous charge and the compression ignition allowed nearly constant volume burning in the cylinder, as a result, it let the engine achieve close to ideal Otto cycle efficiency. Van Blarigan [71] claimed that 95\% of the fuel was burned in $80 \mu$ sec. This resulted in $48 \%$ thermal efficiency as the lowest value for the tested fuels in their experimental engine. Van Blarigan et al. [74] tested the experimental engine on eight different fuels and found as high as $56 \%$ efficiencies for propane and natural gas. The RCEM had a $76.2 \mathrm{~mm}$ bore and $254 \mathrm{~mm}$ maximum stroke. Researchers at SNL have not reported continuous operation. The prototype machine was tested on a single stroke at a time with homogeneous charge compressed to auto ignition in one cylinder with the aid of compressed helium providing the compressing force in the 
other. The linear alternator was used to control compression ratio for the study. The unit was estimated to provide around a maximum of $30 \mathrm{~kW}$ output power at an oscillating frequency of $40 \mathrm{~Hz}$ [71].

Previous research at WVU included the simulation of a four-cylinder four-stroke linear engine [59], the simulation of a two-cylinder two-stroke linear engine [78, 81, 83, 84, 85], and the development of a spark ignited two-cylinder two-stoke prototype linear engine [82]. The linear engine prototype developed at West Virginia University can be seen in Figure 35.

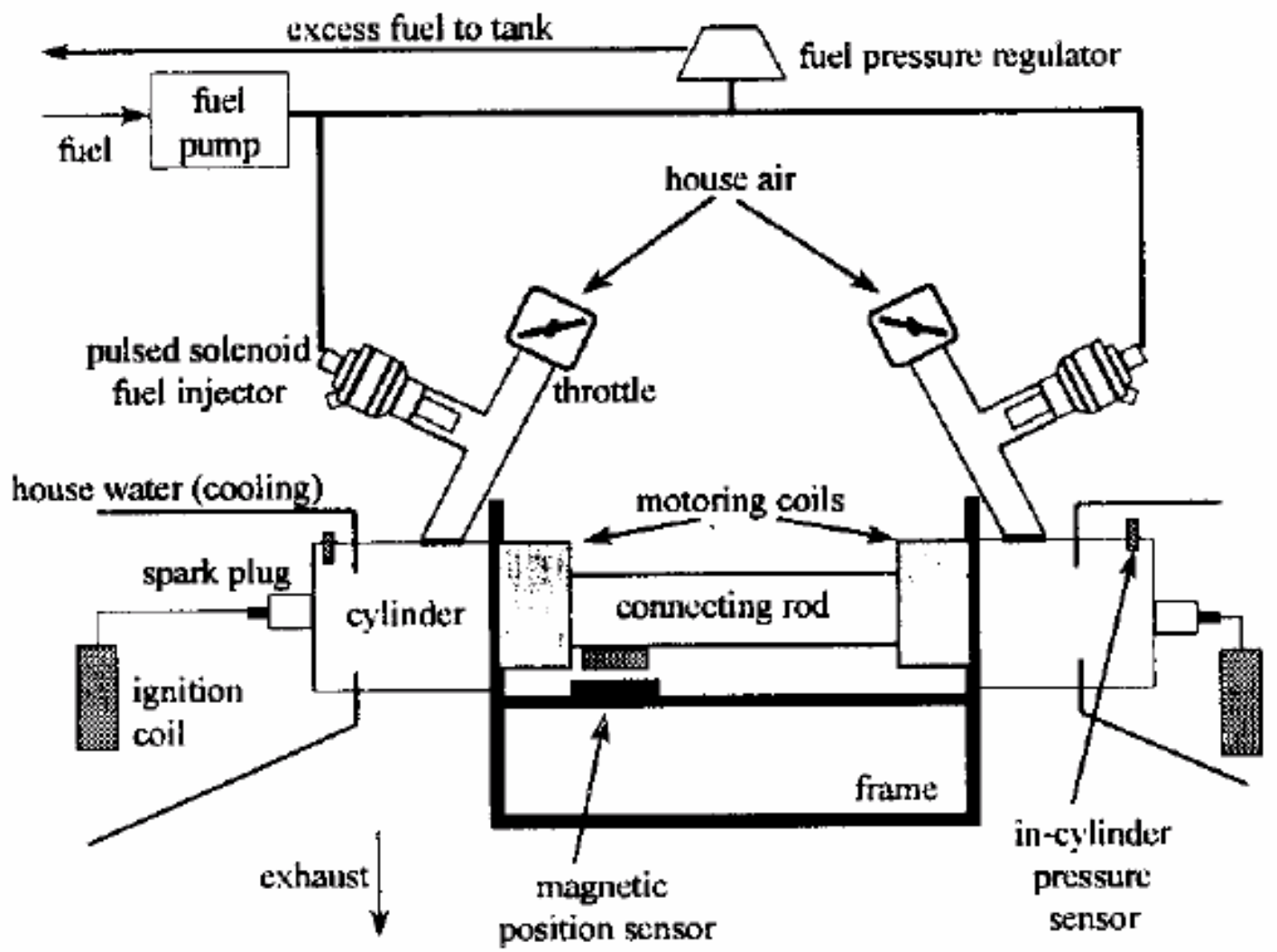

Figure 35. The linear engine developed at West Virginia University, $1^{\text {st }}$ generation [82].

WVU's $1^{\text {st }}$ generation linear engine was also a dual-piston two-stroke model, similar to that of SNL. The main difference lied in the fact that WVU's linear engine was 
spark ignited. The engine had $36.5 \mathrm{~mm}$ bore and a maximum stroke of $50 \mathrm{~mm}$. Clark et al. [82] reported sustained operation under idling conditions as well as under load. A brushless permanent magnet DC alternator was used to apply load and gain power from the system. Load was reportedly increased to $316 \mathrm{~W}$ before the engine stalled. The authors concluded that the presented linear engine was far from being optimized so efficiency was not reported. Another suggestion of the study was that throttled operation is undesired for the pumping losses that the throttle represents. This called for a diesel version of the shown design.

\subsection{Conclusions}

This chapter summarized the present state of research and development of linear engines. After the linear engine was introduced, possible variations were presented such as internal or external combustion, two-stroke or four-stroke, operation based on Diesel or Otto cycle. Various piston and cylinder arrangements were also shown resulting in a high number of possible configurations of linear engines. Furthermore, linear engine applications under development were briefly presented.

It was shown that an unthrottled operation would result in higher efficiency than that of throttled. It was also shown that a homogeneous charge compression ignition version of the linear engine could get close to Otto cycle efficiency. Although HCCI requires a complicated control and allows limited load and operating speed variations in conventional engines, it is suited for linear engine applications because the engine can achieve virtually any compression ratio. It was also shown that linear engines were less expensive to manufacture and to maintain than slider crank engines [39]. Thus it can be concluded that an unthrottled HCCI engine would provide an efficiency increase and cost 
reduction compared to conventional engines. It was also shown that a four cylinder combined dual-opposed piston arrangement would not require an external energy source or the application of an energy accumulator taking advantage of the dual piston arrangement. At the same time the opposed arrangement would eliminate the vibration problem that is inherent in the dual piston configuration. Based on these facts it can be concluded that the linear engine represents a promising platform as a high efficiency power source and an excellent candidate for auxiliary power unit in series hybrid electric vehicles.

Although several scientific groups performed simulations on the linear engine, only a few of them have realized a working prototype. This shows the possible complexity in control of a mechanically simple system and the fact that the linear engine still needs extensive development before it could be commercially available. 


\section{$4 \quad$ Theoretical analysis}

This chapter presents a dynamic and thermodynamic analysis of the linear engine followed by a dimensional analysis and the computer simulation model of the engine.

\subsection{Analysis of the linear engine}

In a linear engine, there is no crankshaft that would define the piston's motion or its top and bottom dead center. Instead, the piston speed and position is the result of the forces acting on the translator and top and bottom dead centers can vary from cycle to cycle. A linear engine with dual piston arrangement was analyzed for the present study.

The alternating mass consists of the pistons, piston rings, piston pins, pin retainers, connecting rod, the moving components of the electric machine, and the fastener components. The forces acting on the translator are: inertial force, friction force, gas forces (compression and expansion), and load force. The linear engine can be thought of as a simple mass-spring system as shown in Figure 36. Figure 37 shows the free body diagram of the translator.

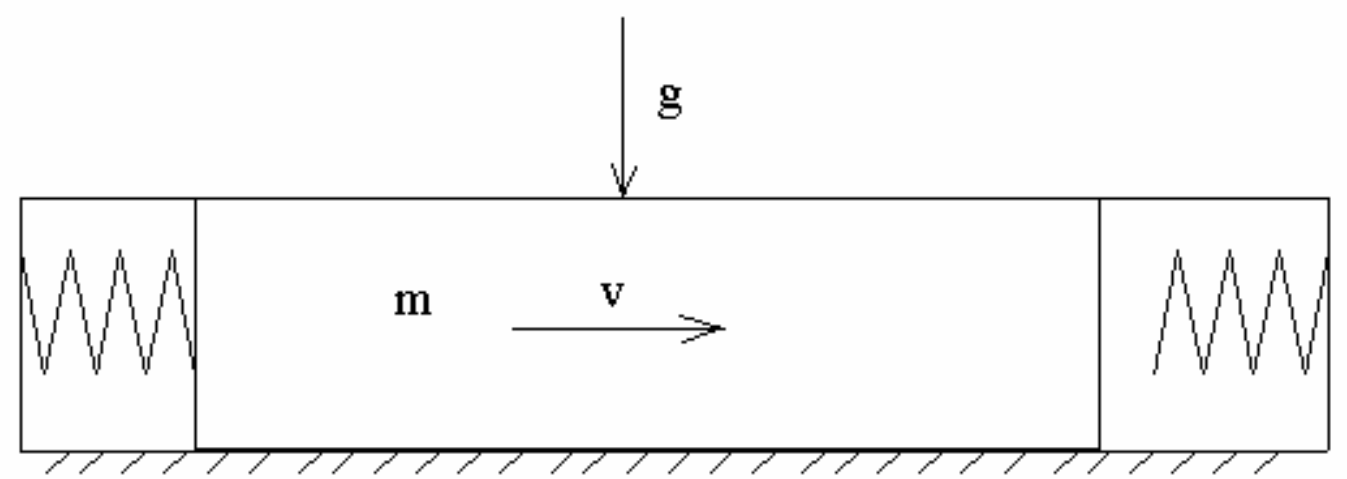

Figure 36. The linear engine can be thought of a mass spring system. The forces acting on the alternating mass (translator) define its motion. 
Figure 37 shows the free body diagram of the linear engine translator being driven by expanding combustion gases.

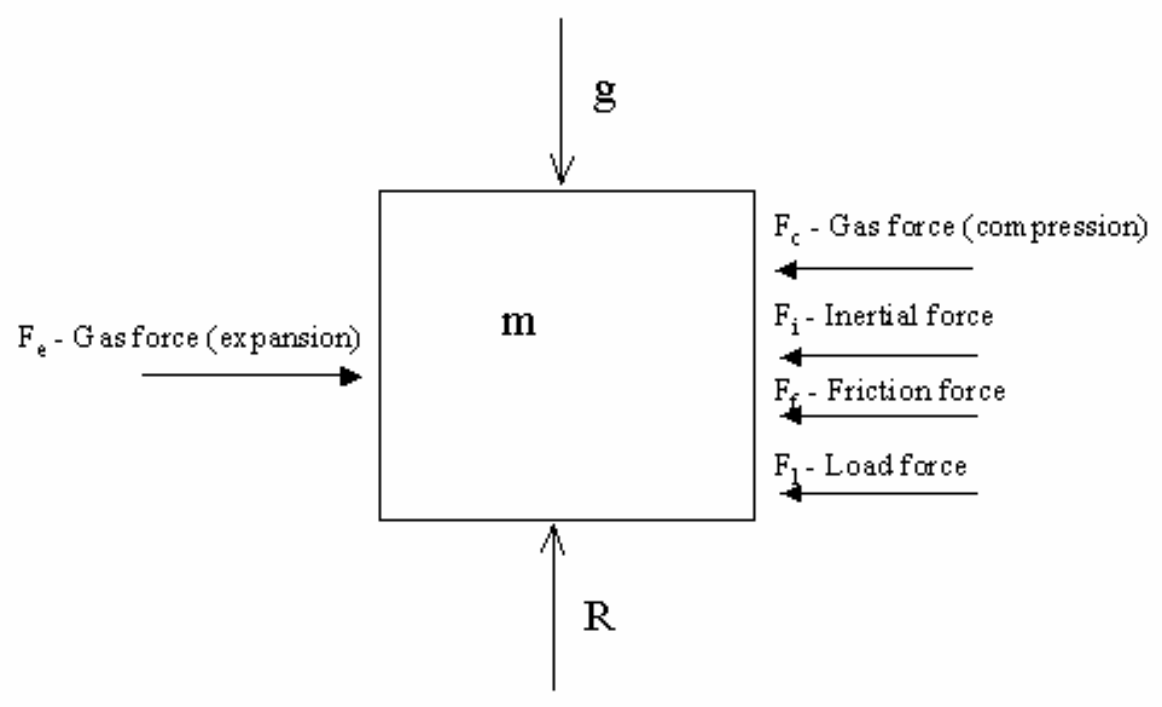

Figure 37. The free body diagram of the linear engine. Gravity is represented by $g$ and the reaction force is represented by $R$.

The mass represents the combined mass of the translator, the two springs represent the gas being compressed in the cylinders and the exciting force represents the gas forces during expansion or compression. The gap between the translator and the spring on the right hand side represents the range where ports are open in both cylinders at the same time. The exhaust port is already uncovered in the expansion cylinder, letting the expansion pressure blow-down. At the same time, ports are still uncovered in the compression cylinder therefore compression has not started yet. Only inertial, load, and friction forces act on the mass at this time. This is the free travel distance. As the force equilibrium defines it, the sum of the forces acting on the mass must be equal to zero.

\section{Equation 1.}

$$
\Sigma F=0=F_{i}+F_{f}+F_{l}+F_{c}-F_{e}
$$




\subsubsection{Inertial force}

Newton's second law of motion defines the inertial force based on the mass and the acceleration of the translator:

\section{Equation 2.}

$$
F_{i}=\boldsymbol{m a}
$$

\subsubsection{Friction force}

A large amount of literature discusses the problem of friction in internal combustion engines in conventional rotating engines. Scientists have developed simplified friction models to simulate engine friction [110-113]. In a linear engine, the situation is already simplified compared to a rotating engine, because there is no crankshaft. Except for the effect of gravity (which is negligible compared to the side thrust in a slider-crank engine), there is no side thrust on the piston, therefore the piston does not move in the cylinder in radial direction. The sources of friction force in a linear engine are friction between the piston rings and the cylinder liner and friction between the piston skirt and the cylinder liner. A simple approach was chosen to model friction force in the simulation. Equation 3. shows the equation that was used to calculate friction force in the simulation. $V_{p}$ represents the velocity of the translator and $f_{d}$ represents the friction coefficient.

\section{Equation 3.}

$$
F_{f}=f_{d} V_{p} \quad f_{d}=f(\text { bore, translator mass) }
$$

The constant $f_{d}$ was the friction coefficient. It was represented as the function of bore size and translator mass. These two parameters were chosen because the size of the bore influences the friction caused by ring tension and the mass influences the friction 
caused by gravity. A simple trial and error approach was applied to adjust and find the friction coefficient for the simulation model to match experimental operation. For further details see Chapter 8.

\subsubsection{Gas forces}

Two types of gas forces act on the piston-load assembly compression and expansion. Because the engine operates on a two-stroke cycle, compression takes place in one cylinder while expansion takes place in the other and vice versa. Because the engine is symmetrical, the modeled processes taking place in the two cylinders are identical, happening with a half cycle time shift between them. The gas force $\left(\mathrm{F}_{\mathrm{g}}\right)$ can be calculated from the gas pressure $\left(\mathrm{p}_{\mathrm{g}}\right)$ multiplied by the area of the piston's bottom $(\mathrm{A})$ as Equation 4 shows it. Pressure was calculated from the heat release equation.

\section{Equation 4.}

$$
F_{g}=p_{g} A
$$

\subsubsection{Heat release}

Heywood [91] described the combustion process in an internal combustion engine as a single zone model with the first law of thermodynamics as shown in Equation 5.

\section{Equation 5.}

$$
\frac{d Q_{c h}}{d t}=\frac{\gamma}{\gamma-1} p \frac{d V}{d t}+\frac{1}{\gamma-1} V \frac{d p}{d t}
$$

The first law of thermodynamics does not include the effects of heat transfer to the cylinder walls, flow into and out of crevices, and leakage. Heywood [91] described the "heat release" approach that incorporated the change in sensible energy $\left(d U_{s}\right)$, 
chemical energy released during combustion $\left(\delta Q_{c h}\right)$, the work on the piston $(\delta W)$, the heat transfer to the cylinder walls $\left(\delta \mathrm{Q}_{\mathrm{ht}}\right)$, and the crevice flows $\left(\mathrm{hdm}_{\mathrm{cr}}\right)$. Heywood used a simple single zone model. The model assumed the cylinder contents as a single zone. Equation 6 describes their relationship.

\section{Equation 6.}

$\delta Q_{c h}=d U_{s}+\delta W+\delta Q_{h t}+\Sigma h_{i} d m_{i}$

The accuracy of the model depends on how accurately each term in Equation 6 can be defined. Equation 7 through Equation 9 show the expansion of each term in Equation 6.

\section{Equation 7.}

$d U_{s}=m c_{v}(T) d T+u(T) d m$

\section{Equation 8.}

$$
\delta Q_{h t}=A h_{c}\left(T-T_{w}\right)
$$

\section{Equation 9.}

$\delta W=p d V$

Substituting Equation 7 through Equation 9 into Equation 6 yields Equation 10.

\section{Equation 10.}

$\delta Q_{c h}=m c_{v} d T+\left(h^{\prime}-u\right) d m_{c r}+p d V+A h_{c}\left(T-T_{w}\right)$

Applying the ideal gas law neglecting the change in Re and $c_{v}$ results:

\section{Equation 11.}

$$
\delta Q_{c h}=\left(\frac{c_{v}}{\operatorname{Re}}\right) V d p+\left(\frac{c_{v}}{\operatorname{Re}}+1\right) p d V+\left(h^{\prime}-u+c_{v} T\right) d m_{c r}+\delta Q_{h t}
$$

Inserting $\gamma=\mathrm{a}+\mathrm{bT}$ (the way $\gamma$ changes as a function of temperature) into the equation yields: 


\section{Equation 12.}

$\frac{d Q_{c h}}{d t}=\frac{\gamma}{\gamma-1} p \frac{d V}{d t}+\frac{1}{\gamma-1} V \frac{d p}{d t}+V_{c r}\left[\frac{T}{T}+\frac{T}{T_{w}(\gamma-1)}+\frac{1}{b T_{w}} \ln \left(\frac{\gamma-1}{\gamma^{\prime}-1}\right)\right] \frac{d p}{d t}+\frac{d Q_{h t}}{d t}$

For the present study, the flow in to and out of crevices was neglected thus the equation simplified to Equation 13.

Equation 13

$\frac{d Q_{c h}}{d t}=\frac{\gamma}{\gamma-1} p \frac{d V}{d t}+\frac{1}{\gamma-1} V \frac{d p}{d t}+\frac{d Q_{h t}}{d t}$

From Equation 13, $\frac{d p}{d t}$ can be calculated for force analysis purposes.

\subsubsection{Scavenging air pressure}

Scavenging is the process of the fresh charge replacing the burned gases in the cylinder. Scavenging in a conventional two-stroke engine can be modeled as the combination of the two extreme scavenging models: perfect displacement and complete mixing [91]. Since the air was supplied from a pressurized external source with a high flow rate scavenging did not take any energy from the engine in the case of the prototype linear engine. For the simulation, a simple charge displacement model was used. It was assumed that scavenging air completely replaced the burned gases in the cylinder for positive values of free travel. For negative values of free travel, the gas replacement was modeled as a linear function of free travel with $100 \%$ replacement of burned gases at free travel $=0$ and $0 \%$ replacement at free travel $=$-effective stroke. This model was used to compensate for the fact that with increasing negative free travel, the revealed crossection of the scavenging port was decreased. In the cylinders of the prototype linear engine loop scavenging took place. Intake air was introduced into the space under the 
piston (crank case in a conventional engine) and the piston itself controlled the scavenging port. The scavenging air pressure was present on the bottom of the piston unless the scavenging port was uncovered. In this particular case, the scavenging air helped compressing the charge in the cylinder and worked against the gas forces during expansion; however, this effect was negligible.

\subsubsection{Load force}

At this stage of the prototype development, a friction brake provided the load for the linear engine. Equation 14 calculates the force (F) on a conductor of a given length (l) moving with a velocity (v) in a magnetic field (B). Equation 15 calculates the force (F) caused by friction $\left(f_{d}\right)$ that moves with a velocity $(v)$.

\section{Equation 14}

$$
F=B l v
$$

\section{Equation 15}

$$
F=f_{d} v
$$

In both equations the force is a function of velocity. It can be concluded that the application of a friction brake to mimic the load created by the alternator is a valid approximation.

The friction brake was clamped on the shaft of the linear engine. It was cable operated and a load cell measured the tension in the cable. The load cell was calibrated to provide the axial force on the shaft (the load on the engine) to the data acquisition program. After the engine was started and fueling was set, the load was gradually applied until the engine stalled. Power output was calculated from the operational 
frequency right before the engine stalled and the applied force. The same method was applied in the simulation to define the maximum power output that the engine can provide.

\subsection{Dimensional analysis}

Dimensional analysis was performed on the linear engine for two reasons. Firstly, to decrease the number of variables involved in the experiments with the prototype linear engine. The number of variables was decreased from eleven to eight, of which five were independent variables and three were dependent. Secondly, to establish a scale for the similitude concept so the experiments done on the existing prototype linear engine could be used to predict results for linear engines of various sizes and dimensions. Buckingham's Pi theory [117] was used to reduce the number of variables from eleven to eight.

The variables involved with the linear engine were defined based on the force analysis in Section 4.1. The inertial force involved the mass of the translator. The gas forces involved cylinder geometry (bore and stroke), fuel amount injected, and the pressure of the scavenging air. The load force was simply friction force for the present study. Another important variable was injection position. Normal (close to TDC) injection would result in DICI operation but early injection was expected to result in a pseudo-HCCI operation. Pseudo-HCCI occurs when fuel is injected early (around BDC). After the fuel is injected in the cylinder, it would mix with air and autoignite as soon as charge temperature reaches the ignition temperature of the fuel. Note that the critical cylinder conditions might be achieved by different compression ratios for different 
charge compositions and ambient conditions. This would result in varying stroke and varying top and bottom dead center positions.

The variables involved are listed below with their SI units.

- Bore

- Stroke (effective)

- Stroke (total)

- Mass of translator

- Mass of fuel injected

- Load

- Injection position

- Scavenging air pressure $[\mathrm{m}]$

$[\mathrm{m}]$

[m]

[kg]

[kg]

$\left[\mathrm{N}=\frac{\mathrm{kgm}}{\mathrm{s}^{2}}\right]$

[m]

$\left[\frac{k g}{s^{2} m}\right]$

The effective stroke is the distance between the upper edge of the exhaust port and the cylinder head. The total stroke is the distance that the translator can travel from cylinder head to cylinder head.

The dependent variables were:

- Operating frequency

- Compression ratio

- Efficiency $\left[\frac{1}{s}\right]$

$\left[\frac{V}{V}\right]$ Dimensionless

$\left[\frac{J}{W s}\right]$ Dimensionless

The reference variables were chosen so that they would be independent from eachother:

o Bore

o Mass of translator

o Scavenging air pressure $[\mathrm{m}]$

[kg]

$\left[\frac{k g}{s^{2} m}\right]$ 
Dividing all the variables by the reference variables results the dimensionless variables:

- $\quad$ Effective stroke/ Bore

- Total stroke/ Bore

- $\quad$ Mass of fuel injected/ Mass of translator

- $\quad$ Load/Scavenging air pressure/Bore ${ }^{2}$

- $\quad$ Injection position/Bore

- Operating frequency/(Scavenging air pressure) ${ }^{1 / 2}$ (Mass of translator) $^{1 / 2} /$ Bore $^{1 / 2}$

- $\quad$ Compression ratio

- $\quad$ Efficiency $=$ Dimensionless Operating frequency

= Dimensionless Effective stroke

$=$ Dimensionless Total stroke

$=$ Dimensionless Fuel mass

= Dimensionless Load force

= Dimensionless Injection timing

$=$ Dimensionless Compression ratio

= Dimensionless Efficiency

\subsection{Conclusions}

The linear engine could be modeled as a simple mass, spring, and damper system. Forces acting on the mass were inertial force, friction force, gas forces, and load force. The role of these forces was shown in this chapter. A dimensional analysis was also carried out with the intension of using the experimental data gained in this study to predict the behavior of different sized linear engines. However, the database was not sufficient for that purpose and further investigation is needed to fill that gap. 


\section{Computer simulation}

Computer simulation and results of WVU's second-generation linear engine were presented by Shoukry et al. [84] and in the dissertation of Shoukry [85]. The simulation used dynamic and thermodynamic numerical equations to analyze the engine’s behavior. The authors used a time-based model for the simulation. Parameters modeled included rate of combustion, heat transfer to the cylinder walls, friction, piston position, speed and acceleration, in-cylinder pressure and temperature, and load. Shoukry et al. [84] used a single zone model considering a homogeneous ideal gas charge in the cylinder. The model did not take into account the presence of vaporizing liquid droplets, fluid flow, combustion chamber geometry, or spatial variations of the mixture and temperature. The study used dimensionless terms to generalize the simulation results. Results showed the effect of changing reciprocating mass, injection timing, injection duration, and load on piston position and velocity, cylinder pressure and temperature, indicated efficiency, and operating frequency.

From the paper by Shoukry et al. [84] it can be concluded that early injection results in loss of efficiency. Shoukry et al. concluded that this might be due to the fact that early combustion wasted energy to stop the piston when the piston still has high kinetic energy.

A simulation model was created and simulation was performed for the present study. The goal of the simulation was to generalize the experimental results and to predict the behavior of different size linear engines. A point of interest was to find the 
combination of component sizes that yielded the highest mechanical efficiency for the linear engine.

The simulation was performed in Matlab [90] using dynamic and thermodynamic numerical equations. The simulation was computationally expensive. Running a 10 second long run took over an hour of computation. To decrease simulation time, an artificial neural network was trained to predict engine behavior. An initial set of 50 simulation runs was created using the simulation. The engine parameters were randomly chosen and simulation was run on the data set. Then the neural network was trained. The training process took about 20 seconds and the prediction with the neural network took less that a second. A genetic algorithm was used to find the combination of component sizes that yielded the best efficiency. It was clear that the neural network was not accurately trained on a limited number of simulation runs, therefore the point that the genetic algorithm had found to be the best was entered into the simulation model and simulation was performed. The result of the simulation was added to the data set that was used again to train the neural network. This improved the accuracy of the prediction. This process was applied recursively until the efficiency predicted by the genetic algorithm and the neural network was equal to the efficiency simulated by the model.

\subsection{Simulation model}

Further simulation was needed for a better understanding of the processes in the linear engine. For this particular study, a simple simulation model was constructed to predict the behavior specifically for the prototype linear engine (described in Chapter 6). The simulation used Newton's second law of motion with inertial force, friction force, 
gas forces on the pistons, and load force to calculate the acceleration of the translator. Numerical integration yielded translator velocity and position.

The simulation model did not account for fuel vaporizing effects, fluid flow, swirl in the cylinder, or cylinder geometry. The model was created with the assumption that the cylinder content was a homogeneous charge of ideal gas. The actual simulation code can be viewed in Appendix C. Results of the simulation are shown in Chapter 8.

\subsubsection{Gas forces}

The cylinder content was assumed to be a single zone of ideal gas. Pressure in the cylinder was calculated using the first law of thermodynamics in the form of Equation 16.

\section{Equation 16.}

$$
\frac{d p}{d t}=-\gamma \frac{p}{V} \frac{d V}{d t}+(\gamma-1) \frac{1}{V} \frac{d Q}{d t}
$$

Heat transfer was accounted for, using a simple heat conduction model, which assumed the inside of the cylinder wall to be gas temperature and the outside to be coolant temperature. Heat radiation and convection were neglected. Gas temperature was calculated by the ideal gas law shown in Equation 17.

\section{Equation 17.}

$$
T=\frac{p V}{m R_{\text {air }}}
$$

\subsubsection{Heat release}

The heat release rate was calculated using double Wiebe functions described by Shoukry et al. [84]. Equation 18 shows the formula that was used for calculating the heat release rate. 


\section{Equation 18.}

$$
\frac{d Q}{d t}=a \frac{Q_{p}}{t_{p}}\left(M_{p}+1\right)\left(\frac{t}{t_{p}}\right)^{M_{p}} \exp \left(-a\left(\frac{t}{t_{p}}\right)^{M_{p}+1}\right)+a \frac{Q_{d}}{t_{d}}\left(M_{d}+1\right)\left(\frac{t}{t_{d}}\right)^{M_{d}} \exp \left(-a\left(\frac{t}{t_{d}}\right)^{M_{d}+1}\right)
$$

Shoukry [85] recommended the values $\mathrm{M}_{\mathrm{p}}=0.5, \mathrm{M}_{\mathrm{d}}=3$, and $\mathrm{a}=1.2$ so they were used in the present study. The other constants were chosen $t_{p}=150 \mu$ s (premix burning time), $t_{d}=5$ ms (diffusion burning time) to result in a burning time usual in a diesel engine.

\subsubsection{Friction force}

Friction force was accounted for with a damping term in the simulation. The damping coefficient for the simulation model was determined by trial and error by visually matching simulated engine operation with engine behavior. The model behavior matched the engine behavior well. Correlation coefficient for the simulated and measured position was 0.995 . Figure 38 and Figure 39 show the ringing down portion of a test run with no load on it for both simulated and experimental data. 


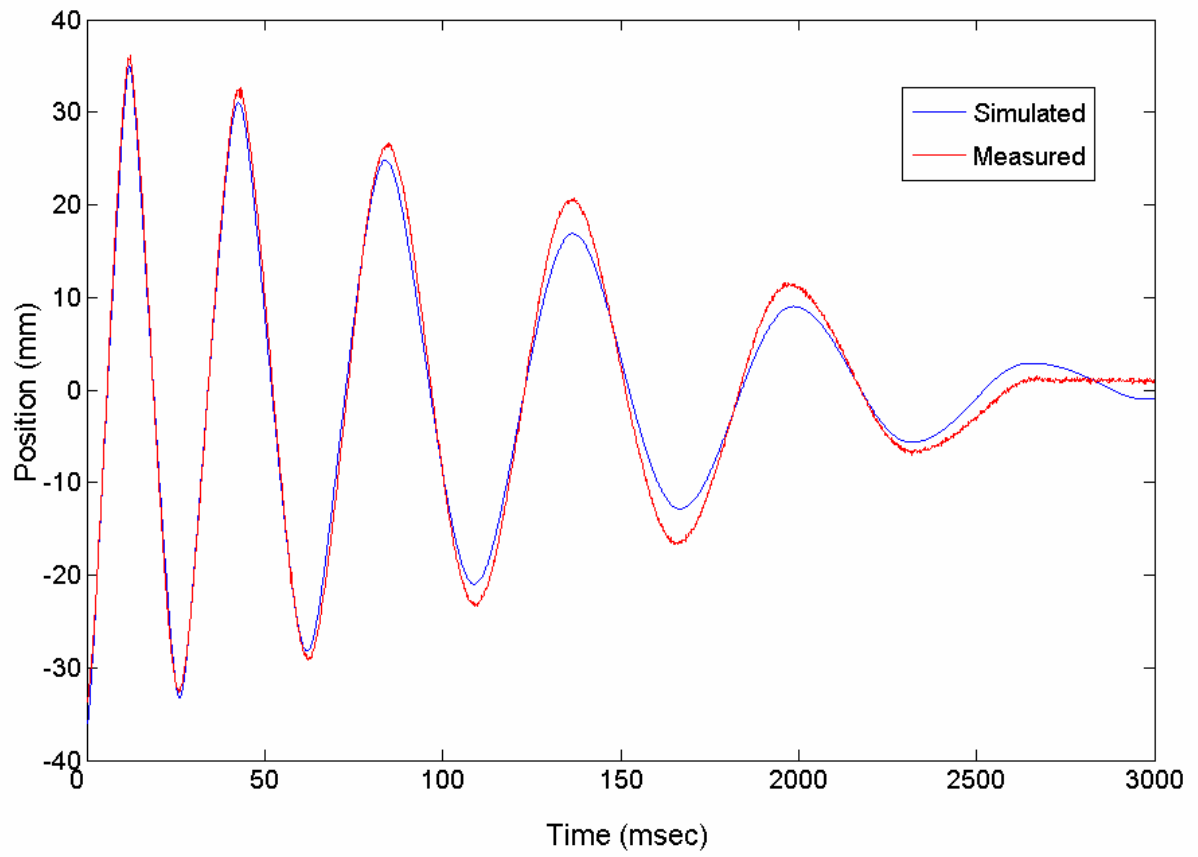

Figure 38. Simulated and experimental translator position after engine was turned off by cutting the fuel. (This phenomena is referred to ringing down).

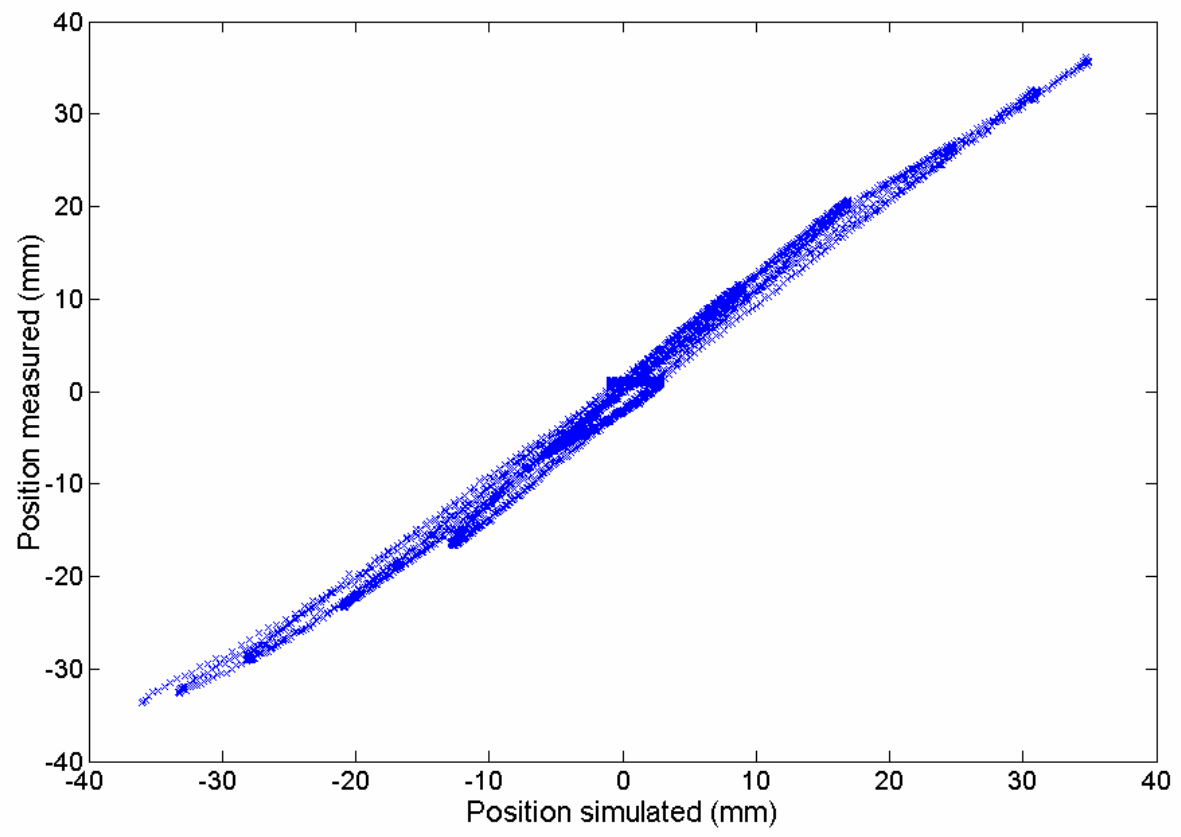

Figure 39. Simulated vs. experimental translator position after engine was turned off (ringing down). Correlation coefficient: 0.995 . 


\subsection{The predictor/simulator model}

A combination of an artificial neural network model and a genetic algorithm along with the simulation model was used to find the engine parameter set that provided the highest efficiency. Figure 40 shows the flow chart of the predictive simulation technique that was used to find the set of engine parameters that had the highest efficiency.

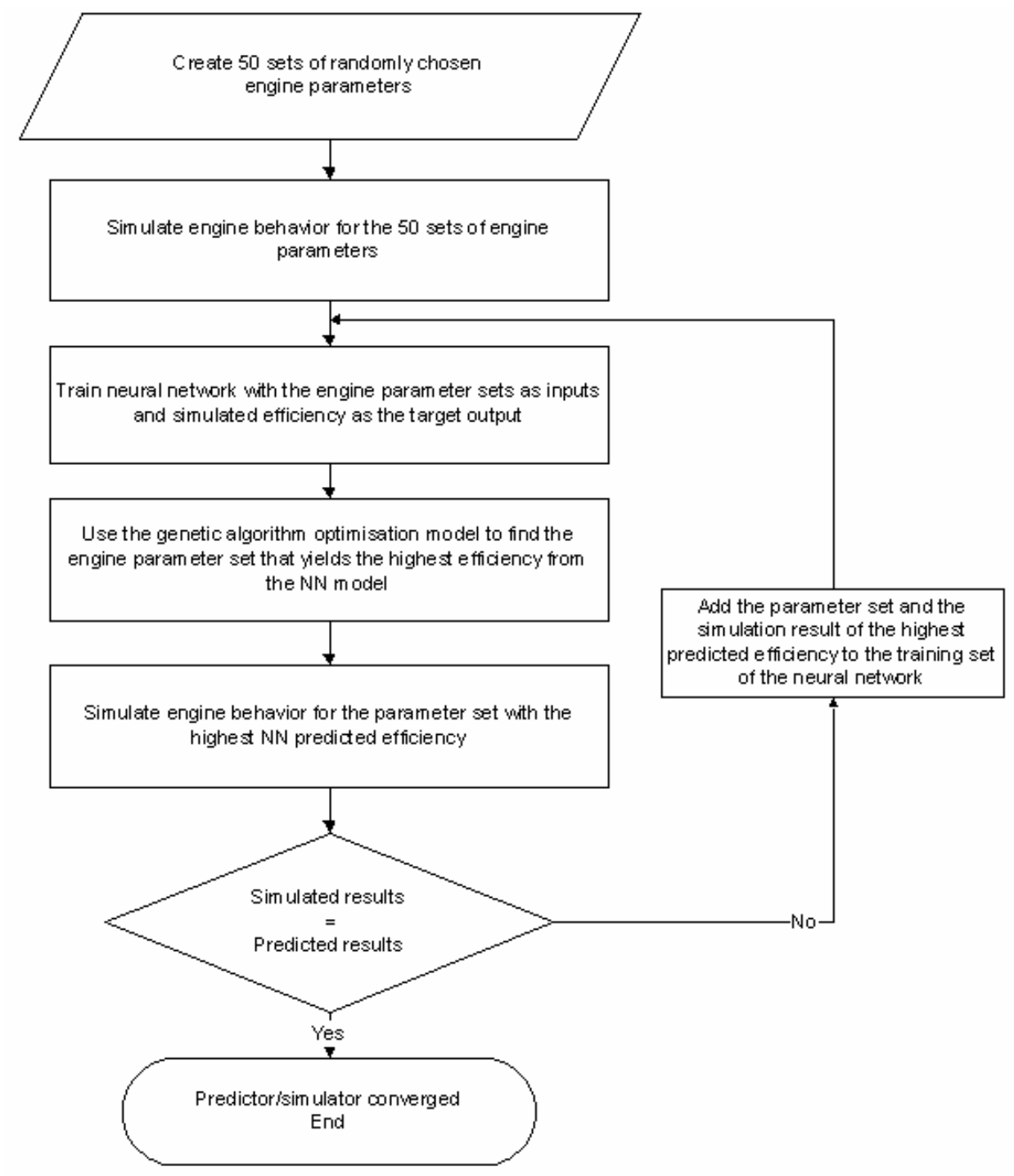

Figure 40. The flow diagram of the predictor/simulator algorithm used to find the engine with the highest efficiency. 


\subsubsection{Artificial neural network (ANN) model}

Artificial neural networks were invented while searching how the human brain works. The learning mechanism of an ANN imitates the learning mechanism of the brain. Artificial neural networks have been used to control and/or to predict in many different applications including engine and emissions research [86-89]. Because neural networks were showed to be a powerful tool to predict nonlinear relationships, an ANN architecture was chosen to predict engine efficiency in this study. The neural network was trained on simulation data. The ANN was used to replace the simulation model to decrease computation time. In this study, MATLAB's neural network toolbox [90] was used. Figure 41 shows the actual neural network architecture. It was a feed-forward back-propagation network. The seven input parameters were bore, stroke, free travel, mass of translator, amount of fuel injected, injection timing, ignition timing and the output parameter was engine efficiency. The inputs were normalized between -1 and +1 before being presented to the neural network. The hidden layer consisted of 25 neurons and the activation functions were tangent sigmoid.

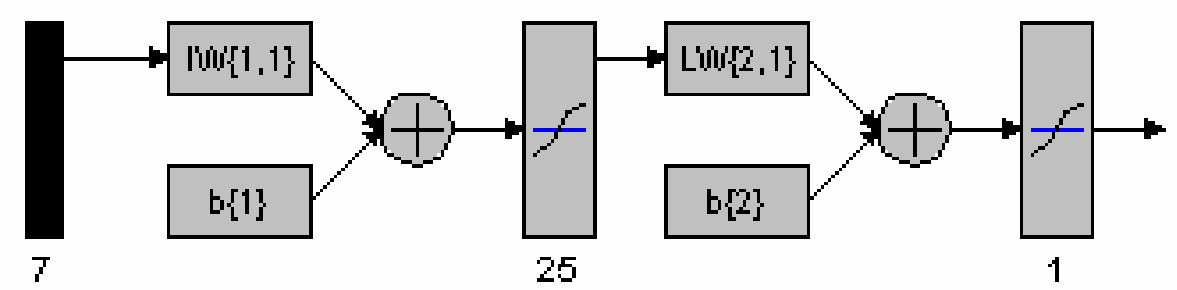

Figure 41. The neural network architecture used for predicting engine efficiency based on bore, stroke, free travel, mass of translator, amount of fuel injected, injection timing, ignition timing. IW and $L W$ indicate the weights in the input and the hidden layer respectively. $b(1)$ and $b(2)$ indicate the thresholds in the input and the hidden layer respectively. 
The neural network was used in a two separate modes. In mode one, the training mode, the neural network was initialized with random weights and thresholds then presented with the training data set and the desired output data set. In several iterations of batch training, the neural network learned (by adjusting the weights and thresholds) the relationship between the inputs and the desired output. In mode two, the predicting mode, once the neural network was trained, it was presented with the input data from the genetic algorithm and predicted the engine efficiency. The neural network prediction was used to replace the simulation model to decrease the computational time.

\subsubsection{The genetic algorithm (GA)}

Genetic algorithms imitate the evolution of genes [108]. They are a powerful tool for optimization problems. A genetic algorithm was used in this study, to find the combination of engine parameters that yielded the highest mechanical efficiency.

\subsubsection{The initial gene pool}

As the first step, the engine parameters were coded into 10 digit binary codes and stringed together to form a chromosome. Engine parameters included bore, effective stroke, free travel, translator mass, amount of fuel injected, injection timing, and ignition timing. Each chromosome was a 70 digits long binary code and represented the seven engine parameters. An original gene pool of 50 chromosomes was created by randomly choosing engine parameters. The parameters were stored in binary form to prevent the accumulation of round off error that occurred when data were converted from binary form to decimal form and vice versa or normalized and scaled back to unnormalized form for the neural network use. The parameters were translated back to decimal code and the 
simulation model was run on each set of parameters to obtain the simulated efficiencies. The neural network was trained on the initial gene pool using the engine parameters as the input data and the simulated efficiencies as the desired output.

\subsubsection{Survival of the fittest / multiplication}

The survival of the fittest function worked in two steps. In the first step, the GA chose the chromosome that resulted in the highest efficiency and replaced the five lowest efficiency chromosomes with it. Then it made the new chromosomes propagate to the top of the gene pool. In the second step, the GA found the second, third, fourth ... sixth best efficiency chromosomes and the worse six chromosomes and replaced the six worst with the six best. Then it propagated the new chromosomes to the top of the gene pool right behind the best chromosomes in decreasing order. This yielded the survival and the multiplication of the fittest.

\subsubsection{Crossover}

The genetic algorithm kept the first two chromosomes unchanged and crossed the other ones. In every even numbered chromosome the GA crossed a randomly chosen binary string with a string in the same position from the chromosome above it. This generated a new pool of genes.

\subsubsection{Mutation}

The GA kept the first chromosome unchanged and mutated the rest of the gene pool. The best chromosome on the top stayed unchanged. The mutation process randomly flipped the binary bits in the chromosomes. The mutation rate was $3 \%$. 


\subsubsection{Neural network prediction/Genetic algorithm}

The new gene pool was presented to the trained ANN and the neural network predicted the efficiency for each chromosome.

The genetic algorithm was run again on the new gene pool based on the predicted efficiency values. The GA with the ANN was iteratively applied until the best chromosome surfaced. This chromosome yielded the best mechanical efficiency only for the given neural network model.

\subsubsection{Testing the genetic algorithm}

The engine parameters formed a seven dimensional variable space that made the testing of the genetic algorithm difficult. Therefore the GA was tested on twodimensional functions. Equation 19 and Equation 20 show the two functions that were used to test the genetic algorithm. The GA was used to find the global maximum of the functions.

Equation 19

$$
z=3(1-x)^{2} e^{\left(-x^{2}-(y+1)^{2}\right)}-10\left(\frac{x}{5}-x^{3}-y^{5}\right) e^{\left(-x^{2}-y^{2}\right)}-\frac{1}{3} e^{\left(-(x+1)^{2}-y^{2}\right)}
$$

\section{Equation 20}

$$
z=\sin 2 x-\sin 3 y+(x+y) 0.03
$$

The genetic algorithm always converged for the function shown in Equation 19 since the local and global maximums were substantially different. This was not the case for Equation 20. Equation 20 resulted in 5 local maximums that hardly differed from the global maximum. However, the genetic algorithm found the global maximum in about $60 \%$ of the attempts. In the case of the other $40 \%$ it settled on one of the local 
maximums. This was not a problem in this application because the neural network had to be retrained and the GA had to be reapplied to the data set several times. The iterations of the GA testing can be found in Appendix A.

\subsubsection{Recursion of the neural network training}

The ANN was trained on a limited number of data sets therefore its prediction capabilities were also limited. This was the reason for the iterative retraining of the ANN. The chromosome that was found to be the best by the GA was decoded and the decoded engine data was presented to the simulation model that simulated the engine efficiency. This new set of engine parameters, along with the simulated efficiency, was added to the original neural network training set and the ANN was retrained. Then the neural network was applied to the engine data stored in the new set of chromosomes and engine efficiencies were predicted again. The genetic algorithm was run again on the original gene pool extended with the new gene. This process was applied iteratively until the neural network predicted efficiency and the simulated efficiency were equal. Results are presented in Section 8.6.

\subsection{Conclusions}

A simple simulation model was developed to verify that the trends of the experimental data and to gain extra insight into the behavior of the linear engine. The simulation model used dynamic and thermodynamic equations to simulate engine behavior, and Wiebe functions to simulate heat release rate. The model did not account for fuel vaporizing effects, fluid flow, swirl in the cylinder, or cylinder geometry. The model was created with the assumption that the cylinder intake was a homogeneous 
charge of ideal gas at the point of port closure. The model was verified with the ringing down portion of a test run and showed a correlation coefficient of 0.995 .

A combined neural network / genetic algorithm routine, which also incorporated the simulation model, was created to find the set of engine parameters that yielded the highest efficiency. The recursive routine was tested on two different two-dimensional functions and was found appropriate for the purpose of finding the linear engine with the highest efficiency. 


\section{Prototype design}

WVU’s second-generation linear engine design incorporated a linear engine and a linear alternator to form an electrical generator set that could be used as an auxiliary power unit of a hybrid vehicle or as a mobile generator set. Figure 42 shows the major components of the linear engine.

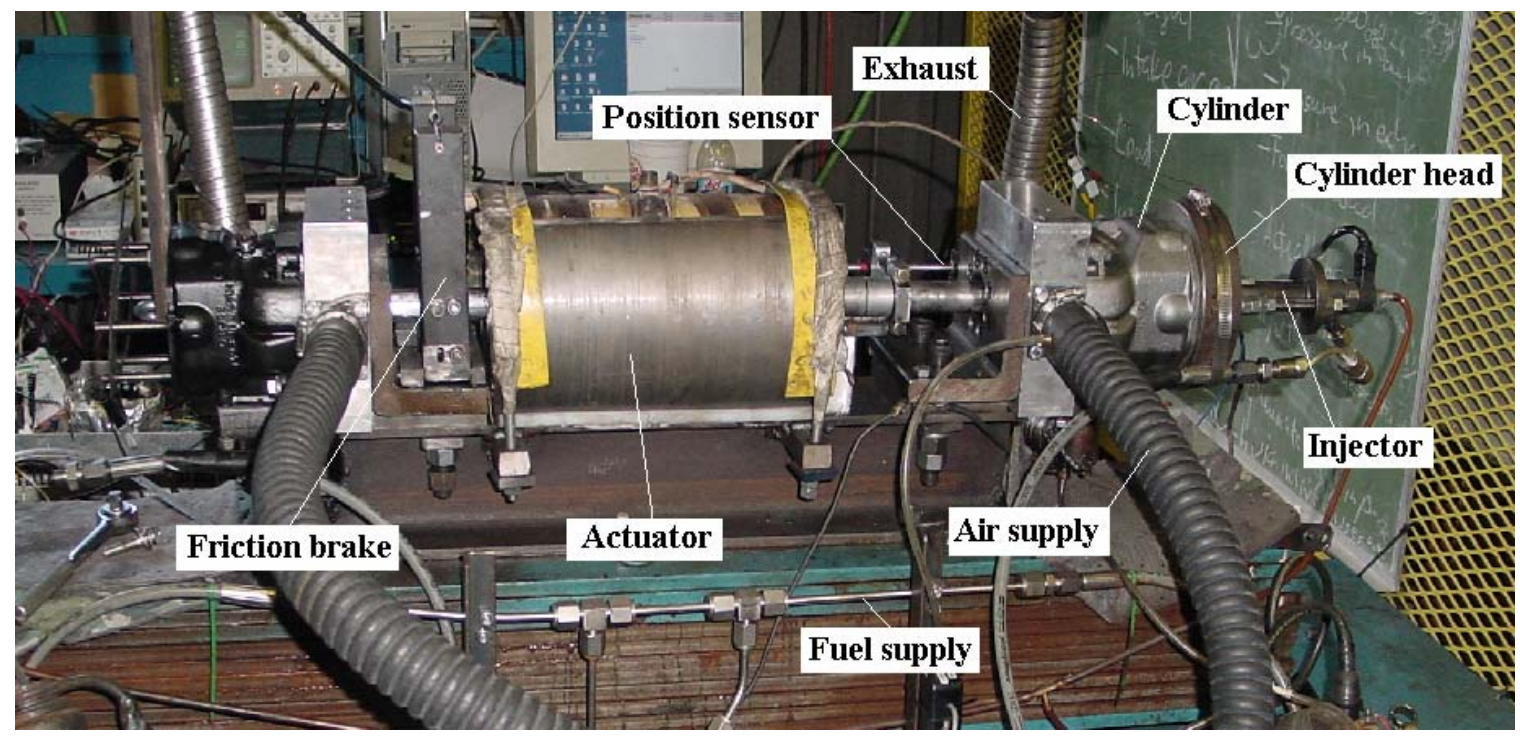

Figure 42. The second-generation linear engine at WVU.

Figure 42 shows the linear engine in a semi-disassembled state. The cylinder head on the left was removed for modifications.

\subsection{Linear alternator}

At the time of the experiments the alternator was still under development. The alternator was required to operate in two modes, namely alternator mode and actuator mode. When in actuator mode the linear alternator would provide the cranking force to start the engine. Once the engine is started the alternator would operate in alternator mode to generate electricity. There were two possibilities considered: permanent magnet 
linear alternator (PMLA), and electro-magnet linear alternator (EMLA). Of the two possibilities, the permanent magnet linear alternator would provide a higher power density with a less complicated control. Its disadvantage would be that the magnetic field on the translator is not controllable. This might result in undesired voltage spikes when switching from actuator mode to alternator mode. Compared to the permanent magnet linear alternator, the EMLA has several advantages as described by Bromborsky [77]. Its major advantage is that the field flux of the machine is controllable, allowing active regulation of the output voltage. This design allows for easy starting of the engine by controllable electromagnetic force when the alternator operates as an actuator. Disadvantages of the EMLA include the need for connection to an external excitation source and heating effect of the current flowing in the winding. The heating can limit the current density of the field windings, resulting in decreased strength of field. Another disadvantage of the EMLA is the increased weight to magnetic field strength ratio of the moving mass because of the amount of copper required to provide a magnetic field that is comparable to the PMLA.

\subsection{Linear engine}

The engine is a dual-piston arrangement, two-stoke, compression-ignition linear engine. Figure 43 shows the arrangement of the second-generation linear engine developed at West Virginia University. 


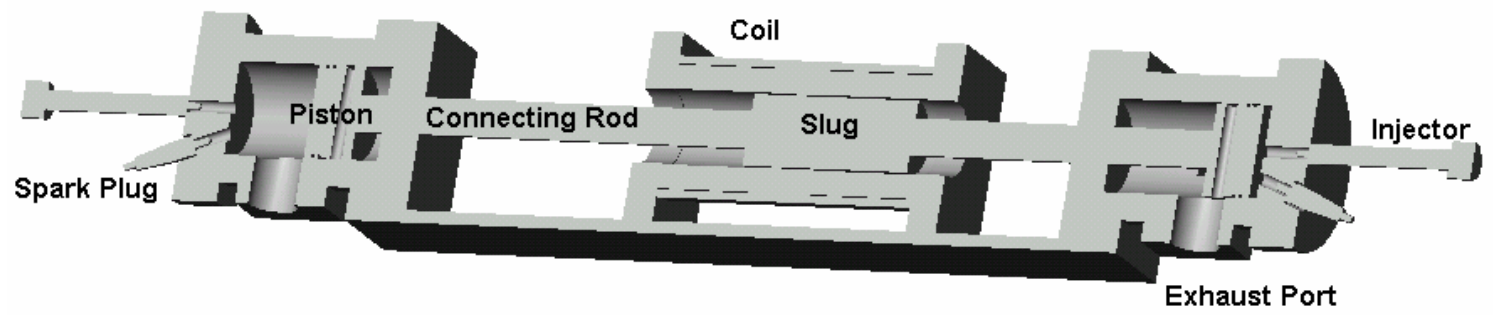

Figure 43. The linear engine developed at West Virginia University [109].

The engine was designed and built by McDaniel [118]. His intention was to build the linear engine from off-the-shelf components to minimize design and manufacturing cost and time. The design was based on Kawasaki cylinders and pistons out of a watercraft engine (JS300). The cylinders were two-stroke, gasoline with a bore of $76 \mathrm{~mm}$ (part \#: 11005-3714). The effective stroke of the engine was $38 \mathrm{~mm}$ and a full stroke 76 mm. The effective stroke indicates the distance between the cylinder head and the upper edge of the exhaust port and the full stroke represents the distance that the piston can physically travel. The cylinders were arranged on a common axis about $800 \mathrm{~mm}$ apart. The pistons were connected with a $25.4 \mathrm{~mm}$ diameter aluminum shaft that accommodated an iron slug in the place of the magnet of the linear alternator. Scavenging air was supplied to the bottom side of the piston from a $83 \mathrm{kPa}$ pressure source. The air flowed to the topside of the piston through the scavenging ports, which were controlled by the piston itself, just as it would occur in a regular two-stroke gasoline engine.

The engine used a Bosch common rail direct injection system with a highpressure pump (part \#: B 445110 031-01) and Bosch injectors (part \#: A611 070 0687). Mercedes-Benz uses these components in many of their diesel engines.

The engine was constructed of off-the-shelf components when it was possible and the rest of the engine was custom made to match the existing components. Each cylinder 
head required an injector, a piezo-electric pressure sensor, manufactured by PCB (part \#: PCB JP 145 A01 SN 212), and a sparkplug (part \#: Champion C59A). A racing ignition system, manufactured by MSD (part \#: MSD6A6200), was used to supply the spark. Ignition was used as an aid to start the engine. The reasons for this are explained in section 6.4

\subsection{Control of the engine}

The linear engine was digitally controlled with a $40 \mathrm{MHz}$ micro-controller (part \#: PIC18F452). The injector control was comprised of a MICRO-EPSILON inductive position sensor (part \# VIP-100-ZA-2-SR-1), the micro-controller, two ignition drivers, and two signal shaping, high-pressure injector drivers [119]. The input parameters to the controller were:

-Position of the translator

-Injection position

-Ignition position

-Reference mean (for calibration)

-Fueling rate

The output signals were:

-Injection on, high current

-Injection on, low current

-Ignition timing

Based on piston position, reference mean, injection position, spark position, and fueling the controller calculated injection timing and duration and ignition timing. A potentiometer was installed to calibrate the reference mean of the system. This was necessary because the engine was disassembled and reassembled frequently, and the location of different components (cylinders, position sensor, wiper of the sensor) had 
slight variation after every time the engine was put together. The reference mean potentiometer was used to compensate for the possible changes in the position sensor calibration. Figure 44 shows the flowchart of the linear engine control.

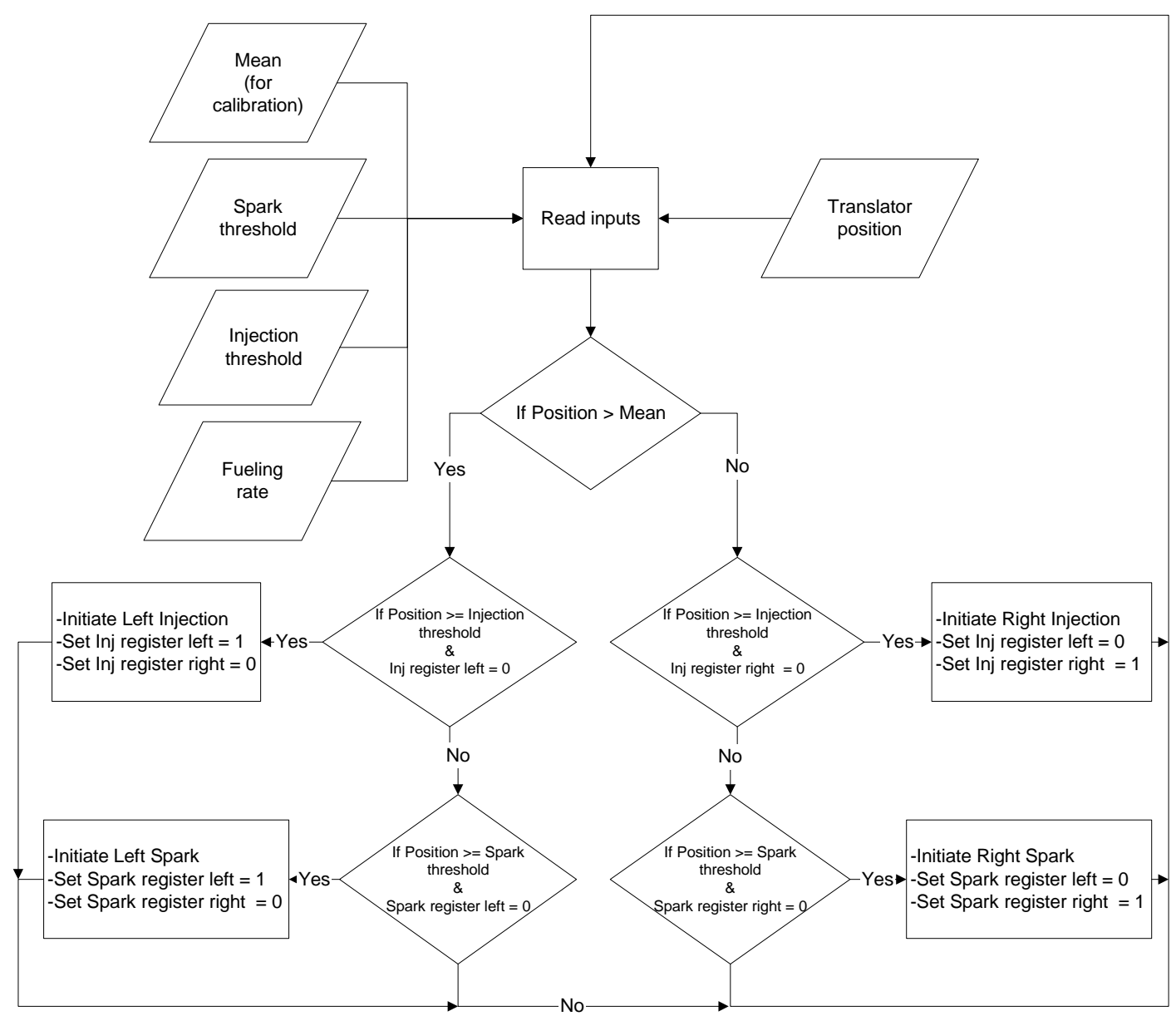

Figure 44. Flowchart of the linear engine control. Translator position was a feedback input. Mean, Spark threshold, injection threshold, and fueling rate were user defined inputs.

Since the injector solenoids required a higher opening current (20 A) for fast action and lower holding current (12 A) for protection of the injector a simple signalshaping circuit was applied to the output of the micro-controller to send the proper current through the injector solenoids. Figure 45 shows controller operation during testing with an input signal from a signal generator. 


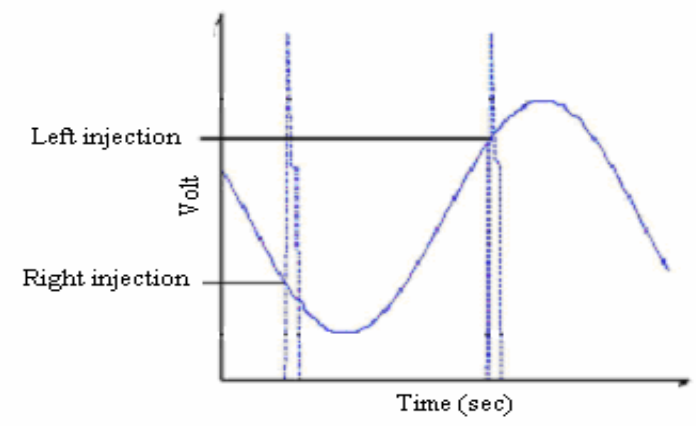

Figure 45. Signal generator signal as input to the controller and the shaped injector signals as response of the controller during controller testing [119].

Although the control program worked with the input signal from the signal generator, noisy input signal from the position sensor resulted in multiple injections. Application of filters was not possible because the time delay they introduced was too long to control the engine at 50-60 Hz of operation. Applying a smoothing function in the code was also not an option because it slowed down the program and introduced a delay. The application of registers in the program code yielded proper injection control even with the noisy input signal. This introduced an error in the precision of injection timing. Figure 46 shows actual injector solenoid control signal during linear engine operation using the position sensor signal as the control input. Noise is clearly visible on the position signal.

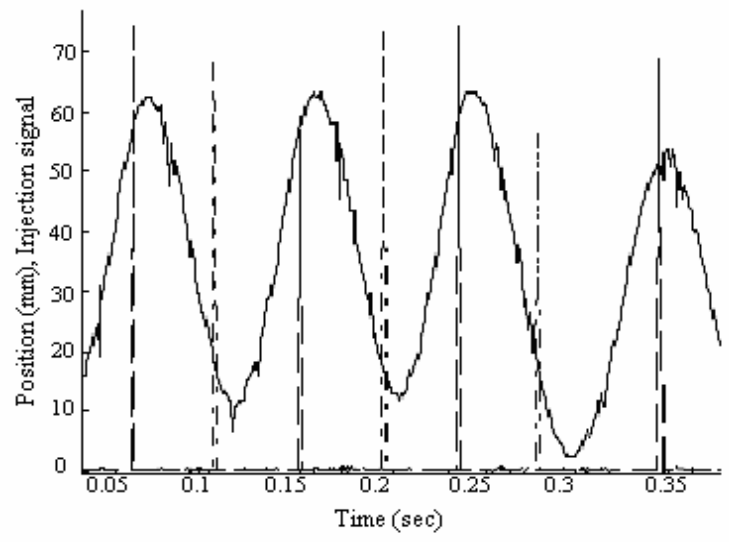

Figure 46. Position of the piston assembly and injection signals vs. time during linear engine testing. The controller provided adequate injection [119]. 
Figure 47 shows the control box of the linear engine. Figure 47 shows the panels that accommodated the IGBT drivers, micro-controller, injector drivers, and ignition drivers. The potentiometers in the bottom right corner are mean for calibration, injection position, spark position, and fueling rate.

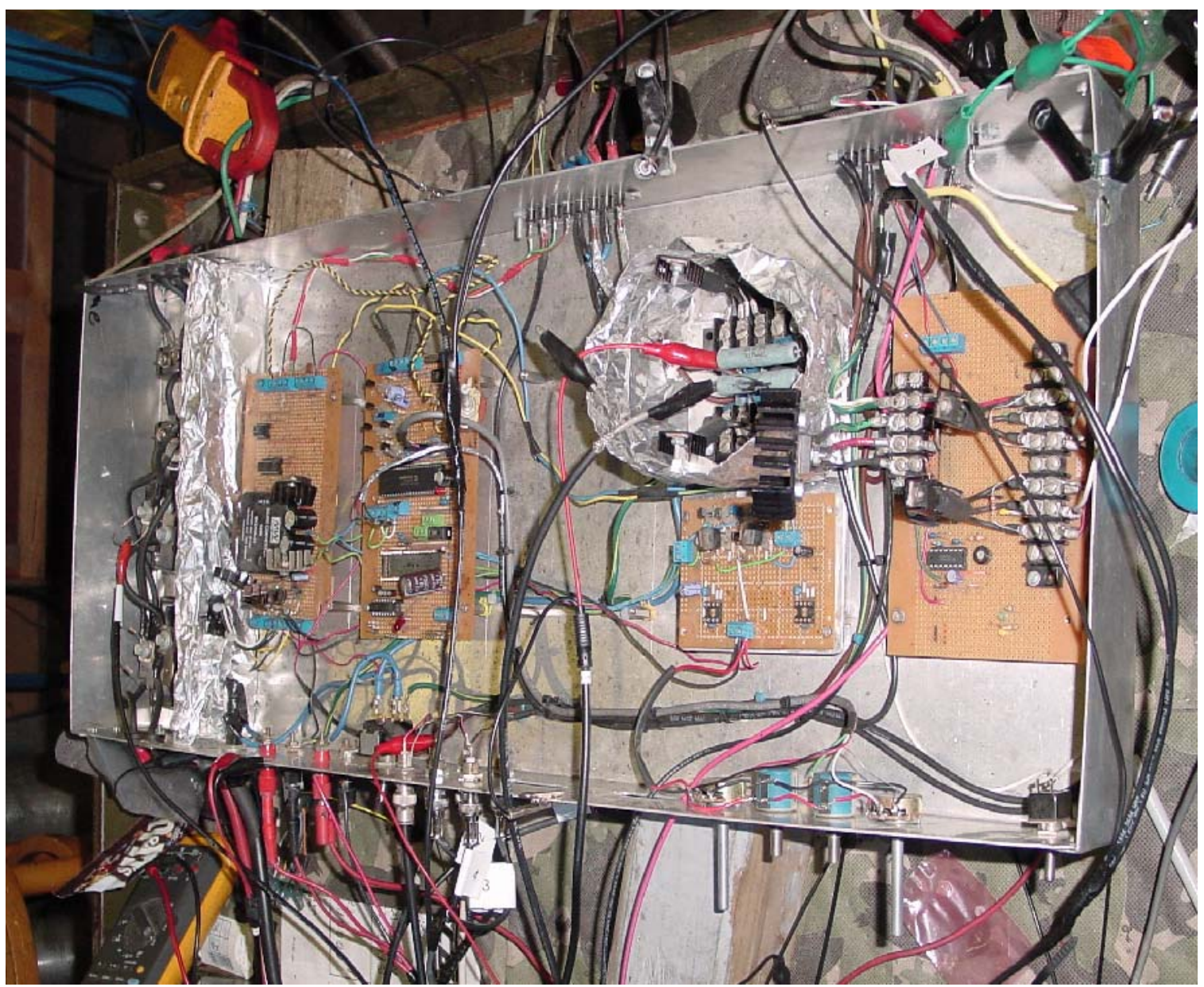

Figure 47. The control box of the linear engine. The IGBTs are shielded in the left hand side of the box. The panels from left to right are: IGBT drivers, micro-controller, injector drivers, and ignition drivers. The potentiometers in the bottom right corner are mean, injection position, spark position, and fueling rate. 


\subsection{Starting the engine}

Until the linear alternator was designed and manufactured the moving part of the alternator was replaced with an iron slug and the stator part was replaced with two coils. This solenoid system provided the starting motion of the translator. The iron slug, with the connecting rod and the pistons, was moved by the two coils being turned on alternately at about $20 \mathrm{~Hz}$. The starter solenoids were supplied from automotive batteries via isolated gate bipolar transistors (IGBTs) (part \#: CM 200DY-24H). The controlling signal to the IGBTs was provided from a signal generator. This method allowed the engine to have a variable cranking frequency to find the system's natural frequency that resulted in the highest compression ratio. Using $60 \mathrm{~V}$ at $20 \mathrm{~Hz}$ cranking frequency, the compression ratio achieved was less than 4:1. This did not prove sufficient to start the engine on compression ignition. In diesel engines 15:1 is typically the required compression ratio to ensure operation. A sparking starting aid was necessary because the "starter motor" was unable to deliver a high enough compression ratio to start the engine. The spark helped combust the fuel injected while starting the engine, igniting the charge at lower compression ratios. It was expected that as part of the starting process, the spark timing would have to be retarded gradually. As a result the engine would continuously build up compression ratio until the charge would autoignite before the spark occurred then spark could be turned off. In reality, there is need for only one spark to start a properly tuned linear engine because the power of the combustion on one side provides energy for proper compression ratio in the other cylinder for the fuel to autoignite and for the engine to sustain operation. The same logic would suggest that only one solenoid would be sufficient to start the engine providing a single dynamic force on the slug. 
However, the solenoids applied at this moment are not strong enough to provide the single stroke necessary to start the engine. Instead, the two solenoids built up energy and amplitude in the system over a period of four to six cycles to provide high enough compression for the spark ignition aid. The highest compression ratio was reached after the fourth cranking cycle then the compression ratio decreased to a steady level. The phenomenon can be explained by the overshooting effect that happened when the system's state was changed from still to a steady frequency close to its natural frequency. It can be concluded that when starting the linear engine, cranking should be done in short periods. Long cranking time is disadvantageous while starting the engine for another reason. Cranking was done at $20 \mathrm{~Hz}$, while the engine operated at around $50 \mathrm{~Hz}$. Once the engine fired there was a sudden operating frequency change in engine operation while cranking stayed at the original $20 \mathrm{~Hz}$. This caused the cranking motor to work against the engine periodically instead of helping it to start. Figure 48 shows the position and cranking signal during an unsuccessful attempt starting the linear engine. 


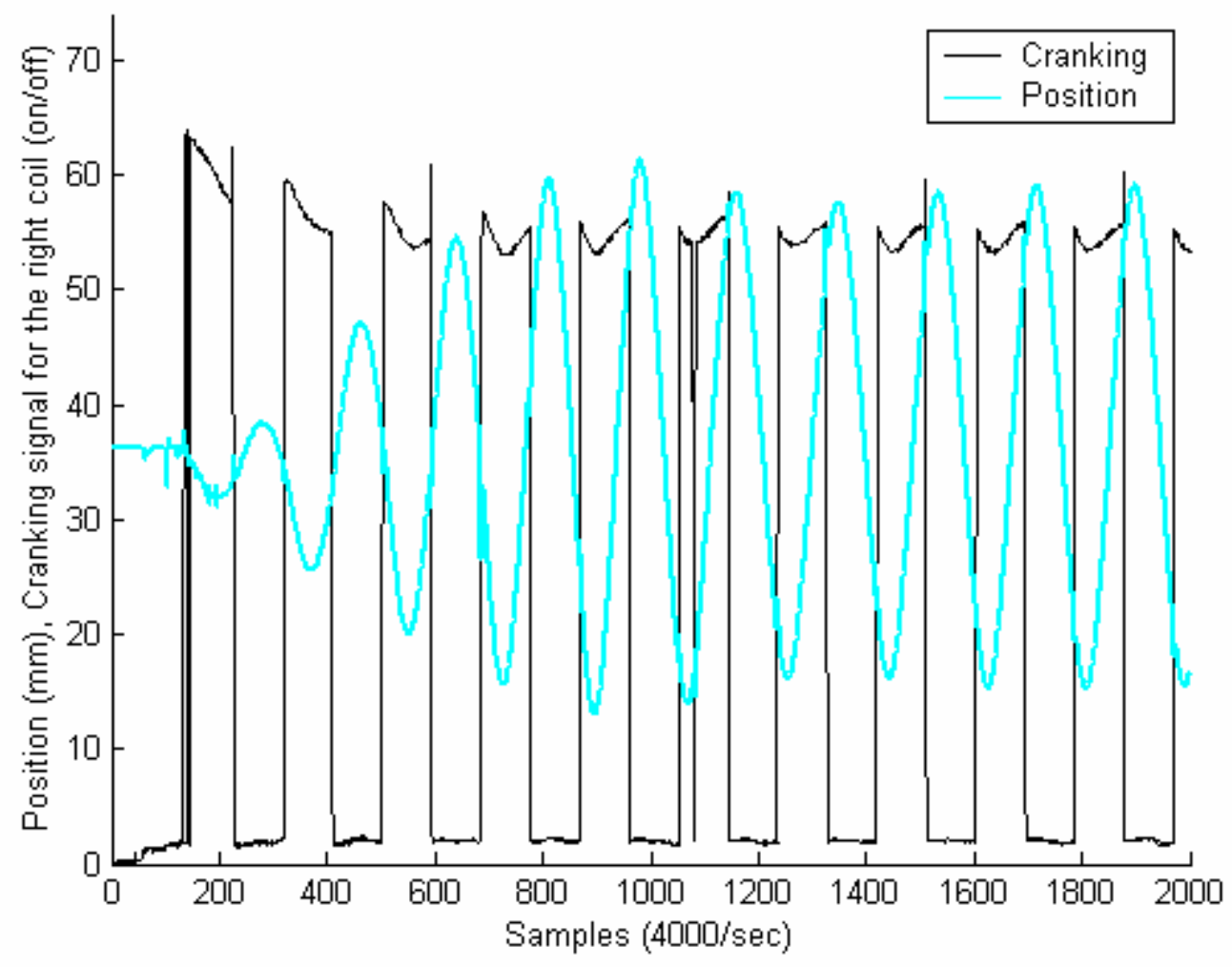

Figure 48. Position and cranking signal during cranking of the engine. Engine did not start.

Figure 49 shows the position and the cranking signal for a successful attempt to start the engine. It can be seen from Figure 49 that the engine did not require the maximum compression ratio that the cranking motor was able to deliver. With the help of the sparking aid, it was not unusual for the engine to start after one or two cycles of cranking. It can also be seen from Figure 49, that the last cycle of cranking was actually stopping the engine. The cranking and the engine operation had different operating frequencies and on that particular cycle they were out of synchronization. 


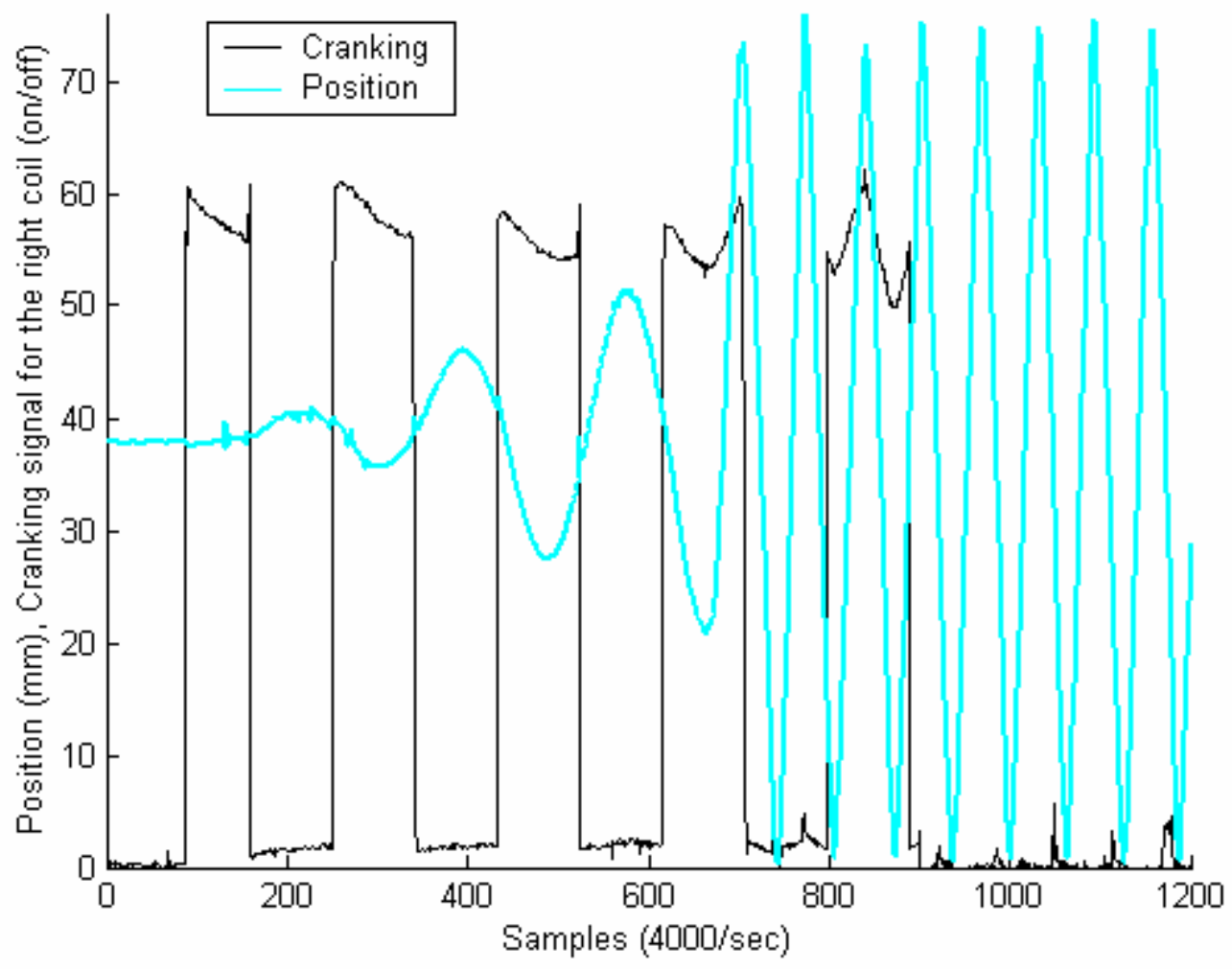

Figure 49. Position and cranking signal during cranking the engine. Engine sustained operation. 


\subsection{Scavenging}

The engine used loop scavenging performed by pressurized (83 kPa) house air. The air was ducted into the engine housing under the piston and the edge of the piston controlled scavenging just like it would in a two-stroke gasoline engine. A person was operating the valve that controlled the amount of air delivered to the engine. This was needed while starting the engine because over-scavenging the engine prevented combustion during starting, but the engine, once running, was running erratically with the amount of air that starting required. This was due to the fact that the highest compression ratio was achieved with cranking at $20 \mathrm{~Hz}$ but the engine operated between 50 and $60 \mathrm{~Hz}$ when it was running requiring three times as much air while running compared to starting. Once the engine was running, full airflow was provided to ensure proper scavenging.

\subsection{Fuel}

Two types of fuel were investigated in the linear engine: diesel fuel and kerosene. The present engine setup was able to operate on kerosene. Experiments were carried out with kerosene only. Although there is no side force on the pistons, two-stroke lubricant was mixed with the fuel in the ratio of 1:50.

Attempts were made to apply diesel fuel as well but the need for a sparking starting aid and the lower volatility of diesel fuel did not allow the engine to run with the present setup. Diesel fuel fouled the sparkplugs before the engine could start and sustain operation. 


\subsection{Load}

As the alternator was still under construction a friction brake was applied to load the engine. The friction brake acted on the rod that connected the two pistons. Figure 50 shows the load implementation in practice.

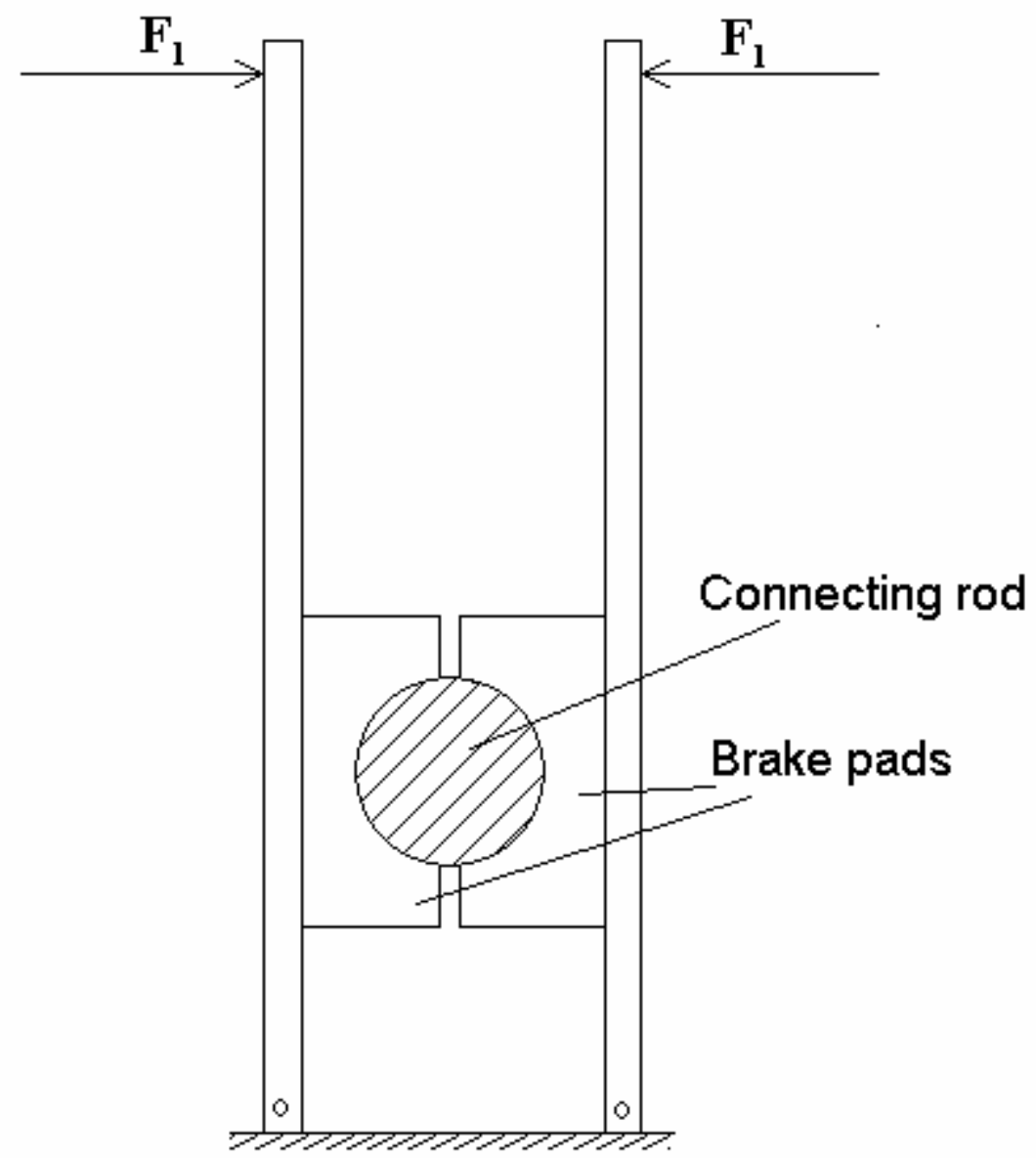

Figure 50. The testing dynamometer for the linear engine was a friction brake on the connection rod.

The two arms of the brake were cable-operated and tension force $\left(\mathrm{F}_{l}\right)$ in the cable was measured with an Interface load cell (part \#: SMT2-450) and recorded by the data acquisition system (DAQ). The friction brake was calibrated with different weights 
applied on the piston-load assembly through a rope and pulley mechanism. Weight and the output voltage of the load cell were recorded.

\subsection{Data acquisition system (DAQ)}

The data acquisition was accomplished by using a National Instrument DAQ6020E data acquisition card and high-speed data acquisition program applied on a personal computer. The high-speed data acquisition program was written by Pertl [75] WVU. The maximum speed of DAQ-6020E was 500 kilo-samples per second. Data were taken at 4000 samples per second during the initial experiments then later at 10,000 samples per second for increased resolution. The data taken included translator position, cylinder pressure, injector current, sparking signal, cranking current, load, and exhaust pressure.

\subsection{Position measurement}

A non-contact inductive-potentiometric displacement sensor was used to detect the position of the translator (MICRO-EPSILON, part \# VIP-100-ZA-2-SR-1). The sensor rod contained a segmented coil. Current flowing through the coil segments were individually measured. A non-contacting aluminum ring attached to the translator moved on the rod. The current flowing in the coil caused eddy currents in the aluminum ring. The eddy currents in turn influenced the magnetic field and the current flowing in the coil segments. The sensor had a $100 \mathrm{~mm}$ measuring range and $0.2 \%$ linearity.

Figure 51 shows the placement of the position sensor. 


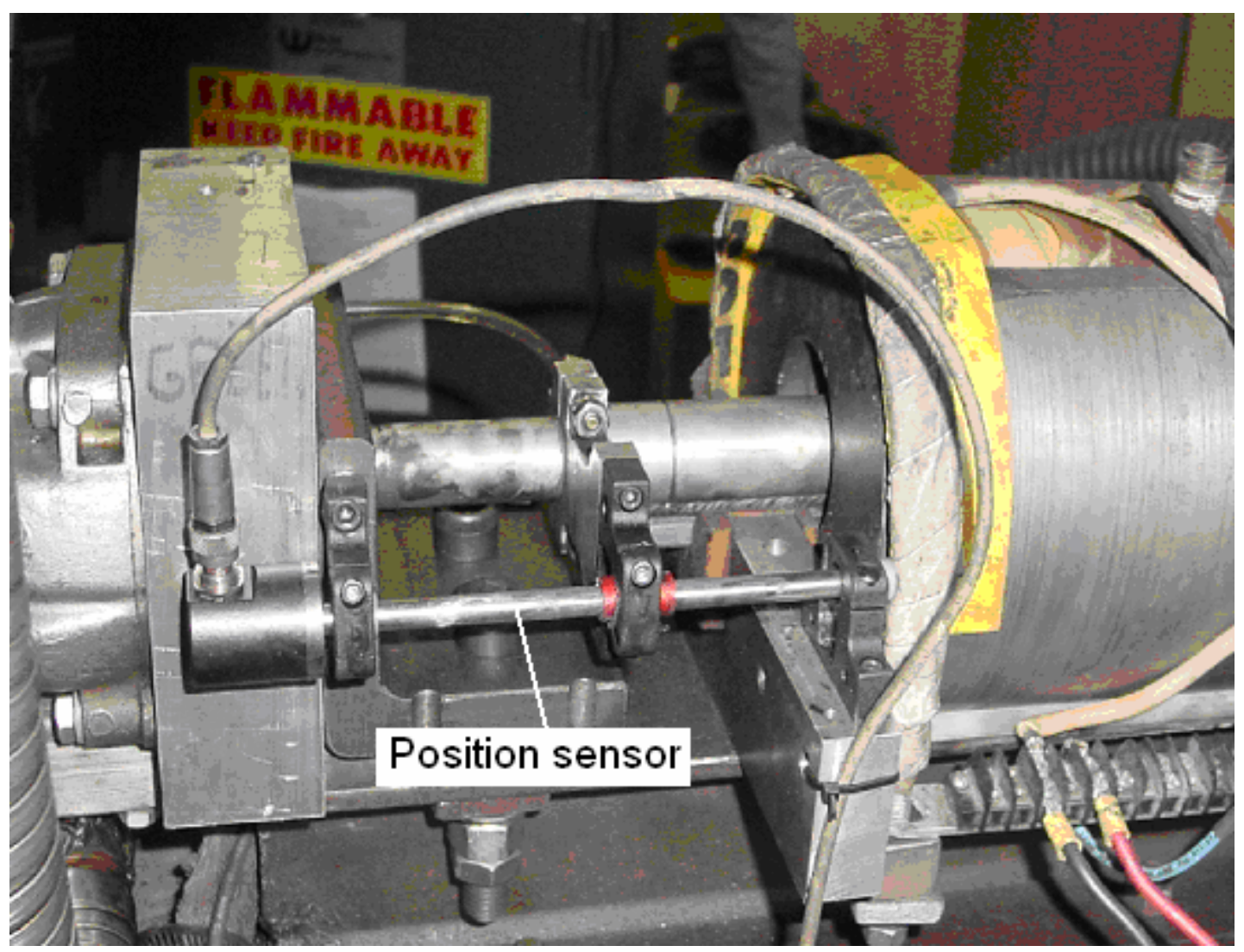

Figure 51. The position sensor. (part \# VIP-100-ZA-2-SR-1)

There was an error introduced in the position measuring method. This error was believed to be due to three factors.

Firstly, because of space limitations, the aluminum ring was mounted on a relatively long and thin aluminum web. The web deflected at sudden speed changes. This effect was the most amplified at the two ends, which caused a longer detected stroke for the engine then its actual stroke was.

Secondly, the connecting rod was a relatively long and thin rod that had changed its length under compression. The strain effect calculations can be seen in Equation 21 Equation 23. The force (F) acting on the half of the rod length is calculated from the 
mass of one piston, half of the rod and the iron slug $(\mathrm{m}=2 \mathrm{~kg})$ and the acceleration of the translator $\left(\mathrm{a}=100,000 \mathrm{~m} / \mathrm{s}^{2}\right)$. The acceleration value was obtained from simulation data. The strain $(\varepsilon)$ was calculated from the force $(F)$, cross-sectional area of the rod $(A=506$ $\mathrm{mm}^{2}$ ), and the modulus of elasticity ( $\left.E=70 \mathrm{GPa}\right)$. The change of rod length $(\Delta \mathrm{x})$, which is the possible error in position measurement, was calculated from the strain $(\varepsilon)$ and the third of the rod length $(x=0.25 \mathrm{~m})$. Figure 52 shows the free body diagram of the translator. The weight of the rod and the pistons were neglected for this calculation.

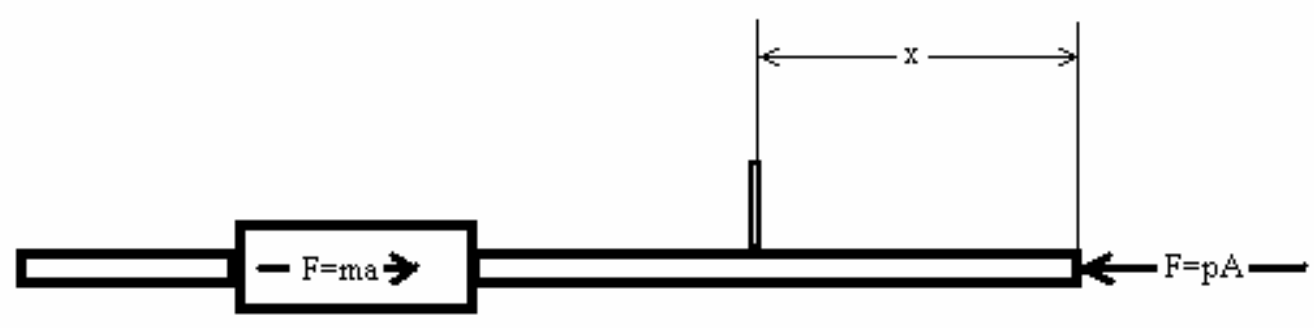

Figure 52. Free body diagram of the translator.

Equation 21

$$
F=m a=2 * 100,000=200,000 N
$$

\section{Equation 22}

$$
\varepsilon=\frac{F}{E A}=\frac{200,000}{\frac{0.0254^{2}}{4} * \Pi * 70 * 10^{9}}=0.00563 m
$$

\section{Equation 23}

$$
\Delta x=\varepsilon X=0.00563 * 0.25=0.0014 \mathrm{~m}=1.4 \mathrm{~mm}
$$

It can be seen from the calculations that there would be a maximum of $1.4 \mathrm{~mm}$ error introduced into the measurement due to strain. 
And thirdly, the cylinders and their fasteners were also experiencing strain as they provided the reaction force for stopping and returning the translator at the end positions.

These three effects have probably happened in a vibrational form and they added or subtracted from each other variably. This caused an inconsistent and unpredictable error that was probably responsible for the high cycle-to-cycle variation in the gathered position data. The measured maximum position data often were outside of range of stroke of the engine. A representative section of the position data can be seen in Figure 53. It can be concluded that the maximum position data varied between 74 and $78 \mathrm{~mm}$ resulting in a $4 \mathrm{~mm}$ window of variance. The minimum data ranged from 0 to $1.2 \mathrm{~mm}$ resulting in a window of $1.2 \mathrm{~mm}$. This large difference in variance was due to the placement of the iron slug and the placement of the moving element of the position sensor. The moving element of the position sensor was placed at about $200 \mathrm{~mm}$ and the slug was placed at about $500 \mathrm{~mm}$ on the $830 \mathrm{~mm}$ long rod. This uneven placement yielded the fact that the error of the position measuring method affected the measurement of the minimum and the maximum value to a different extent. 


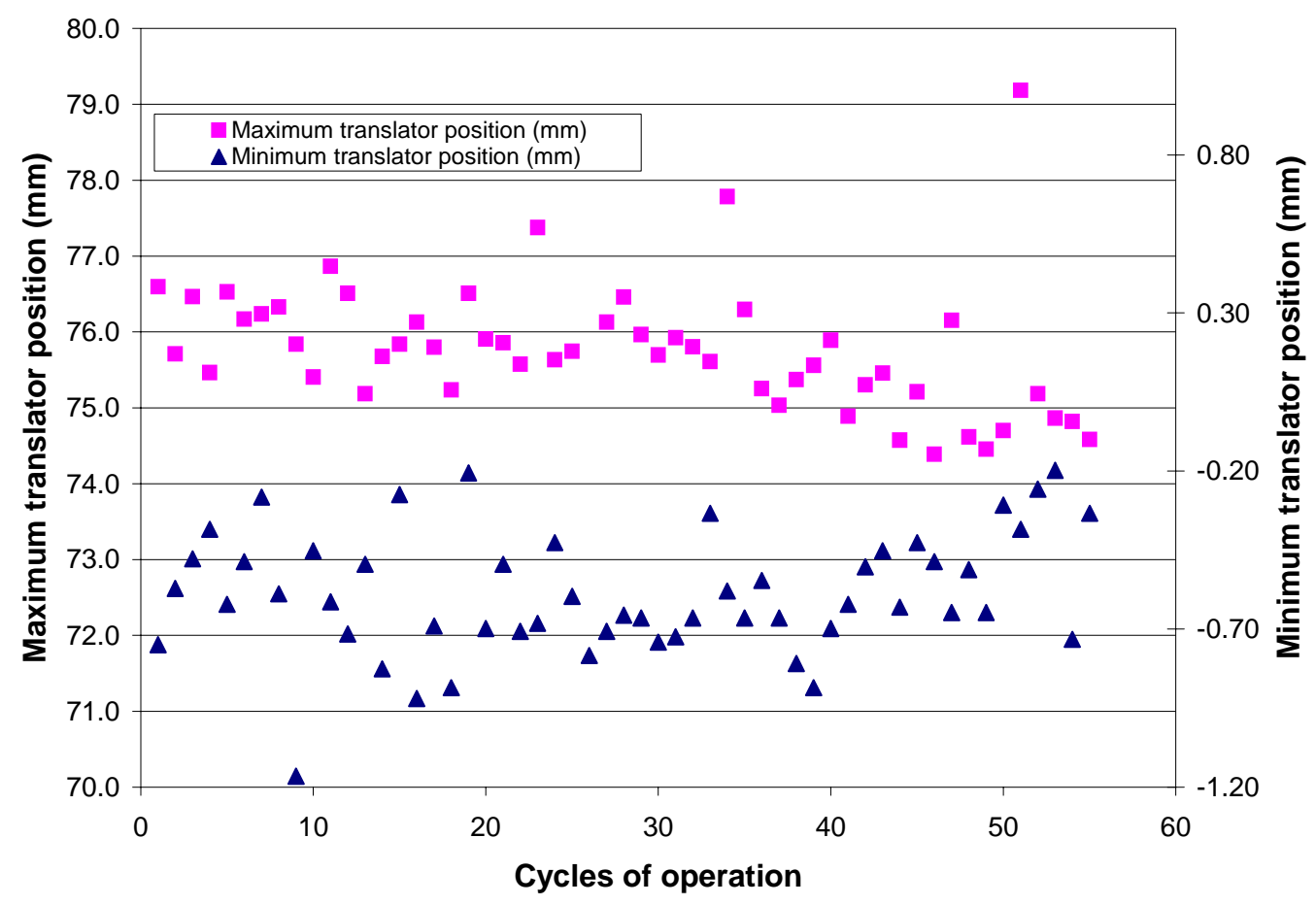

Figure 53. The maximum and minimum value of translator position.

\subsection{Measuring cylinder pressure}

The cylinder pressure was measured with piezo-electric pressure sensors (part \#: PCB JP 145 A01 SN 212). Each cylinder head was equipped with a piezo-electric sensor. The sensor detected pressure in the output form of electric charge. Then a charge-to-voltage converter converted the signal into a readable format. Then the signal was sent through a voltage amplifier and recorded by the data acquisition system.

An error was introduced into the pressure measurement as the temperature change had an effect on the pressure transducers. As temperature changed the zero calibration of the transducers drifted. A computer program was created to compensate for the temperature drift of the pressure transducers. The computer code applied a smoothing function to the pressure data then calculated the rate of pressure change. If the rate of 
pressure change was less than $1 \mathrm{GPa} / \mathrm{sec}$ then the computer decided that that point should be at zero, and moved all the data points from that point forward, inclusive, by the value of the given data point. The value was defined by trial and error. It is clear that this method introduced a certain amount of error in the data sets that probably alters the results of this study but the compensation of the data was unavoidable. The drifting effect of the temperature on the pressure transducers was always present (up to $200 \%$ of the maximum peak pressure in some cases) but of course it had less relative effect on pressure data when the rate of pressure change was large. Figure 54 shows a section of the unmodified and compensated pressure data for both cylinders.

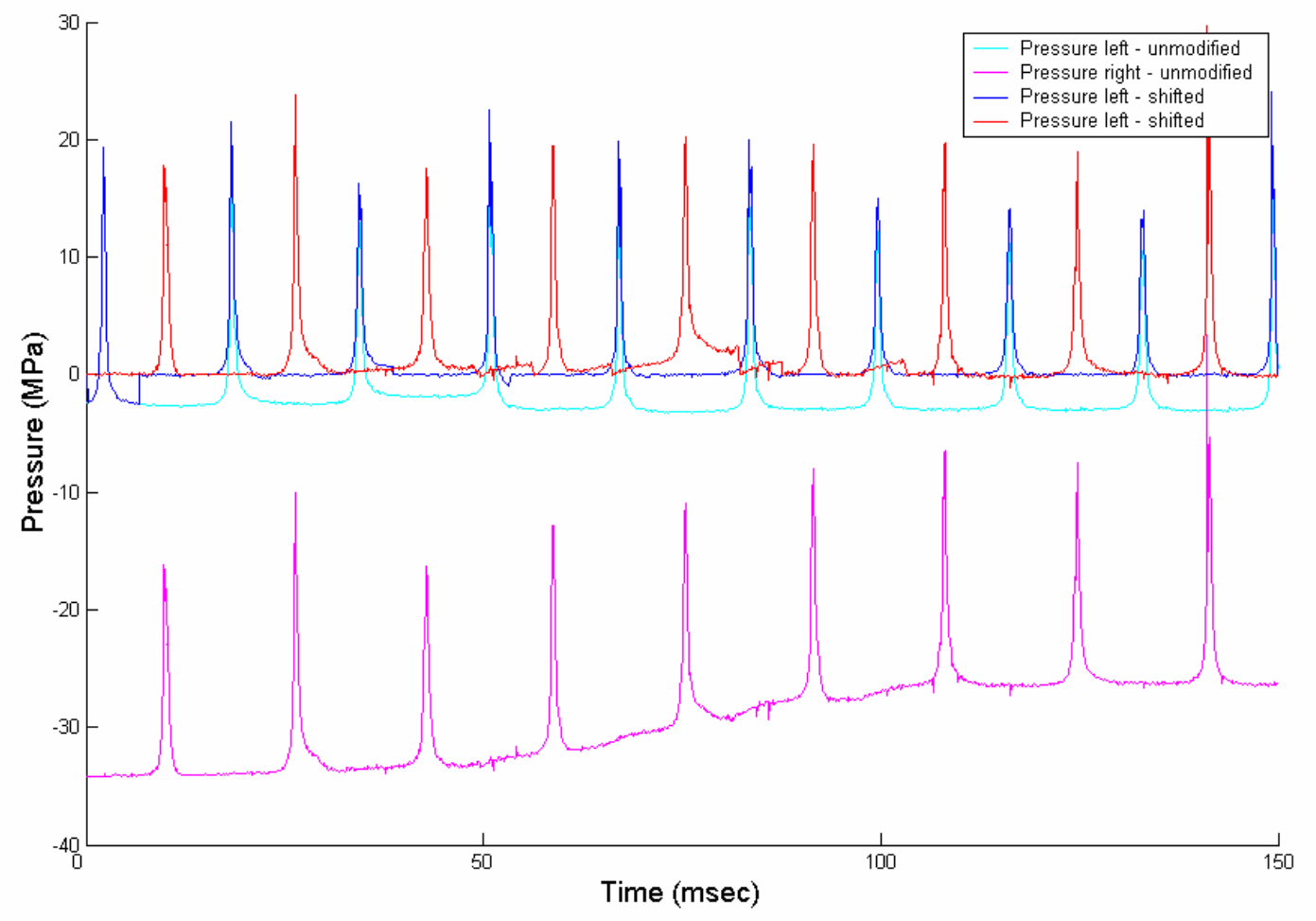

Figure 54. Unmodified and compensated pressure data for both cylinders. Run \# 14. 


\subsection{Conclusions}

The prototype linear engine design was presented with a detailed description of the present state of development. Architecture, control, scavenging process, fuel used in the engine, measuring equipment, and data acquisition system were described. The engine also required a sparking starting aid because the starting solenoids were not powerful enough to provide higher than 4:1 compression ratio. After the engine started, the spark was turned off, and the engine continued to operate on compression ignition principles. It can also be concluded that starting the engine must happen in short bursts rather that long period of cranking taking advantage of the overshooting effect to achieve maximum achievable compression ratio. 


\section{$7 \quad$ Experimental results}

The linear engine was tested to evaluate the effect of translator weight, amount of fuel injected, fueling timing, stroke length, and intake air pressure on engine behavior. Although the engine showed its ability to run and produce output power, it did not demonstrate high reliability. The initial plan was to examine the engine with fractional factorial experiment design method. However, the engine operated with a very high failure rate that was due to two factors. Firstly, the two-stroke gasoline engine components were under designed for the compression ignition diesel application. Secondly, the vibration of the engine caused an excessive fatigue load on the components. These factors resulted in catastrophic failure of various components. Highpressure forces caused failure in pistons, piston pins, the connecting rod, and head gaskets. The inertial force of the moving mass caused failure in the cylinders and bolts and fasteners. The vibration caused the failure of injectors, bolts, fuel lines, and the position sensor. The engine development was a continuous improving and redesigning process. However, the present version of the engine did not allow for long hours of operation but only short minutes or seconds before a break down. This allowed for only a limited testing and prevented the completion of the test matrix. However, the results of the testing are presented in this chapter. Table 3 shows the summary of results for all the "successful" runs that were performed on the linear engine. The table shows results of runs with varied translator mass, intake pressure, injection timing, and stroke length. Detailed analysis of the data follows in this chapter. 
Table 3. Summary of the test runs on the linear engine.

\begin{tabular}{|c|c|c|c|c|c|c|c|c|c|c|c|}
\hline Run number & Stroke length & Exhaust pressure & Moving mass & Fuel injected & Injection position L & Injection position $\mathrm{R}$ & Injection position & Operating frequency & Stalling frequency & Power & Efficiency \\
\hline & $(\mathrm{mm})$ & $(\mathrm{kPa})$ & $(\mathrm{kg})$ & (ml/injection) & $(\mathrm{mm}$ before TDC) & (mm before TDC) & average (mm before TDC) & $(\mathrm{Hz})$ & $(\mathrm{Hz})$ & $(\mathrm{W})$ & $(\%)$ \\
\hline 1 & \multirow{18}{*}{73.8} & \multirow{14}{*}{100 (Ambient) } & \multirow{13}{*}{2.8} & \multirow{7}{*}{0.017} & 20 & 19.5 & 19.75 & 53 & 51 & 520 & 1.25 \\
\hline 2 & & & & & 11 & 16 & 13.5 & 53 & 49 & 700 & 1.75 \\
\hline 4 & & & & & 8 & 15 & 11.5 & 54 & 51 & 1000 & 2.40 \\
\hline 6 & & & & & 6 & 14.5 & 10.25 & 50 & 47 & 700 & 1.83 \\
\hline 7 & & & & & 10 & 11.5 & 10.75 & 49 & 47 & 1200 & 3.13 \\
\hline 3 & & & & & 8 & 10 & 9 & 49 & 48 & 325 & 0.83 \\
\hline 5 & & & & & 0 & 7 & 3.5 & 55 & 53 & 2000 & 4.62 \\
\hline 9 & & & & \multirow{6}{*}{0.0037} & 6 & 23 & 14.5 & 52 & 47 & 740 & 8.87 \\
\hline 10 & & & & & 7 & 22 & 14.5 & 50 & 50 & 400 & 4.50 \\
\hline 11 & & & & & 5 & 16 & 10.5 & 51 & 51 & 60 & 0.66 \\
\hline 13 & & & & & 9 & 8 & 8.5 & 50 & 50 & 0 & 0.00 \\
\hline 12 & & & & & 2 & 12 & 7 & 54 & 54 & 0 & 0.00 \\
\hline 8 & & & & & 5 & 22 & 13.5 & 60 & 49 & 2800 & 32.18 \\
\hline 21 & & & 4.7 & 0.017 & 20 & 18 & 19 & 35 & $\mathrm{n} / \mathrm{a}$ & $\mathrm{n} / \mathrm{a}$ & $\mathrm{n} / \mathrm{a}$ \\
\hline 14 & & \multirow{4}{*}{127.2} & \multirow{4}{*}{2.8} & \multirow{4}{*}{0.0037} & 8 & 20 & 14 & 61 & 51 & 1750 & 19.32 \\
\hline 15 & & & & & 8 & 22 & 15 & 63 & 47 & 1550 & 18.57 \\
\hline 16 & & & & & 4 & 16 & 10 & 63 & 53 & 1080 & 11.47 \\
\hline 17 & & & & & 0 & 14 & 7 & 59 & 55 & 0 & 0.00 \\
\hline 19 & \multirow{3}{*}{78.3} & \multirow{3}{*}{100} & \multirow{3}{*}{2.8} & \multirow{2}{*}{0.0122} & 13 & 13 & 13 & 47 & $\mathrm{n} / \mathrm{a}$ & $\mathrm{n} / \mathrm{a}$ & $\mathrm{n} / \mathrm{a}$ \\
\hline 20 & & & & & 7 & 9 & 8 & 48 & $\mathrm{n} / \mathrm{a}$ & $\mathrm{n} / \mathrm{a}$ & $\mathrm{n} / \mathrm{a}$ \\
\hline 18 & & & & 0.0037 & \multicolumn{2}{|c|}{ Gradually retarded till stall } & -1 & 45 & $\mathrm{n} / \mathrm{a}$ & $\mathrm{n} / \mathrm{a}$ & $\mathrm{n} / \mathrm{a}$ \\
\hline
\end{tabular}




\subsection{Definition of terms}

-Load was a friction brake applied on the connecting rod. It was gradually increased until the engine stalled. The gradual application of load was sufficiently slow thus it can be assumed that load was steady when the engine stalled.

-Operating frequency was the frequency at which the engine operated with no load applied on the engine.

-Stalling frequency was the frequency of the engine operated immediately before it stalled.

-Effective stroke length was the distance between the upper edge of the exhaust port and the cylinder head.

-Total stroke length was the distance that the translator could actually travel cylinder head to cylinder head.

- Injection timing was defined by the piston's position in the cylinder. Injection timing before TDC meant the distance the piston would have to travel before it would impact the cylinder head at the moment the injector current increased from $0 \mathrm{~V}$ to $20 \mathrm{~V}$.

-Top dead center (TDC) was the position of the piston where it impacted the cylinder head. In theory and under normal working conditions, the piston should never reach TDC in the sense it was used here.

-Mechanical efficiency was calculated from the power output of the engine divided by the energy in the fuel injected over a period of time.

-Power output of the engine was calculated from the friction force acting on the translator divided by the average speed of the translator.

\subsection{Effect of the moving mass}

Although experiments were planned to run the engine at different weights, only two translator weights were investigated $2.8 \mathrm{~kg}$ and $4.7 \mathrm{~kg}$. The translator weight was varied by changing the material of the connecting rod. Table 4 shows the mass of the translator with the aluminum and the steel shaft and the resulting operating frequency of 
the engine. The aluminum shaft resulted in an operating frequency of approximately 50 $\mathrm{Hz}$ and the steel shaft resulted in $35 \mathrm{~Hz}$.

Table 4. The effect of translator mass, different shaft materials, on the operating frequency. (experimental results)

\begin{tabular}{|c|c|c|}
\hline Shaft material & Mass of the translator $(\mathrm{kg})$ & Operating frequency $(\mathrm{Hz})$ \\
\hline Aluminum & 2.8 & 50 \\
\hline Steel & 4.7 & 35 \\
\hline
\end{tabular}

The translator mass was composed of the mass of the pistons, rings, wristpins, the iron slug, the connecting shaft, and the dead weight attached to the shaft for the experimentation. The weight of the pistons, rings, and pins were constant, leaving the shaft and the dead weight as the possibility for varying weight. Dead weight was attached to the shaft using different bonding methods however, every attempt resulted in failure. Anything that was welded to the shaft broke off in a matter of seconds so welding was quickly out of question. Drilling into the shaft reduced its resistance to buckling if large pins were chosen on one hand, and on the other hand if pins were small they simply did not stand the shear stress. The weight that stayed on the shaft long enough to record some data were too small to have any noticeable effect on engine operation. This left one option to change translator mass, namely the connecting shaft. The diameter of the shaft was preset by the space in the piston to a maximum of $25.4 \mathrm{~mm}$ (1 inch), which was the diameter that fit in the piston. The length of the shaft was kept at a minimum to reduce the chance of buckling. The solution to the problem was to change the material of the shaft. The materials used were aluminum and steel yielding weighing $2.8 \mathrm{~kg}$ and $4.7 \mathrm{~kg}$ respectively. Extensive testing was performed on the engine with the aluminum shaft because the steel shaft has proven to be too heavy for the other components of the engine. The application of the steel shaft resulted in catastrophic 
failure in the cylinders. The cast aluminum of which the cylinders were made was not strong enough to stand the stresses that the steel shaft, moving at $35 \mathrm{~Hz}$, caused for an extended period of time. The Kawasaki cylinders were bolted to base plates on the two sides and the reaction force that stopped the translator at the end positions detached the cylinder on one side from its base plate. At the same time the translator destroyed the piston on the other side impacting into the other base-plate. The engine with the steel shaft operated just long enough to determine the operating frequency before it failed.

\subsection{Effect of the stroke length}

The effective stroke length is the distance that the piston can travel from the upper edge of the exhaust port to the cylinder head. The effective stroke was constant as it was predefined by the cylinder geometry. Total stroke length was the distance that the translator can travel from cylinder head to cylinder head. The total stroke length was varied by varying the length of the connecting shaft, thus varying the distance between the pistons. Different shaft lengths resulted in different free travel distances of the translator. Free-travel is the distance that the translator travels having no pressure on either piston. In other words, it is the distance from the point where the piston starts revealing the exhaust port on one side, resulting in blow-down of the gases, to the piston fully covers the exhaust port on the other side, starting compression. Three different stroke lengths were investigated defined by free-travel of 5,0 , and $-5 \mathrm{~mm}$. As it was expected, the longer stroke resulted in lower operating frequency than the shorter stroke. This was due to two facts. In the case of the longer stroke the translator had to travel a longer distance that took longer time and resulted in decreased operating frequency. Furthermore, the free travel part consumed energy that slowed down the piston's motion. 
During these tests no load was applied. Table 5 shows the effect of stroke length on the operating frequency. The negative value of free-travel indicates that there is an overlapping period where there is pressure over both pistons at the same time. In this particular case the piston on one side started compression and after $5 \mathrm{~mm}$ the piston on the other side revealed the exhaust port and started blow-down. However, the cranking was not sufficient enough to start the engine with $5 \mathrm{~mm}$ overlap.

Table 5. The effect of stroke length on the operating frequency. (experimental results)

\begin{tabular}{|c|c|c|}
\hline Stroke length $(\mathrm{mm})$ & Free-travel $(\mathrm{mm})$ & Operating frequency $(\mathrm{Hz})$ \\
\hline 78 & 5 & 48 \\
\hline 73 & 0 & 50 \\
\hline 68 & -5 & Engine did not start \\
\hline
\end{tabular}

\subsection{Effect of intake air pressure}

Intake air was supplied from an external source using house air (83 kPa). Changing the intake air pressure did not have effect on the engine for a simple reason: the piston closed the intake port before it closed the exhaust port. This caused the pressure in the cylinder to drop to ambient as soon as the intake port was covered. As a conclusion cylinder pressure was independent of intake pressure. However, restriction on the exhaust pipe resulted in increased pressure captured in the cylinder. Two cases were examined. One was with no restriction resulting in ambient pressure in the cylinder at port closure; the other was with restriction on the exhaust pipe resulting in $127 \mathrm{kPa}$ pressure in the cylinder at port closure. The effect of overcharging was examined on three variables: operating frequency, stalling frequency, and output power. For this experiment the fueling rate was set at $3.7 \mathrm{l} \mu \mathrm{l} /$ injection (about $140 \mathrm{~J} /$ injection) and injection timing was preset at values of 14.5, 10, and $7 \mathrm{~mm}$ before TDC. Injection timing was defined by the 
piston's position in the cylinder. Injection timing before TDC meant the distance the piston would have to travel before it would impact the cylinder head at the moment the injector current increased from $0 \mathrm{~V}$ to $20 \mathrm{~V}$. For this study, the load was continuously increased until the engine stalled. The operating frequency indicated the frequency of engine operation with no load and stalling frequency indicated the frequency, at which the engine ran just before it stalled.

Table 6. The effect of overcharging on output power, operating frequency and stalling frequency at different injection timings. (experimental results)

\begin{tabular}{|c|c|c|c|c|c|c|c|c|}
\hline \multirow{2}{*}{$\begin{array}{c}\text { Injection timing } \\
\text { (mm before TDC) }\end{array}$} & \multicolumn{9}{|c|}{ Overcharging pressure (kPa) } \\
\cline { 2 - 11 } & 100 & 127 & 100 & 127 & 100 & 127 & 100 & 127 \\
\cline { 2 - 10 } & \multicolumn{2}{|c|}{ Output power (W) } & Operating frequency (Hz) & Stalling frequency (Hz) & \multicolumn{3}{|c|}{ Efficiency (\%) } \\
\hline 14.5 & 570 & 1650 & 51 & 62 & 48.5 & 49 & 6.68 & 18.94 \\
\hline 10 & 60 & 1080 & 51 & 63 & 51 & 53 & 0.01 & 11.47 \\
\hline 8 & 0 & 0 & 54 & 59 & 54 & 55 & 0.00 & 0.00 \\
\hline
\end{tabular}

Figure 55 through Figure 58 show the effect of overcharging on output power, operating frequency, and stalling frequency respectively. The increasing charging pressure resulted in increased output power, efficiency, operating frequency, and stalling frequency. It is noted that only two data points are shown. The line connecting the two points is to illustrate the relative change of data, not to specify a functional relationship. 


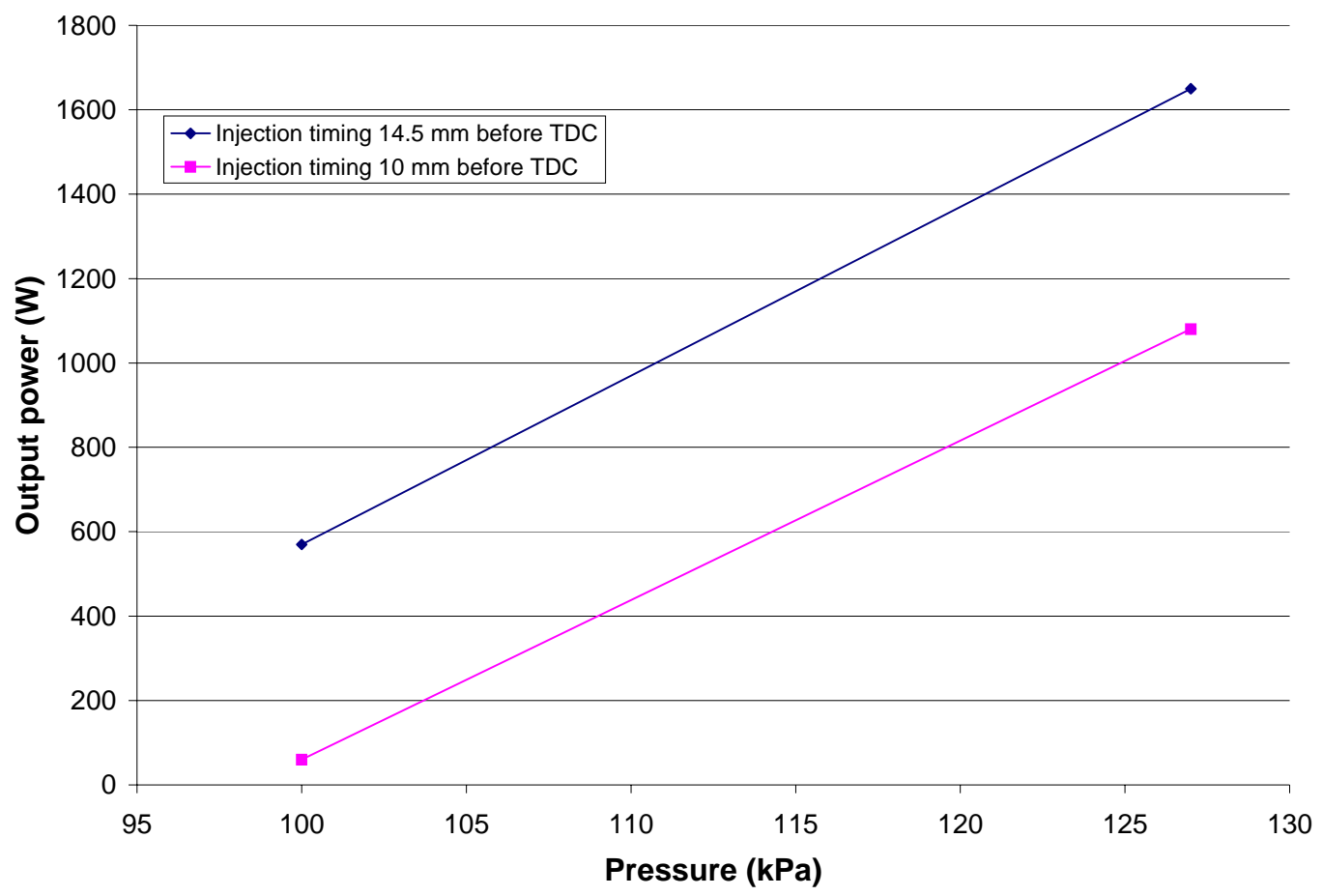

Figure 55. The effect of overcharging on output power for different injection timings. Increased charging pressure results in increased power output. (Experimental results)

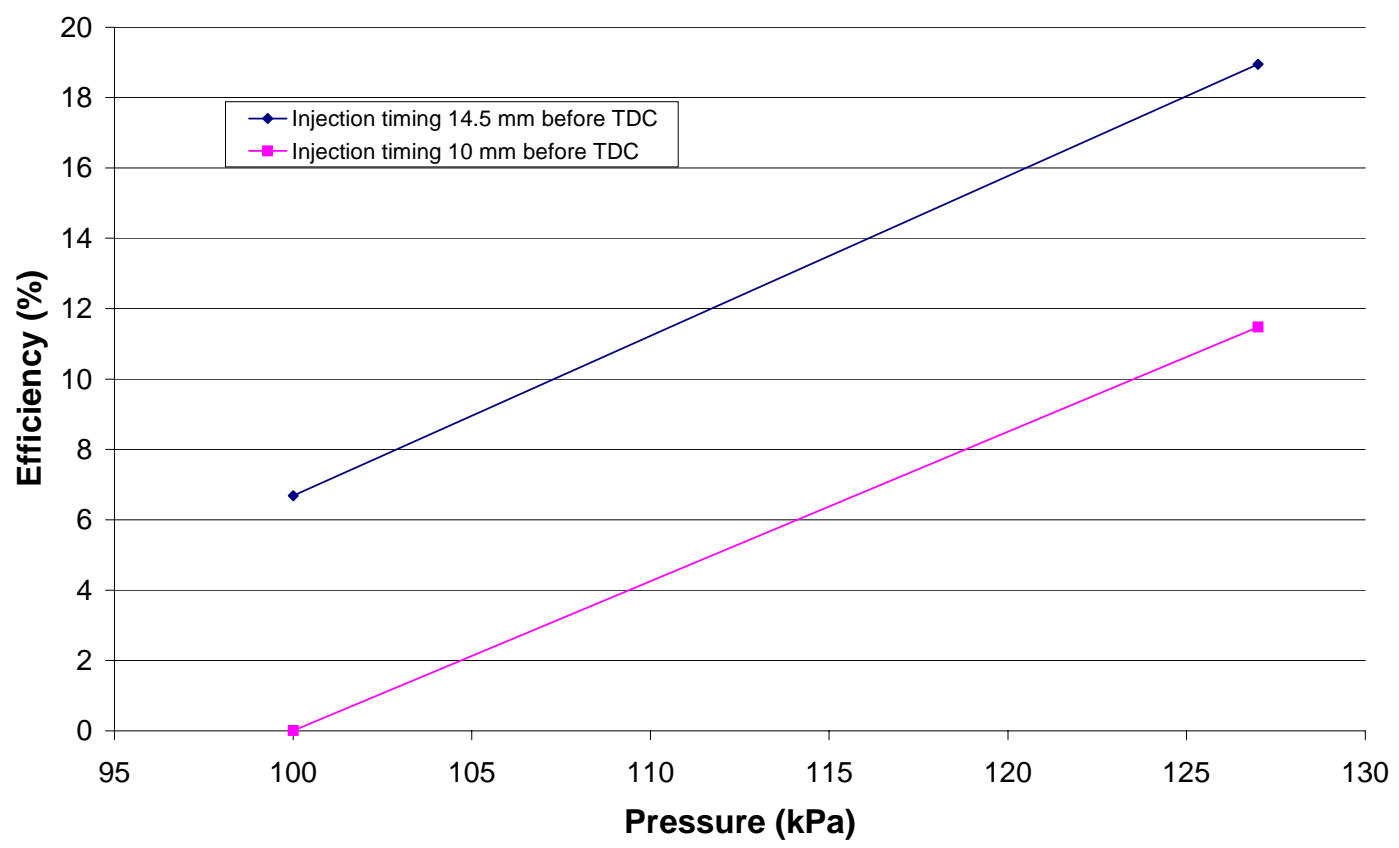

Figure 56. The effect of overcharging on efficiency for different injection timings. Increased charging pressure resulted in increased efficiency. (Experimental results) 


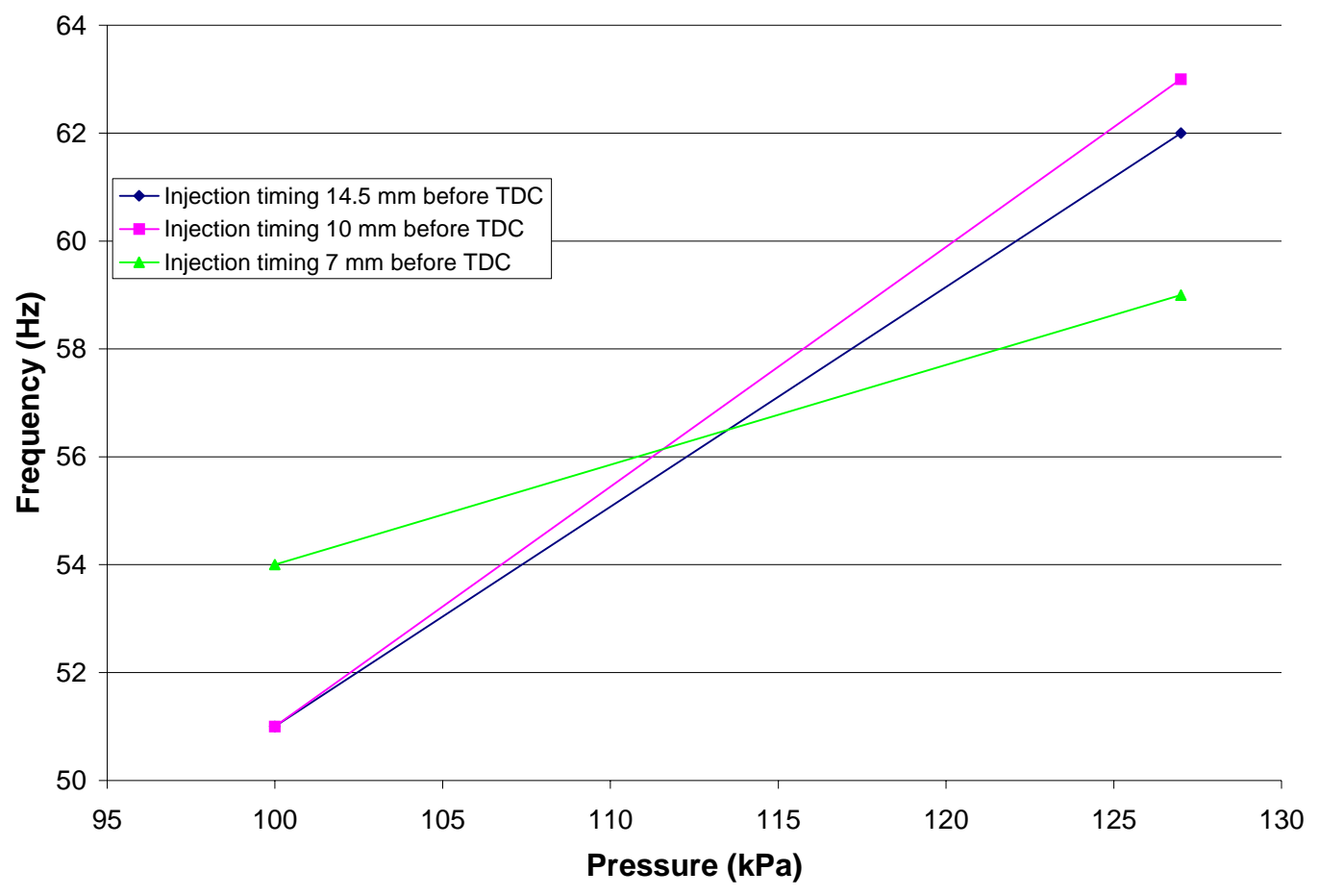

Figure 57. The effect of overcharging on operating frequency for different injection timings. Increased charging pressure resulted in higher operating frequency. (Experimental results)

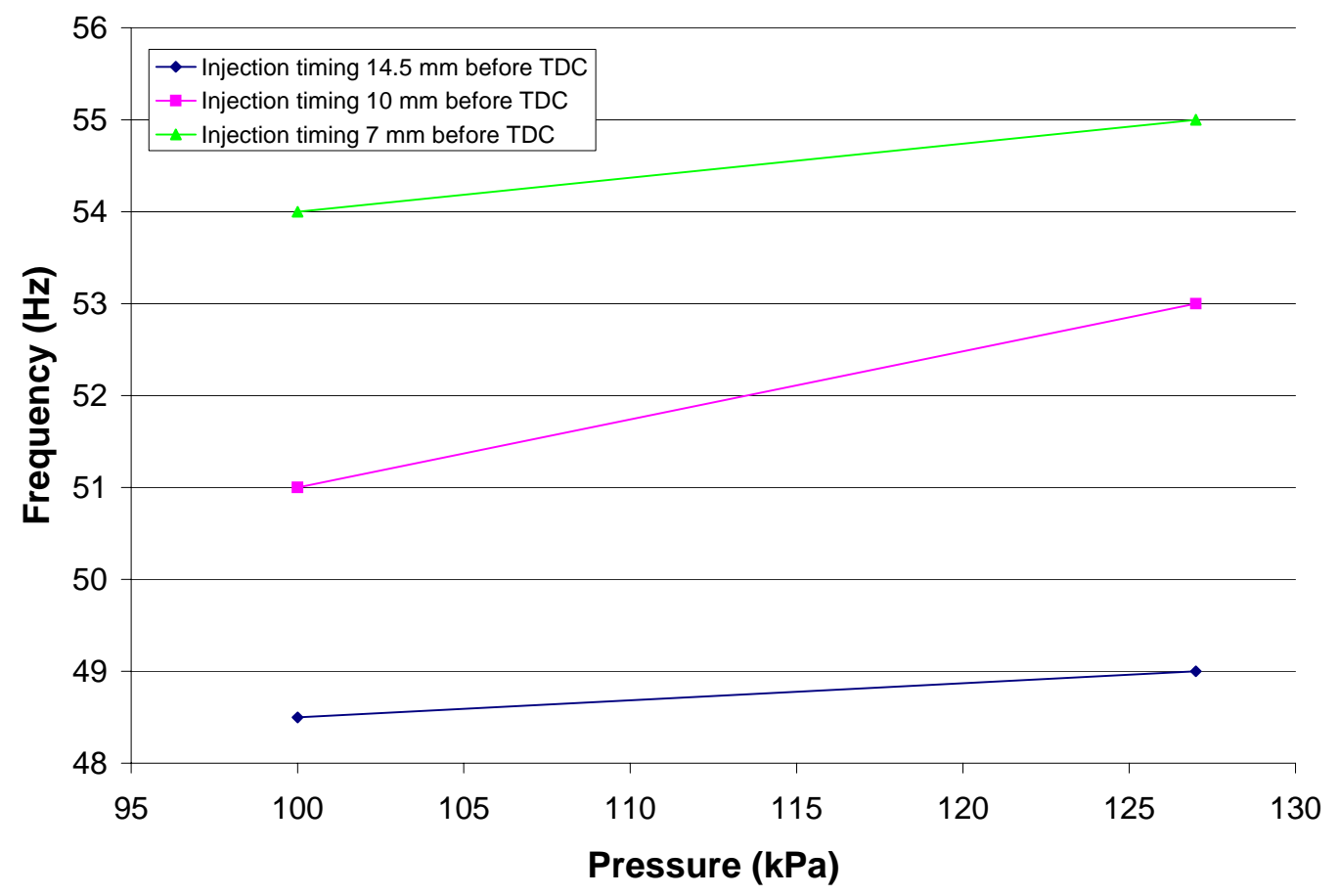

Figure 58. The effect of overcharging on stalling frequency for different injection timings. Increased charging pressure resulted in higher stalling frequency. (Experimental results) 
The increased exhaust backpressure (overcharging the engine) resulted in higher operating and stalling frequency, higher power output, and higher efficiency than those at ambient exhaust pressure. These phenomena were believed to be due to over fueling (the amount of fuel injected was kept constant), which caused the amount of air in the cylinder to control the amount of fuel burned as well as the heat released in the cylinder.

\subsection{Effect of injection timing}

Injection timing is one of the major factors that affects operation in regular slidercrank engines therefore it was expected to have a significant effect on the operation of the linear engine as well. Figure 59 through Figure 61 show the effect of injection timing on the operating frequency, stalling frequency, and output power respectively.

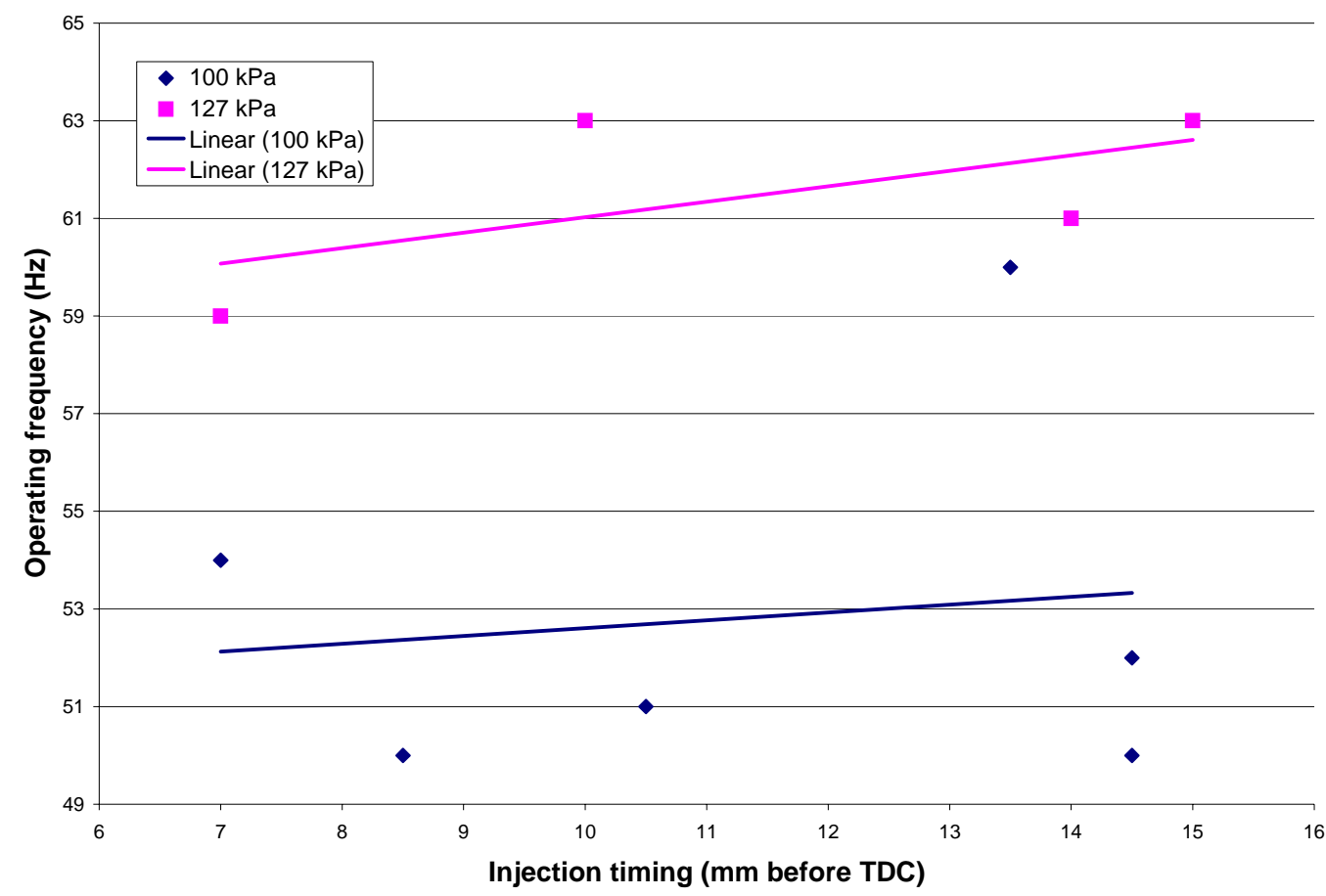

Figure 59. Operating frequency vs. injection timing. Advanced injection timing slightly increased the no load operating frequency. (Experimental results) 


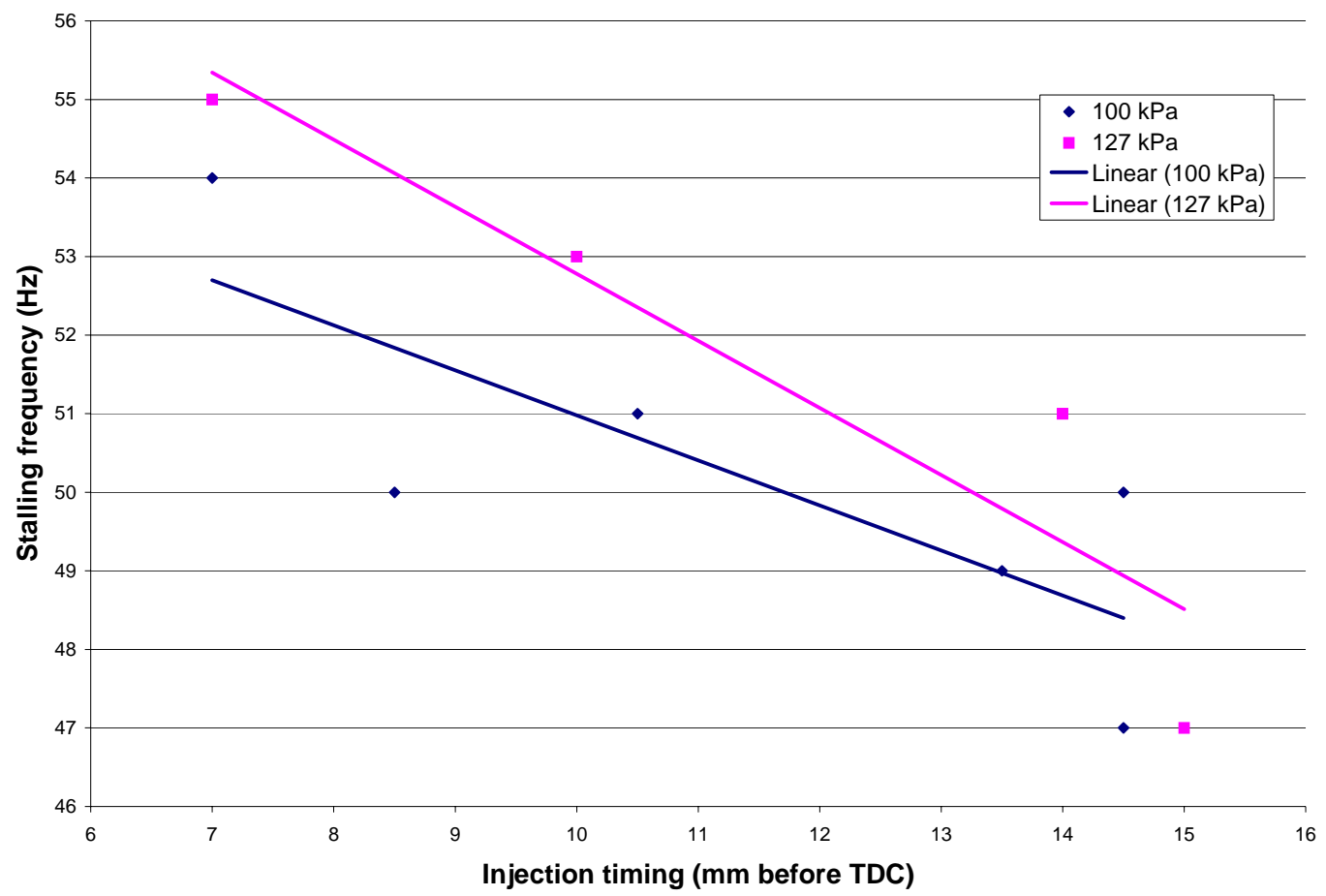

Figure 60. Stalling frequency vs. injection timing. Advanced injection timing resulted in lower stalling frequency. (Experimental results)

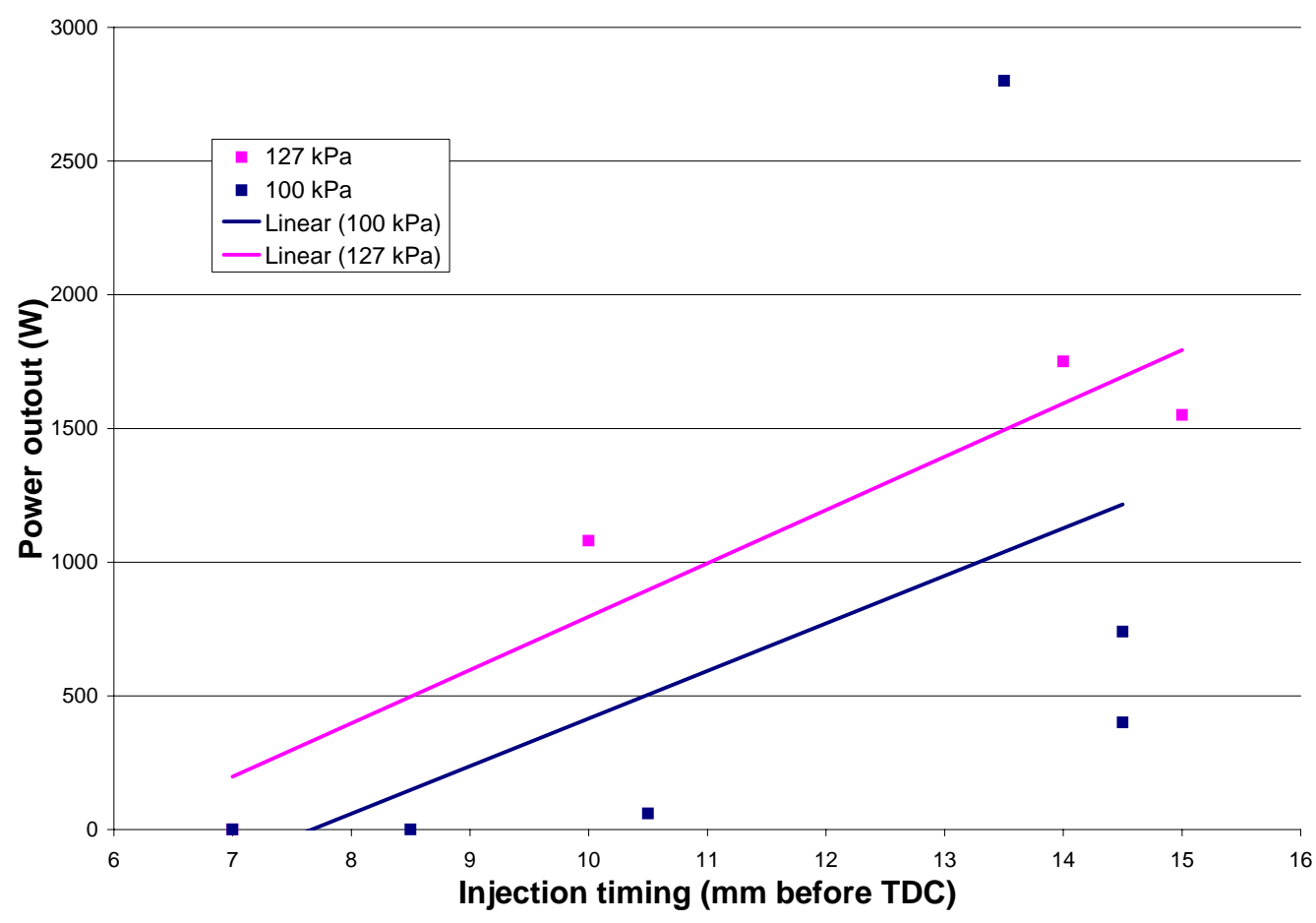

Figure 61. Power output vs. injection timing. Advancing the injection timing resulted in higher power output. (Experimental results) 


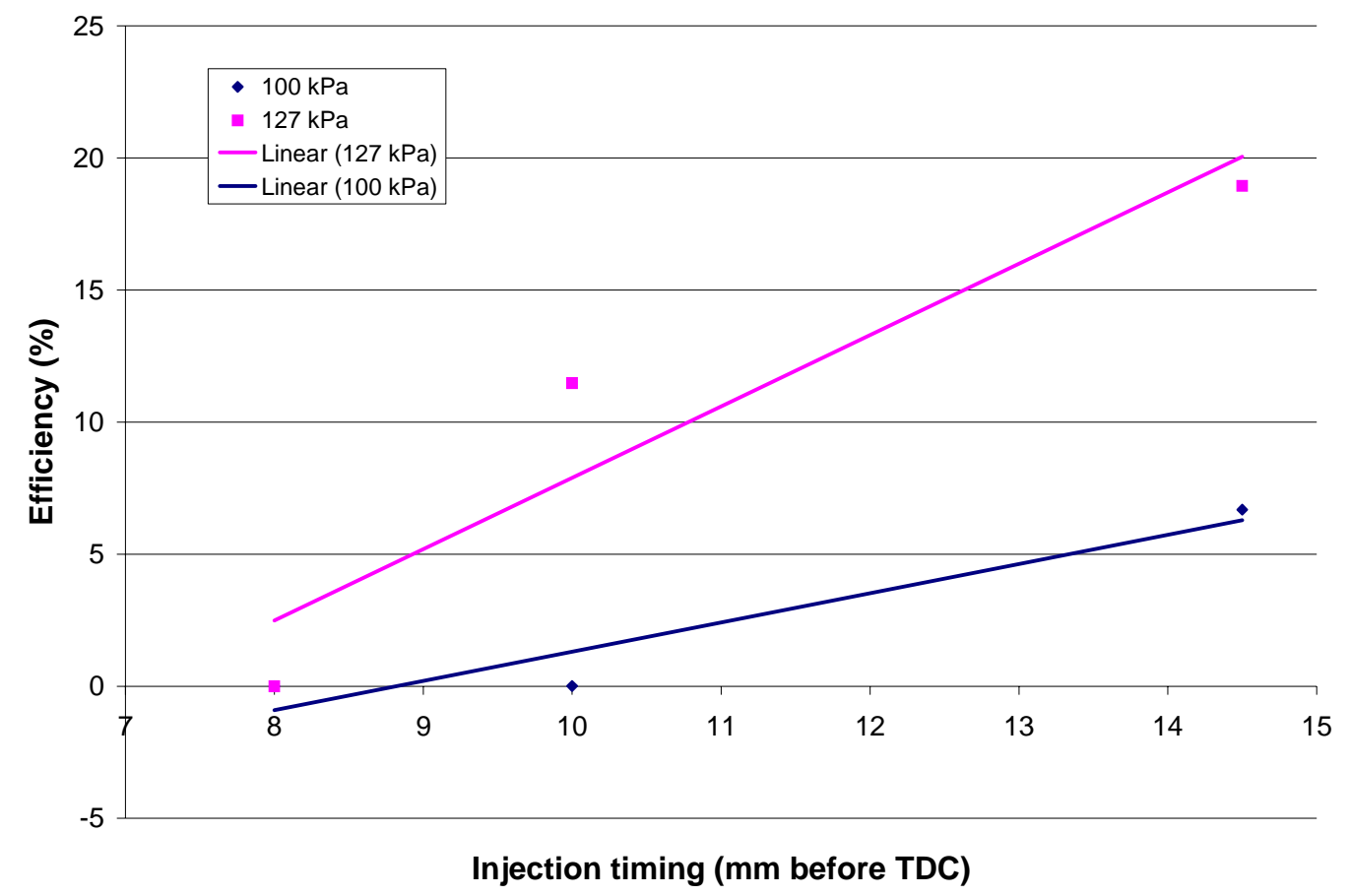

Figure 62. The effect of injection timing on efficiency. Advanced timing resulted in increased efficiency. (Experimental results)

Advancing fuel injection resulted in increased power output, increased efficiency, and decreased stalling frequency. This was due to the fact that early injection resulted in a pseudo-HCCI operation. Operating frequency showed an increasing tendency; however, the high variation in the data did not allow for a definite conclusion.

In case of later injection (close to but still before TDC) the start of ignition was controlled by injection timing just like in a conventional direct injection engine. Combustion rates were believed to be comparable in DICI engines and the linear engine operating in DICI mode. Although the combustion rate can be increased by finer atomization and improved swirl in the cylinder, it still takes time, resulting in a lower efficiency than that of ideal Otto cycle. 
In case of advanced injection, fuel was injected in the cylinder as soon as the exhaust port was closed leaving time for the fuel to evaporate and mix with the air. Ignition was controlled by cylinder conditions. As the piston was moving towards TDC, as soon as pressure and temperature reached critical value, the charge autoignited. The pseudo-HCCI operation caused a larger portion of the fuel to burn instantly yielding a higher efficiency of the engine (closer to ideal Otto cycle efficiency). It was also clear that different operating conditions resulted in different compression ratios, which caused no problem in the operation of the linear engine. For further discussion, see Section 8.4.

\subsection{Effect of load}

As the linear alternator was not available for testing, the load was mimicked by the application of a friction brake on the connecting shaft. The load created by the linear alternator is speed dependent, so was the load created by friction. They are similar except for one major characteristic. The alternator requires no force from the engine at the end positions when the piston stops and returns, as opposed to the friction brake that represents the largest resistance force at the ends when the piston stops for a split second and the friction coefficient changes from dynamic to static. Although the phenomenon of friction coefficient change is present in the case of the friction brake applied to the linear engine, no actual dwelling time could be seen in the data. The undetectably short dwelling time resulted in insignificant increase in blow-by and heat loss therefore it was concluded that the effect of friction coefficient change could be neglected. Virtually no work would be lost because, although the increased friction results in increased resisting force on the translator, gas forces are magnitudes larger than the effect of friction. 
Figure 63 shows the final portion of Run \# 16 in Table 3. After the engine was started and it stabilized operation with a preset fueling rate and injection timing, the friction brake was gradually applied to the connecting shaft until the engine stalled. It is clear from the figure that increasing load caused the frequency to drop. Frequency dropped from $63 \mathrm{~Hz}$, no load condition, to $53 \mathrm{~Hz}$ at maximum load before stall.

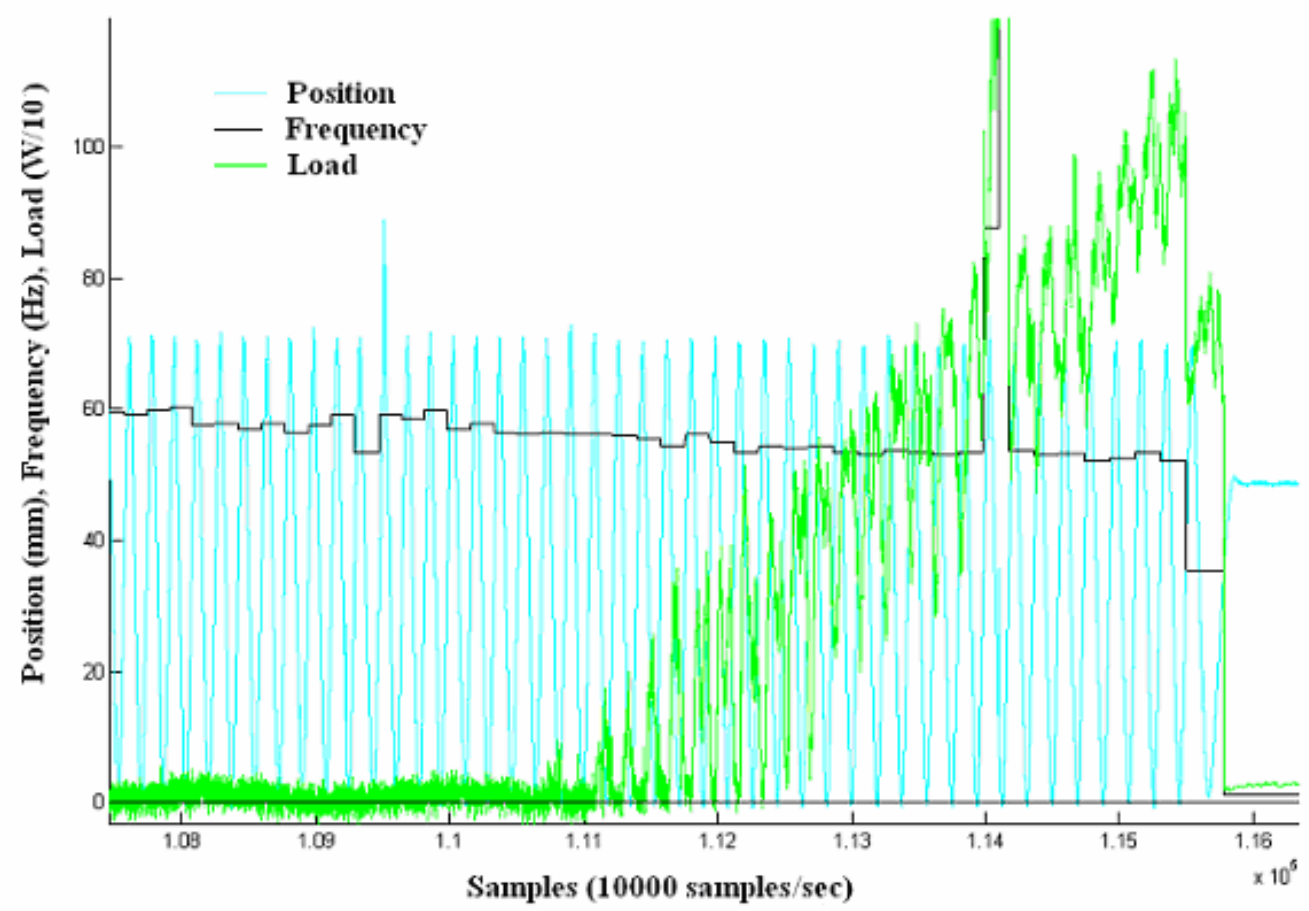

Figure 63. Translator position, operating frequency, and load. Increasing load caused the operating frequency to drop. (Experimental results. Run \# 16 in Table 3)

Figure 64 shows cylinder pressure and load data vs. time. It was expected that peak cylinder pressure would increase with increasing load. However a conclusion could not be drawn from the results, because variation of peak pressure was so high that it made the trend unnoticeable even if there was one. 


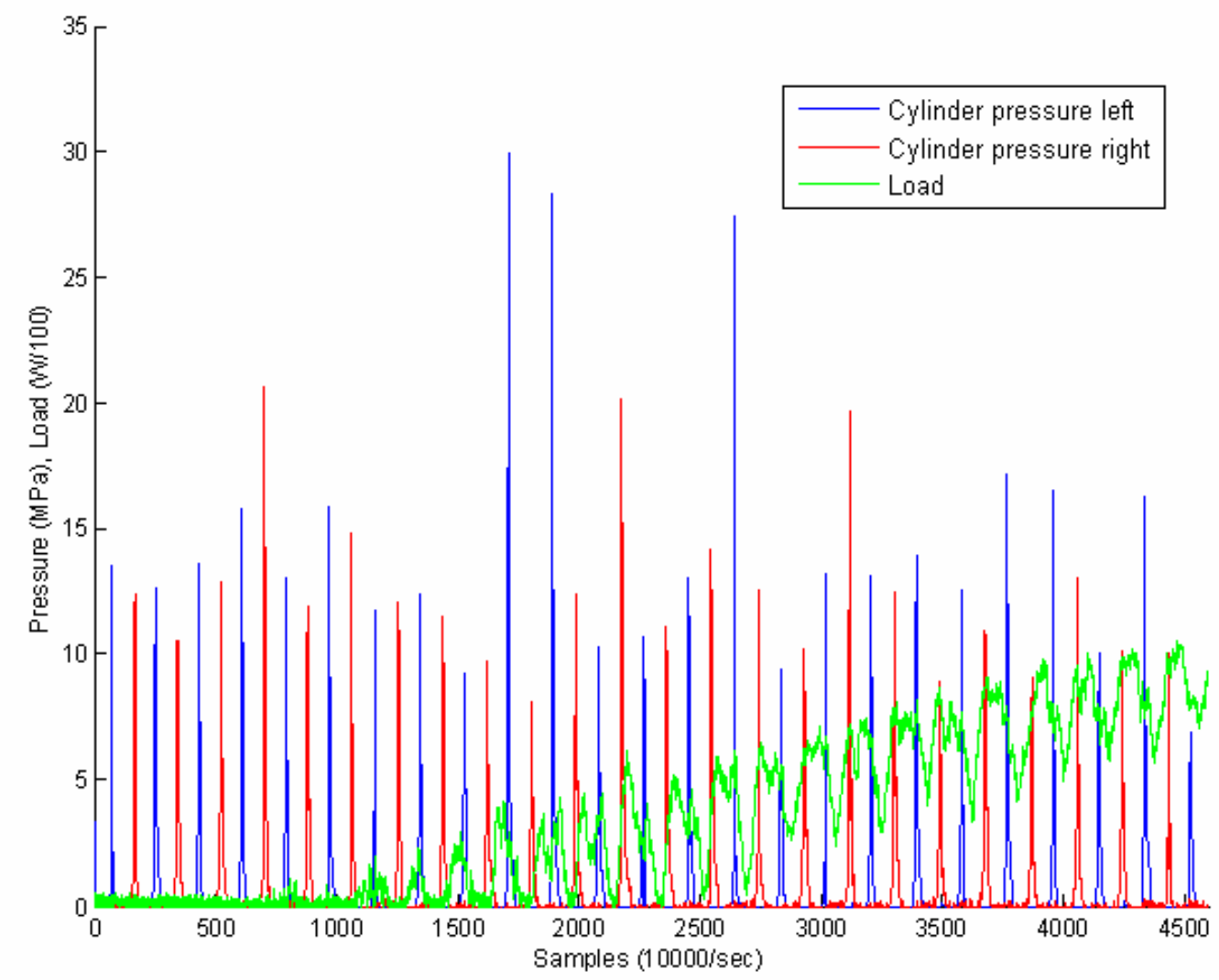

Figure 64. Cylinder pressure and load vs. time. The variation in peak cylinder pressure was so high that it made the effect of load unnoticeable. (Experimental results. Run \# 16 in Table 3)

\subsection{Effect of fuel amount injected}

The fuel amount injected into the cylinder was varied from low (3.7l $\mu \mathrm{l} /$ injection) to high $(17 \mu \mathrm{l} /$ injection). Stochiometric fuel to air ratio was reached with 12.2 $\mu l / i n j e c t i o n$ fuel. The extra fuel at high fueling rate was needed to start the engine at ambient charging pressure.

It was expected that the increased fueling rate would increase the operating frequency and the power output. However, the variation was inconsistent and did not allow conclusions to be drawn. 
Table 7. The effect of fuel amount injected on operating frequency, stalling frequency, output power and efficiency for different injection timing positions.

\begin{tabular}{|c|c|c|c|c|c|}
\hline Fuel amout injected & Injection position & Operating frequency & Stalling frequency & Power & Efficiency \\
\hline (mm/injection) & average (mm before TDC) & $(\mathrm{Hz})$ & $(\mathrm{Hz})$ & $(\mathrm{W})$ & $(\%)$ \\
\hline \multirow{5}{*}{} & 19.75 & 53 & 51 & 520 & 1.25 \\
\cline { 2 - 6 } & 13.5 & 53 & 49 & 700 & 1.75 \\
\cline { 2 - 6 } & 11.5 & 54 & 51 & 1000 & 2.40 \\
\cline { 2 - 6 } & 10.25 & 50 & 47 & 700 & 1.83 \\
\cline { 2 - 7 } & 10.75 & 49 & 47 & 1200 & 3.13 \\
\cline { 2 - 7 } & 9 & 49 & 48 & 325 & 0.83 \\
\cline { 2 - 7 } & 3.5 & 55 & 53 & 2000 & 4.62 \\
\hline \multirow{5}{*}{0.0037} & 14.5 & 52 & 47 & 740 & 8.87 \\
\cline { 2 - 7 } & 14.5 & 50 & 50 & 400 & 4.50 \\
\cline { 2 - 7 } & 10.5 & 51 & 51 & 60 & 0.66 \\
\cline { 2 - 7 } & 8.5 & 50 & 54 & 0 & 0.00 \\
\cline { 2 - 6 } & 7 & 54 & & & \\
\hline
\end{tabular}

\subsection{Peak cylinder pressure analysis}

Peak cylinder pressure was analyzed in attempt to find explanation for the high cycle-to-cycle variation in the linear engine’s behavior.

\subsubsection{Statistical analysis}

Table 8 summarizes the statistical investigation for the maximum cylinder pressure with spark as well as after spark was turned off for different injection timings. Figure 65 aids the visualization of the same data by showing the mean, standard deviation, and coefficient of variant of cylinder pressure with and without spark. 
Table 8. Statistics of the maximum cylinder pressure for different injection timings for the overcharged case.

\begin{tabular}{|c|c|c|c|c|c|c|}
\hline \multirow{2}{*}{ Injection position } & \multicolumn{5}{|c|}{ Peak cylinder pressure (MPa) } \\
\cline { 2 - 7 } (mm before TDC) & \multicolumn{3}{|c|}{ Without spark } & \multicolumn{3}{c|}{ With spark } \\
\cline { 2 - 7 } & Mean w/o spark & STD w/o spark & CV w/o spark & Mean w/ spark & STD w/ spark & CV w/ spark \\
\hline 20 & 14.69 & 5.63 & 38.3 & 15.66 & 8.57 & 54.7 \\
\hline 22 & 14.84 & 5.38 & 36.2 & 18.06 & 6.30 & 34.8 \\
\hline 16 & 13.40 & 3.36 & 25 & 13.58 & 6.96 & 51.3 \\
\hline 14 & 14.13 & 5.61 & 39.6 & 14.22 & 7.76 & 54.6 \\
\hline 8 & 13.49 & 4.41 & 32.7 & 19.02 & 3.17 & 16.6 \\
\hline 8 & 15.09 & 4.41 & 29.2 & 18.93 & 4.03 & 21.3 \\
\hline 4 & 15.96 & 4.43 & 27.7 & 17.93 & 3.52 & 19.8 \\
\hline 0 & 16.57 & 5.14 & 31 & 17.63 & 4.23 & 24 \\
\hline
\end{tabular}

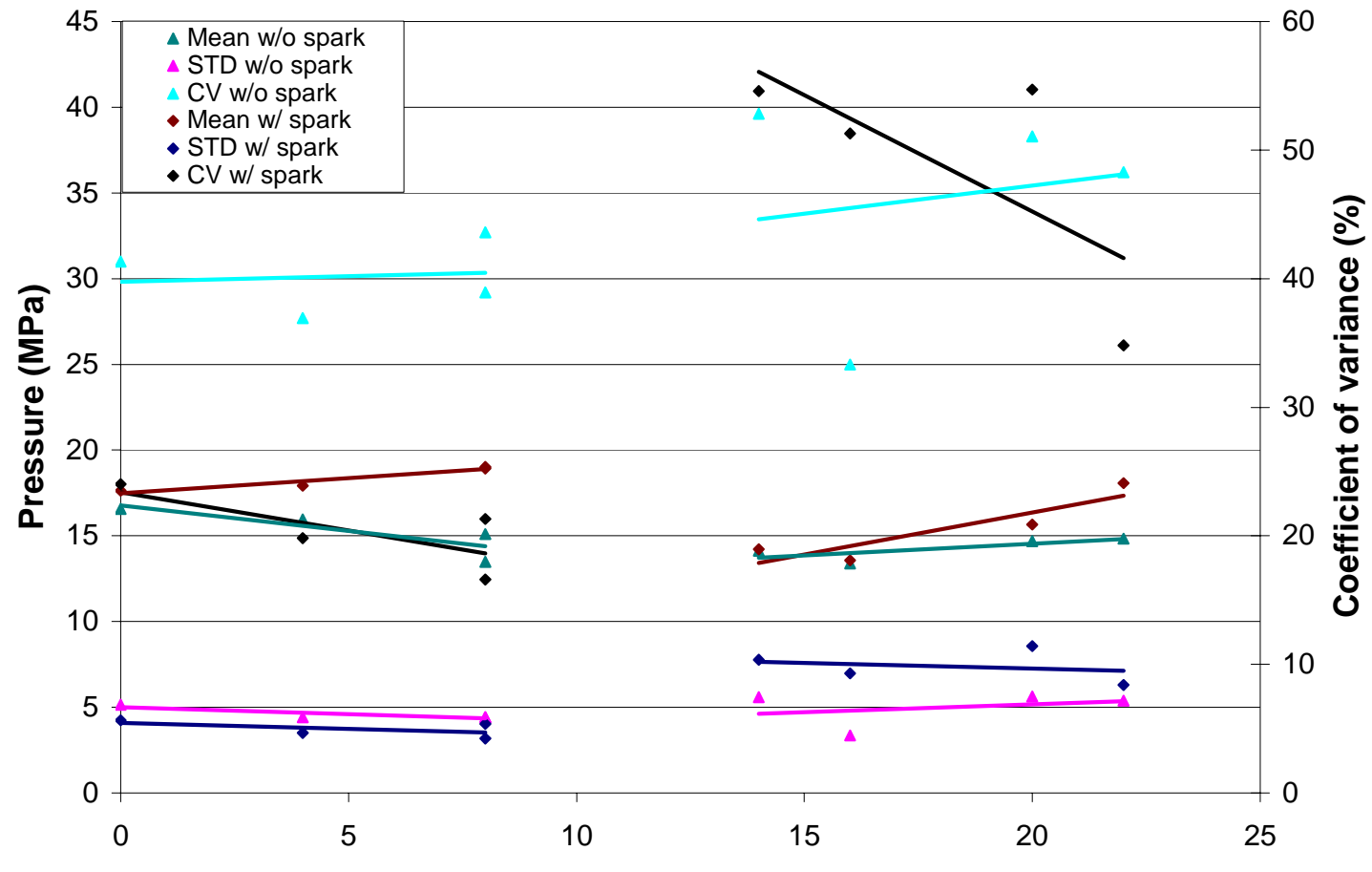

Injection timing $(\mathrm{mm})$

Figure 65. Statistics of the peak pressure analysis. Mean, standard deviation, and coefficient of variant of cylinder pressure with and without spark. Data points between 0 and $8 \mathrm{~mm}$ of injection timing show the left cylinder and data points between 14 and 20 show the right cylinder operation.

While the pressure data were taken, the input value of the mean to the controller was offset by about $12 \mathrm{~mm}$. Thus data points between 0 and $8 \mathrm{~mm}$ of injection timing show the left cylinder and data points between 14 and 20 show the right cylinder 
operation. Therefore in Figure 65 the two sides are divided and trend lines are laid upon the data points separately to show the trends in the different cylinders. Figure 66 and Figure 67 show the same statistical data, mean, standard deviation, and coefficient of variance without and with spark respectively. In Figure 66 and Figure 67 the common trend lines are laid upon the data points of the left and right cylinders.

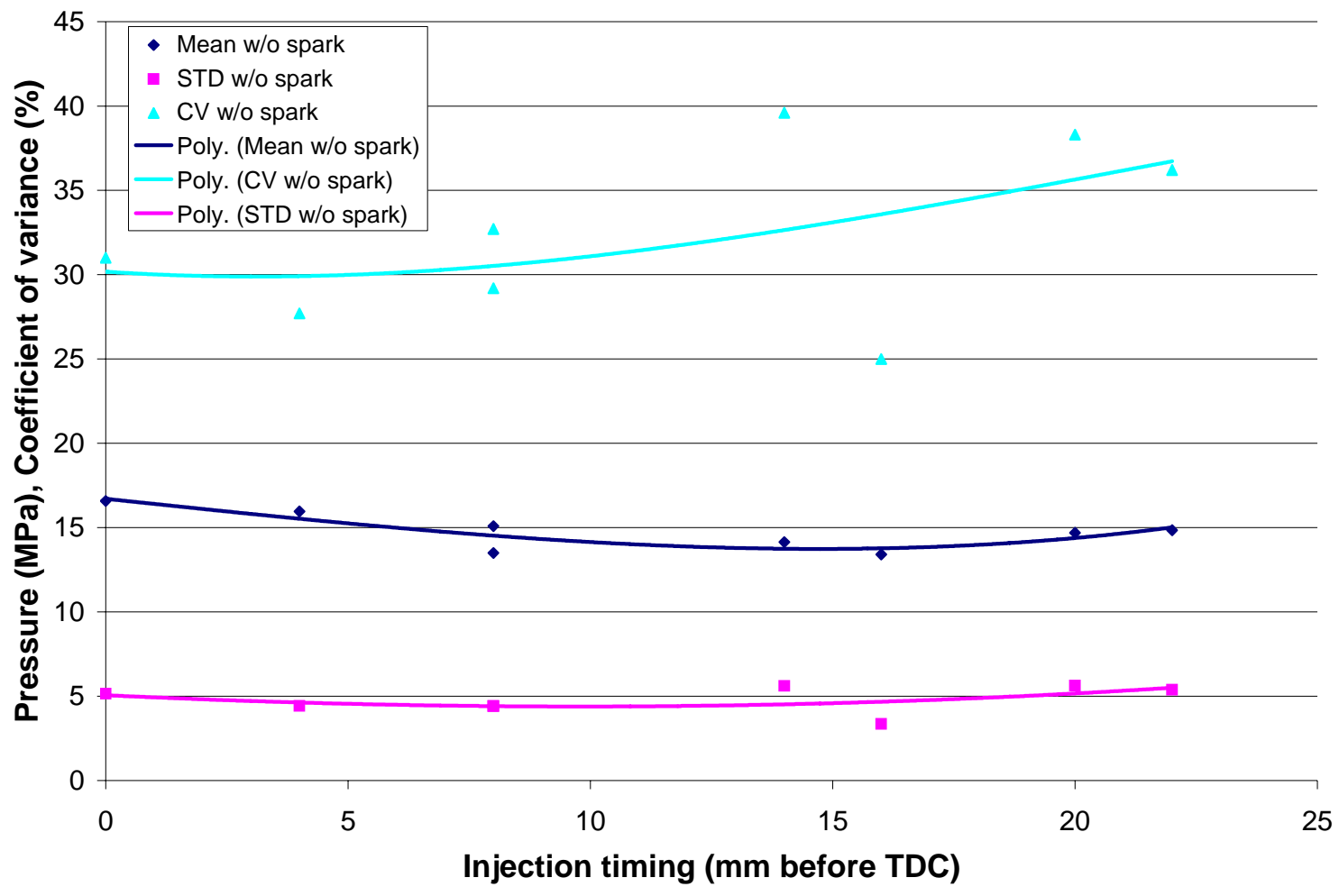

Figure 66. Mean, standard deviation, coefficient of variance of peak cylinder pressure vs. injection timing for engine operation without spark. Data are the same as presented in Figure 65. 


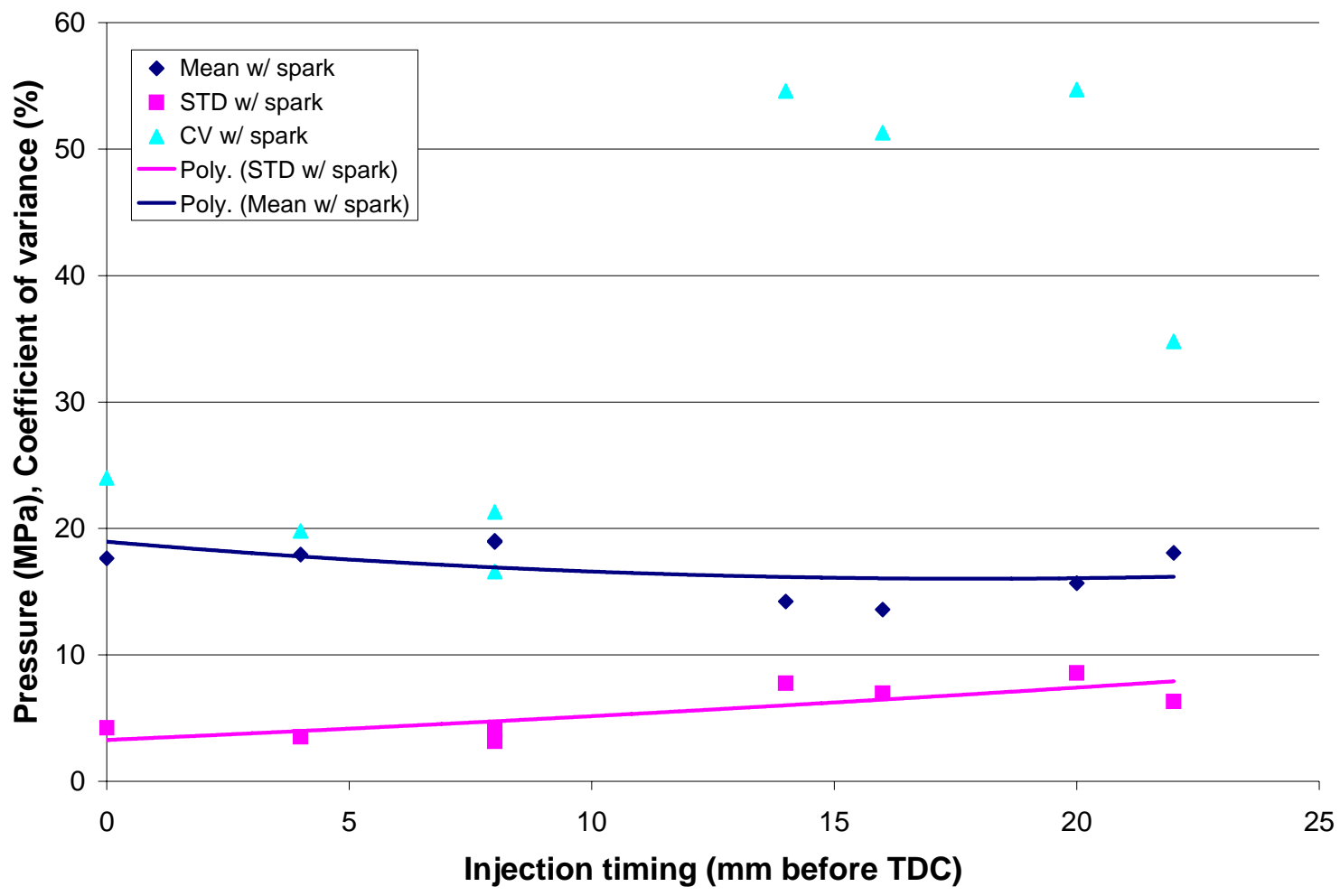

Figure 67. Mean, standard deviation, coefficient of variance of peak cylinder pressure vs. injection timing for engine operation with the sparking aid on. Data are the same as presented in Figure 65.

The trends of the data agree for the separate and the common trend lines, except for the coefficient of variant, for the case when spark was still on. The CV tendency has a decreasing nature in both cylinders but there is a discrepancy in level between the data from the two cylinders. This was clearly the result of the misfire that was caused by the early spark. This allowed the conclusion that when the engine was running with the spark on, the spark had a disturbing effect on the engine behavior.

The mean showed an agreeing trend for both cases with and without spark. The data showed a relatively high value for both compression ignition (close to TDC injection) and pseudo-HCCI (early injection) with a somewhat lowered value in the gray area in between. This phenomenon was believed to be due to the fact that the injection 
close to TDC resulted in higher compression ratio and higher peak pressure than those of early injection. Late injection (close to TDC) resulted in a slow pressure rise that let the translator travel farther towards the cylinder head resulting a higher compression ratio and higher peak pressure when the translator reached TDC. On the other hand, early injection left time for more thorough mixing and evaporation of the fuel that caused fast burning resulting in higher peak cylinder pressure. The injection timing in between resulted in decreased peak pressures that can be explained by extended burning time compared to early injection and lowered compression ratio compared to late injection. Explanation follows in Section 8.4.

\subsubsection{Peak pressure vs. maximum position}

Because the cycle-to-cycle variation was high, peak pressure was compared to maximum piston position to analyze the connection between those two variables. Figure 68 shows the peak pressure and maximum piston position in each cycle. Figure 69 shows normalized peak pressure vs. normalized compression. There was a very low correlation between peak pressure and maximum position with a correlation coefficient of $\mathrm{R}^{2}=0.0203$. This high cycle-to-cycle variation was believed to be due partially to variation in injector behavior caused by vibration and partially to the inaccuracy of measuring pressure and position. 


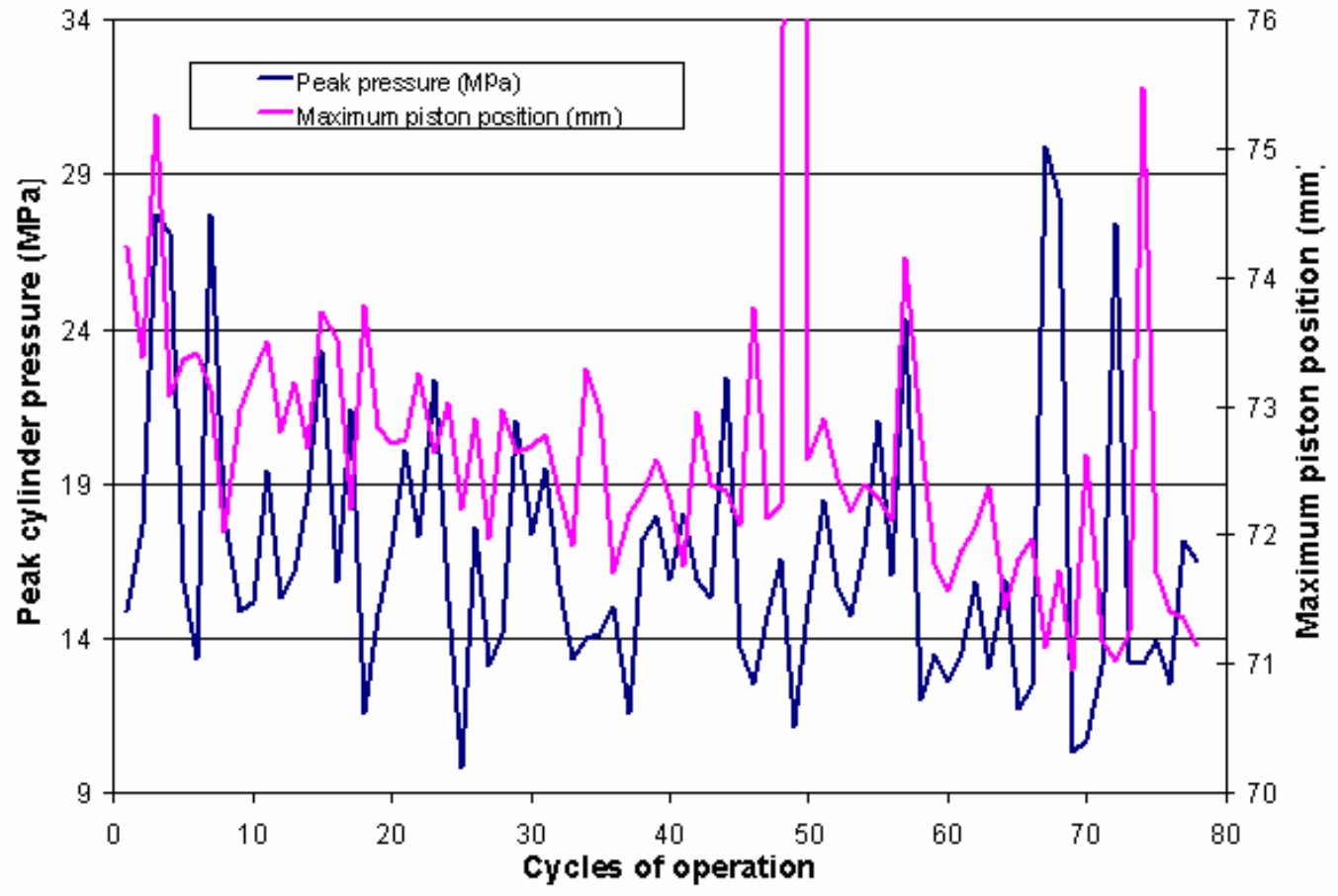

Figure 68. Peak cylinder pressure and maximum piston position.

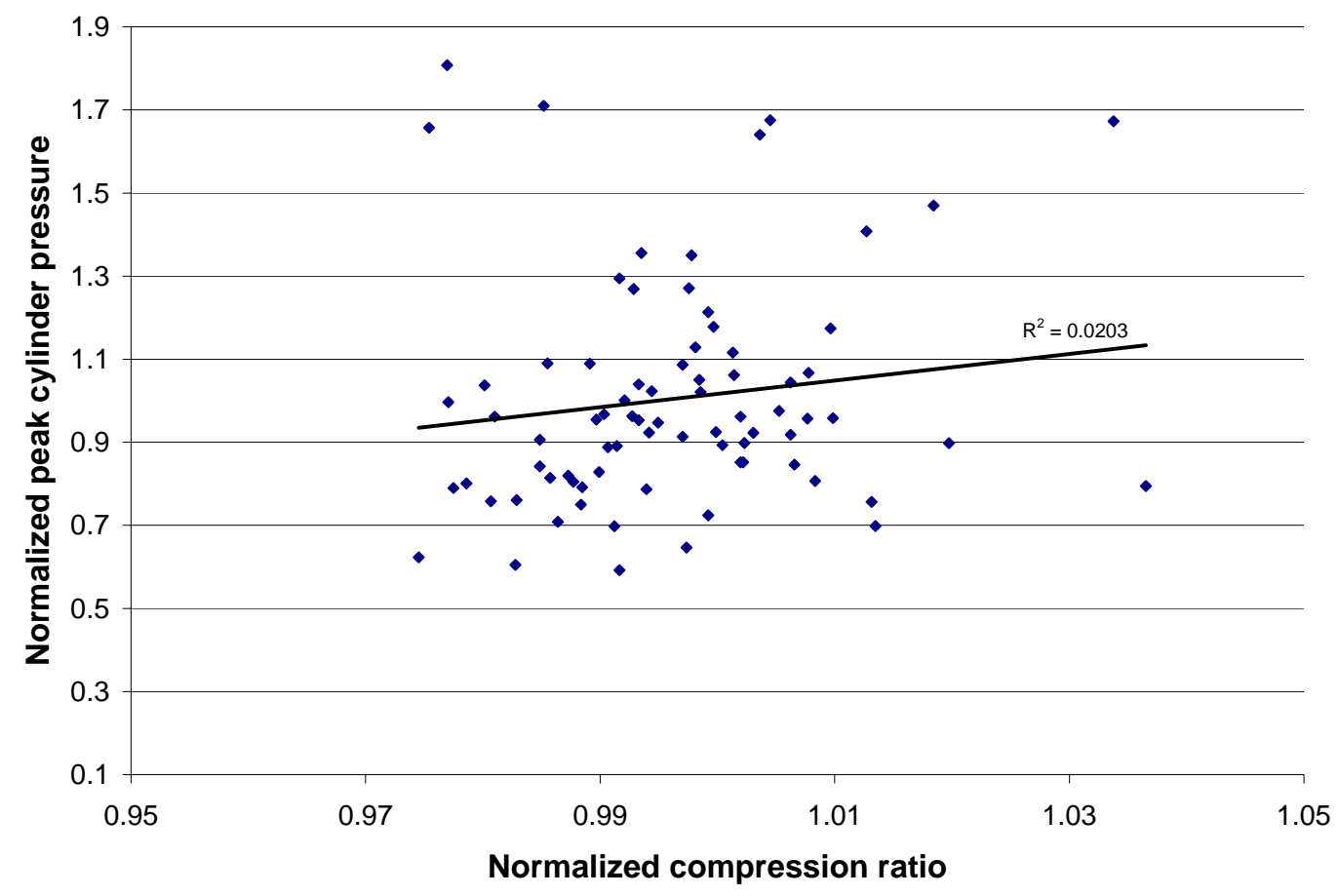

Figure 69. Normalized peak cylinder pressure vs. normalized compression ratio. 


\subsubsection{Pressure-position diagram}

Time alignment of the gathered data was accomplished by lining up the position data and the pressure data. For this purpose, the final section of Run \# 17 in Table 3 was used. In Run \# 17, the injection timing was continually retarded until the engine stalled. After the engine stalled and no combustion occurred the engine went through a ring down process before it came to a complete stop. This ring down section of Run \# 17 was used to perform the time alignment of the pressure and position data. The pressure measuring system was claimed to have no time delay [121]. The position sensor was claimed to have $80 \mu$ s delay [122]. However, pressure and position lined up when position data were delayed by $0.7 \mathrm{~ms}$. Figure 70 and Figure 71 show the pressure-position diagram for the ring down before and after time alignment respectively.

Pressure-position diagrams are shown in Figure 72 through Figure 75 from early injection (pseudo-HCCI operation) to injection at TDC (DICI operation). 


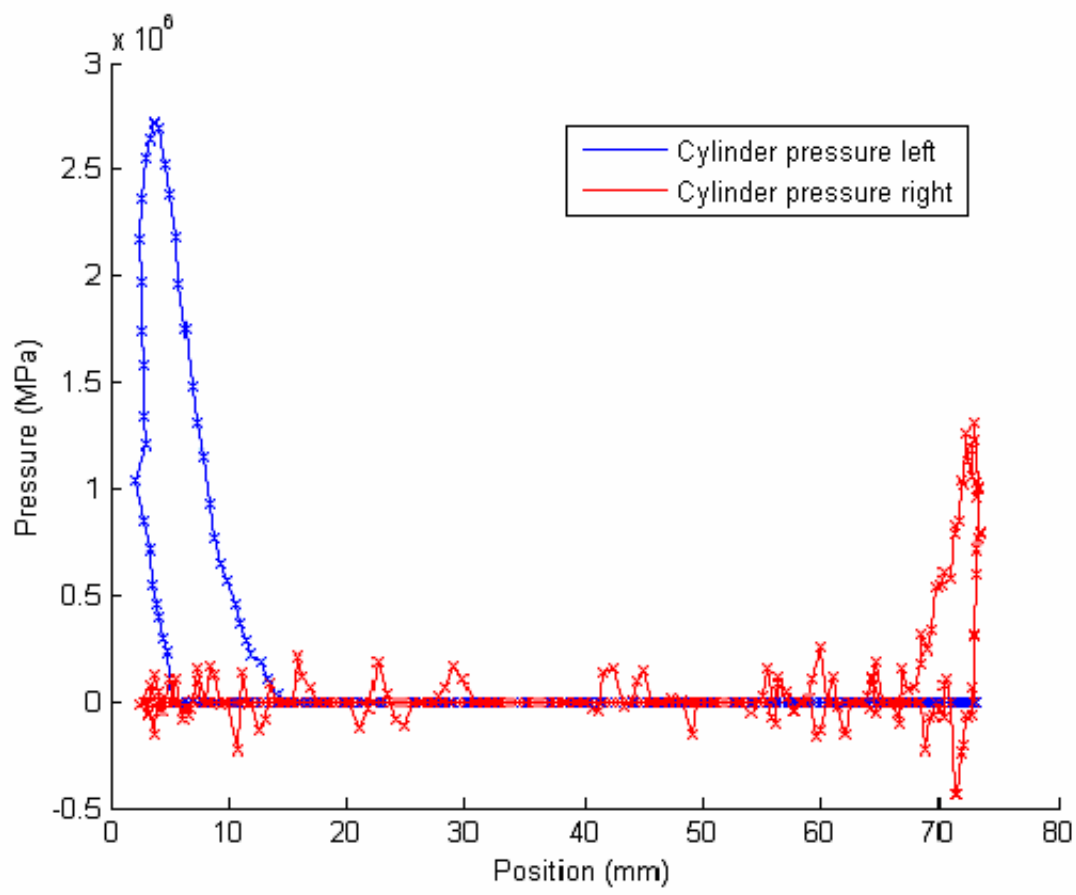

Figure 70. Pressure-position diagram before time alignment. It was for the ring down period.

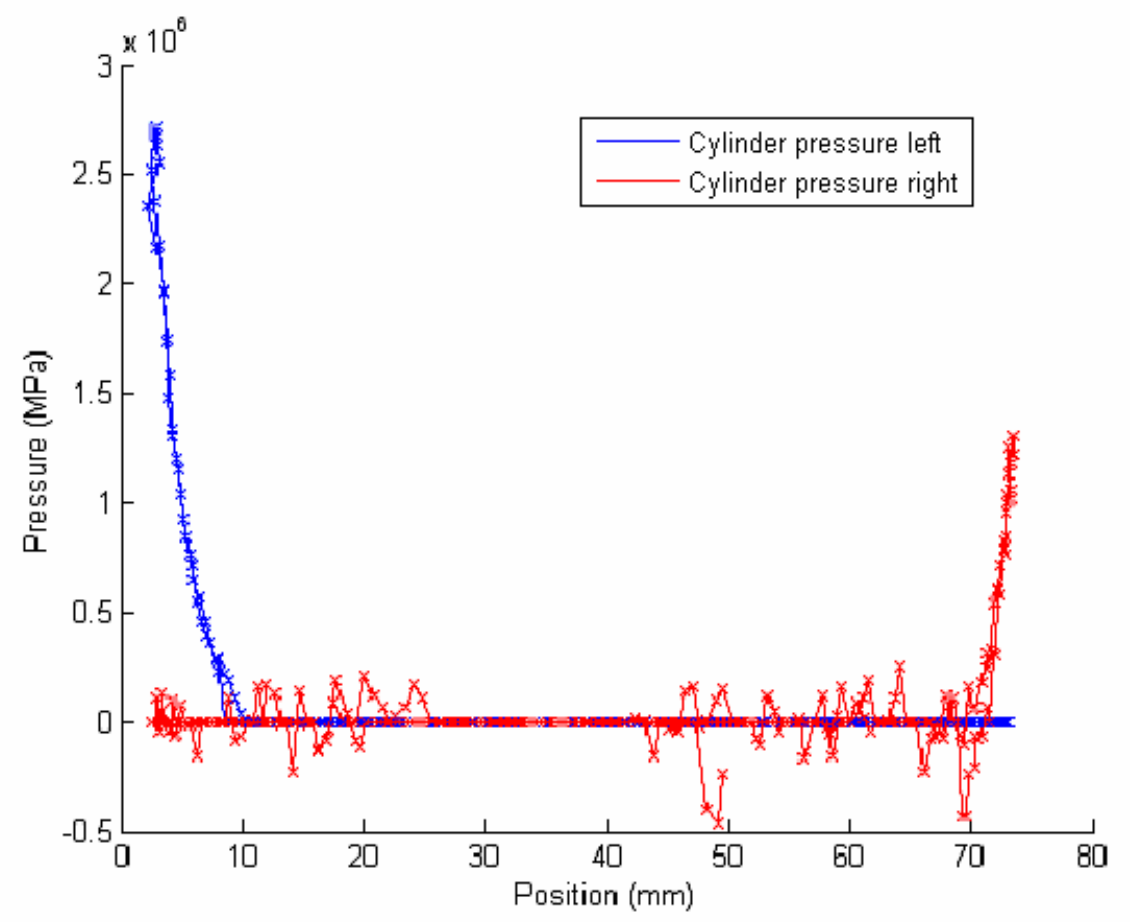

Figure 71. Pressure-position diagram for the engine ringing down period. Position data were delayed by $0.7 \mathrm{~ms}$. Data are the same as in Figure 70. 


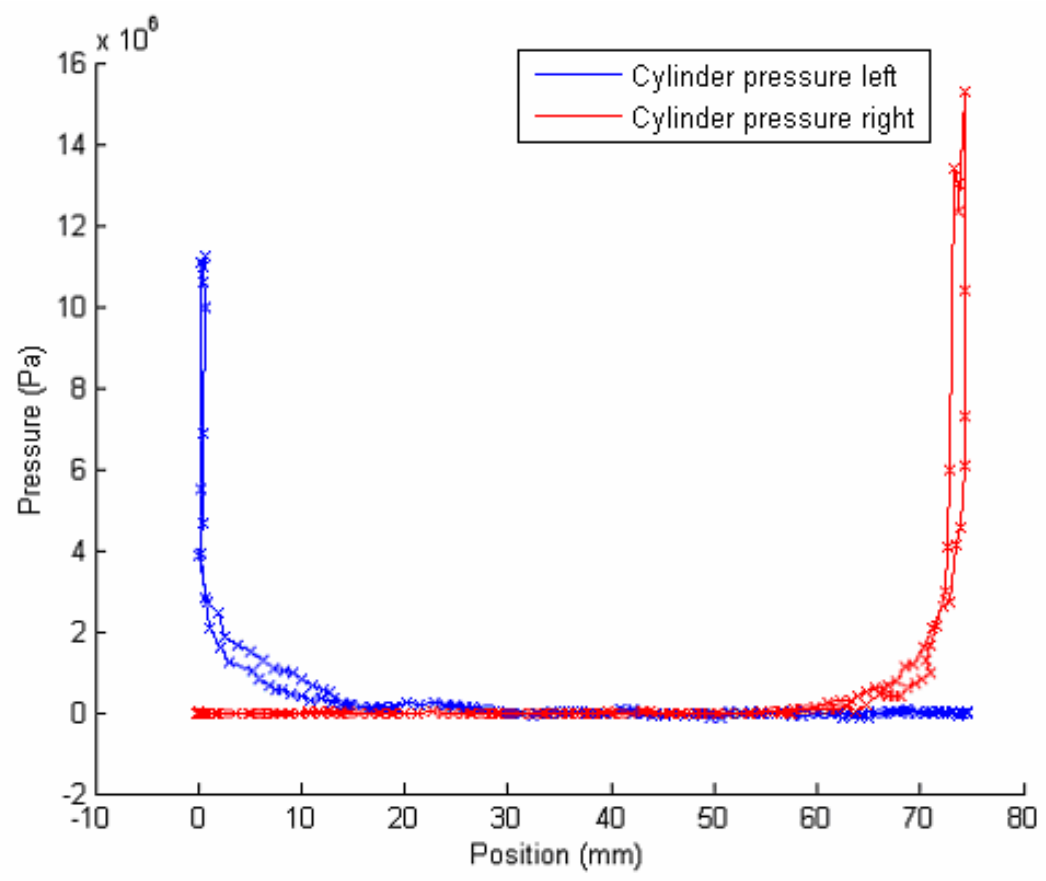

Figure 72. Pressure-position diagram for Run \# 14 in Table 3. Fueling rate was 3.7 $\mu \mathrm{l} /$ injection. Injection timing was 8 and $20 \mathrm{~mm}$ on the left (blue) and the right (red) side respectively.

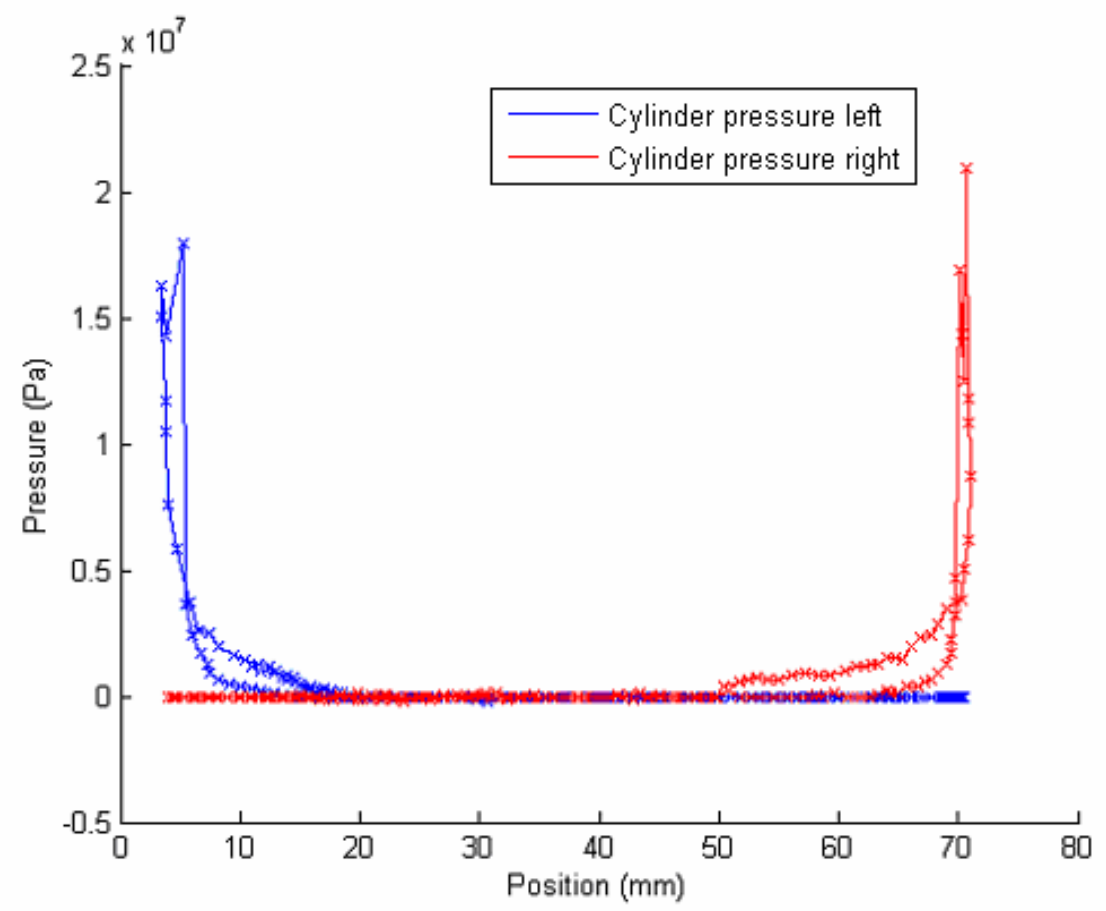

Figure 73. Pressure-position diagram for Run \# 16 in Table 3. Fueling rate was 3.71 $\mu \mathrm{l} /$ injection. Injection timing was 4 and $16 \mathrm{~mm}$ on the left (blue) and the right (red) side respectively. No load. 


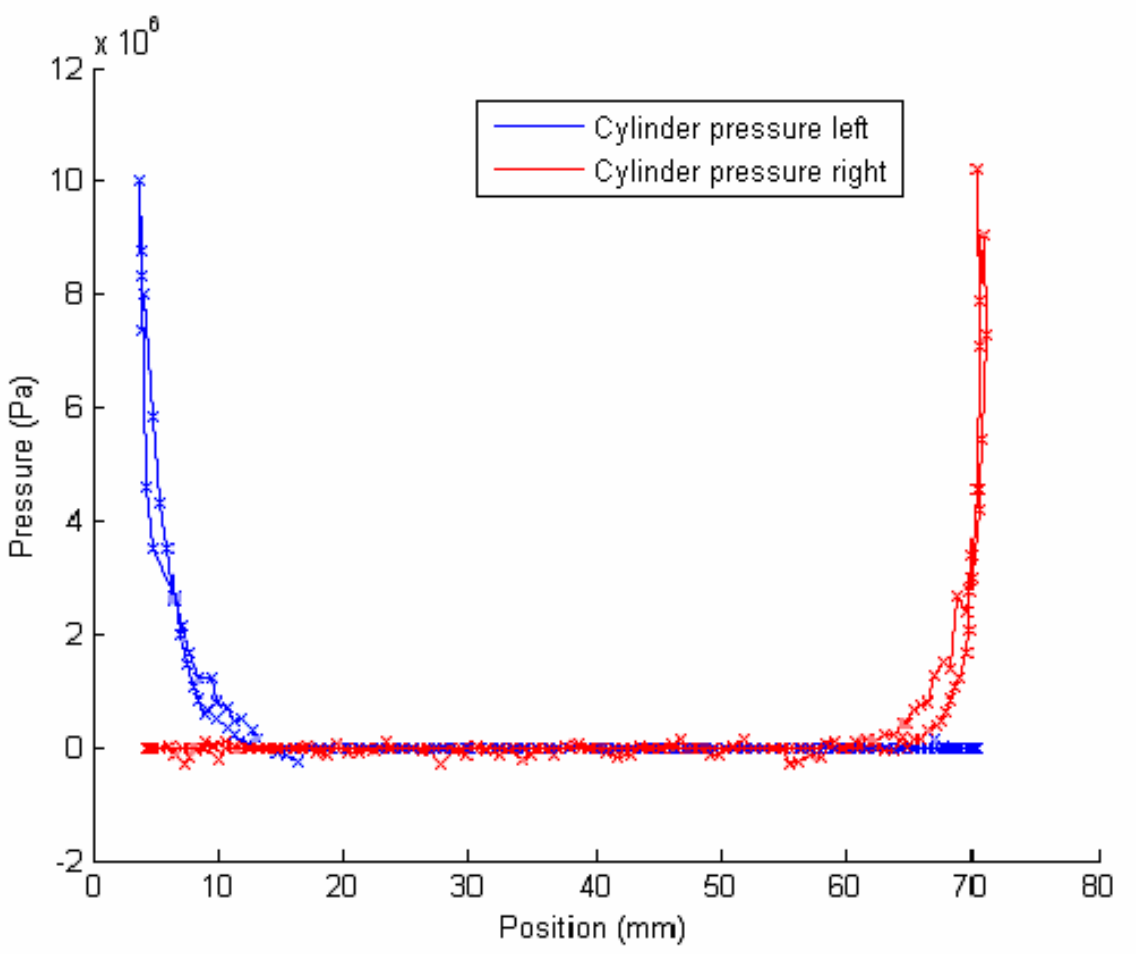

Figure 74. Pressure-position diagram for Run \# 16 in Table 3. Fueling rate was 3.71 $\mu \mathrm{l} /$ injection. Injection timing was 4 and $16 \mathrm{~mm}$ on the left (blue) and the right (red) side respectively. Load was applied until the engine stalled.

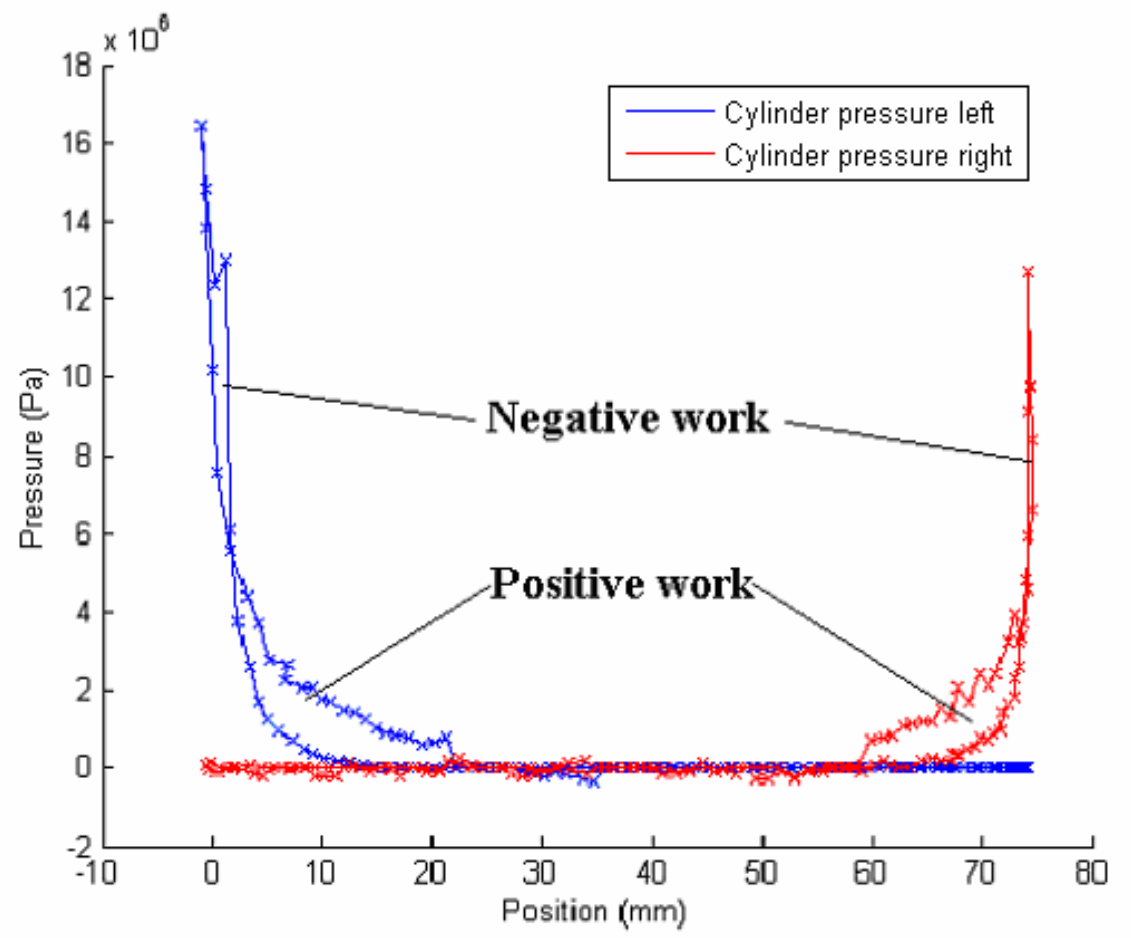

Figure 75. Pressure-position diagram for Run \# 17 in Table 3. Fueling rate was 3.71 $\mu \mathrm{l} /$ injection. Injection timing was 0 and $14 \mathrm{~mm}$ on the left (blue) and the right (red) side respectively. 
Not much difference can be seen between the individual pressure-position diagrams. They are all in the same range with high cycle-to-cycle variation. As Young [120] summarized it, the high cycle-to-cycle variation is not unusual in internal combustion engines. The interesting phenomenon that can be seen in the pressureposition diagrams is the negative work that occurred. Negative and positive work were indicated in Figure 75. Negative work occurred when the engine was over fueled for the given load. Figure 73 shows the engine with no load and Figure 74 shows it with load before the engine stalled. It can be seen that negative work was more substantial in the case of no load than it was for the high load. The negative work was used to stop and return the translator. Work was wasted in the form of lost heat. In the case when high load was applied the load was used to stop the translator, which eliminated the negative work. Careful control strategy will be necessary for the linear engine to avoid overfueling that caused the negative work.

The question was: were these negative work regions true or they were just artifacts of the pressure and position measuring equipment. Pressure-position diagrams were plotted for the simulation results to clarify the situation. Figure 76 and Figure 77 show pressure-position diagrams for the simulation results. For better visibility, Figure 77 shows a magnified segment of the pressure-position diagram in Figure 76. The negative work regions were clearly visible providing support for the explanations above. It was also clear that the negative work disappeared as load was applied to the engine. For this particular run, the bore was: $108.5 \mathrm{~mm}$, effective stroke: $27.1 \mathrm{~mm}$, free travel: $13.57 \mathrm{~mm}$, mass: $1.9602 \mathrm{~kg}$, fuel injected: $8.9 \mu \mathrm{l}$, injection timing: $24.4 \mathrm{~mm}$, ignition 
timing: $10.3 \mathrm{~mm}$. Figure 76 and Figure 77 show operation cycles for 25, 35, and $45 \mathrm{~kW}$ of applied load. The engine simulation stalled at $46 \mathrm{~kW}$.

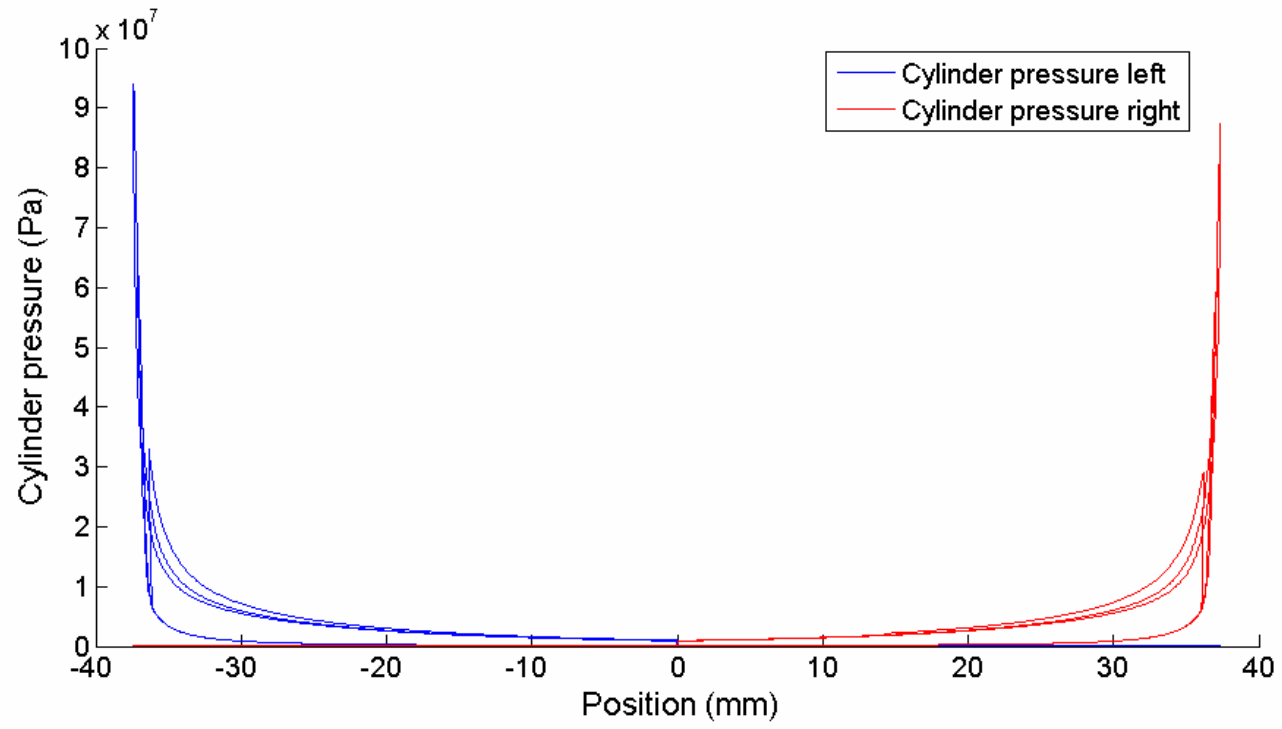

Figure 76. Pressure-position diagram for the simulation results.

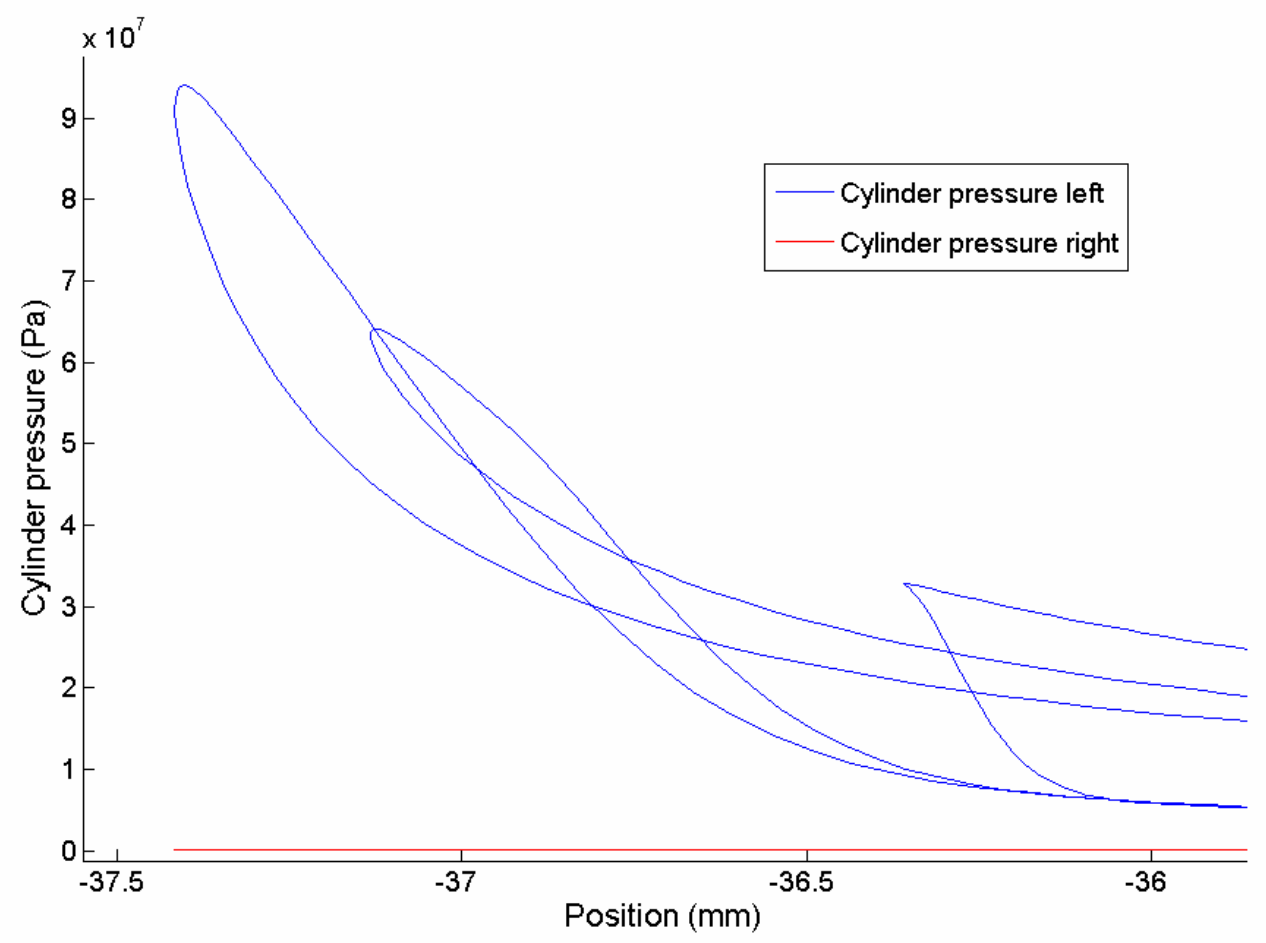

Figure 77. A magnified segment of the pressure position diagram in Figure 76. 
Another interesting phenomenon was the double pressure peak that occurred in the diagrams. The double peak was present for about $50 \%$ of all combustions. It indicates that there is a premix burn and a diffusion burn happening even in the case of early injection. This was believed to be due partially to noise and the heat effect in the pressure measuring equipment.

\subsection{Heat release rate}

The heat release rate was calculated for simulation and experimental data and they were compared. Figure 78 shows the heat release rate for both the simulation and experiment. They look similar, however, it is not surprising that they do not match exactly. The difference is due to two factors. The crudeness of the simulation model and the inaccuracy of the pressure measurement.

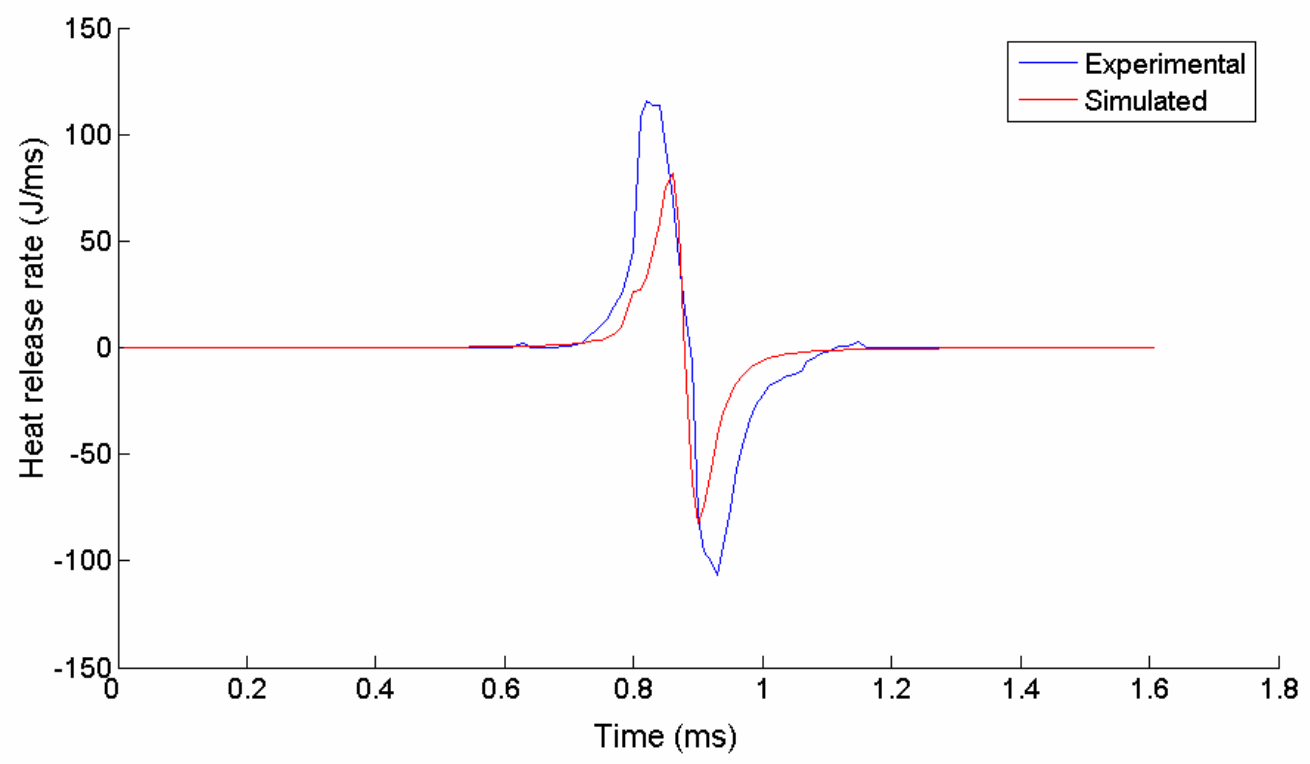

Figure 78. Heat release rate for simulation (red) and experimental (blue) data. 


\subsection{Conclusions}

Experimental results of the linear engine were presented in this chapter. The effect of translator mass, stroke length, overcharging, fueling rate, injection timing, and load were presented in this study. Increasing the translator mass resulted in decreased operating frequency. Increasing the stroke length also resulted in decreased operating frequency. Overcharging and increased fueling rate, both, resulted in increased power output, efficiency, and operating frequency. Advancing injection timing resulted in increased frequency, efficiency and power output, but decreased stalling frequency. Injection timing had an unexpected effect on peak cylinder pressure. Advancing the injection timing started to decrease the peak cylinder pressure. However, with further advancing the injection timing pressure increased. This called for further investigation by simulation presented in Chapter 8 . The cylinder pressure analysis revealed that the engine operation had regions that resulted in negative work. This was due to over fueling the engine. The energy of the extra fuel was wasted on stopping and returning the translator. In the case of proper fueling rate the translator would be stopped by the load force not by the compression force, not by the force created by combustion pressure. 


\section{Comparing simulation and experimental data}

Simulation was performed with a double goal. One was to see if trends in the experimental data matched the trends in the model. The other was to expand on the area where experimental results were lacking. In this chapter, experimental and simulation data were compared.

\subsection{Effect of moving mass}

The effect of the moving mass was investigated on operating frequency, peak cylinder pressure, and compression ratio. Table 9 and Figure 79 show the results of the study on the effect of the mass. Fuel injected was $40 \mu \mathrm{l} /$ injection at $10 \mathrm{~mm}$ before TDC.

Table 9. The effect of the moving mass on operating frequency, peak cylinder pressure, and compression ratio.

\begin{tabular}{|c|c|c|c|c|c|}
\hline $\begin{array}{c}\text { Mass } \\
(\mathrm{kg})\end{array}$ & $\begin{array}{c}\text { Simulated } \\
\text { frequency }(\mathrm{Hz})\end{array}$ & $\begin{array}{c}\text { Measured } \\
\text { frequency }(\mathrm{Hz})\end{array}$ & $\begin{array}{c}\text { Max. position } \\
(\mathrm{mm})\end{array}$ & $\begin{array}{c}\text { Peak pressure } \\
(\mathrm{MPa})\end{array}$ & $\begin{array}{c}\text { Compression } \\
\text { ratio }\end{array}$ \\
\hline 0.8 & 73.47 & $\mathrm{n} / \mathrm{a}$ & 35.1 & 4.05 & 13.1 \\
\hline 1 & 71.8 & $\mathrm{n} / \mathrm{a}$ & 35.86 & 5.88 & 17.76 \\
\hline 2 & 62.8 & $\mathrm{n} / \mathrm{a}$ & 36.6 & 11.8 & 27.14 \\
\hline 2.8 & 57.8 & 50 & 37 & 16.9 & 38 \\
\hline 4 & 52.3 & $\mathrm{n} / \mathrm{a}$ & 37.26 & 25.3 & 51.35 \\
\hline 4.7 & 49.8 & 35 & 37.35 & 29.6 & 58.46 \\
\hline 6 & 46.3 & $\mathrm{n} / \mathrm{a}$ & 37.42 & 40.4 & 74.51 \\
\hline 8 & 42 & $\mathrm{n} / \mathrm{a}$ & 37.57 & 51.2 & 90.48 \\
\hline
\end{tabular}




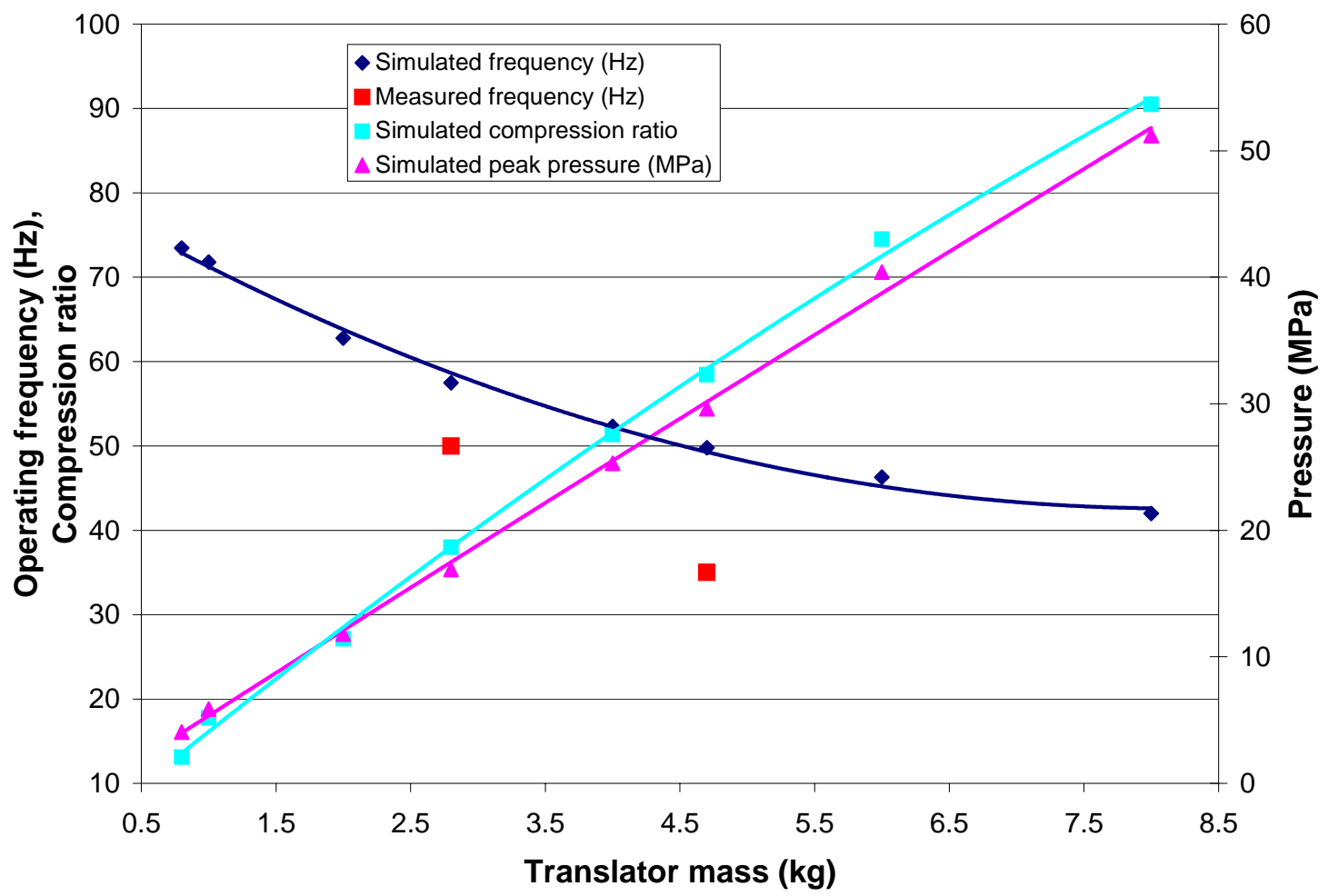

Figure 79. The effect of the moving mass on operating frequency, peak cylinder pressure, and compression ratio. Fuel amount injected was $40 \mu \mathrm{l} /$ injection at $10 \mathrm{~mm}$ before TDC.

The decreasing translator mass resulted in decreased peak cylinder pressure and decreased compression ratio. As translator mass was decreased the engine was unable to operate at $0.7 \mathrm{~kg}$ because the compression ratio dropped below the critical value so combustion was not sustainable. This was due to the fact that when combustion happened in one cylinder, the inertial energy of the moving mass was not enough to compress the charge in the cylinder on other side to auto ignition. The increasing mass yielded increasing peak cylinder pressure and compression ratio, which improved the engine's ability to sustain operation. At the same time operating frequency decreased. It appears that the translator weight does not have an upper limit from the engine's point of view only from material strength point of view. Thus for a desired power output, the current carrying capability of the alternator stator and material properties of the engine 
components should be the dominant design parameters on the engine's operating frequency, which is largely influenced by the weight of the translator.

\subsection{The effect of stroke length}

Simulation was performed to investigate the effect of stroke length on operating frequency, peak cylinder pressure, and compression ratio. Note should be taken that it was not effective stroke length that was investigated in this case. Effective stroke length was kept constant at $38 \mathrm{~mm}$. A representative of total stroke length is free travel that was defined in Section 7.3. Table 10 and Figure 80 show the results of the simulation. Increasing the free travel decreased the operating frequency and the compression ratio as was expected. As the free travel increased, it took a longer time for the translator to travel from BDC to TDC. This resulted in lowered operating frequency and compression ratio. The surprising phenomenon was that decreased free travel resulted in decreased peak pressure. This needs further investigation for a feasible explanation.

Table 10. The effect of free travel on operating frequency, peak pressure, and compression ratio. Fuel injected was $40 \mu \mathrm{l} /$ injection at $10 \mathrm{~mm}$ before TDC.

\begin{tabular}{|c|c|c|c|c|c|}
\hline $\begin{array}{c}\text { Free travel } \\
(\mathrm{mm})\end{array}$ & $\begin{array}{c}\text { Simulated } \\
\text { frequency }(\mathrm{Hz})\end{array}$ & $\begin{array}{c}\text { Measured } \\
\text { frequency }(\mathrm{Hz})\end{array}$ & $\begin{array}{c}\text { Max. position } \\
(\mathrm{mm})\end{array}$ & $\begin{array}{c}\text { Peak pressure } \\
(\mathrm{MPa})\end{array}$ & $\begin{array}{c}\text { Compression } \\
\text { ratio }\end{array}$ \\
\hline 8 & 57.3 & $\mathrm{n} / \mathrm{a}$ & 40.1 & 20.22 & 22.11 \\
\hline 5 & 57.5 & 48 & 39.45 & 18.83 & 38.57 \\
\hline 3 & 57.6 & $\mathrm{n} / \mathrm{a}$ & 38.4 & 18.12 & 35.91 \\
\hline 0 & 58 & 50 & 37 & 18.22 & 38.00 \\
\hline-3 & 58.7 & $\mathrm{n} / \mathrm{a}$ & 35.6 & 17.5 & 40.56 \\
\hline-5 & 58.9 & $\mathrm{n} / \mathrm{a}$ & 34.7 & 17.3 & 44.38 \\
\hline
\end{tabular}




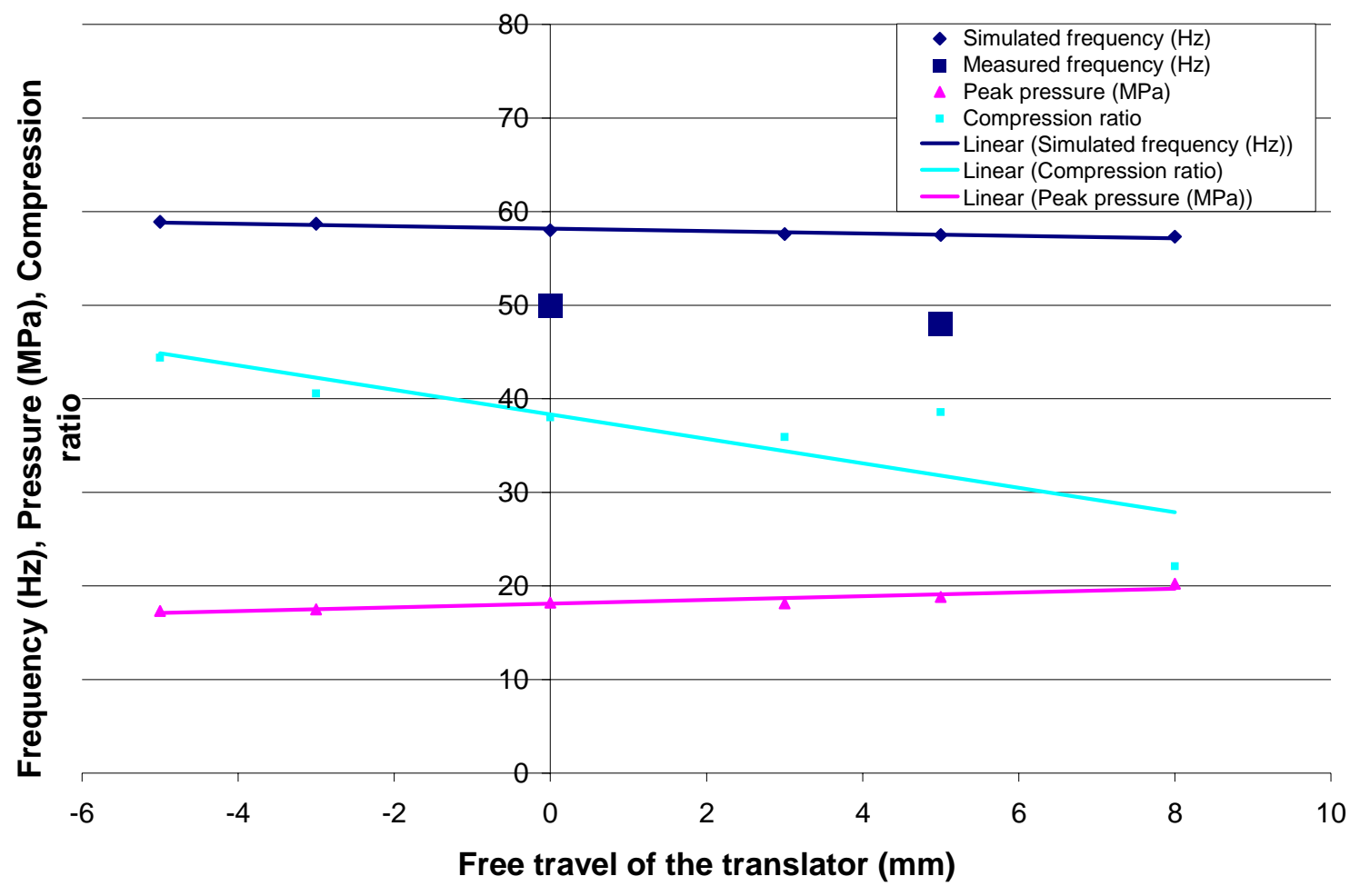

Figure 80. The effect of free travel on operating frequency, peak pressure, and compression ratio. Fuel injected was $40 \mu \mathrm{l} /$ injection at $10 \mathrm{~mm}$ before TDC.

\subsection{The effect of the amount of fuel injected}

The experimental data were not sufficient to examine the effect of the amount of fuel injected on engine behavior. However, the initial testing of the engine suggested that operating frequency was insensitive to the amount of fuel injected into the cylinder. This would have meant that the engine had a narrow window of preferred frequency of operation. Further investigation determined that the engine was controlled by the amount of air rather than the amount of fuel in the cylinder during those initial test runs. The uncertainty about the fuel amount-frequency relationship required the simulation study of the area. For this study the amount of fuel injected into the cylinder was gradually decreased until the engine was unable to sustain operation. Figure 81 shows the effect of 
the amount of fuel injected on frequency. It is obvious that more fuel resulted in higher operating frequency. It was also clear, as it can also be seen in the results in Section 8.1, that an engine with greater translator mass has a better ability to sustain operation. An engine with larger translator mass is able to sustain operation on less fuel than an engine with a lighter translator. In the simulation, an engine with a $2.8 \mathrm{~kg}$ translator was able to operate on as low as $3.5 \mu \mathrm{l} /$ injection, while the engine with a $4.7 \mathrm{~kg}$ translator was able to operate on as little fuel as $2.3 \mu \mathrm{l} /$ injection resulting in $49 \mathrm{~Hz}$ and $35 \mathrm{~Hz}$ respectively. These numbers were around experimental data, $50 \mathrm{~Hz}$ and $35 \mathrm{~Hz}$, which proves that the engine during the first test runs, where much more fuel was injected into the cylinder, was severely under scavenged. The required fuel to create stochiometric air-fuel ratio would be $12.2 \mu \mathrm{l} /$ injection, assuming $100 \%$ volumetric efficiency.

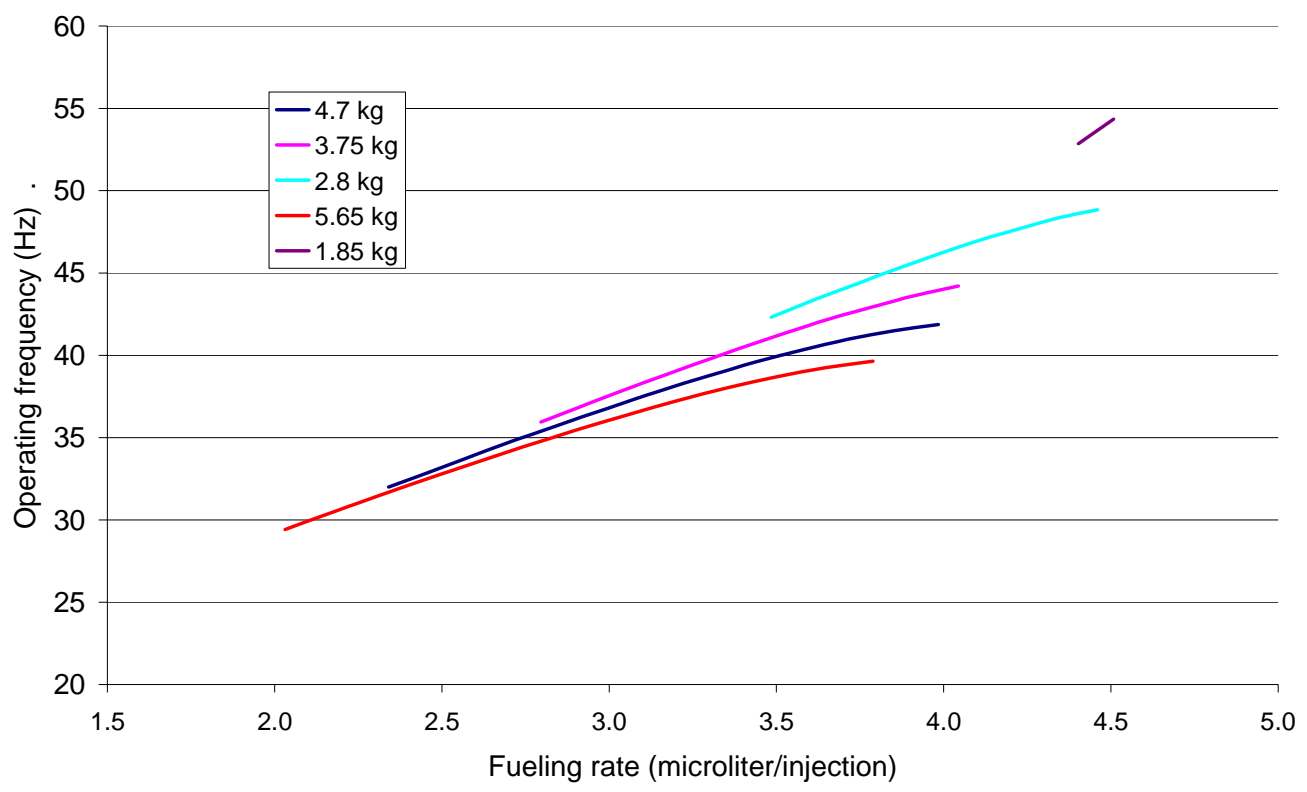

Figure 81. Engine operating frequency vs. amount of fuel injected. No load was applied. Heat release was instantaneous. 
Figure 82 shows the lowest operating frequency vs. translator mass. Fuel amount injected was different for each data point.

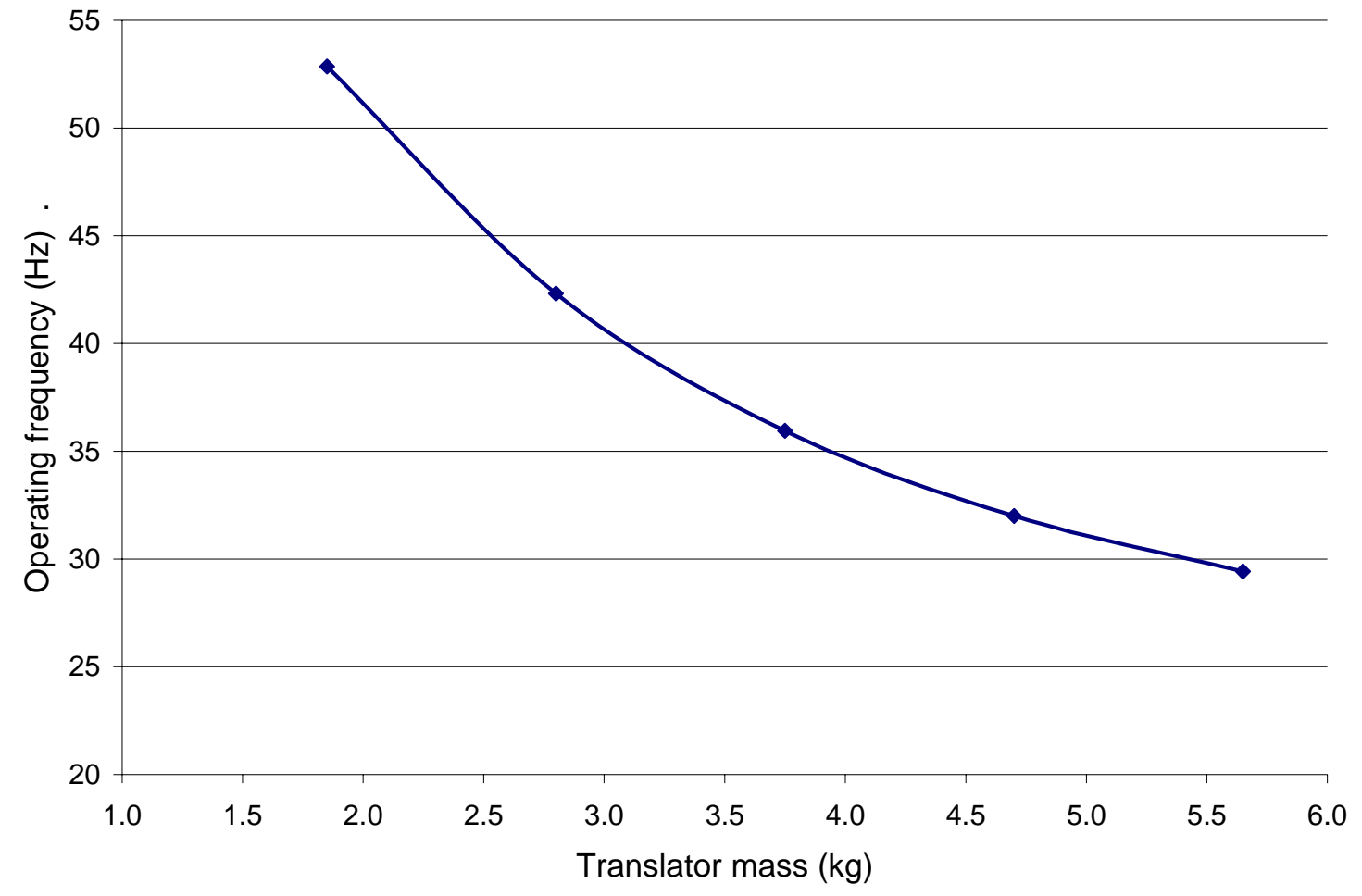

Figure 82. Lowest operating frequency vs. translator mass. Fuel amount injected was different for each data point.

\subsection{The effect of injection timing}

For examining the effect of injection timing, the heat release rate (Wiebe function) was varied as a function of injection timing. The heat release rates used in the simulation for different injection timings can be seen in Figure 83. It is clear that even very early injection would not result in perfect HCCI operation but the aim of this part of the study was to show the benefits of the HCCI operation therefore fuel injection at 38 mm before the head was chosen to represent pseudo-HCCI operation. In the case of early injection the burning happens completely in the premix burn regime. Van Blarigan et al. [74] reported that $95 \%$ of the fuel is burned in $80 \mu \mathrm{s}$. A value of $150 \mu$ s burning time 
was chosen for the pseudo-HCCI operation to compensate for the effect of possible imperfect mixing. The late injection, $3 \mathrm{~mm}$ before the head, represents regular DICI operation with $10 \%$ premix burn in $150 \mu$ s and total burning time of $5 \mathrm{~ms}$. Between the two extreme cases, pseudo-HCCI and DICI, the ratio of the fuel burned in premix burn and the diffusion part of the burn was varied as a linear function of injection timing.

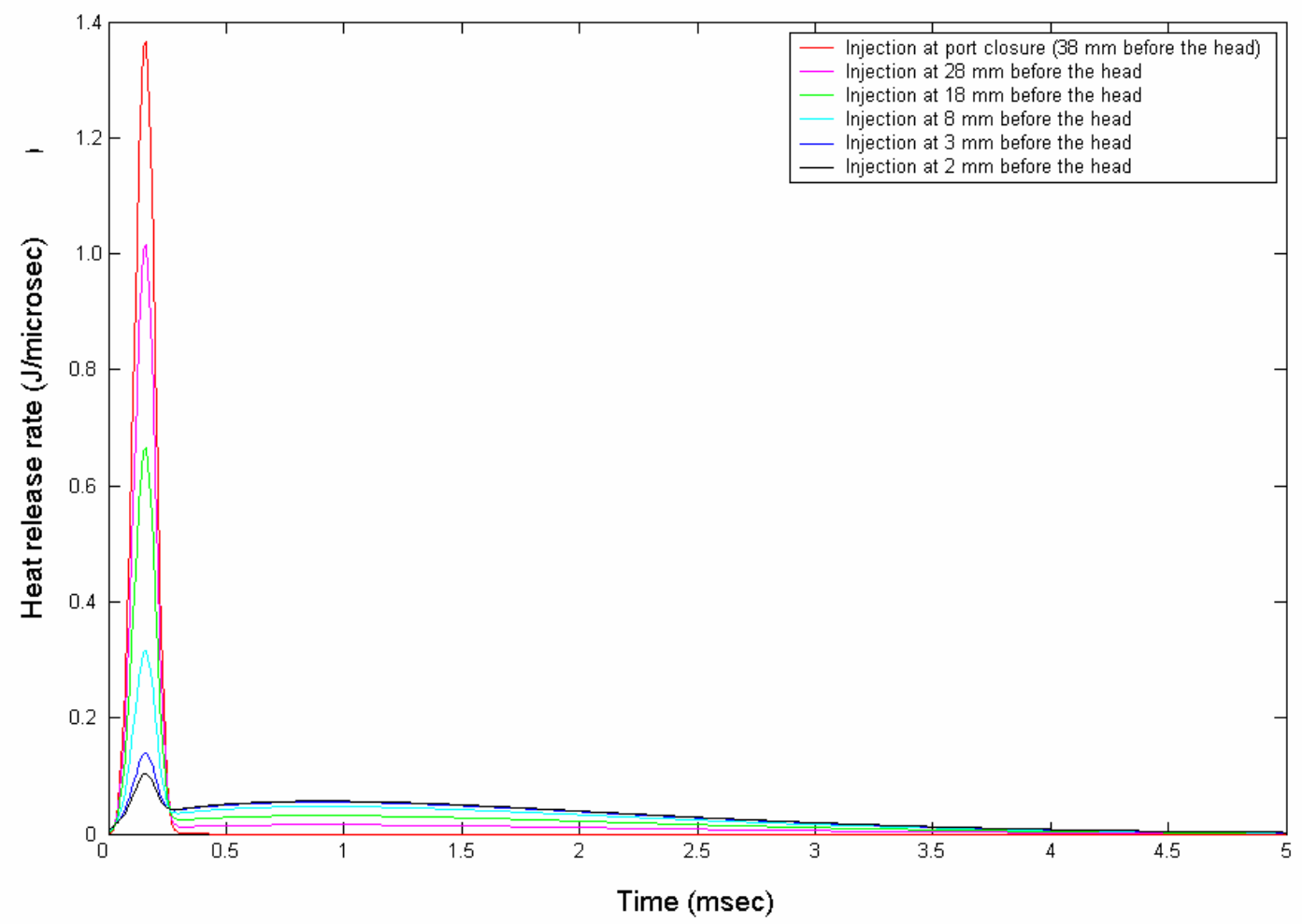

Figure 83. Heat release rates for different injection timings were calculated by using double Wiebe functions.

Injection timing was varied in the simulation model to determine the effect of injection timing on operating frequency, compression ratio, and peak cylinder pressure. The simulation was run with no load and $40 \mu \mathrm{l}$ fuel/injection. The results are summarized in Table 11 and visualized in Figure 84. 
Table 11. The effect of injection timing on operating frequency, compression ratio, and peak cylinder pressure.

\begin{tabular}{|c|c|c|c|c|}
\hline Injection timing $(\mathrm{mm}$ before TDC) & Frequency $(\mathrm{Hz})$ & Max. position $(\mathrm{mm})$ & Peak pressure (MPa) & Compression ratio \\
\hline 38 & 66.1 & 36.8 & 22.55 & 31.67 \\
\hline 28 & 61.7 & 36.8 & 19.05 & 31.67 \\
\hline 18 & 58.7 & 36.87 & 17.02 & 33.63 \\
\hline 8 & 57.3 & 37 & 17.01 & 38.00 \\
\hline 3 & 57.2 & 37.2 & 19.08 & 47.50 \\
\hline 2 & 55.7 & 37.25 & 18.11 & 50.67 \\
\hline
\end{tabular}

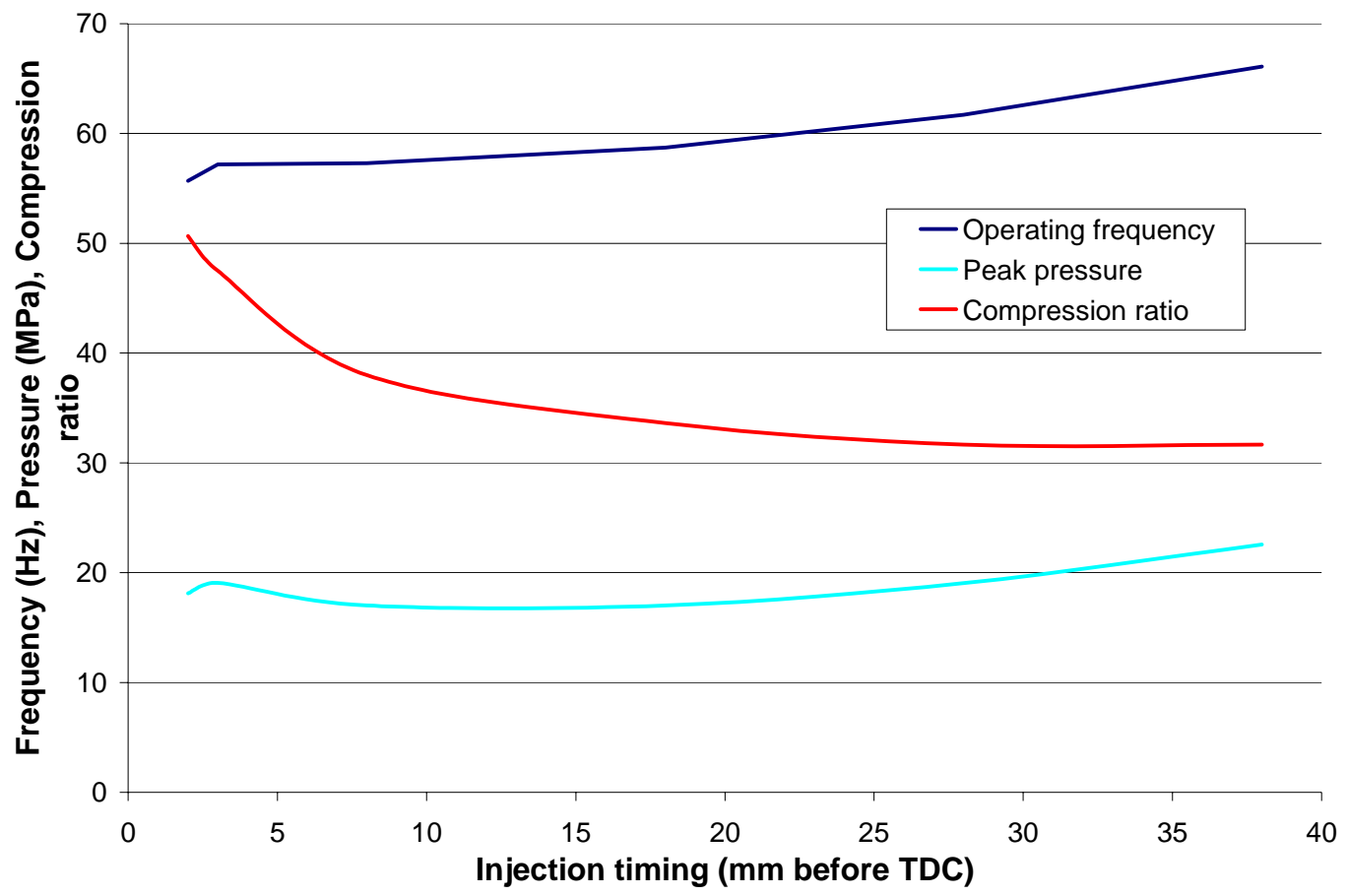

Figure 84. Operating frequency, peak pressure, and compression ratio vs. injection timing.

Advanced injection timing resulted in increased frequency and decreased compression ratio. Peak cylinder pressure was high for late and early injection with a decreased value in between. This was believed to be due to the following: as timing was advanced until about $10 \mathrm{~mm}$ before TDC, the heat release rate did not change much but the cylinder temperature was elevated for a longer period of time resulting in higher heat loss and lowered pressure than those in the case of late injection. Advancing the timing further, resulted in a larger portion of the heat release in premix burn that yielded a more sudden rise in pressure that yielded increased efficiency because of the HCCI operation. 
This phenomenon suggests that advancing injection timing marginally will not have the desired pseudo-HCCI effect on engine operation, it can even result in an efficiency drop. This suggests that fuel volatility is an important feature in terms of linear engines. It also suggests that the injector should be placed in the intake or scavenging duct to ensure thorough mixing and evaporation for HCCI operation.

\subsection{Effect of load}

The effect of load on operating frequency is shown in Figure 85. The amount of fuel injected was kept constant at $40 \mu \mathrm{l} /$ injection at $10 \mathrm{~mm}$ before TDC. Load was gradually increased until the engine stalled. Increasing the load caused the frequency to decrease. This effect was not unexpected as increasing load and decreasing fueling reduces the speed in conventional engines as well. However, the frequency of the linear engine as the function of load and fueling rate did not change as dramatically as it would in slider crank engines. This was mainly due to the fact that lowering the load and/or increasing fueling rate resulted higher operating frequency as well as higher compression ratio. The higher compression ratio yielded higher temperature and increased heat loss to the cylinder walls. Trends were similar for both simulation and real engine operation. The fact that increasing load reduces and increasing fueling rate increases the operating frequency makes operating frequency the obvious choice for a feedback parameter to control fuel quantity injected into the cylinder. 


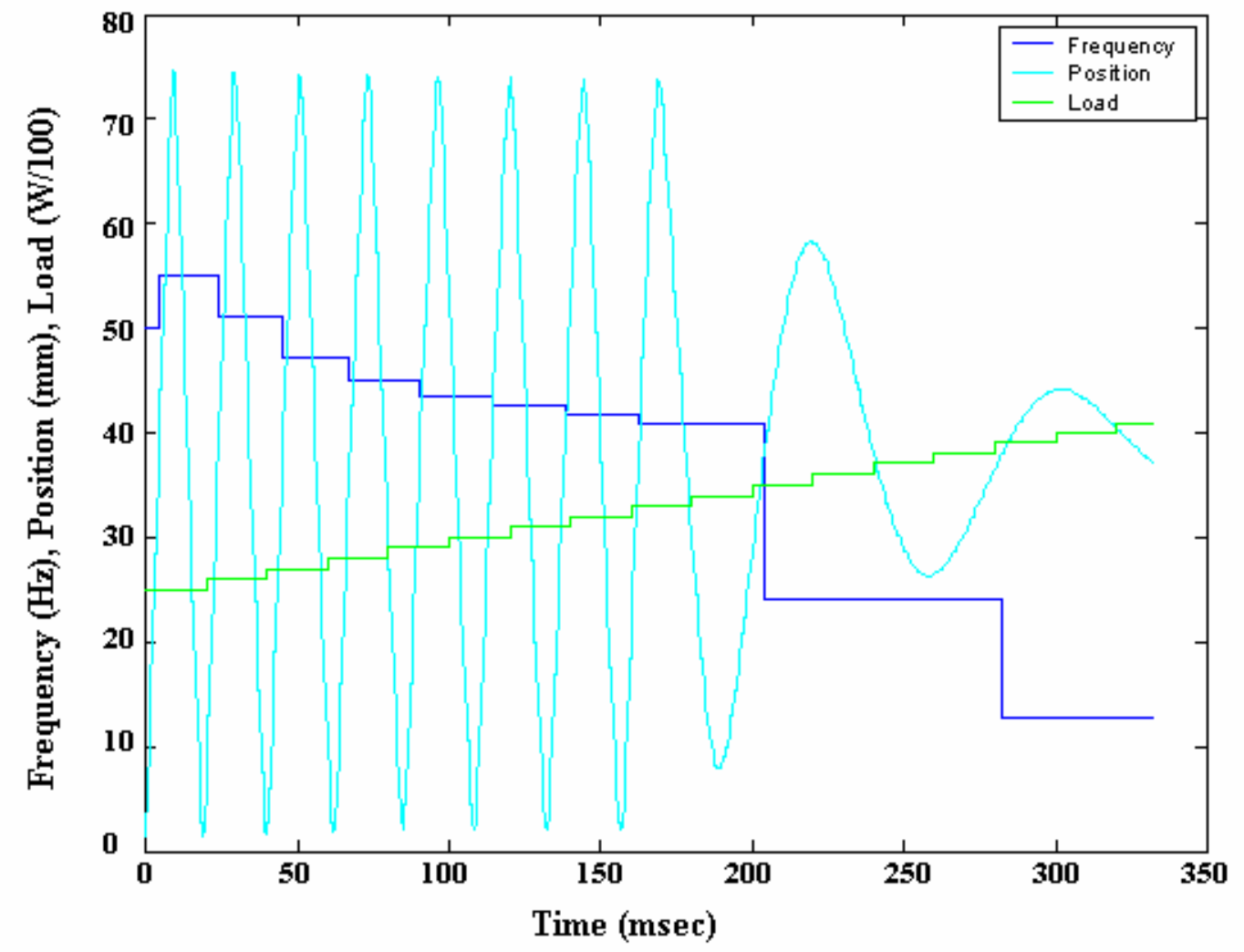

Figure 85. The effect of load on operating frequency.

\subsection{Results of the predictor/simulator model}

The predictor/simulator was created to find the set of engine parameters that yielded the highest mechanical efficiency. The predictor/simulator used a genetic algorithm and a neural network along with the simulation model in a recursive process. Section 5.2 described the model in detail and the results are described here.

Figure 86 shows the predicted efficiency and simulated efficiency for the iterative predicting/simulating process. The first fifty points were the initial randomly selected sets of engine parameters. It can be seen from the figure that predicted efficiency was always around $45-50 \%$ while the simulated efficiency varied between $10-45 \%$. The 
neural network had enough training data after 150 iterations (Simulation number 200) and the iterative process converged to the best efficiency point. The engine parameters that yielded the highest efficiency were: bore: $108.57 \mathrm{~mm}$, effective stroke: $27.14 \mathrm{~mm}$, free travel: -13.57 mm, translator mass: $1.96 \mathrm{~kg}$, amount of fuel injected: $8.9 \mu \mathrm{l} /$ injection, injection timing: $24.4 \mathrm{~mm}$ before TDC, ignition timing: $10.3 \mathrm{~mm}$ before TDC. This combination yielded: efficiency: $50.43 \%$, compression ratio: 13.12, operating frequency: $116 \mathrm{~Hz}$, Power output: $37.5 \mathrm{~kW}$. The full data set can be seen in Appendix B.

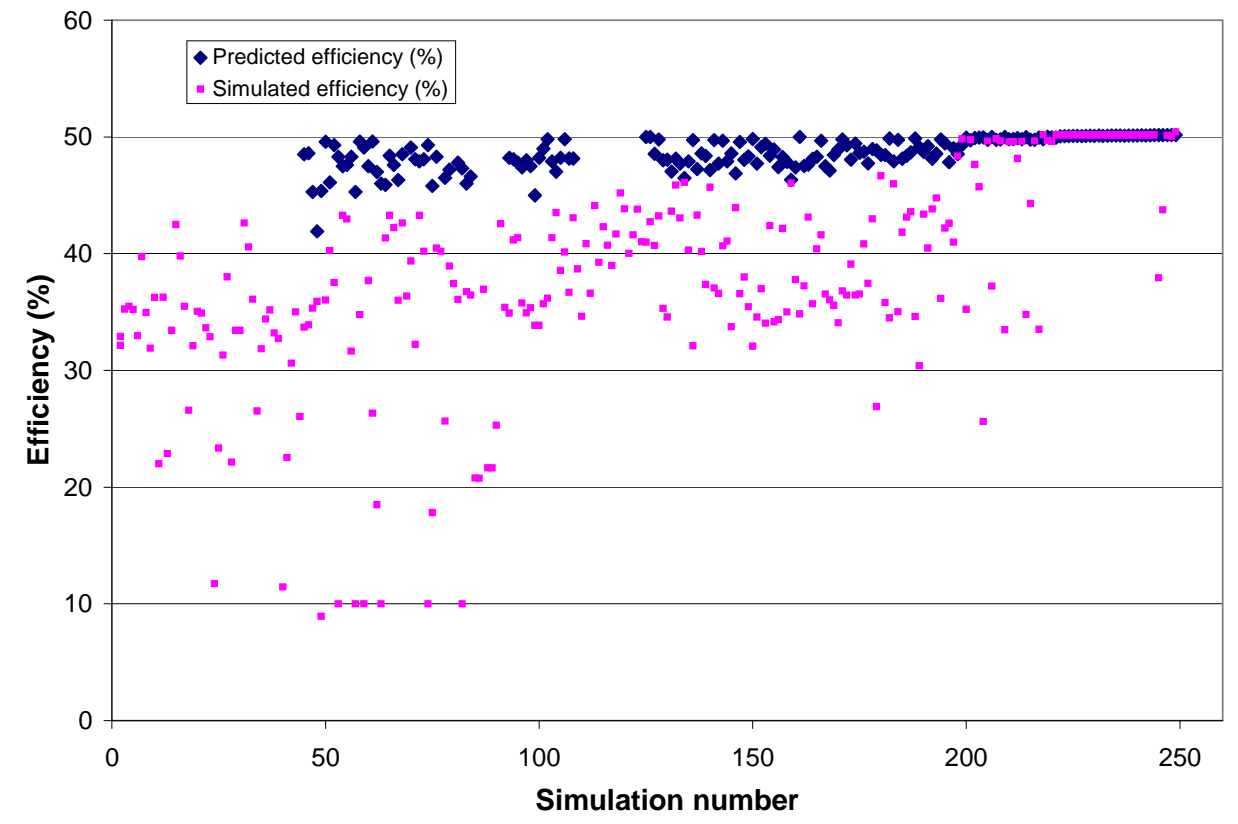

Figure 86. predicted and simulated engine efficiency.

The recursive nature of the predictor/simulator model gradually improved the accuracy of the neural network. It seemed that it jumped around at the beginning. But during that process, the ANN actually learned that which points are not the highest efficiency points. Once the ANN gathered enough data, it centered in and focused on the highest efficiency region. At the end of the iterative process the predictor/simulator 
changed and simulated variable sets in the vicinity of the best point, resulting in a fine resolution of the neural network around the highest efficiency point. This resulted in the ability of the neural network to predict with high accuracy around the highest efficiency point.

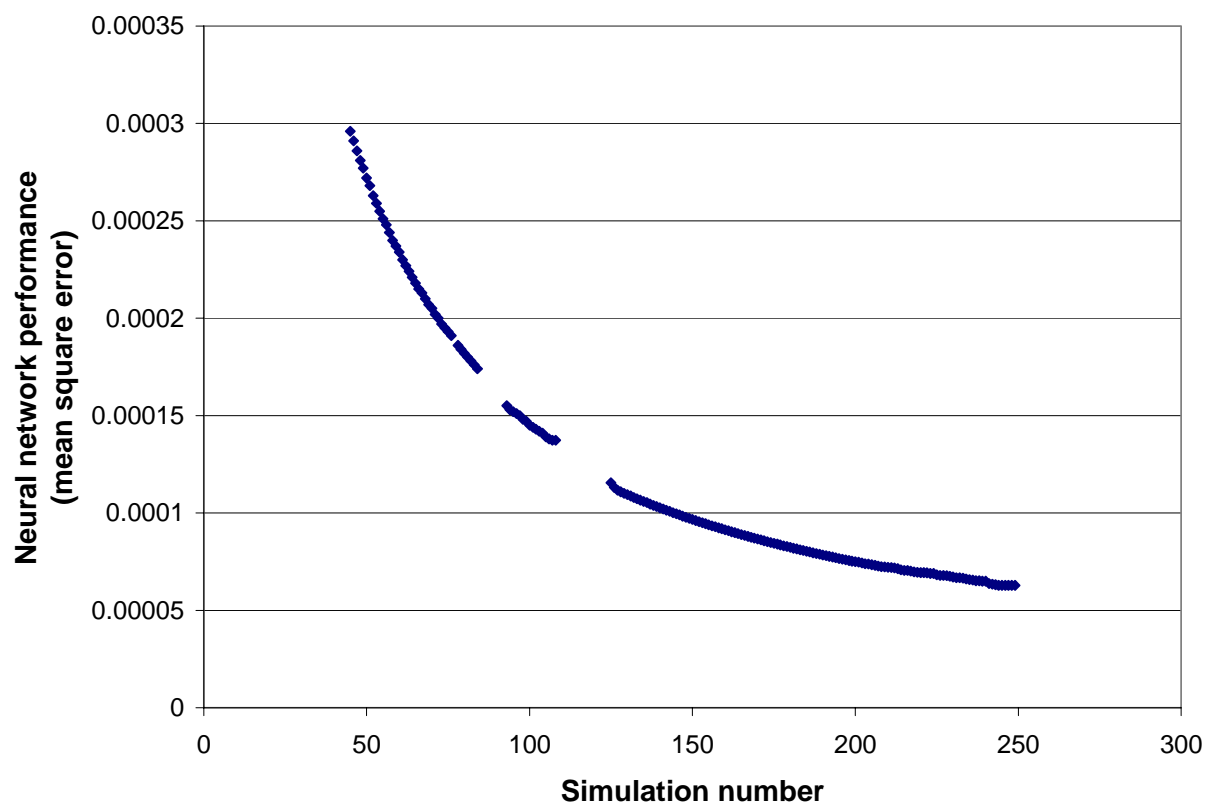

Figure 87. Performance of the artificial neural network. The graph shows the mean square error between the desired output and the output predicted by the ANN.

As more data points were provided to the neural network for training, the performance of the neural network gradually improved. The performance of an ANN is indicated by the mean square error. The mean square error is calculated by calculating the error between the desired output and the output the ANN predicted, squaring the error terms and finding the mean of the squared error set. Figure 87 shows the improving performance of the ANN as more data points were provided for training. The gaps in the graphs are missing data points, the result of unrecorded data. 


\subsection{Conclusions}

This chapter compared experimental results to simulation results. The effect of translator mass, stroke length, overcharging, fueling rate, injection timing, and load were compared. Increasing the translator mass resulted in decreased operating frequency and increased peak cylinder pressure and compression ratio. An engine with increased weight also showed a better ability to sustain operation and to sustain operation on less fuel. Increasing the stroke length resulted in decreased operating frequency and compression ratio, and increased peak pressures. Increased fueling rate and decreased load resulted in elevated peak pressure, compression ratio, and operating frequency. The extra fuel was wasted as heat loss to the cylinder walls and showed up as negative work on the pressure-position diagrams. The simulation results for injection timing showed similar trends to experimental operation. Advancing the injection timing resulted in decreasing peak cylinder pressure at the beginning. At more advanced timing, pressure increased again. This cautions against advancing the injection timing inadequately and reaching the lower efficiency region without reaching HCCI operation.

A set of engine parameters that resulted in the highest engine efficiency was found using a recursive predictor/simulator method. The results converged in about 150 iterations and yielded engine parameters. Table 12 shows the simulated engine parameters for the highest efficiency case and the parameters of the prototype engine for both cases with dimensions and dimensionless. The prototype engine parameters and the simulated highest efficiency engine parameters were significantly different and the data were not enough to draw any conclusions based on comparing engine parameters and results. 
Table 12. The combination of engine parameters that yielded the highest mechanical efficiency.

\begin{tabular}{|l|r|r|r|r|}
\hline & Simulated best efficiency engine & \multicolumn{2}{|c|}{ Prototype engine } \\
\cline { 2 - 5 } & With dimensions & Dimensionless & With dimensions & Dimensionless \\
\hline Bore (mm) & 108.5 & 1 & 76 & 1 \\
\hline Effective stroke (mm) & 27.140 & 0.25 & 38.000 & 0.5 \\
\hline Total stroke (mm) & 40.710 & 0.375 & 73.800 & 0.971 \\
\hline Fuel mass (g/injection) & 0.0074760 & 0.0038143 & 0.0031080 & 0.0011100 \\
\hline Load force (N) & 3970 & 3.368 & 138 & 0.131 \\
\hline Injection timing (mm before TDC) & 24.4 & 0.22 & 10 & 0.132 \\
\hline Operating frequency (Hz) & 116 & 0.049 & 53 & 0.024 \\
\hline Compression ratio & 13.12 & 13.12 & $\mathrm{na}$ & $\mathrm{na}$ \\
\hline Mechanical efficiency (\%) & 50.43 & $\mathrm{na}$ & 11 & $\mathrm{na}$ \\
\hline Power output (W) & 37,500 & $\mathrm{na}$ & 1080 & $\mathrm{na}$ \\
\hline
\end{tabular}

This simulation was performed for the engine only of the linear engine/alternator unit. The load was a friction brake applied on the connecting rod. This method allowed for any load at any frequency for any combination of the engine parameters. However, it is necessary to note that it would not be feasible to build a linear alternator with the mass of $1.96 \mathrm{~kg}$ that can move at $116 \mathrm{~Hz}$ and provide $37.5 \mathrm{~kW}$. The moving mass would be much larger than $1.96 \mathrm{~kg}$ for an engine that would provide $37.5 \mathrm{~kW}$. The common simulation of the engine and the alternator would be necessary for a proper optimization. 


\section{Conclusions}

After establishing the need for alternative fuel, engine, and vehicle research, hybrid configurations were described. It was shown that series hybrids, incorporating an internal combustion engine, have a future as the predecessor of fuel cell vehicles. It was also shown that linear engines would be strong candidates for auxiliary power unit application in series hybrid vehicles. The state of linear engine research was presented in detail. It was also concluded that the architecture of a linear engine would be multiple units of two-stroke, four-cylinder, combined dual-opposed piston arrangement units. This would eliminate the need for energy input (other than energy from fuel), it would minimize vibration, and allow for cylinder deactivation.

An analysis of the engine was carried out. Based on the analysis, a simulation model was constructed. The second-generation linear engine development at WVU was described and the prototype linear engine was presented. Experimental study and computer simulation were performed on the prototype and results were presented. Although the engine had run repeatedly, extensive experimentation was not feasible because of the high failure rate of components. Only a limited number of experiments was performed. The study included as independent variables bore, translator mass, stroke length, injection timing, load, amount of fuel injected, with dependent variables operating frequency, efficiency, and peak cylinder pressure as the base of comparison. The simulation data and the experimental data showed similar trends. Increasing the translator mass resulted in decreased operating frequency and increased peak cylinder pressure and compression ratio. An engine with increased translator mass also showed a better ability to sustain operation and to sustain operation on less fuel due to the increased 
compression ratio. Increasing the stroke length resulted in decreased operating frequency and compression ratio, and increased peak pressures. Increased fueling rate and decreased load resulted in elevated peak pressure, compression ratio, and operating frequency. The extra fuel was wasted as heat loss to the cylinder walls. This heat loss is a major energy loss in high compression ratio linear engines. Advancing the injection timing resulted in decreasing peak cylinder pressure at the beginning then at more advanced timing, pressure increased again. This cautions against advancing the injection timing inadequately and reach the lower efficiency region without reaching HCCI operation. To reach HCCI operation fuel should be injected into the intake or the scavenging ducts to let fuel mix and evaporate properly. It can also be concluded that frequency and compression ratio increase when fueling rate increases and/or when load decreases. This projects that frequency is going to be a prime candidate for input in the feedback control of future linear engines.

A set of engine parameters that resulted in the highest engine efficiency was found using a recursive predictor/simulator. The results converged in about 150 iterations yielding engine parameters and can be seen in Table 12 along with the prototype engine parameters.

Based on the results shown in this dissertation, it can be concluded that the highest efficiency for a linear engine would be reached using the recommended parameters above in a two-stroke, two-cylinder, opposed piston arrangement with HCCI operation. The HCCI operation reportedly results in decreased exhausted emissions. The vibration problem of the engine could be solved if the chosen architecture was combined dual-opposed piston arrangement. The design would make a powerful but efficient 
power source for a series hybrid SUV or a similar sized vehicle. Using three units of linear engines, arranged in the combined dual-opposed piston configuration, would result in a maximum power output of $225 \mathrm{~kW}$. The configuration would allow for cylinder deactivation to result in maximum efficiency that would yield better fuel consumption and lowered $\mathrm{CO}_{2}$ emissions. Decreasing the fuel consumption and exhaust emissions of the vehicles used in the urban areas would have a positive impact on the environment. 


\section{Recommendations for future work}

The next step of the present study should be to insert the linear alternator in the place of the present cranking equipment. Study should investigate the load profile and alternator behavior. Further tuning of the engine will be necessary to obtain efficiency data for the combined linear alternator/engine unit.

Future experimenting should investigate the effects of injecting fuel into the intake passages to achieve homogeneously charged compression ignition operation.

A closed loop controller of the linear engine should be designed to control fuel injection according to the load applied to the linear engine.

After optimization, emissions measurements should be performed on the linear engine.

A dual-opposed piston configuration linear engine should be designed and tested.

The linear engine should be inserted in a series hybrid electric vehicle and tested.

For extensive further testing redesign of the engine would be recommended. For the redesign of the engine some points that were learned during the course of this research are listed here to prevent others from doing the same mistakes.

\subsection{Lessons learned (positive)}

1. The sparking starting aid is a must for linear engines. Although it is only needed for the initial starting it is essential.

2. Micro-controllers provide a superior flexibility and simplicity over analogue controllers. They are highly recommended. 
3. Using off-the-shelf components is cost and time effective. However, it must be clear that "just the right component" does not exist. Modifications must be designed wisely.

4. Flexible injector lines must be used to withstand vibration.

5. Every electrical circuit must be fused. This can save a significant amount of money.

6. The connecting rod should be as short as possible with a constant diameter profile to remove stress concentrations. The danger of buckling was amplified by the fact that the engine had non-axial vibration.

7. A non-contact position sensor must be used. Using two of them at different locations would provide an insight into rod behavior.

8. Every electrical circuit should have a common ground. This includes the electric grid, power supplies, signal generators, data acquisition, control box, engine body, ignition system, linear alternator, cranking circuits, batteries, and the position sensor. This eliminated noise and voltage spikes in the system.

\subsection{Lessons learned (negative)}

1. Off-the-shelf components have their limitations. The urge to use twostroke cylinders off-the-shelf, resulted in a design where the cylinders were bolted on a base through angle iron. The angle iron flexed causing undesired radial vibration of the cylinders. This resulted in the frequent mechanical failure of the injectors, fittings, and cylinder bolts. The whole 
engine must be enclosed in a housing that does not allow radial vibration. Cylinder sleeves pressed in a housing would be an appealing idea.

2. No electrical circuit should operate on higher that $50 \mathrm{~V}$ for experimentation with the linear engine/alternator to prevent electric shock.

3. The engine has to have its own air supply.

4. The moving part of the position sensor must be built on a sturdy mount so it does not flex and introduce measuring error.

5. Better pressure sensors must be applied for the experiments. They should not drift with temperature.

6. Fuel should be injected in the intake instead of the cylinder to result in HCCI operation. This would eliminate the need for the high-pressure fuel system.

7. Data acquisition should start automatically whenever an attempt is made to start the engine and stop automatically when translator stops moving. This will help eliminate problems like unrecorded data when the engine sustains operation. 


\section{References}

1. http://hubbert.mines.edu Golden, CO, 2002

2. Matthews, R.; Lewis, D. J.; Davis, K.; “The Texas project, Part 5 - Economic analysis: CNG and LPG conversions of light-duty vehicle fleets” SAE-982447, Warrendale, PA, 1998

3. http://www.vision.net.au Sidney, Australia, 2002

4. http://lwf.ncdc.noaa.gov/oa/climate/gases.html\#wv Washington, DC, 2002

5. http://www.giss.nasa.gov/data/update/gistemp/graphs New York, NY, 2002

6. http://www.ngdc.noaa.gov/paleo/globalwarming/paleolast.html Boulder, CO, 2002

7. http://www.vehiclechoice.org/climate/greenhouse.html Sacramento, CA, 2002

8. http://unfccc.int/resource/convkp.html Bonn, Germany, 2002

9. http://www.dieselnet.com/links/health_html Brampton, Ontario, Canada, 2002

10. http://www.fhwa.dot.gov////environment/aqfactbk/factbk12.htm Washington, DC, 2002

11. “Automotive Fuel Economy Program Annual Update Calendar Year 2001” DOT HS 809 512, Washington, DC, 2001

12. http://www.britannica.com Chicago, IL, 2000

13. http://www.bergen.org/AAST/Projects/Engineering_Graphics/Drill/motor.html Hackensack, NJ, 2000

14. http://www.mtwp.k12.pa.us/schools/mtms/7white/524750.html Lancaster, PA, 2000

15. http://www.cybercomm.net/\%7Ejaguarca/students/B11/history.html New York, NY, 2000

16. http://www.hpo.hu/Magyar/ipsz/bendzsel.html\#33 Budapest, Hungary, 2000

17. Tóth-Nagy, C.; "Investigation and simulation of the planetary combination hybrid electric vehicle” Master's thesis at West Virginia University, Mechanical and Aerospace Engineering Department, Morgantown, WV, 2000

18. http://www.iea.org/pubs/studies/files/autofue/autofue.htm\#table Paris, France, 2000

19. http://www.ecomall.com/greenshopping/gm.htm Washington, DC, 2000

20. Schmidt, M. R.; Klemen, D.; "One-mode, input-split, parallel, hybrid transmission” United States Patent 5,558,595, Alexandria, VA, 1996

21. Schmidt, M. R.; “Two-mode, input-split, parallel, hybrid transmission” United States Patent 5,558,588, Alexandria, VA, 1996

22. Schmidt, M. R.; “Two-mode, compound-split, electro-mechanical vehicular transmission” United States Patent 5,558,589, Alexandria, VA, 1996

23. Schmidt, M. R.; “Two-mode, split power, electro-mechanical transmission” United States Patent 5,577,973, Alexandria, VA, 1996

24. http://www.fueleconomy.gov/feg/fcv_PEM.shtml Golden, CO, 2003

25. Briggs, A.; "Power of steam” ISBN 0718120760, University of Chicago Press, Chicago, IL, 1982 
26. Walker, G.; “Stirling engines” ISBN 0198562098, Clarendon Press, Oxford, United Kingdom, 1980

27. Hargreaves, C. M.; “The Phillips Stirling engine” ISBN 0444884637, Elsevier Science Publisher, New York, NY, 1991

28. Cummins, C. L. Jr.; “Internal fire” ISBN 0898837650, SAE Inc., Warrendale, PA, 1989

29. Hogland, L. C.; Percival, W. H.; Smith, J. L.; Syniuta, W.D.; Gerstmann, J.; “A technology evaluation of the Stirling engine for stationary power generation in the 500 to 2000 horsepower range” Report prepared for the US DOE, ORO/5392-01, Washington, DC, 1978

30. Kitzner, E. W.; “Automotive Stirling engine development program” Report prepared for NASA and US DOE, DOE/NASA/3496-4, NASA CR-159836, 1980

31. Shaltens, R. K.; Schreiber, J. G.; Wong, W. A.; "Update on the advanced Stirling conversion system project for $25 \mathrm{~kW}$ dish Stirling applications” SAE-929184, Warrendale, PA, 1992

32. Beale, W.; Holmes, W.; Lewis, S.; Cheng, E.; “Free piston Stirling enginesprogress report” SAE-730647, Warrendale, PA, 1973

33. Corey, J. A.; Yarr, G. A.; "Hots to Watts: The FPSE linear alternator system reinvented" SAE-929261, Warrendale, PA, 1992

34. Wood, J. G.; Lane, N, W.; Beale, W. T.; "Preliminary design of a 7 kW freepiston Stirling engine with rotary generator output” Proceedings of the $10^{\text {th }}$ International Stirling Engine Conference, Osnabruck, Germany, 2001

35. Kankam, M. D.; Rauch, J. S.; Santiago, W.; "Dynamic analysis of free-piston Stirling engine/linear alternator - load system - experimentally validated” SAE929265, Warrendale, PA, 1992

36. Dochat, G. D.; "Development of a small, free-piston Stirling engine, linearalternator system for solar thermal electric power applications” SAE-810457, Warrendale, PA, 1981

37. Hughes, W. O.; Goodnight, T. W.; McNelis, A. M.; “Overview of vibration testing of Stirling convertors" Presentation at the 2001 Spacecraft and Launch Vehicle Dynamic Environments Workshop, Washington, DC, 2001

38. Xu, Z. X.; Nasar, S. A.; Rosswurm, M.; "Stability analysis of free piston Stirling engine power generation system” SAE-929025, Warrendale, PA, 1992

39. Braun, A. T.; Schweitzer, P. H.; “The Braun linear engine” SAE-730185, Warrendale, PA, 1973

40. Somhorst, J. H. E.; Achten, P. A. J.; “The combustion process in a DI diesel hydraulic free piston engine” SAE-960032, Warrendale, PA, 1996

41. Prados, M. A.; “Towards a linear engine” Master's Thesis at Stanford University, Department of Mechanical Engineering, Stanford, CA, 2002

42. http://www.uqm.com/Technologies/images/Hmmwvwc.gif Frederick, CO, 2002

43. http://www.pei-idt.com/hmmwv1.htm Huntsville, AL, 2002

44. http://www.gm.com/company/gmability/environment/gm_and_the_env/releases/p aradigm_game_changing_010401.html Detroit, MI, 2002

45. http://www.evworld.com/archives/reports/inside_insight.html Papillion, NE, 2002

46. http://www.toyota.com Torrance, CA, 2002

47. http://www.hybridford.com/index.asp Detroit, MI, 2003 
48. http://www.gm.com/company/gmability/environment/gm_and_the_env/releases/p aradigm_hev_010401.html Detroit, MI, 2003

49. http://paslode.comproducts/tool_catalog/IM250A.html\#ops Vernon Hills, IL, 2004

50. Georgantas, A. I.; Krepec, T.; Cheng, R. M. H.; “Application of a double actuator electronic fuel control unit on a small gas turbine engine” SAE-912160, Warrendale, PA, 1991

51. Johansen, T. A.; Egeland, O.; Aa, E.; Kvamsdal, J.; Kvamsdal, R.; "Free-piston diesel engine timing and control towards electronic cam and crankshaft" Control Systems Technology, IEEE Transactions, Washington, DC, 2002

52. Johansen, T. A.; Egeland, O.; Aa, E.; Kvamsdal, J.; Kvamsdal, R.; "Free-piston diesel engine dynamics and control” Journal of Dynamic Systems, Measurement and Control, ASME, Libertyville, IL, 2003

53. Flinn, G. Jr.; “Observations of 25000 hours of free piston engine operation” SAE570042, Warrendale, PA, 1957

54. Noren, O. B.; Erwin, R. L.; "The future of the free-piston engine in commercial vehicles” SAE-580032, Warrendale, PA, 1958

55. Klotsch, P.; "Ford free-piston engine development” SAE-590045, Warrendale, PA, 1959

56. Zheng, Q.; “Characterization of the dynamic response of a cylinder deactivation valvetrain system” SAE-2001-01-0669, Warrendale, PA, 2001

57. Underwood, A. F.; "The GMR 4-4 "HYPEREX” engine. A concept of the freepiston engine for automotive use” SAE-570032, Warrendale, PA, 1957

58. Olson, D. R.; "Simulation of a free-piston engine with a digital computer" SAE580267, Warrendale, PA, 1958

59. Petreanu, S.; “Conceptual analysis of a four-stroke linear engine” Ph.D. dissertation at West Virginia University, Mechanical and Aerospace Engineering Department, Morgantown, WV, 2001

60. Achten, P. A. J.; Oever, J. P. J.; Vael, G. E. M.; "Design of a hydraulic free-piston engine” SAE Off-highway engineering conference, Warrendale, PA, 2001

61. Achten, P. A. J.; Oever, J. P. J.; Potma, J.; Vael, G. E. M.; "Horsepower with brains: The design of the CHIRON free-piston engine” SAE-2000-01-2545, Warrendale, PA, 2000

62. http://www.innas.com/Chiron_wp.html Breda, Netherlands, 2003

63. Achten, P. A. J.; “A review of free-piston engine concepts” SAE-941776, Warrendale, PA, 1994

64. Larmi, M.; Isaksson, S.; Tikkanen, S.; Lammila, M.; "Performance simulation of a compression ignition free piston engine” SAE-2001-01-0280, Warrendale, PA, 2001

65. Tikkanen, S.; Lammila, M.; "First cycles of the dual hydraulic free piston engine” SAE-2000-012546, Warrendale, PA, 2000

66. Beachley, N. H.; Fronczak, F. J.; "Design of a free-piston engine-pump” SAE921740, Warrendale, PA, 1992

67. Heintz, R. P.; "A free piston engine-pump for an automotive propulsion system” SAE-810266, Warrendale, PA, 1981 
68. Baruah, P. C.; “A free-piston engine hydraulic pump for an automotive propulsion system” SAE-880658, Warrendale, PA, 1988

69. Kaario, O.; "Comparison between single-step and two-step chemistry in a compression ignition free piston engine” SAE-2000-01-2937, Warrendale, PA, 2000

70. Goetz, M.; Peng, L.; “Free piston engine and its optimization” SAE-2000-010996, Warrendale, PA, 2000

71. Van Blarigan, P.; "Free-piston engine” United States Patent, US 6,199,519 B1, Alexandria, VA, 2001

72. Van Blarigan, P.; “Advanced internal combustion engine research” Proceedings of the 2000 DOE Hydrogen Program Review, Livermore, CA, 2000

73. http://www.eere.energy.gov/hydrogenandfuelcells/hydrogen/pdfs/28_snl_peter_v an_blarigan.pdf Golden, CO, 2004

74. Van Blarigan, P.; Paradiso, N.; Goldsborough, S.; "Homogeneous charge compression ignition with a free piston: a new approach to ideal Otto cycle performance” SAE-982484, Warrendale, PA, 1998

75. Pertl, A.; Personal communications, WVU, Morgantown, WV, 2002, 2003

76. Rerkpreedapong, D.; "Field analysis and design of a moving iron linear alternator for use with linear engine” Master's thesis at West Virginia University, Department of Computer Science and Electrical Engineering, Morgantown, WV, 1999

77. Bromborsky, A.; Cheskis, L.; Podlesak, T.; Famouri, P.; Clark, N. N.; Taylor, S.; “Analysis of a permanent magnet diesel alternator” ARO internal report 2002

78. Nandkumar, S.; “Two stroke linear engine” Master’s thesis at West Virginia University, Mechanical and Aerospace Engineering Department, Morgantown, WV, 1998

79. Cawthorne, W. R.; "Optimization of a brushless permanent magnet linear alternator for use with a linear internal combustion engine” Master's thesis at West Virginia University, Department of Computer Science and Electrical Engineering, Morgantown, WV, 1999

80. Houdyshell, D.; “Diesel two-stroke linear engine” Master's thesis at West Virginia University, Mechanical and Aerospace Engineering Department, Morgantown, WV, 2000

81. Atkinson, C. M.; Petreanu, S.; Clark, N. N.; Atkinson, R. J.; McDaniel, T.I.; Nandkumar, S.; Famouri, P.; "Numerical simulation of a two-stroke linear engine” SAE-1999-01-0921, Warrendale, PA, 1999

82. Clark, N. N.; Nandkumar, S.; Atkinson, C. M.; Atkinson, R. J.; McDaniel, T.I.; Petreanu, S.; Famouri, P.; Cawthorn, W.; "Experimental examination of the operation of a small bore two stroke linear engine” Proceedings of the Spring Technical Conference of the ASME International Combustion Engine Division, Libertyville, IL, 1998

83. Clark, N. N.; Nandkumar, S.; Famouri, P.; "Fundamental analysis of a linear twocylinder internal combustion engine” SAE-982692, Warrendale, PA, 1998

84. Shoukry, E.; Taylor, S.; Clark, N.; Famouri, P.; "Numerical simulation for parametric study of a two-stroke direct injection linear engine” SAE-2002-011739, Warrendale, PA, 2002 
85. Shoukry, E. F.; "Numerical simulation for parametric study of a two-stroke compression ignition direct injection linear engine” Ph.D. dissertation at West Virginia University, Mechanical and Aerospace Engineering Department, Morgantown, WV, 2003

86. Thompson, G. J.; Atkinson, C. M.; Clark, N. N.; Long, T. W.; and Hanzevack, E.L.; "Neural Network Modeling of the Emissions and Performance of a HeavyDuty Diesel Engine” Proceedings of Institution of Mechanical Engineers, Westminster, UK, 2000

87. Arsie, I.; Marotta, F.; Pianese, C.; and Rizzo, G.; "Information Based Selection of Neural Networks Training Data for S.I. Engine Mapping” SAE-2001-01-0561, Warrendale, PA, 2001

88. Ayeb, M.; Lichtenthäler, D.; Winsel, T.; and Theueerkauf, H.J.; "SI Engine Modeling Using Neural Networks” SAE-980790, Warrendale, PA, 1998

89. Tehranian, A.; "Effects of artificial neural neworks characterization on prediction of diesel engine emissions” Master's thesis at West Virginia University, Mechanical and Aerospace Engineering Department, Morgantown, WV, 2003

90. http://www.mathworks.com Natick, MA, 2004

91. Heywood, J. B.; “Internal combustion engine fundamentals” ISBN 0-07-028637X, McGraw-Hill, New York, NY, 1988

92. “Automotive handbook” Bosch, ISBN 3-18-418006-9, Robert Bosch GmbH, Munich, Germany, 1986

93. “Diesel accumulator fuel-injection system common rail” Bosch, Robert Bosch GmbH, Munich, Germany, 1999

94. Kim, S. S.; Kim, C. G.; Kim, C. U.; Pang, H. S.; Han, J. O.; Cho, Y. S.; “A study on efficiency and emission enhancements in a 4-stroke natural gas lean burn engine” SAE-960849, Warrendale, PA, 1996

95. Mendis, K. J. S.; Stone, C. R.; Ladommatos, N.; Weller, G.; “Modeling and measurements from a natural gas fuelled engine” SAE-930927, Warrendale, PA, 1993

96. Nakagawa, K.; Sakurai, T.; Shoji, F.; "Study of lean-burn gas engines using pilot fuel for ignition source” SAE-982480, Warrendale, PA, 1998

97. Christensen, M.; Johansson, B.; Einewall, P.; "Homogeneous charge compression ignition (HCCI) using isooctane, ethanol and natural gas-A comparison with spark-ignition operation” SAE-972874, Warrendale, PA, 1997

98. Gray, A. W.; Ryan, T. W.; "Homogeneous charge compression ignition (HCCI) of diesel fuel” SAE-971676, Warrendale, PA, 1997

99. Pucher, G. R.; Gardiner, D. P.; Bardon, M. F.; Battista, V.; “Alternative combustion systems for piston engines involving homogeneous charge compression ignition concepts-A review of studies using methanol, gasoline and diesel fuel” SAE-962063, Warrendale, PA, 1996

100. http://www.allisontransmission.com/product/electricdrive/ep50systembus.jsp Indianapolis, IN, 2002

101. http://www.lmcontrolsystems.com/PowerDrive.htm Rockville, MD, 2002

102. "Hybrid-electric drive heavy-duty vehicle testing project" Report, Submitted to Defense Advanced Research Projects Agency, Submitted by Northeast Advanced Vehicle Consortium, Agreement No.: NAVC1098-PG009837, Boston, MA, 2000 
103. Crosbie, G. M,; Murray, E.; Bauer, D. R.; Kim, H.; Park, S.; Vohs, J. M.; Gorte,R.J.; "Solid oxide fuel cells for direct oxidation of liquid hydrocarbon fuels in automotive auxiliary power units: Sulfur tolerance and operation on gasoline” SAE-2002-01-0410, Warrendale, PA, 2002

104. Zizelman, J.; Shaffer, S.; Mukerjee, S.; "Solid oxide fuel cell auxiliary power unit-A development update” SAE 2002-01-0411, Warrendale, PA, 2002

105. Gay-Desharnais, S. E.; Routex, J.; Holtzapple, M.; Ehsani,M.; "Investigation of hydrogen carriers for fuel-cell based transportation” SAE 2002-01-0097, Warrendale, PA, 2002

106. Ferguson, C. R.; “Internal combustion engines, Applied thermo sciences” ISBN 0-471-881295, Wiley \& Sons, New York, NY, 1985

107. Kellermeyer, W. F.; "Development and validation of a modular hybrid electric vehicle simulation model” Master’s thesis at West Virginia University, Mechanical and Aerospace Engineering Department, Morgantown, WV, 1998

108. Yen, J.; Langari, R.; “Fuzzy Logic” ISBN 0-13-525817-0, Prentice Hall, Upper Saddle River, NJ, 1999

109. Turak, E.; Personal communications, WVU, Morgantown, WV, 2002, 2003

110. Stanley, R.; Taraza, D.; Henein, N.; Bryzik, W.; “A simplified piston ring assembly friction model” Proceedings of the ASME Dynamic Systems and Control Division, Libertyville, IL, 1998

111. Stanley, R.; Taraza, D.; Henein, N.; Bryzik, W.; “A simplified friction model of the piston ring assembly" SAE-1999-01-0974, Warrendale, PA, 1999

112. Dearlove, J.; Cheng, W. K.; "Simultaneous piston ring friction and oil film thickness measurements in a reciprocating test rig” SAE-952470, Warrendale, PA, 1995

113. Marek, S. L.; Henein, N. A.; "Transient engine and piston friction during starting” SAE-922197, Warrendale, PA, 1992

114. Kuo, T.; Selinau, M. C.; Theobald, M. A.; Jones, J. D.; "Calculation of flow in the piston-cylinder-ring crevices of a homogeneous-charge engine and comparison with experiment” SAE-890838, Warrendale, PA, 1989

115. Ebner, H. W.; Jaschek, A. O.; "The importance of blow-by measurements, measuring equipment required and implementation” SAE-981081, Warrendale, PA, 1998

116. Tomanik, E.; Sobrinho, R. M. S.; Zecchinelli, R.; “Influence of top ring end gap types at blow-by of internal combustion engines” SAE-931669, Warrendale, PA, 1993

117. Young, D. F.; Munson, B. R.; Okiishi, T.H.; “A brief introduction to fluid mechanics” ISBN 0-471-36243-3, R. R. Wiley \& Sons, New York, NY, 2001

118. McDaniel, T.; Personal communications, NREL, Alton, CO, 2002, 2003

119. Muhammad, H.; Personal communications, WVU, Morgantown, WV, 2002, 2003

120. Young, M. B.; "Cyclic dispersion in the homogeneous-charge spark-ignition engine--A literature survey” Warrendale, PA, -810020, 1981

121. Glynn, T. E.; Personal communications, PCB Piezotronics, Depew, NY, 2003

122. Thompson, M.; Personal communications, MICRO-EPSILON, Raleigh NC, 2003, 2004 


\section{Appendix A}

Appendix A shows the testing results of the genetic algorithm. In the graphs, red indicates the high function values and blue indicates the low function values. It can be seen that the GA found the global maximums for both functions. The optimization process found the global maximum $100 \%$ of the time for the function

$z=3(1-x){ }^{2} e^{\left(-x^{2}-(y+1)^{2}\right)}-10\left(\frac{x}{5}-x^{3}-y^{5}\right) e^{\left(-x^{2}-y^{2}\right)}-\frac{1}{3} e^{\left(-(x+1)^{2}-y^{2}\right)}$

However, the optimization process focused in on local minimums in about $40 \%$ of the time for the function

$z=\sin 2 x-\sin 3 y+(x+y) 0.03$

nd was successful in about $60 \%$ of the time. 
Testing the genetic algorithm on the function:

$\mathrm{z}=3 *(1-\mathrm{x})^{2} * \exp \left(-\mathrm{x}^{2}-(\mathrm{y}+1)^{2}\right)-10 *\left(\mathrm{x} / 5-\mathrm{x}^{\wedge} 3-\mathrm{y} \wedge 5\right) * \exp \left(-\mathrm{x}^{2}-\mathrm{y}^{2}\right)-1 / 3 * \exp \left(-(\mathrm{x}+1)^{2}-\mathrm{y}^{2}\right)$
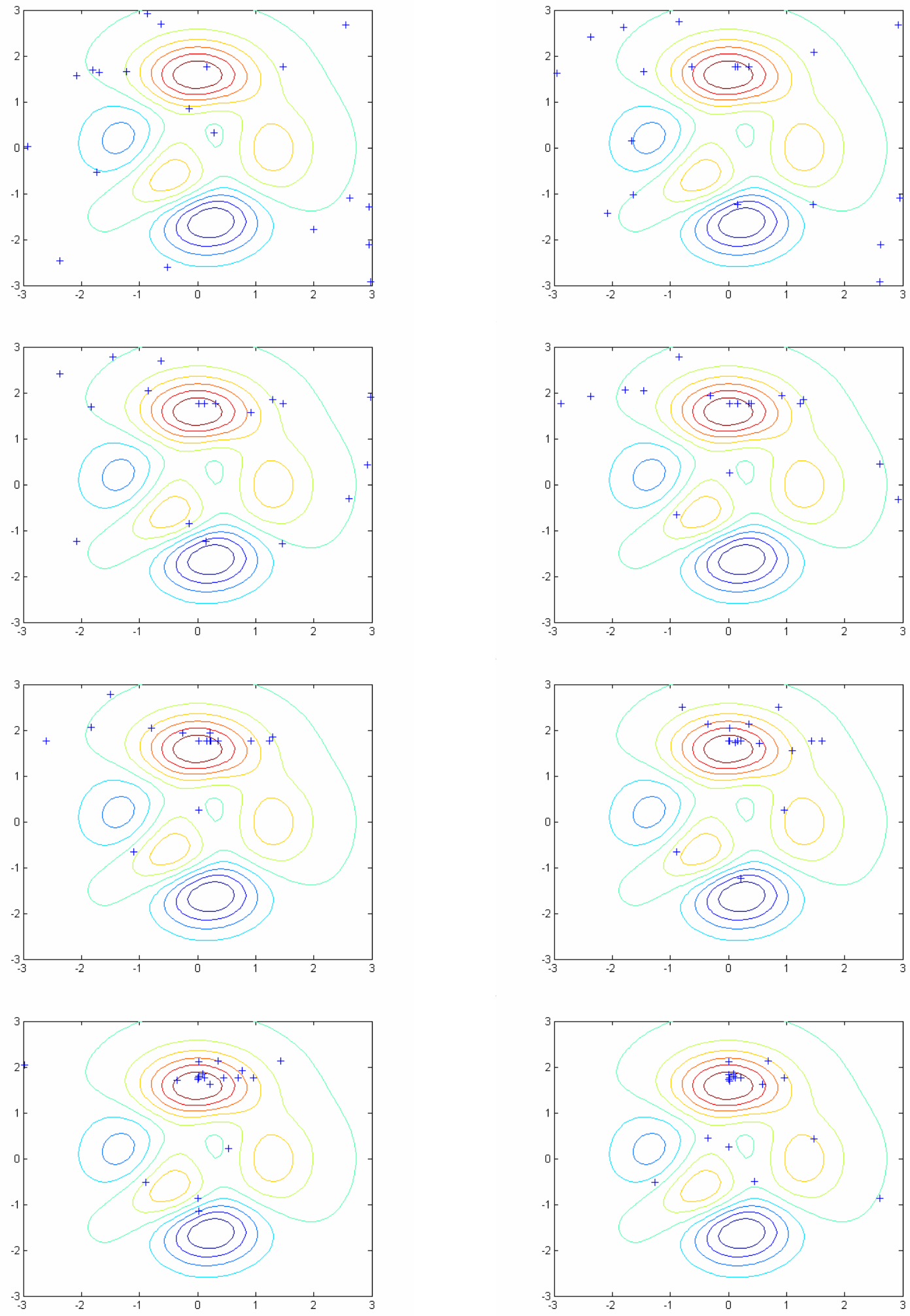

Genetic algorithm iterations 1 to 8 . 

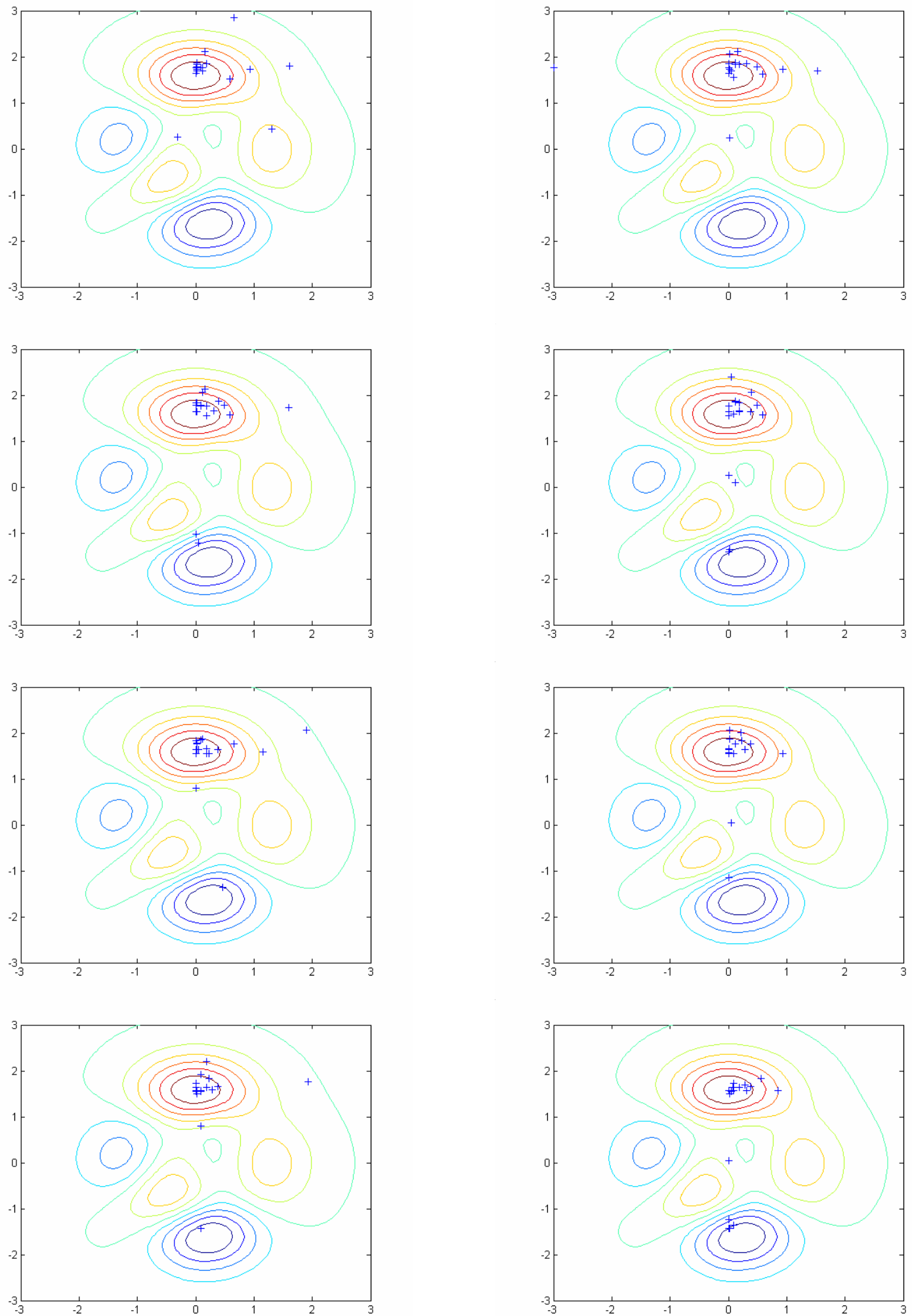

Genetic algorithm iterations 9 to 16 . 
Testing the genetic algorithm on the function: $z=\sin 2 x-\sin 3 y+(x+y) * 0.03$
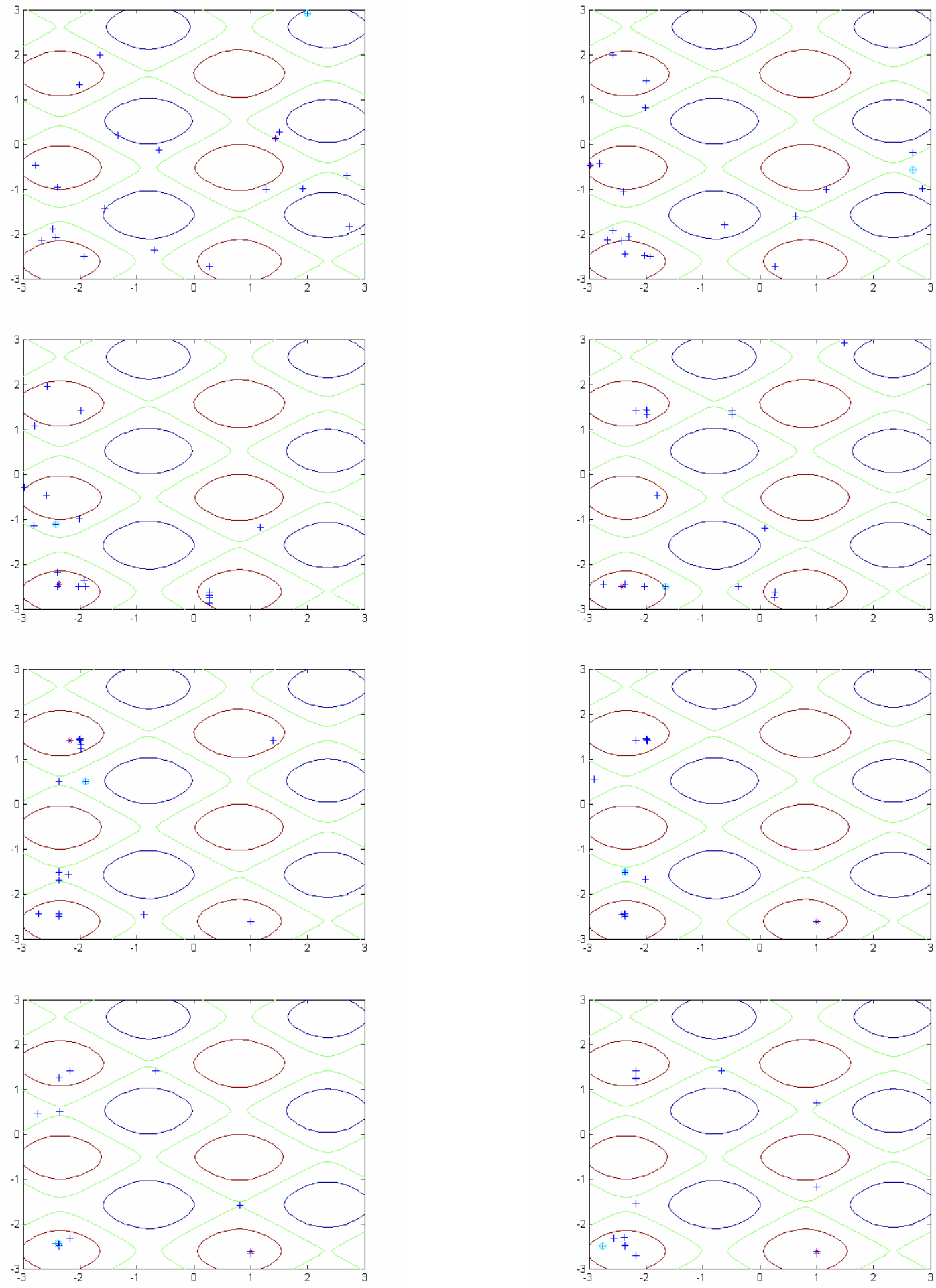

Genetic algorithm iterations 1 to 8. 

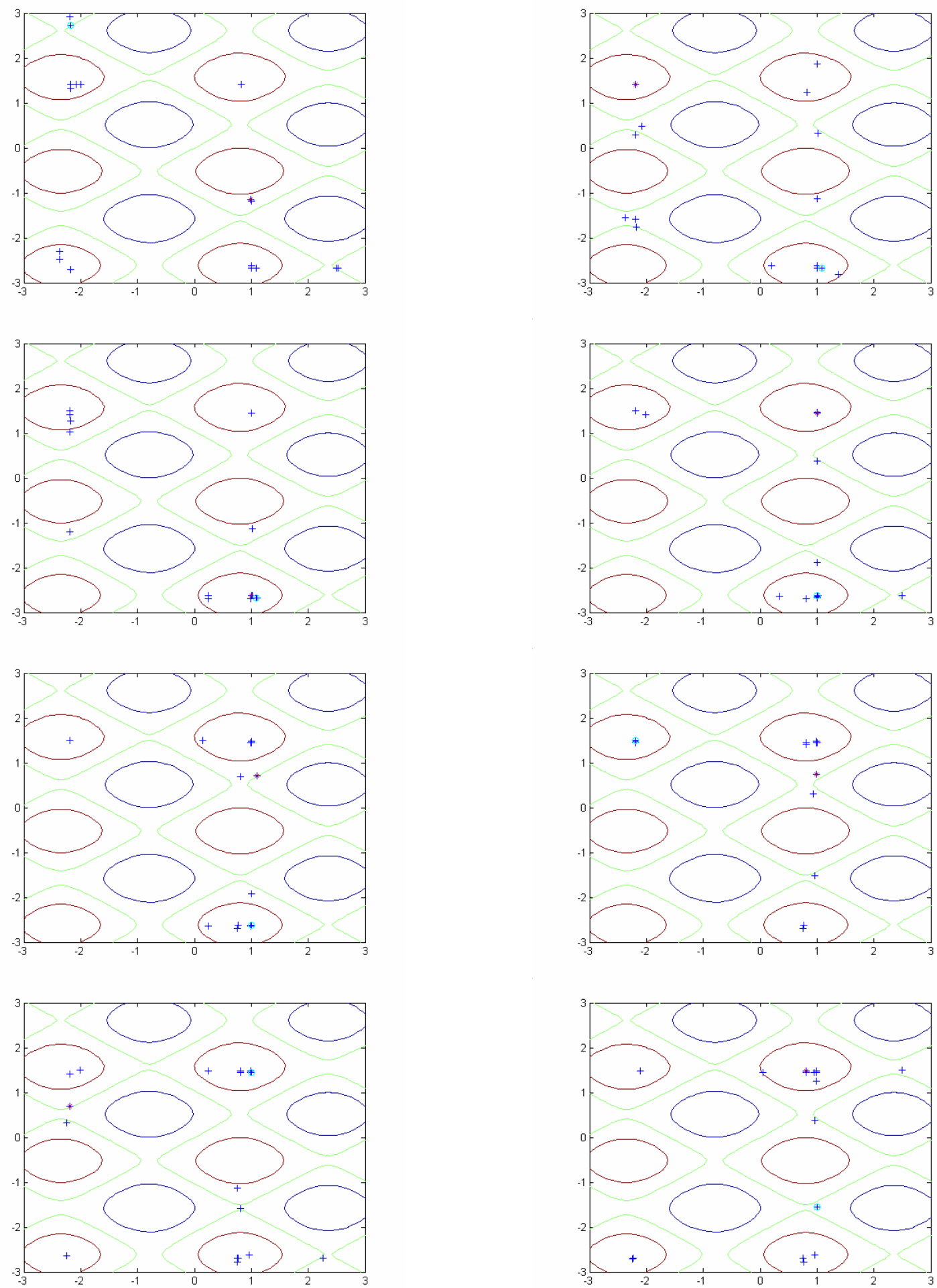

Genetic algorithm iterations 9 to 16 . 

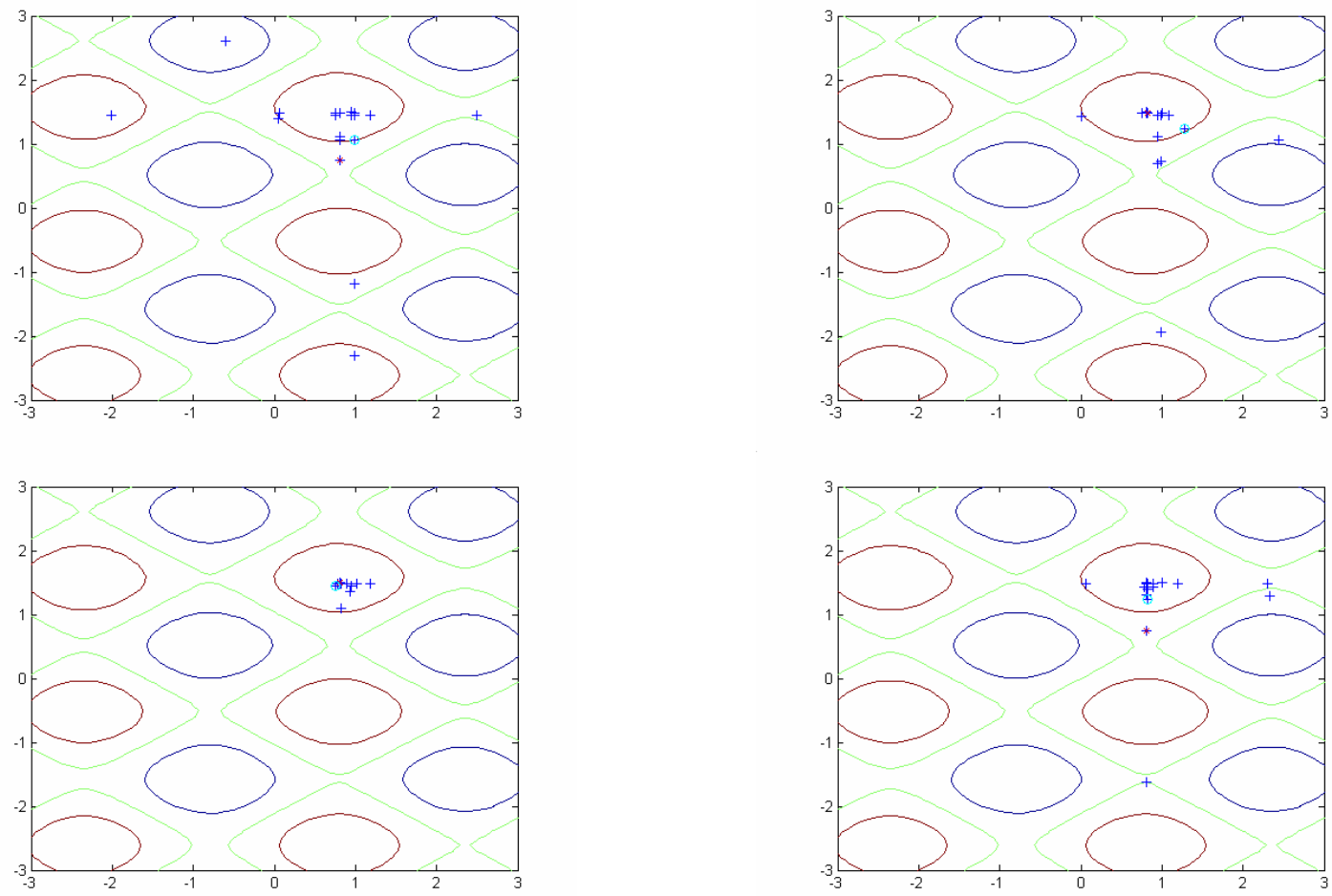

Genetic algorithm iterations 17 to 20 . 


\title{
Appendix B
}

\author{
Appendix B shows the sets of engine parameters that were found to result in the highest
}

efficiency for the given artificial neural network. Simulation results are also shown along with the engine variables.

\begin{tabular}{|c|c|c|c|c|c|c|c|c|c|c|c|c|c|c|}
\hline Run \# & $\begin{array}{l}\text { Bore } \\
(\mathrm{m})\end{array}$ & $\begin{array}{l}\text { Stroke eff. } \\
\text { (m) }\end{array}$ & $\begin{array}{l}\text { Free Travel } \\
\text { (m) }\end{array}$ & $\begin{array}{l}\text { Mass } \\
(\mathrm{kg})\end{array}$ & $\begin{array}{l}\text { Fuel injected } \\
(\mathrm{ml})\end{array}$ & $\begin{array}{l}\text { Injection timing } \\
\text { (mm before TDC) }\end{array}$ & $\begin{array}{l}\text { Ignition timing } \\
\text { (mm before TDC) }\end{array}$ & $\begin{array}{l}\text { Force on shaft } \\
\text { (N) }\end{array}$ & Compr. ratio & $\begin{array}{l}\text { Op. frequency } \\
(\mathrm{Hz})\end{array}$ & $\begin{array}{l}\text { Sim. efficiency } \\
(\%)\end{array}$ & $\begin{array}{l}\text { Power output } \\
\text { (W) }\end{array}$ & NN perform. & $\begin{array}{l}\text { Predicted eff. } \\
(\%)\end{array}$ \\
\hline 249 & 0.109 & 0.02714 & -0.01357 & 1.96020 & 0.0089 & $9 \quad 0.0244$ & 0.0103 & 416 & 13.1 & 117 & 50.4 & 4 (Iv) 37664 & $6.29 \mathrm{E}-05$ & 50.2 \\
\hline 248 & 0.109 & 0.02714 & -0.01357 & 1.96020 & 0.0089 & 0.0244 & 0.0103 & 413 & 13.3 & 116 & 50.1 & 36887 & 6.29E-05 & 50.2 \\
\hline 247 & 0.109 & 0.02714 & -0.01357 & 1.96024 & 0.0089 & 0.0244 & 0.0103 & 415 & 13.1 & 115 & 50.2 & 36651 & 6.29E-05 & 50.2 \\
\hline 246 & 0.121 & 0.03037 & -0.01519 & 2.74620 & 0.0125 & 0.0273 & 0.0115 & 588 & 7.4 & 93 & 43.7 & 42222 & $6.29 \mathrm{E}-05$ & 50.2 \\
\hline 245 & 0.109 & $\begin{array}{l}0.02714 \\
0.0271\end{array}$ & $\begin{array}{l}-0.0019 \\
-0.00003\end{array}$ & $\begin{array}{l}2.14020 \\
1.96024\end{array}$ & 0.0179 & 0.0244 & 0.0103 & 607 & $\begin{array}{l}7.4 \\
4.8\end{array}$ & $\begin{array}{l}93 \\
95\end{array}$ & $\begin{array}{l}4.1 \\
37.9\end{array}$ & 64273 & $\begin{array}{l}0.29 \mathrm{E}-05 \\
6.29 \mathrm{E}-05\end{array}$ & 50.2 \\
\hline 244 & 0.109 & 0.02714 & -0.01357 & 1.96024 & 0.0089 & 0.0244 & 0.0103 & 425 & 13.1 & 115 & 50.2 & 36309 & 6.29E-05 & 50.2 \\
\hline 243 & 0.109 & 0.02714 & -0.01357 & $\begin{array}{l}1.90024 \\
1.96024\end{array}$ & 0.0089 & 0.0244 & 0.0103 & 425 & $\begin{array}{l}13.1 \\
13.1\end{array}$ & 115 & 50.2 & 36309 & $6.31 \mathrm{E}-05$ & 50.1 \\
\hline 242 & 0.109 & 0.02714 & -0.01357 & 1.96024 & 0.0089 & 0.0244 & 0.0103 & 425 & 13.1 & 115 & 50.2 & 36309 & $6.34 \mathrm{E}-05$ & 50.1 \\
\hline 241 & 0.109 & 0.02714 & -0.01357 & $\begin{array}{l}1.90024 \\
1.96024\end{array}$ & 0.0089 & 0.0244 & 0.0103 & 425 & 13.1 & 115 & 50.2 & 36309 & 6.37E-05 & 50.1 \\
\hline 240 & 0.109 & 0.02714 & -0.01357 & 1.96024 & 0.0089 & 0.0244 & 0.0103 & 425 & 13.1 & 115 & 50.2 & 36309 & 6.49E-05 & 50.1 \\
\hline 239 & 0.109 & 0.02714 & -0.01357 & 1.96024 & 0.0089 & 0.0244 & 0.0103 & 425 & 13.1 & 115 & 50.2 & 36309 & 6.50E-05 & 50.1 \\
\hline 238 & 0.109 & 0.02714 & -0.01357 & 1.96024 & 0.0089 & 0.0244 & 0.0103 & 425 & 13.1 & 115 & 50.2 & 36309 & $6.52 \mathrm{E}-05$ & 50.1 \\
\hline 237 & 0.109 & 0.02714 & -0.01357 & 1.96024 & 0.0089 & 0.0244 & 0.0103 & 425 & 13.1 & 115 & 50.2 & 36309 & 6.52E-05 & 50.1 \\
\hline 236 & 0.109 & $\begin{array}{l}0.02714 \\
0.027\end{array}$ & -0.01357 & $\begin{array}{l}1.90024 \\
1.96024\end{array}$ & 0.0089 & $\begin{array}{l}0.0244 \\
0.0244\end{array}$ & 0.0103 & 425 & $\begin{array}{l}13.1 \\
13.1\end{array}$ & 115 & 50.2 & $\begin{array}{l}30009 \\
36309\end{array}$ & $\begin{array}{l}0.56 \mathrm{E}-05 \\
6.56 \mathrm{E}\end{array}$ & 50.1 \\
\hline 235 & 0.109 & 0.02714 & -0.01357 & 1.96024 & 0.0089 & 0.0244 & 0.0103 & 425 & 13.1 & 115 & 50.2 & 36309 & $6.57 \mathrm{E}-05$ & 50.1 \\
\hline 234 & 0.109 & 0.02714 & -0.01357 & $\begin{array}{l}1.90024 \\
\end{array}$ & 0.0089 & 0.0244 & 0.0103 & 425 & 13.1 & 115 & 50.2 & 36309 & $6.61 \mathrm{E}-05$ & 50.1 \\
\hline 233 & 0.109 & 0.02714 & -0.01357 & 1.96024 & 0.0089 & 0.0244 & 0.0103 & 425 & 13.1 & 115 & 50.2 & 36309 & 6.65E-05 & 50.1 \\
\hline 232 & 0.109 & 0.02714 & $\begin{array}{l}-0.01357 \\
-0.01357\end{array}$ & $\begin{array}{l}1.90024 \\
1.96024\end{array}$ & 0.0089 & $\begin{array}{l}0.0244 \\
0.024\end{array}$ & 0.0103 & 425 & $\begin{array}{l}13.1 \\
13.1\end{array}$ & 115 & $\begin{array}{l}50.2 \\
50.2\end{array}$ & $\begin{array}{l}30309 \\
36309\end{array}$ & $\begin{array}{l}0.05 E-05 \\
6.67 E-05\end{array}$ & 50.1 \\
\hline 231 & 0.109 & 0.02714 & -0.01357 & 1.96024 & 0.0089 & 0.0244 & 0.0103 & 425 & 13.1 & 115 & 50.2 & 36309 & 6.67E-05 & 50.1 \\
\hline 230 & 0.109 & 0.02714 & -0.01357 & 1.96024 & 0.0089 & 0.0244 & 0.0103 & 425 & 13.1 & 115 & 50.2 & 36309 & $6.71 \mathrm{E}-05$ & 50.1 \\
\hline 229 & 0.109 & 0.02714 & -0.01357 & 1.96024 & 0.0089 & 0.0244 & 0.0103 & 425 & 13.1 & 115 & 50.2 & 36309 & $6.74 \mathrm{E}-05$ & 50.1 \\
\hline 228 & 0.109 & 0.02714 & -0.01357 & 1.96024 & 0.0089 & 0.0244 & 0.0103 & 425 & 13.1 & 115 & 50.2 & 36309 & $6.78 \mathrm{E}-05$ & 50.1 \\
\hline 227 & 0.109 & 0.02714 & -0.01357 & 1.96024 & 0.0089 & 0.0244 & 0.0103 & 425 & 13.1 & 115 & 50.2 & 36309 & 6.78E-05 & 50.1 \\
\hline 226 & 0.109 & 0.02714 & -0.01357 & 1.96024 & 0.0089 & 0.0244 & 0.0103 & 425 & 13.1 & 115 & 50.2 & 36309 & 6.79E-05 & 50.1 \\
\hline 225 & 0.109 & 0.02714 & -0.01357 & $\begin{array}{l}1.90024 \\
\end{array}$ & 0.0089 & 0.0244 & 0.0103 & 425 & 13.1 & 115 & 50.2 & 36309 & $6.82 \mathrm{E}-05$ & 50.1 \\
\hline 224 & 0.109 & 0.02714 & -0.01357 & 1.96024 & 0.0089 & 0.0244 & 0.0103 & 425 & 13.1 & 115 & 50.2 & 36309 & 6.88E-05 & 50.1 \\
\hline 223 & 0.109 & 0.02714 & -0.01357 & $\begin{array}{l}1.90024 \\
\end{array}$ & 0.0089 & 0.0244 & 0.0103 & 425 & 13.1 & 115 & 50.2 & 36309 & $6.89 \mathrm{E}-05$ & 50.1 \\
\hline 222 & 0.109 & 0.02714 & -0.01357 & 1.96024 & 0.0089 & 0.0244 & 0.0103 & 425 & 13.1 & 115 & 50.2 & 36309 & 6.93E-05 & 50.1 \\
\hline 221 & 0.109 & 0.02714 & -0.01357 & 1.96024 & 0.0089 & 0.0244 & 0.0103 & 425 & 13.1 & 115 & 50.2 & 36309 & 6.93E-05 & 49.9 \\
\hline 220 & 0.105 & 0.02625 & -0.01313 & 1.77355 & 0.0079 & 0.0219 & 0.0085 & 384 & 12.9 & 116 & 49.6 & 32217 & 6.93E-05 & 50.0 \\
\hline 219 & 0.096 & 0.02389 & -0.01194 & 1.35165 & 0.0061 & 0.0215 & 0.0068 & 319 & 13.2 & 130 & 49.7 & 27570 & 6.95E-05 & 50.0 \\
\hline 218 & 0.097 & 0.02414 & -0.01207 & 1.38116 & 0.0063 & 0.0217 & 0.0078 & 336 & 12.5 & 126 & 50.2 & 28017 & 6.96E-05 & 49.8 \\
\hline 217 & 0.047 & 0.03536 & -0.00169 & 0.48320 & 0.0042 & 0.0318 & 0.0100 & 100 & 5.1 & 76 & 33.5 & 10988 & 7.01E-05 & 49.9 \\
\hline 216 & 0.093 & $\begin{array}{l}0.03530 \\
0.02336\end{array}$ & $\begin{array}{l}-0.01169 \\
-0.01168\end{array}$ & $\begin{array}{l}0.40320 \\
1.24922\end{array}$ & $\begin{array}{l}0.0055 \\
0.05\end{array}$ & 0.0210 & 0.0070 & 299 & $\begin{array}{r}r .1 \\
12.9\end{array}$ & $\begin{array}{r}130 \\
131\end{array}$ & $\begin{array}{l}3.5 \\
49.6\end{array}$ & 25352 & $\begin{array}{l}.04 \mathrm{E}-05 \\
7.04 \mathrm{E}\end{array}$ & $\begin{array}{l}49.9 \\
49.7\end{array}$ \\
\hline 215 & 0.096 & 0.02405 & -0.00601 & 1.37487 & 0.0093 & 0.0216 & 0.0097 & 423 & 7.6 & 114 & 44.3 & 39193 & 7.05E-05 & 49.8 \\
\hline 214 & 0.121 & 0.09097 & -0.02950 & 8.57945 & 0.0447 & 0.0817 & 0.0300 & 615 & 4.9 & 29 & 34.8 & 47598 & 7.08E-05 & $\begin{array}{l}49.0 \\
50.0\end{array}$ \\
\hline 213 & 0.093 & 0.02332 & -0.01166 & 1.23829 & 0.0056 & 0.0209 & 0.0042 & 304 & 13.0 & 133 & 49.6 & 26232 & 7.14E-05 & 49.8 \\
\hline 212 & 0.074 & 0.02242 & -0.01121 & 0.75710 & 0.0032 & 0.0201 & 0.0084 & 177 & 13.4 & 138 & 48.2 & 15194 & 7.17E-05 & 49.9 \\
\hline 211 & 0.093 & 0.02337 & -0.01168 & 1.24070 & 0.0055 & 0.0210 & 0.0048 & 298 & 13.1 & 132 & 49.6 & 25613 & 7.19E-05 & 49.8 \\
\hline 210 & 0.094 & 0.02341 & -0.01171 & 1.25817 & 0.0056 & 0.0210 & 0.0088 & 300 & 13.0 & 131 & 49.6 & 25467 & 7.21E-05 & 49.8 \\
\hline 209 & 0.059 & $\begin{array}{l}0.02341 \\
0.02018\end{array}$ & 0.00989 & $\begin{array}{l}1.25017 \\
0.54821\end{array}$ & $\begin{array}{l}0.0034 \\
0.036\end{array}$ & 0.0131 & 0.0098 & 121 & $\begin{array}{r}1.0 \\
4.9\end{array}$ & 86 & $\begin{array}{l}49.0 \\
33.5\end{array}$ & $\begin{array}{r}2401 \\
9110\end{array}$ & $\begin{array}{l}1.22 \mathrm{E}-05 \\
7.22 \mathrm{C}\end{array}$ & $\begin{array}{l}49.8 \\
50.0\end{array}$ \\
\hline 208 & 0.093 & 0.02344 & $\begin{array}{r}-0.01172 \\
\end{array}$ & 1.24758 & 0.0057 & 0.0211 & 0.0023 & 304 & $\begin{array}{l}13.0 \\
\end{array}$ & 132 & 49.7 & 26321 & 7.24E-05 & 49.8 \\
\hline 207 & 0.097 & 0.02414 & -0.01207 & $\begin{array}{l}1.24108 \\
1.37847\end{array}$ & 0.0063 & 0.0217 & 0.0025 & $\begin{array}{l}304 \\
336\end{array}$ & 12.7 & 127 & 49.8 & $\begin{array}{l}20321 \\
28591\end{array}$ & $\begin{array}{l}1.24 \mathrm{E}-05 \\
7.28 \mathrm{E}\end{array}$ & $\begin{array}{l}49.8 \\
49.7\end{array}$ \\
\hline 206 & 0.093 & 0.02334 & -0.00693 & 2.47009 & 0.0079 & 0.0023 & 0.0067 & 589 & 4.9 & 80 & 37.2 & 23844 & 7.31E-05 & 50.0 \\
\hline 205 & 0.093 & 0.02328 & -0.01159 & 1.23587 & 0.0057 & 0.0209 & 0.0030 & 304 & $\begin{array}{l}4.5 \\
13.0\end{array}$ & 133 & 49.6 & 26316 & 7.34E-05 & 49.7 \\
\hline 204 & 0.012 & 0.00895 & -0.00434 & 0.02324 & 0.0000 & 0.0042 & 0.0044 & 5 & 13.2 & 184 & 25.6 & 123 & 7.37E-05 & 50.0 \\
\hline 203 & 0.079 & 0.02395 & -0.01050 & $\begin{array}{l}0.91212 \\
0.91212\end{array}$ & 0.0047 & 0.0167 & $\begin{array}{l}0.0024 \\
0\end{array}$ & 226 & 11.5 & 125 & 45.7 & $\begin{array}{l}19706 \\
1970\end{array}$ & 7.39E-05 & 49.9 \\
\hline 202 & 0.121 & $\begin{array}{l}0.02395 \\
0.03034\end{array}$ & $\begin{array}{l}-0.01050 \\
-0.01517\end{array}$ & $\begin{array}{l}0.91212 \\
2.73866\end{array}$ & 0.0125 & 0.0273 & $\begin{array}{l}0.0034 \\
0.0031\end{array}$ & $\begin{array}{l}220 \\
545\end{array}$ & $\begin{array}{r}11.5 \\
9.7\end{array}$ & $\begin{array}{r}125 \\
99\end{array}$ & $\begin{array}{l}45.1 \\
47.6\end{array}$ & $\begin{array}{l}19068 \\
44087\end{array}$ & $\begin{array}{l}1.39 \mathrm{E}-05 \\
7.43 \mathrm{E}-05\end{array}$ & $\begin{array}{l}49.9 \\
49.9\end{array}$ \\
\hline 201 & 0.098 & 0.02447 & -0.01224 & 1.43648 & 0.0065 & 0.0220 & 0.0026 & 334 & $\begin{array}{r}1.1 \\
13.1\end{array}$ & 127 & 49.8 & 28910 & 7.47E-05 & 49.7 \\
\hline 200 & 0.121 & 0.08340 & $\begin{array}{l}-0.01224 \\
-0.02232\end{array}$ & $\begin{array}{l}1.43040 \\
9.47083\end{array}$ & 0.0413 & 0.0416 & 0.0417 & 668 & $\begin{array}{r}4.1 \\
4.7\end{array}$ & 27 & $\begin{array}{l}49.8 \\
35.2\end{array}$ & 41054 & $\begin{array}{l}1.49 \mathrm{E}-05 \\
7.49 \mathrm{E}\end{array}$ & $\begin{array}{l}49.1 \\
49.9\end{array}$ \\
\hline 199 & 0.093 & 0.02328 & -0.01164 & 1.24553 & 0.0056 & 0.0209 & 0.0023 & 304 & 13.1 & 135 & 49.8 & 27078 & $7.52 \mathrm{E}-05$ & 49.3 \\
\hline 198 & 0.071 & 0.01776 & -0.00888 & 0.69113 & 0.0025 & 0.0160 & 0.0018 & 195 & 12.9 & 152 & 48.4 & 12817 & 7.56E-05 & 48.5 \\
\hline 197 & 0.118 & 0.04194 & 0.02093 & 10.57265 & 0.0315 & 0.0356 & 0.0042 & 728 & 9.5 & 33 & 41.0 & 35032 & 7.59E-05 & 49.0 \\
\hline 196 & 0.094 & $\begin{array}{l}0.06996 \\
0.069\end{array}$ & 0.00868 & $\begin{array}{r}3.78298 \\
\end{array}$ & 0.0288 & 0.0070 & 0.0070 & 302 & 9.6 & 39 & 42.6 & $\begin{array}{l}40438 \\
\end{array}$ & 7.63E-05 & $\begin{array}{l}43.0 \\
47.8\end{array}$ \\
\hline 195 & 0.037 & 0.00937 & -0.00463 & 0.17971 & 0.0004 & 0.0075 & 0.0016 & 62 & 13.5 & 212 & 42.2 & 2229 & 7.66E-05 & 49.4 \\
\hline 194 & 0.090 & 0.02508 & -0.00005 & 2.78101 & 0.0112 & 0.0222 & 0.0125 & 608 & $\begin{array}{r}5.0 \\
\end{array}$ & 69 & 36.2 & 29327 & 7.00E-05 & $\begin{array}{l}49.4 \\
49.8\end{array}$ \\
\hline 193 & 0.121 & 0.03601 & 0.00517 & 3.48467 & 0.0297 & 0.0221 & 0.0036 & 597 & 9.9 & 77 & 44.8 & 83947 & 7.73E-05 & 48.6 \\
\hline 192 & 0.039 & 0.01491 & -0.00740 & 0.26856 & 0.0006 & 0.0134 & 0.0033 & 63 & $\begin{array}{r}12.9 \\
\end{array}$ & 144 & 43.8 & 2635 & 7.77E-05 & 48.1 \\
\hline $\begin{array}{l}192 \\
191\end{array}$ & 0.075 & $\begin{array}{l}0.05113 \\
0.0513\end{array}$ & 0.02541 & $\begin{array}{l}0.20830 \\
1.77146\end{array}$ & 0.0128 & $\begin{array}{l}0.0464 \\
0.0460\end{array}$ & 0.0051 & $\begin{array}{r}03 \\
143\end{array}$ & $\begin{array}{r}1.9 \\
9.6\end{array}$ & $\begin{array}{r}144 \\
46\end{array}$ & $\begin{array}{l}43.8 \\
40.5\end{array}$ & $\begin{array}{r}2035 \\
19924\end{array}$ & $\begin{array}{l}7.80 \mathrm{E}-05 \\
\text { S }\end{array}$ & $\begin{array}{l}40.1 \\
49.2\end{array}$ \\
\hline 190 & 0.094 & 0.02341 & $\begin{array}{r}-0.00002 \\
\end{array}$ & 1.88972 & 0.0115 & 0.0141 & 0.0023 & 466 & 9.0 & 100 & 43.4 & 41182 & 7.84E-05 & 48.7 \\
\hline $\begin{array}{l}190 \\
189\end{array}$ & 0.036 & $\begin{array}{l}0.02341 \\
0.01333\end{array}$ & $\begin{array}{r}0.00665 \\
0.0065\end{array}$ & $\begin{array}{l}1.808912 \\
0.30906\end{array}$ & 0.0008 & 0.0051 & 0.0031 & $\begin{array}{r}400 \\
59\end{array}$ & $\begin{array}{l}.0 .9 \\
4.9\end{array}$ & 80 & $\begin{array}{l}43.4 \\
30.4\end{array}$ & $\begin{array}{r}41182 \\
1670\end{array}$ & $\begin{array}{l}.84 \mathrm{E}-05 \\
7.88 \mathrm{E}\end{array}$ & $\begin{array}{l}4.1 \\
49.1\end{array}$ \\
\hline 188 & 0.085 & 0.02124 & 0.01052 & 0.93965 & 0.0080 & 0.0022 & 0.0097 & 244 & 4.7 & 93 & 34.6 & 23821 & 7.92E-05 & 49.9 \\
\hline $\begin{array}{l}100 \\
187\end{array}$ & 0.078 & 0.03777 & 0.00395 & 1.39307 & 0.0127 & 0.0339 & 0.0038 & 233 & 9.8 & 78 & $\begin{array}{l}43.6 \\
\text { a }\end{array}$ & 36008 & 7.95E-05 & 48.7 \\
\hline $\begin{array}{l}187 \\
186\end{array}$ & 0.090 & 0.02419 & $\begin{array}{r}0.03950 \\
-0.01210\end{array}$ & $\begin{array}{l}1.39301 \\
2.39055\end{array}$ & 0.0050 & $\begin{array}{l}0.0084 \\
0.039\end{array}$ & $\begin{array}{l}0.0028 \\
0.024\end{array}$ & 348 & $\begin{array}{l}9.8 \\
9.6\end{array}$ & 88 & $\begin{array}{l}43.6 \\
43.1\end{array}$ & $\begin{array}{l}3008 \\
14117\end{array}$ & $\begin{array}{l}1.95 \mathrm{E}-05 \\
7.99 \mathrm{E}-0\end{array}$ & $\begin{array}{l}48.1 \\
48.4\end{array}$ \\
\hline 185 & 0.121 & 0.03660 & -0.01830 & 8.47383 & 0.0122 & 0.0302 & 0.0037 & 600 & 9.9 & 54 & 41.8 & 21066 & 8.03E-05 & 48.1 \\
\hline 184 & 0.121 & 0.03437 & 0.01665 & 9.30109 & 0.0283 & 0.0288 & 0.0153 & 916 & 4.8 & 36 & 35.0 & 33967 & 8.07E-05 & 49.7 \\
\hline $\begin{array}{l}104 \\
183\end{array}$ & 0.121 & 0.03037 & $\begin{array}{l}-0.01507 \\
\end{array}$ & 2.99293 & 0.0126 & 0.0190 & 0.0030 & 560 & $\begin{array}{l}9.0 \\
9.7\end{array}$ & 95 & 46.0 & 41386 & $8.11 \mathrm{E}-05$ & $\begin{array}{l}47.9 \\
4.9\end{array}$ \\
\hline 182 & 0.052 & 0.03140 & 0.01236 & 0.87164 & 0.0040 & 0.0034 & 0.0056 & 107 & 5.5 & 51 & 34.5 & 6405 & 8.15E-05 & 49.9 \\
\hline 181 & 0.086 & 0.06027 & -0.00571 & 3.02735 & 0.0217 & 0.0060 & 0.0301 & 360 & 5.0 & 42 & 35.8 & 33013 & 8.19E-05 & 48.5 \\
\hline 180 & 0.119 & 0.02988 & -0.00581 & 2.60366 & 0.0190 & 0.0244 & 0.0030 & 606 & 9.4 & 101 & 46.7 & 72063 & 8.23E-05 & 48.5 \\
\hline 179 & 0.027 & 0.00812 & 0.00398 & 0.10938 & 0.0003 & 0.0030 & 0.0016 & 36 & 5.0 & 136 & 26.9 & 1088 & 8.27E-05 & 48.9 \\
\hline 178 & 0.120 & 0.02990 & $\begin{array}{c}-0.00006 \\
\end{array}$ & 2.61984 & 0.0191 & 0.0030 & 0.0030 & 512 & 9.8 & 92 & 43.0 & 62480 & $8.31 \mathrm{E}-05$ & 49.0 \\
\hline 177 & 0.105 & 0.07781 & 0.02014 & 14.39463 & 0.0462 & 0.0091 & 0.0097 & 641 & 7.2 & 19 & 37.5 & 28215 & 8.35E-05 & 47.7 \\
\hline
\end{tabular}




\begin{tabular}{|c|c|c|c|c|c|c|c|c|c|c|c|c|c|c|}
\hline Run \# & $\begin{array}{l}\text { Bore } \\
(\mathrm{m})\end{array}$ & $\begin{array}{l}\text { Stroke eff. F } \\
(\mathrm{m})\end{array}$ & $\begin{array}{l}\text { Free Travel } \\
(\mathrm{m})\end{array}$ & $\begin{array}{l}\text { Mass } \\
(\mathrm{kg})\end{array}$ & $\begin{array}{l}\text { Fuel injected } \\
(\mathrm{ml})\end{array}$ & $\begin{array}{l}\text { Injection timing } \\
\text { (mm before TDC) }\end{array}$ & $\begin{array}{l}\text { Ignition timing } \\
\text { (mm before TDC) }\end{array}$ & $\begin{array}{l}\text { Force on shaft } \\
\text { (N) }\end{array}$ & Compr. ratio & $\begin{array}{l}\text { Op. frequency } \\
(\mathrm{Hz})\end{array}$ & $\begin{array}{l}\text { Sim. efficiency } \\
(\%)\end{array}$ & $\begin{array}{l}\text { Power output } \\
\text { (W) }\end{array}$ & NN perform. & $\begin{array}{l}\text { Predicted eff. } \\
(\%)\end{array}$ \\
\hline 176 & 0.042 & 0.01235 & -0.00492 & 0.16622 & 0.0007 & 0.0092 & 0.0058 & 70 & 10.2 & 207 & 40.8 & 4678 & 8.40E-05 & 48.7 \\
\hline 175 & 50.121 & 0.03094 & 0.01055 & 5.66874 & 0.0207 & 0.0206 & 0.0106 & 731 & 4.8 & 46 & 36.6 & 32806 & $8.44 \mathrm{E}-05$ & 48.6 \\
\hline 174 & 0.090 & 0.02879 & -0.01375 & 1.96408 & 0.0055 & 0.0153 & 0.0111 & 361 & 4.7 & 75 & 36.5 & 15770 & 8.48E-05 & 49.4 \\
\hline 173 & 0.056 & 0.02211 & -0.00002 & 1.19305 & 0.0038 & 0.0088 & 0.0025 & 213 & 8.6 & 74 & 39.1 & 9227 & $8.53 \mathrm{E}-05$ & 48.1 \\
\hline 172 & 20.103 & 0.02851 & 0.00003 & 4.24136 & 0.0148 & 0.0256 & 0.0061 & 735 & 4.8 & 57 & 36.5 & 31027 & $8.57 \mathrm{E}-05$ & 49.3 \\
\hline 171 & 0.108 & 0.05258 & -0.01602 & 4.36254 & 0.0196 & 0.0094 & 0.0257 & 524 & 4.7 & 45 & 36.8 & 33081 & $8.61 \mathrm{E}-05$ & 49.8 \\
\hline 170 & 0.029 & 0.00780 & -0.00366 & 0.10606 & 0.0002 & 0.0032 & 0.0008 & 38 & 12.7 & 223 & 34.1 & 1075 & $8.66 \mathrm{E}-05$ & 48.9 \\
\hline 169 & 0.056 & 0.04073 & 0.01599 & 2.22570 & 0.0060 & 0.0100 & 0.0052 & 146 & 7.8 & 33 & 35.6 & 6201 & $8.70 \mathrm{E}-05$ & 48.4 \\
\hline 168 & 30.073 & 0.02614 & -0.00212 & 0.85625 & 0.0064 & 0.0214 & 0.0131 & 245 & 4.8 & 97 & 36.0 & 23264 & $8.75 \mathrm{E}-05$ & 47.1 \\
\hline 167 & 0.059 & 0.03290 & 0.01189 & 0.78393 & 0.0062 & 0.0096 & 0.0051 & 125 & 6.4 & 66 & 36.5 & 13331 & $8.80 \mathrm{E}-05$ & 47.5 \\
\hline 166 & 0.116 & 0.04320 & -0.00502 & 4.31238 & 0.0250 & 0.0142 & 0.0064 & 617 & 6.5 & 57 & 41.6 & 52928 & $8.84 \mathrm{E}-05$ & 49.7 \\
\hline 165 & 0.113 & 0.08378 & -0.00352 & 11.76866 & 0.0509 & 0.0204 & 0.0093 & 642 & 8.8 & 26 & 40.4 & 46880 & $8.89 \mathrm{E}-05$ & 48.3 \\
\hline 164 & 40.121 & 0.08789 & 0.00206 & 7.99624 & 0.0692 & 0.0283 & 0.0283 & 698 & 4.9 & 29 & 35.7 & 74874 & $8.94 \mathrm{E}-05$ & 48.1 \\
\hline 163 & 0.120 & 0.04086 & -0.01189 & 5.57620 & 0.0190 & 0.0138 & 0.0046 & 611 & 8.7 & 58 & 43.1 & 39925 & $8.99 \mathrm{E}-05$ & 47.6 \\
\hline 162 & 0.104 & 0.02731 & -0.01211 & 2.10615 & 0.0079 & 0.0154 & 0.0069 & 470 & 4.9 & 90 & 37.2 & 27272 & $9.04 \mathrm{E}-05$ & 47.5 \\
\hline 161 & 0.113 & 0.08278 & 0.03476 & 6.56774 & 0.0558 & 0.0426 & 0.0377 & 448 & 4.7 & 26 & 34.8 & 47680 & $9.08 \mathrm{E}-05$ & 50.0 \\
\hline 160 & 0.120 & 0.06800 & 0.02895 & 7.41137 & 0.0468 & 0.0180 & 0.0117 & 528 & 5.5 & 28 & 37.8 & 46090 & $9.13 \mathrm{E}-05$ & 47.4 \\
\hline 159 & 0.057 & 0.01641 & -0.00795 & 0.49631 & 0.0015 & 0.0142 & 0.0025 & 130 & 12.7 & 150 & 46.0 & 7229 & $9.18 \mathrm{E}-05$ & 46.3 \\
\hline 158 & 0.119 & 0.07251 & 0.03017 & $\begin{array}{l}.4 .60404 \\
\end{array}$ & 0.0485 & 0.0516 & 0.0346 & 511 & $\begin{array}{r}4.7 \\
\end{array}$ & 26 & 35.0 & 42154 & $9.24 \mathrm{E}-05$ & 47.9 \\
\hline 157 & 0.103 & 0.05571 & -0.02383 & 6.89424 & 0.0165 & 0.0068 & 0.0056 & 432 & 9.6 & 41 & 42.2 & 21921 & $9.29 \mathrm{E}-05$ & 48.3 \\
\hline 156 & 0.057 & 0.02496 & 0.00258 & 0.99957 & 0.0045 & 0.0217 & 0.0083 & 198 & 5.0 & $\begin{array}{l}41 \\
69\end{array}$ & 34.3 & 10543 & $9.34 \mathrm{E}-05$ & 47.4 \\
\hline 155 & 50.046 & 0.02041 & -0.00980 & 0.58187 & 0.0012 & 0.0127 & 0.0092 & 114 & 5.6 & 86 & 34.2 & 3271 & 9.39E-05 & 48.9 \\
\hline 154 & 0.104 & 0.06606 & -0.03045 & 4.59652 & 0.0209 & 0.0530 & 0.0073 & 369 & 8.6 & 46 & 42.4 & 32492 & $9.45 \mathrm{E}-05$ & 48.4 \\
\hline 153 & 0.083 & 0.02114 & 0.00991 & 0.96177 & 0.0066 & 0.0024 & 0.0071 & 227 & 4.6 & 85 & 34.0 & 17973 & $9.50 \mathrm{E}-05$ & 49.4 \\
\hline 152 & 20.054 & 0.03957 & -0.01615 & 2.00499 & 0.0032 & 0.0076 & 0.0044 & 134 & 9.0 & 45 & 37.0 & 4254 & $9.55 \mathrm{E}-05$ & 49.1 \\
\hline 151 & 0.071 & 0.04812 & -0.01786 & 1.59412 & 0.0068 & 0.0367 & $\begin{array}{l}0.0205 \\
0\end{array}$ & 193 & $\begin{array}{l}4.0 \\
4.9\end{array}$ & 51 & 34.6 & $\begin{array}{r}12444 \\
\end{array}$ & $9.61 \mathrm{E}-05$ & 47.7 \\
\hline 150 & 0.038 & 0.01003 & 0.00173 & 0.12474 & 0.0008 & 0.0078 & 0.0042 & 68 & 4.9 & 189 & 32.1 & 4570 & $9.67 \mathrm{E}-05$ & 49.8 \\
\hline 149 & 0.035 & 0.02452 & -0.00012 & 0.23282 & 0.0015 & 0.0034 & 0.0025 & 46 & 9.7 & 101 & 35.4 & 4482 & $9.72 \mathrm{E}-05$ & 48.3 \\
\hline 148 & 0.051 & 0.01308 & -0.00178 & 0.50862 & 0.0015 & 0.0116 & 0.0022 & 174 & 6.3 & 128 & 38.0 & 6815 & $9.78 \mathrm{E}-05$ & 48.0 \\
\hline 147 & 0.117 & 0.04746 & 0.01873 & 4.67730 & 0.0310 & 0.0078 & 0.0228 & 529 & 4.7 & 40 & 36.6 & 41808 & 9.84E-05 & 49.6 \\
\hline 146 & 0.118 & 0.05701 & -0.00646 & 4.97070 & 0.0371 & 0.0485 & 0.0061 & 563 & 9.3 & 54 & 43.9 & 75043 & $9.90 \mathrm{E}-05$ & 46.9 \\
\hline 145 & 0.072 & 0.04791 & 0.01624 & 2.86353 & 0.0112 & 0.0063 & 0.0211 & 226 & 4.9 & 32 & 33.7 & 11515 & $9.95 \mathrm{E}-05$ & 48.6 \\
\hline 144 & 0.082 & 0.02541 & 0.00826 & 2.94934 & 0.0089 & 0.0141 & 0.0026 & 369 & 9.4 & 59 & 41.1 & 17930 & $1.00 \mathrm{E}-04$ & 47.9 \\
\hline 143 & 0.109 & 0.04582 & 0.01146 & 4.88082 & 0.0250 & 0.0048 & 0.0066 & 491 & 6.5 & 42 & 40.7 & 36550 & $1.01 \mathrm{E}-04$ & 49.7 \\
\hline 142 & 0.059 & 0.01507 & 0.00449 & 0.79989 & 0.0029 & 0.0022 & 0.0019 & 186 & 7.6 & 99 & 36.6 & 8778 & $1.01 \mathrm{E}-04$ & 47.7 \\
\hline 141 & 0.106 & 0.05587 & -0.02755 & 8.87975 & 0.0148 & 0.0416 & 0.0086 & 518 & 6.4 & 33 & 37.0 & 16290 & $1.02 \mathrm{E}-04$ & 49.7 \\
\hline 140 & 0.057 & 0.01653 & -0.00800 & 0.61747 & 0.0015 & $\begin{array}{l}0.0145 \\
0\end{array}$ & 0.0017 & 145 & $\begin{array}{r}13.4 \\
13.0\end{array}$ & $\begin{array}{l}33 \\
136\end{array}$ & 45.7 & 6728 & $1.03 \mathrm{E}-04$ & 47.2 \\
\hline 139 & 0.101 & 0.03334 & -0.01016 & 2.08145 & 0.0130 & 0.0223 & 0.0122 & 495 & 4.7 & 80 & 37.4 & 40405 & $1.03 \mathrm{E}-04$ & 48.4 \\
\hline 138 & 0.120 & 0.05926 & 0.02257 & $\begin{array}{l}13.23444 \\
\end{array}$ & 0.0383 & 0.0430 & 0.0061 & 630 & 8.9 & 25 & 40.2 & 32631 & $1.04 \mathrm{E}-04$ & $\begin{array}{l}48.6 \\
\end{array}$ \\
\hline 137 & 0.057 & 0.01825 & -0.00593 & 0.41988 & 0.0021 & 0.0163 & 0.0040 & 135 & 9.1 & 146 & 43.3 & 10736 & $1.05 \mathrm{E}-04$ & 47.3 \\
\hline 136 & 0.043 & 0.03185 & 0.00852 & 0.53633 & 0.0027 & 0.0271 & 0.0148 & 72 & 4.9 & 55 & 32.1 & 4528 & $1.05 \mathrm{E}-04$ & 49.7 \\
\hline 135 & 0.055 & 0.01415 & $\begin{array}{l}-0.00655 \\
\end{array}$ & 0.29660 & 0.0011 & 0.0073 & 0.0058 & 95 & 11.8 & 183 & 40.3 & 6034 & $1.06 \mathrm{E}-04$ & $\begin{array}{l}47.1 \\
47.9\end{array}$ \\
\hline 134 & 0.057 & 0.01653 & -0.00813 & 0.61747 & 0.0015 & 0.0147 & 0.0026 & 145 & 13.0 & 135 & 46.1 & 6605 & $1.07 \mathrm{E}-04$ & 46.5 \\
\hline 133 & 0.101 & 0.02703 & -0.01314 & $\begin{array}{l}3.96872 \\
\end{array}$ & 0.0073 & 0.0216 & 0.0029 & 481 & $\begin{array}{r}1.0 \\
9.1\end{array}$ & 76 & $\begin{array}{l}40.1 \\
43.1\end{array}$ & 18491 & $1.07 \mathrm{E}-04$ & 47.7 \\
\hline 132 & 20.055 & 0.01595 & -0.00785 & 0.55499 & 0.0013 & 0.0141 & 0.0024 & 130 & 12.9 & 138 & 45.9 & 5706 & $1.08 \mathrm{E}-04$ & 48.1 \\
\hline 131 & 0.120 & 0.05664 & -0.02815 & 5.64219 & 0.0222 & 0.0227 & 0.0062 & 507 & 8.6 & 51 & 43.6 & 38929 & 1.09E-04 & 47.0 \\
\hline 130 & 0.070 & 0.02897 & -0.00195 & 2.57410 & 0.0062 & 0.0226 & 0.0057 & 356 & 5.0 & 50 & 34.6 & 10970 & $1.09 \mathrm{E}-04$ & 48.1 \\
\hline 129 & 0.106 & 0.07866 & -0.03388 & 5.67111 & 0.0268 & 0.0169 & 0.0387 & 475 & 4.9 & 34 & 35.3 & 33168 & 1.10E-04 & 48.0 \\
\hline 128 & 0.118 & 0.04858 & -0.00066 & 4.48461 & 0.0322 & 0.0271 & 0.0056 & 587 & 8.4 & 56 & 43.2 & 68473 & $1.11 \mathrm{E}-04$ & 49.8 \\
\hline 127 & 0.117 & 0.02977 & 0.01308 & 3.45957 & 0.0184 & 0.0215 & 0.0045 & 499 & 6.4 & 63 & 40.7 & 41559 & 1.12E-04 & 48.5 \\
\hline 126 & 0.047 & 0.01990 & -0.00805 & 0.31091 & 0.0014 & 0.0160 & 0.0087 & 84 & 10.7 & 139 & 42.7 & 6565 & $1.13 \mathrm{E}-04$ & 50.0 \\
\hline 125 & 50.063 & 0.02245 & -0.00976 & 0.84950 & 0.0028 & 0.0175 & 0.0112 & 189 & 7.9 & 102 & 41.0 & 9718 & $1.15 \mathrm{E}-04$ & 50.0 \\
\hline 124 & 0.121 & 0.05816 & -0.00648 & $\begin{array}{r}.0 .04300 \\
15.33762\end{array}$ & 0.0382 & 0.0060 & 0.0062 & 936 & 9.1 & 30 & 41.0 & 39737 & & \\
\hline 123 & 0.118 & 0.03259 & 0.00535 & $\begin{array}{r}3.10732 \\
\end{array}$ & 0.0240 & 0.0282 & 0.0039 & 572 & 8.3 & 78 & 43.8 & 69642 & & \\
\hline 122 & 0.117 & 0.04419 & 0.02076 & 6.19776 & 0.0313 & 0.0320 & 0.0056 & 567 & $\begin{array}{l}7.5 \\
7.4\end{array}$ & 41 & 41.6 & 44379 & & \\
\hline 121 & 0.064 & 0.04204 & -0.01581 & 1.53612 & 0.0058 & 0.0204 & 0.0047 & 169 & 8.6 & 59 & 40.0 & 10928 & & \\
\hline 120 & 0.116 & 0.03170 & -0.01458 & 3.72045 & $\begin{array}{l}0.0104 \\
0\end{array}$ & 0.0148 & 0.0032 & 463 & $\begin{array}{l}9.0 \\
9.4\end{array}$ & 81 & 43.8 & 28635 & & \\
\hline 119 & 0.107 & 0.02769 & -0.00035 & 2.29974 & 0.0146 & 0.0243 & 0.0028 & 473 & 9.8 & 98 & 45.2 & 55388 & & \\
\hline 118 & 0.081 & 0.03693 & -0.01767 & 2.34704 & 0.0070 & 0.0224 & 0.0039 & 266 & 9.3 & 69 & 41.7 & 15911 & & \\
\hline 117 & 0.028 & 0.01540 & -0.00608 & 0.09734 & 0.0004 & 0.0138 & 0.0077 & 29 & $\begin{array}{r}10.6 \\
\end{array}$ & 167 & 39.0 & 1971 & & \\
\hline 116 & 0.121 & 0.05749 & -0.02246 & 7.80297 & 0.0285 & 0.0092 & 0.0084 & 735 & 6.5 & 41 & 40.7 & 41834 & & \\
\hline 115 & 0.118 & 0.07459 & 0.01107 & 6.88964 & 0.0472 & 0.0497 & 0.0080 & 469 & 9.3 & 35 & 42.3 & 60043 & & \\
\hline 114 & 0.073 & 0.03758 & -0.01655 & 3.45041 & 0.0051 & 0.0125 & 0.0042 & 247 & 8.7 & 47 & 39.3 & 7345 & & \\
\hline 113 & 0.106 & 0.03016 & -0.01390 & $\begin{array}{l}2.38157 \\
\end{array}$ & 0.0095 & 0.0245 & 0.0036 & 426 & 8.3 & 91 & 44.1 & 30449 & & \\
\hline 112 & 0.108 & 0.05169 & -0.02514 & 4.16365 & 0.0145 & 0.0060 & 0.0193 & 480 & 4.7 & 46 & 36.6 & 25214 & & \\
\hline 111 & 0.068 & 0.04621 & -0.01273 & 2.43072 & 0.0086 & 0.0412 & 0.0047 & 227 & 9.3 & 49 & 40.9 & 14003 & & \\
\hline 110 & 0.112 & 0.08144 & 0.02116 & 7.90144 & 0.0534 & 0.0698 & 0.0206 & 553 & 4.7 & 25 & 34.6 & 46032 & & \\
\hline 109 & 0.090 & 0.06553 & -0.03065 & 4.54543 & 0.0130 & 0.0274 & 0.0079 & 267 & 8.2 & 39 & 38.7 & 16131 & & \\
\hline 108 & 30.116 & 0.06442 & -0.02020 & 5.42793 & 0.0275 & 0.0562 & 0.0067 & 433 & 9.4 & 48 & 43.1 & 46329 & $1.37 \mathrm{E}-04$ & 48.2 \\
\hline 107 & 0.090 & 0.02314 & -0.00097 & 1.24506 & 0.0097 & 0.0050 & 0.0042 & 392 & 5.4 & 104 & 36.7 & 34587 & $1.37 \mathrm{E}-04$ & 48.2 \\
\hline 106 & 0.101 & 0.03887 & -0.01940 & 2.56119 & 0.0105 & 0.0045 & 0.0061 & 393 & 6.3 & 71 & 40.1 & 26626 & 1.38E-04 & 49.8 \\
\hline
\end{tabular}




\begin{tabular}{|c|c|c|c|c|c|c|c|c|c|c|c|c|c|c|}
\hline Run \# & $\begin{array}{l}\text { Bore } \\
(\mathrm{m})\end{array}$ & $\begin{array}{l}\text { Stroke eff. } \\
(\mathrm{m})\end{array}$ & $\begin{array}{l}\text { Free Travel } \\
\text { (m) }\end{array}$ & $\begin{array}{l}\text { Mass } \\
(\mathrm{kg})\end{array}$ & $\begin{array}{l}\text { Fuel injected } \\
(\mathrm{ml})\end{array}$ & $\begin{array}{l}\text { Injection timing } \\
\text { (mm before TDC) }\end{array}$ & $\begin{array}{l}\text { Ignition timing } \\
\text { (mm before TDC) }\end{array}$ & $\begin{array}{l}\text { Force on shaft } \\
(\mathrm{N})\end{array}$ & Compr. ratio & $\begin{array}{l}\text { Op. frequency } \\
(\mathrm{Hz})\end{array}$ & $\begin{array}{l}\text { Sim. efficiency } \\
(\%)\end{array}$ & $\begin{array}{l}\text { Power output } \\
\text { (W) }\end{array}$ & NN perform. & $\begin{array}{l}\text { Predicted eff. } \\
\text { (\%) }\end{array}$ \\
\hline 105 & 0.095 & 0.06874 & 0.02752 & 7.85955 & $\quad 0.0287$ & 0.0288 & 0.0069 & 346 & 10.0 & 25 & 38.6 & $6 \quad 24058$ & $1.39 \mathrm{E}-04$ & 48.2 \\
\hline 104 & 0.117 & 0.03636 & 0.01094 & 3.52292 & 0.0278 & 0.0071 & 0.0037 & 523 & 9.7 & 67 & 43.5 & 65977 & $1.41 \mathrm{E}-04$ & 47.0 \\
\hline 103 & 0.102 & 0.05372 & -0.02471 & 3.60096 & 0.0135 & 0.0074 & 0.0065 & 325 & 7.8 & 52 & 41.4 & 23199 & $1.42 \mathrm{E}-04$ & 47.9 \\
\hline 102 & 0.118 & 0.04021 & -0.02003 & 6.87858 & 0.0131 & 0.0204 & 0.0195 & 742 & 4.8 & 45 & 36.2 & 21858 & 1.43E-04 & 49.8 \\
\hline 101 & 0.111 & 0.05410 & 0.02071 & 5.07916 & 0.0374 & 0.0387 & 0.0226 & 518 & 4.9 & 38 & 35.7 & 48998 & 1.44E-04 & 49.0 \\
\hline 100 & 0.027 & 0.01732 & -0.00714 & 0.18325 & 0.0004 & 0.0148 & 0.0030 & 34 & 7.8 & 103 & 33.9 & 1077 & $1.45 \mathrm{E}-04$ & 48.2 \\
\hline 99 & 0.027 & 0.01732 & -0.00714 & 0.18325 & 0.0004 & 0.0148 & 0.0030 & 34 & 7.8 & 103 & 33.9 & 1077 & 1.47E-04 & 45.0 \\
\hline 98 & 0.073 & 0.02137 & -0.00061 & 1.78788 & 0.0050 & 0.0172 & 0.0098 & 357 & 4.9 & 71 & 35.4 & 12647 & 1.48E-04 & 47.5 \\
\hline 97 & 0.068 & 0.01971 & -0.00162 & 1.63475 & 0.0039 & 0.0118 & 0.0097 & 333 & 5.0 & 73 & 34.9 & 10108 & 1.50E-04 & 48.0 \\
\hline 96 & 0.085 & 0.02336 & -0.00148 & 2.54230 & 0.0072 & 0.0174 & 0.0115 & 480 & 4.9 & 68 & 35.8 & 17987 & $1.51 \mathrm{E}-04$ & 47.4 \\
\hline 95 & 0.102 & 0.05372 & -0.02471 & 3.60096 & 0.0135 & 0.0074 & 0.0065 & 325 & 7.8 & 52 & 41.4 & 23199 & $1.52 \mathrm{E}-04$ & 47.8 \\
\hline 94 & 0.118 & 0.02959 & -0.00806 & 6.28101 & 0.0136 & 0.0058 & 0.0042 & 816 & 6.7 & 57 & 41.2 & 27387 & 1.53E-04 & 48.1 \\
\hline 93 & 0.085 & 0.03020 & 0.01478 & 3.81625 & 0.0119 & 0.0036 & 0.0151 & 428 & 5.0 & 41 & 34.9 & 15936 & $1.55 \mathrm{E}-04$ & 48.2 \\
\hline 92 & 0.064 & 0.01673 & 0.00805 & 0.47773 & 0.0037 & 0.0150 & 0.0037 & 151 & 5.0 & 118 & 35.4 & 14430 & & \\
\hline 91 & 0.117 & 0.08656 & -0.00913 & 7.57619 & 0.0596 & 0.0668 & 0.0091 & 582 & 9.2 & 35 & 42.6 & 75570 & & \\
\hline 90 & 0.015 & 0.00374 & -0.00063 & 0.01384 & 0.0000 & 0.0025 & 0.0019 & 10 & 6.8 & 379 & 25.3 & 260 & & \\
\hline 89 & 0.011 & 0.00329 & 0.00105 & 0.00704 & 0.0000 & 0.0027 & 0.0013 & 4 & 5.1 & 348 & 21.7 & 122 & & \\
\hline 88 & 0.011 & 0.00329 & 0.00105 & 0.00704 & 0.0000 & 0.0027 & 0.0013 & 4 & 5.2 & 348 & 21.7 & 122 & & \\
\hline 87 & 0.121 & 0.04407 & 0.01463 & 11.80406 & 0.0359 & 0.0096 & 0.0081 & 984 & 5.0 & 29 & 36.9 & 35543 & & \\
\hline 86 & 0.022 & 0.00889 & -0.00437 & 0.03225 & 0.0001 & 0.0009 & 0.0044 & 8 & 13.2 & 256 & 20.8 & 358 & & \\
\hline 85 & 0.022 & 0.00889 & -0.00437 & 0.03225 & 0.0001 & 0.0009 & 0.0044 & 8 & 13.1 & 256 & 20.8 & 358 & & \\
\hline 84 & 0.121 & 0.07368 & 0.03367 & 19.69064 & 0.0531 & 0.0652 & 0.0085 & 700 & 8.1 & 18 & 36.4 & 30974 & $1.74 \mathrm{E}-04$ & 46.6 \\
\hline 83 & 0.101 & 0.04696 & -0.02137 & 8.68588 & 0.0139 & 0.0409 & 0.0081 & 675 & 5.5 & 34 & 36.7 & 16453 & $1.76 \mathrm{E}-04$ & 46.0 \\
\hline 82 & 0.102 & 0.06891 & -0.02873 & 13.17204 & 0.0225 & 0.0610 & 0.0204 & 10 & 10.0 & 200 & 10.0 & 1 & $1.78 \mathrm{E}-04$ & 47.3 \\
\hline 81 & 0.118 & 0.05436 & -0.02123 & 13.42212 & 0.0251 & 0.0439 & 0.0102 & 1013 & 5.0 & 29 & 36.1 & 25992 & $1.80 \mathrm{E}-04$ & 47.8 \\
\hline 80 & 0.117 & 0.03582 & -0.00430 & 8.71399 & 0.0242 & 0.0290 & 0.0069 & 1159 & 4.9 & 43 & 37.4 & 38096 & $1.82 \mathrm{E}-04$ & \\
\hline 79 & 0.115 & 0.08577 & -0.03736 & 13.38913 & 0.0357 & 0.0748 & 0.0088 & 569 & 9.6 & 27 & 38.9 & 30563 & $1.84 \mathrm{E}-04$ & 47.2 \\
\hline 78 & 0.011 & 0.00422 & 0.00172 & 0.00833 & 0.0000 & 0.0038 & 0.0005 & 4 & 8.3 & 329 & 25.6 & 188 & $1.86 \mathrm{E}-04$ & 46.5 \\
\hline 77 & 0.099 & 0.02969 & 0.01458 & 5.17259 & 0.0130 & 0.0255 & 0.0034 & 445 & 8.5 & 44 & 40.2 & 19135 & & \\
\hline 76 & 0.109 & 0.07993 & 0.03872 & 6.11642 & 0.0508 & 0.0084 & 0.0099 & 380 & 7.9 & 29 & 40.5 & 50827 & $1.91 \mathrm{E}-04$ & 48.3 \\
\hline 75 & 0.024 & 0.00635 & -0.00297 & 0.02463 & 0.0001 & 0.0009 & 0.0028 & 9 & 12.4 & 372 & 17.8 & 488 & $1.93 \mathrm{E}-04$ & 45.8 \\
\hline 74 & 0.013 & 0.00516 & -0.00235 & 0.00938 & 0.0000 & 0.0044 & 0.0007 & 5 & 10.0 & 200 & 10.0 & 1 & $1.95 \mathrm{E}-04$ & 49.3 \\
\hline 73 & 0.070 & 0.02392 & -0.01163 & 1.95617 & 0.0029 & 0.0211 & 0.0026 & 220 & 9.3 & 79 & 40.2 & 7153 & $1.97 \mathrm{E}-04$ & 48.1 \\
\hline 72 & 0.111 & 0.07767 & -0.01911 & 6.47854 & 0.0370 & 0.0102 & 0.0078 & 471 & 9.7 & 38 & 43.3 & 49780 & $2.00 \mathrm{E}-04$ & 47.9 \\
\hline 71 & 0.036 & 0.00948 & 0.00441 & 0.22405 & 0.0005 & 0.0085 & 0.0025 & 63 & 5.0 & 122 & 32.2 & 2029 & $2.02 \mathrm{E}-04$ & 48.1 \\
\hline 70 & 0.098 & 0.06089 & -0.02973 & 10.70917 & 0.0163 & 0.0100 & 0.0061 & 480 & 9.5 & 30 & 39.4 & 15058 & 2.05E-04 & 49.1 \\
\hline 69 & 0.082 & 0.02101 & -0.00692 & 2.45938 & 0.0053 & 0.0085 & 0.0087 & 533 & 4.8 & 75 & 36.3 & 14645 & $2.07 \mathrm{E}-04$ & \\
\hline 68 & 0.121 & 0.03754 & -0.01749 & 9.32743 & 0.0154 & 0.0122 & 0.0042 & 800 & 8.5 & 49 & 42.6 & 25713 & 2.10E-04 & 48.5 \\
\hline 67 & 0.099 & 0.07377 & -0.02168 & 12.14881 & 0.0280 & 0.0143 & 0.0107 & 601 & 6.9 & 24 & 36.0 & 21641 & $2.13 \mathrm{E}-04$ & 46.3 \\
\hline 66 & 0.103 & 0.02902 & 0.01397 & 5.55619 & 0.0153 & 0.0043 & 0.0031 & 533 & 9.1 & 48 & 42.2 & 25040 & $2.15 \mathrm{E}-04$ & 47.6 \\
\hline 65 & 0.115 & 0.04263 & -0.01707 & 6.65511 & 0.0188 & 0.0372 & 0.0046 & 667 & 8.9 & 53 & 43.3 & 34975 & $2.18 \mathrm{E}-04$ & 48.4 \\
\hline 64 & 0.075 & 0.03034 & 0.01487 & 2.10890 & 0.0095 & 0.0234 & 0.0031 & 247 & 9.3 & 57 & 41.4 & 18513 & 2.21E-04 & 45.9 \\
\hline 63 & 0.010 & 0.00293 & -0.00144 & 0.00252 & 0.0000 & 0.0006 & 0.0007 & 30 & 10.0 & 200 & 10.0 & 1 & 2.24E-04 & 46.0 \\
\hline 62 & 0.022 & 0.00884 & 0.00385 & 0.02790 & 0.0002 & 0.0019 & 0.0040 & 10 & 5.0 & 199 & 18.5 & 745 & 2.27E-04 & 47.0 \\
\hline 61 & 0.013 & 0.00924 & 0.00171 & 0.02812 & 0.0001 & 0.0076 & 0.0011 & 7 & 8.0 & 157 & 26.3 & 284 & 2.30E-04 & 49.6 \\
\hline 60 & 0.052 & 0.01431 & -0.00688 & 0.34258 & 0.0009 & 0.0019 & 0.0021 & 82 & 12.7 & 157 & 37.7 & 3794 & $2.34 \mathrm{E}-04$ & 47.5 \\
\hline 59 & 0.011 & 0.00275 & -0.00098 & 0.00565 & 0.0000 & 0.0008 & 0.0007 & 20 & 10.0 & 200 & 10.0 & 1 & $2.37 \mathrm{E}-04$ & 49.1 \\
\hline 58 & 0.086 & 0.06313 & 0.00943 & 3.83339 & 0.0262 & 0.0462 & 0.0288 & 371 & 4.7 & 33 & 34.8 & 29500 & 2. $40 \mathrm{E}-04$ & 49.6 \\
\hline 57 & 0.011 & 0.00485 & 0.00239 & 0.00370 & 0.0000 & 0.0030 & 0.0024 & -9 & 10.0 & 200 & 10.0 & 1 & 2.44E-04 & 45.3 \\
\hline 56 & 0.117 & 0.07316 & 0.03308 & 18.11543 & 0.0450 & 0.0118 & 0.0330 & 679 & 4.8 & 16 & 31.7 & 23039 & $2.48 \mathrm{E}-04$ & 48.3 \\
\hline 55 & 0.085 & 0.02267 & -0.00914 & 1.01007 & 0.0055 & 0.0008 & 0.0111 & 273 & 10.4 & 124 & 43.0 & 22109 & $2.51 \mathrm{E}-04$ & 47.6 \\
\hline 54 & 0.048 & 0.01289 & -0.00541 & 0.44240 & 0.0009 & 0.0111 & 0.0062 & 117 & 11.0 & 143 & 43.3 & 4010 & $2.55 \mathrm{E}-04$ & 47.5 \\
\hline 53 & 0.011 & 0.00327 & 0.00046 & 0.00677 & 0.0000 & 0.0004 & 0.0016 & 2 & 10.0 & 100 & 10.0 & 0 & $2.59 \mathrm{E}-04$ & 48.3 \\
\hline 52 & 0.120 & 0.03241 & -0.01481 & 4.14549 & 0.0138 & 0.0272 & 0.0112 & 770 & 4.8 & 70 & 37.5 & 37432 & 2.63E-04 & 49.3 \\
\hline 51 & 0.055 & 0.03717 & 0.00091 & 0.97616 & 0.0059 & 0.0286 & 0.0040 & 139 & 8.6 & 64 & 40.3 & 13024 & $2.68 \mathrm{E}-04$ & 46.1 \\
\hline 50 & 0.116 & 0.04237 & 0.00453 & 6.87811 & 0.0311 & 0.0301 & 0.0120 & 847 & 4.9 & 41 & 36.0 & 45961 & $2.72 \mathrm{E}-04$ & 49.6 \\
\hline 49 & 0.009 & 0.00401 & 0.00073 & 0.00239 & 0.0000 & 0.0024 & 0.0014 & 1 & 5.1 & 442 & 8.9 & 54 & $2.77 \mathrm{E}-04$ & 45.4 \\
\hline 48 & 0.079 & 0.03456 & 0.00260 & 2.54036 & 0.0098 & 0.0042 & 0.0078 & 345 & 5.0 & 50 & 35.9 & 17991 & $2.81 \mathrm{E}-04$ & 41.9 \\
\hline 47 & 0.085 & 0.03046 & 0.01033 & 3.07974 & 0.0103 & 0.0081 & 0.0104 & 375 & 4.8 & 45 & 35.3 & 15290 & $2.86 \mathrm{E}-04$ & 45.3 \\
\hline 46 & 0.117 & 0.07179 & -0.00632 & 14.99839 & 0.0428 & 0.0324 & 0.0340 & 906 & 4.8 & 22 & 33.9 & 33037 & $2.91 \mathrm{E}-04$ & 48.6 \\
\hline 45 & 0.120 & 0.07763 & -0.00476 & 13.78121 & 0.0498 & 0.0477 & 0.0177 & 851 & 5.0 & 24 & 33.7 & 42981 & $2.96 \mathrm{E}-04$ & 48.5 \\
\hline 44 & 0.013 & 0.00610 & -0.00119 & 0.01826 & 0.0000 & 0.0044 & 0.0030 & 7 & 7.2 & 241 & 26.0 & 211 & & \\
\hline 43 & 0.097 & 0.05721 & -0.00223 & 7.02819 & 0.0277 & 0.0084 & 0.0195 & 656 & 4.8 & 30 & 35.0 & 29347 & & \\
\hline 42 & 0.033 & 0.01801 & 0.00469 & 0.25287 & 0.0010 & 0.0048 & 0.0045 & 54 & 5.1 & 84 & 30.6 & 2530 & & \\
\hline 41 & 0.015 & 0.01115 & 0.00092 & 0.02833 & 0.0001 & 0.0041 & 0.0030 & 9 & 5.2 & 149 & 22.5 & 416 & & \\
\hline 40 & 0.009 & 0.00623 & -0.00187 & 0.00487 & 0.0000 & 0.0020 & 0.0018 & 1 & 8.9 & 305 & 11.5 & 51 & & \\
\hline 39 & 0.027 & 0.02021 & -0.00462 & 0.13902 & 0.0006 & 0.0139 & 0.0031 & 34 & 7.0 & 109 & 32.7 & 2067 & & \\
\hline 38 & 0.058 & 0.03483 & 0.00423 & 1.87921 & 0.0066 & 0.0180 & 0.0157 & 227 & 5.1 & 43 & 33.2 & 9052 & & \\
\hline 37 & 0.090 & 0.03662 & 0.01239 & 3.92378 & 0.0146 & 0.0242 & 0.0117 & 421 & 4.8 & 40 & 35.2 & 20041 & & \\
\hline 36 & 0.081 & 0.05346 & 0.00857 & 3.62885 & 0.0169 & 0.0199 & 0.0168 & 325 & 4.8 & 33 & 34.4 & 19312 & & \\
\hline 35 & 0.051 & 0.03450 & 0.01220 & 1.18982 & 0.0045 & 0.0283 & 0.0148 & 125 & 5.1 & 42 & 31.8 & 5768 & & \\
\hline 34 & 0.029 & 0.01013 & 0.00185 & 0.08508 & 0.0004 & $\begin{array}{l}0.0022 \\
0.0166\end{array}$ & 0.0038 & $\begin{array}{r}36 \\
422\end{array}$ & $\begin{array}{l}4.6 \\
4.2\end{array}$ & $\begin{array}{r}161 \\
43\end{array}$ & 26.5 & 1836 & & \\
\hline & & & & & & & 0.0083 & 422 & 4.2 & & & & & \\
\hline
\end{tabular}




\begin{tabular}{|c|c|c|c|c|c|c|c|c|c|c|c|c|}
\hline Run \# Bore & $\begin{array}{l}\text { Stroke eff. } \\
(\mathrm{m})\end{array}$ & $\begin{array}{l}\text { Free Travel } \\
(\mathrm{m})\end{array}$ & $\begin{array}{l}\text { Mass } \\
(\mathrm{kg})\end{array}$ & $\begin{array}{l}\text { Fuel injected } \\
(\mathrm{ml})\end{array}$ & $\begin{array}{l}\text { Injection timing } \\
\text { (mm before TDC) }\end{array}$ & $\begin{array}{l}\text { Ignition timing } \\
\text { (mm before TDC) }\end{array}$ & $\begin{array}{l}\text { Force on shaft } \\
\text { (N) }\end{array}$ & Compr. ratio & $\begin{array}{l}\text { Op. frequency } \\
(\mathrm{Hz})\end{array}$ & $\begin{array}{l}\text { Sim. efficiency } \\
(\%)\end{array}$ & $\begin{array}{l}\text { Power output NN perform. } \\
\text { (W) }\end{array}$ & $\begin{array}{l}\text { Predicted eff. } \\
(\%)\end{array}$ \\
\hline 320.055 & 0.03724 & 0.00094 & 0.98136 & $\quad 0.0060$ & $\quad 0.0287$ & 0.0040 & 145 & 8.1 & 64 & 40.6 & $6 \quad 13504$ & \\
\hline $31 \quad 0.039$ & 0.01350 & -0.00654 & 0.30768 & 0.0005 & 0.0114 & 0.0039 & 69 & 12.5 & 139 & 42.6 & 2234 & \\
\hline 300.048 & 0.03140 & -0.01539 & 0.56228 & 0.0017 & 0.0081 & 0.0107 & 99 & 4.7 & 71 & 33.4 & 4456 & \\
\hline 290.028 & 0.01254 & 0.00546 & 0.16699 & 0.0005 & 0.0102 & 0.0015 & 34 & 7.7 & 107 & 33.4 & 1449 & \\
\hline 280.010 & 0.00642 & -0.00035 & 0.00827 & 0.0000 & 0.0054 & 0.0025 & 4 & 5.8 & 258 & 22.1 & 164 & \\
\hline $27 \quad 0.116$ & 0.04237 & 0.00453 & 6.87811 & 0.0311 & 0.0301 & 0.0120 & 937 & 4.1 & 41 & 38.0 & 48997 & \\
\hline 260.033 & 0.01801 & 0.00469 & 0.25287 & 0.0010 & 0.0048 & 0.0045 & 58 & 4.6 & 82 & 31.3 & 2599 & \\
\hline 250.015 & 0.01115 & 0.00092 & 0.02833 & 0.0001 & 0.0041 & 0.0030 & 9 & 4.9 & 152 & 23.3 & 460 & \\
\hline 240.009 & 0.00623 & -0.00187 & 0.00487 & 0.0000 & 0.0020 & 0.0018 & 1 & 8.7 & 307 & 11.7 & 53 & \\
\hline 230.027 & 0.02021 & -0.00462 & 0.13902 & 0.0006 & 0.0139 & 0.0031 & 34 & 6.8 & 111 & 32.9 & 2140 & \\
\hline 220.058 & 0.03483 & 0.00423 & 1.87921 & 0.0066 & 0.0180 & 0.0157 & 234 & 4.9 & 46 & 33.7 & 10678 & \\
\hline 210.090 & 0.03662 & 0.01239 & 3.92378 & 0.0146 & 0.0242 & 0.0117 & 449 & 5.0 & 43 & 34.9 & 23940 & \\
\hline 200.081 & 0.05346 & 0.00857 & 3.62885 & 0.0169 & 0.0199 & 0.0168 & 341 & 4.5 & 34 & 35.1 & 21427 & \\
\hline 190.051 & 0.03450 & 0.01220 & 1.18982 & 0.0045 & 0.0283 & 0.0148 & 137 & 4.8 & 43 & 32.1 & 6559 & \\
\hline $\begin{array}{ll}18 & 0.029\end{array}$ & 0.01013 & 0.00185 & 0.08508 & 0.0004 & 0.0022 & 0.0038 & 36 & 4.8 & 164 & 26.6 & 1885 & \\
\hline $\begin{array}{ll}17 & 0.085\end{array}$ & 0.03749 & 0.01244 & 3.34929 & 0.0143 & 0.0166 & 0.0083 & 422 & 4.7 & 43 & 35.5 & 23433 & \\
\hline $\begin{array}{ll}16 & 0.055\end{array}$ & 0.03724 & 0.00094 & 0.98136 & 0.0060 & 0.0287 & 0.0040 & 146 & 8.4 & 66 & 39.8 & 14543 & \\
\hline $\begin{array}{ll}15 & 0.039\end{array}$ & 0.01350 & -0.00654 & 0.30768 & 0.0005 & 0.0114 & 0.0039 & 70 & 12.5 & 138 & 42.5 & 2210 & \\
\hline $14 \quad 0.028$ & 0.01254 & 0.00546 & 0.16699 & 0.0005 & 0.0102 & 0.0015 & 33 & 8.1 & 105 & 33.4 & 1345 & \\
\hline $\begin{array}{ll}13 & 0.010\end{array}$ & 0.00642 & -0.00035 & 0.00827 & 0.0000 & 0.0054 & 0.0025 & 5 & 5.1 & 257 & 22.9 & 185 & \\
\hline $12 \quad 0.116$ & 0.04237 & 0.00453 & 6.87811 & 0.0311 & 0.0301 & 0.0120 & 838 & 5.0 & 40 & 36.3 & 42918 & \\
\hline 110.010 & 0.00642 & -0.00035 & 0.00827 & 0.0000 & 0.0054 & 0.0025 & 4 & 5.8 & 260 & 22.0 & 169 & \\
\hline $\begin{array}{lll}10 & 0.116\end{array}$ & 0.04237 & 0.00453 & 6.87811 & 0.0311 & 0.0301 & 0.0120 & 838 & 5.0 & 40 & 36.2 & 42884 & \\
\hline 90.051 & 0.03450 & 0.01220 & 1.18982 & 0.0045 & 0.0283 & 0.0148 & 122 & 5.1 & 42 & 31.9 & 5719 & \\
\hline 80.085 & 0.03749 & 0.01244 & 3.34929 & 0.0143 & 0.0166 & 0.0083 & 374 & 5.0 & 40 & 34.9 & 18400 & \\
\hline 70.055 & 0.03724 & 0.00094 & 0.98136 & 0.0060 & 0.0287 & 0.0040 & 134 & 9.3 & 65 & 39.7 & 12888 & \\
\hline $\begin{array}{ll}60.048 \\
\end{array}$ & 0.03140 & -0.01539 & 0.56228 & 0.0017 & 0.0081 & 0.0107 & 90 & 4.8 & 71 & 33.0 & 4071 & \\
\hline $\begin{array}{ll}50.028\end{array}$ & 0.01254 & 0.00546 & 0.16699 & 0.0005 & 0.0102 & 0.0015 & 36 & 7.7 & 108 & 35.2 & 1557 & \\
\hline 40.116 & 0.04237 & 0.00453 & 6.87811 & 0.0311 & 0.0301 & 0.0120 & 831 & 5.0 & 39 & 35.5 & 41001 & \\
\hline 30.114 & 0.04752 & 0.00739 & 6.72550 & 0.0318 & 0.0313 & 0.0123 & 705 & 4.9 & 35 & 35.2 & 37000 & \\
\hline 20.091 & 0.03156 & 0.01278 & 3.42113 & 0.0135 & 0.0091 & 0.0101 & 407 & 4.9 & 43 & 32.9 & 16952 & \\
\hline 10.055 & 0.03477 & 0.01022 & 1.86068 & 0.0058 & 0.0137 & 0.0121 & 183 & 5.1 & 35 & 32.1 & 5863 & \\
\hline
\end{tabular}




\section{Appendix C}

Appendix C shows the Matlab programs that were used for the simulation, prediction and optimization.

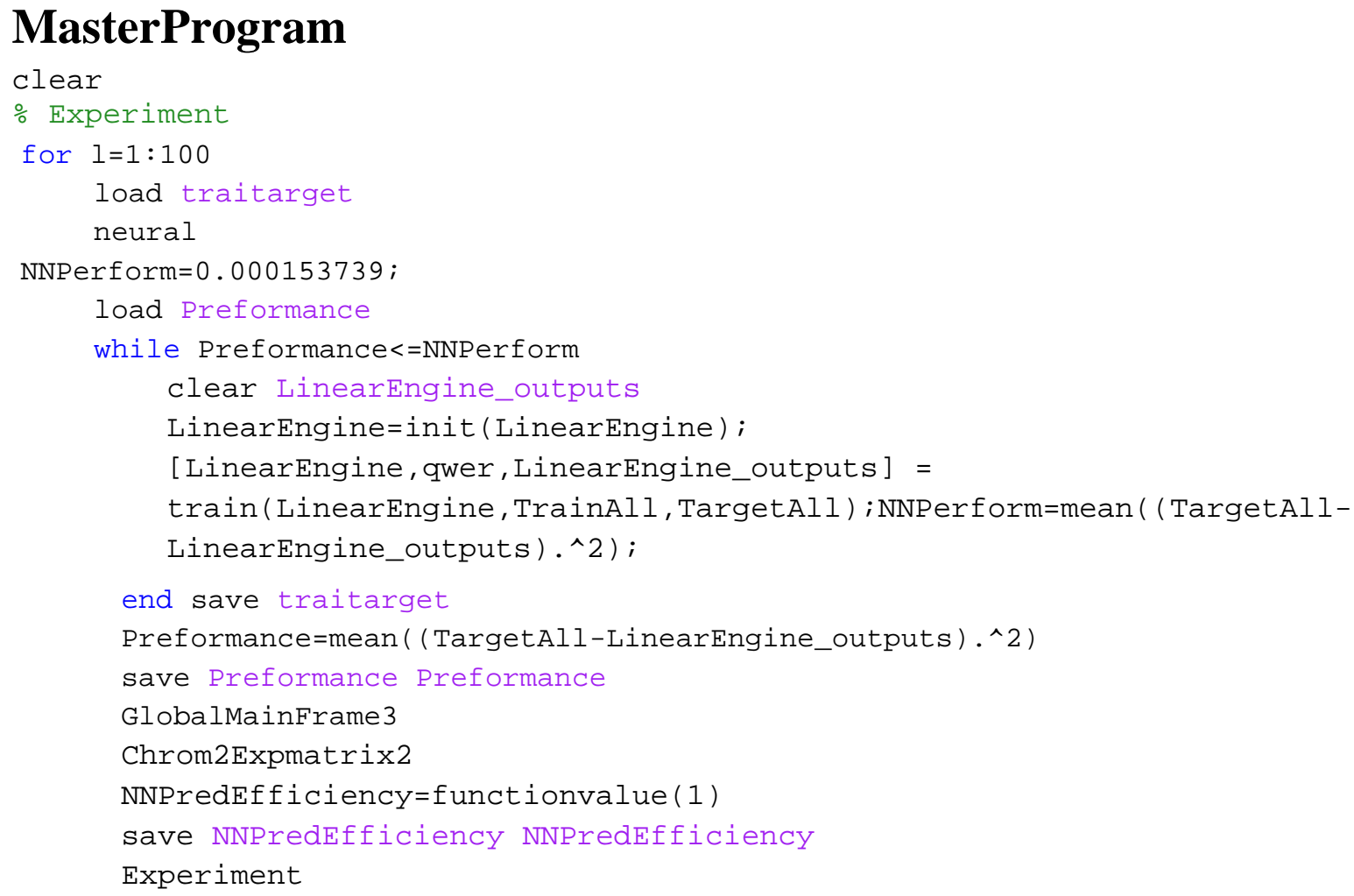

end

\section{Neural}

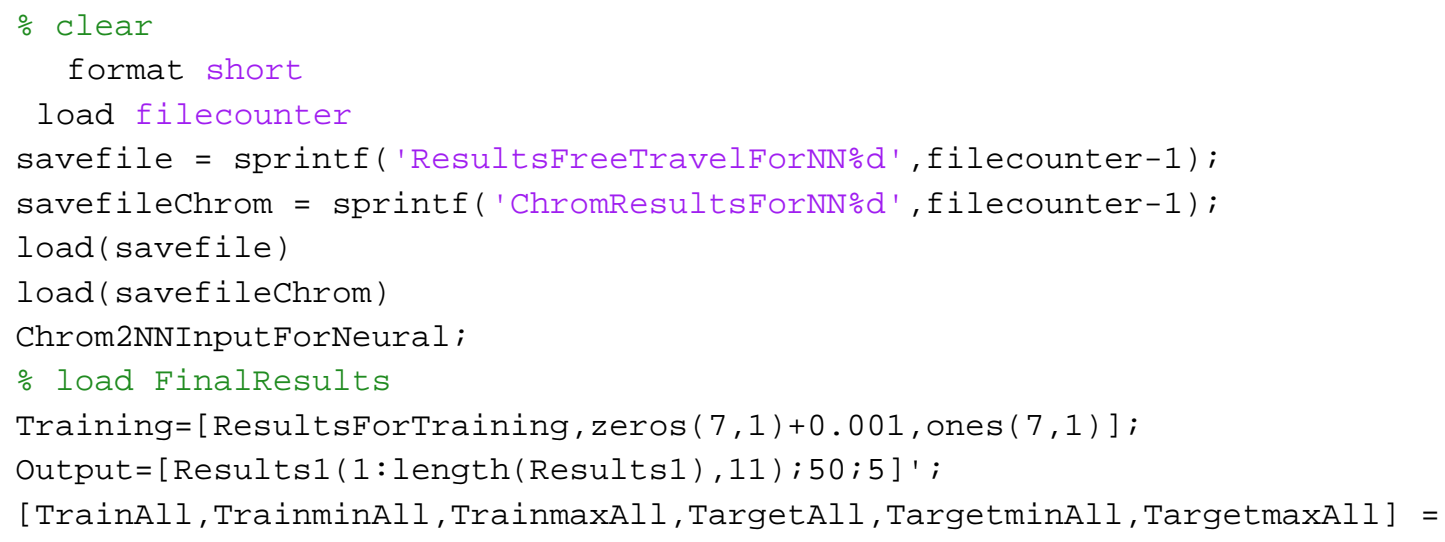


premnmx(Training, output);

TrainAll=TrainAll ( $:, 1:$ length (TrainAll) -2$)$;

TargetAll=TargetAll( : , 1: length (TargetAll) -2);

save traitargetneural

nntool

\section{GlobalMainFrame3}

Preformance=mean ( (TargetAll-LinearEngine_outputs).^2);

save Preformance Preformance

clear

$\% \% \%$ Load the Chromosomes (prerun)

format short $\mathrm{g}$

load traitarget

load filecounter

savefilechrom $=\operatorname{sprintf}($ 'ChromResultsForNN\%d', filecounter -1$)$;

savefileTarget $=$ sprintf('TargetResultsForTraining\%d', filecounter-1);

load (savefileTarget)

load (savefilechrom);

chromosom=ChromResults;

functionvalue=TargetResultsForTraining

functionvalueold=functionvalue;

[raws, columns]=size (chromosom);

$\mathrm{zs}=0$;

Chromstore $=\operatorname{chromosom}(1,:)$;

functionvaluestore=functionvalue(1);

Expold=zeros $(1,7)$;

expmatrix=zeros $(1,7)$;

$\% \% \%$ Run untill first chraomosome == last chromosome

$\%$ while or (or ( (bin2dec(chromosom(1,1:35))-

bin2dec (chromosom (raws, 1:35)) )>0, (bin2dec

$(\operatorname{chromosom}(1,36: 70))-\operatorname{bin} 2 \operatorname{dec}(\operatorname{chromosom}(\operatorname{raws}, 36: 70)))>0)$,

or ( (bin2dec (chromosom $(1,1: 35))$ -

bin2dec $(\operatorname{chromosom}(\operatorname{raws}, 1: 35)))<0, \quad(\operatorname{bin} 2 \operatorname{dec}(\operatorname{chromosom}(1,36: 70))-$

bin2dec $(\operatorname{chromosom}($ raws, $36: 70)))<0))$;

$z s=z s+1$

for $i=1: 100 \% \% \% \%$ GA Rest

$\% \% \%$ Run the NN on the new values that GA created to get the "functionvalue"

for $\mathrm{e}=1$ : raws Chromosome=chromosom $(\mathrm{e}, \mathrm{:})$; Chrom2NNInput; $\%$

ScaledNNInput $=(($ ResultsForTraining $(:, 1)$-TrainminAll $) . /($ TrainmaxAll -

TrainminAll) )*2-1;

Simresult=sim(LinearEngine, ScaledNNInput) ; Results (e)=postmnmx (Simresult, $T$

argetminAll, TargetmaxAll);

end

functionvalue=Results;

$\% \% \%$ GA

GeneticAlgorithmInitial 


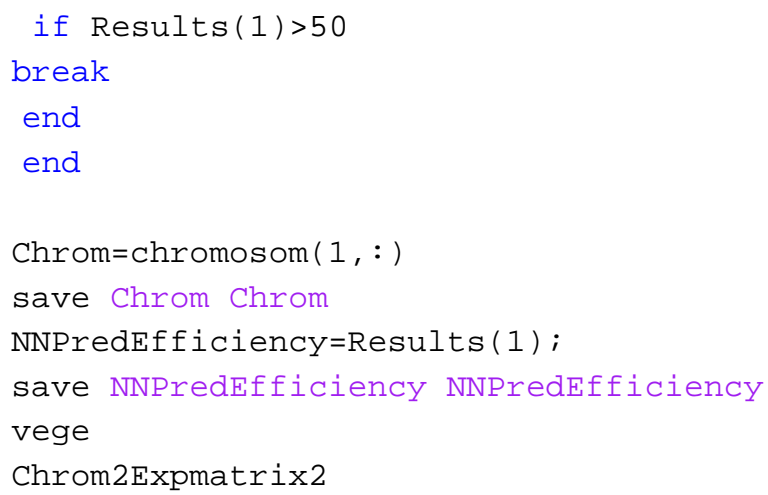

\section{Experiment}

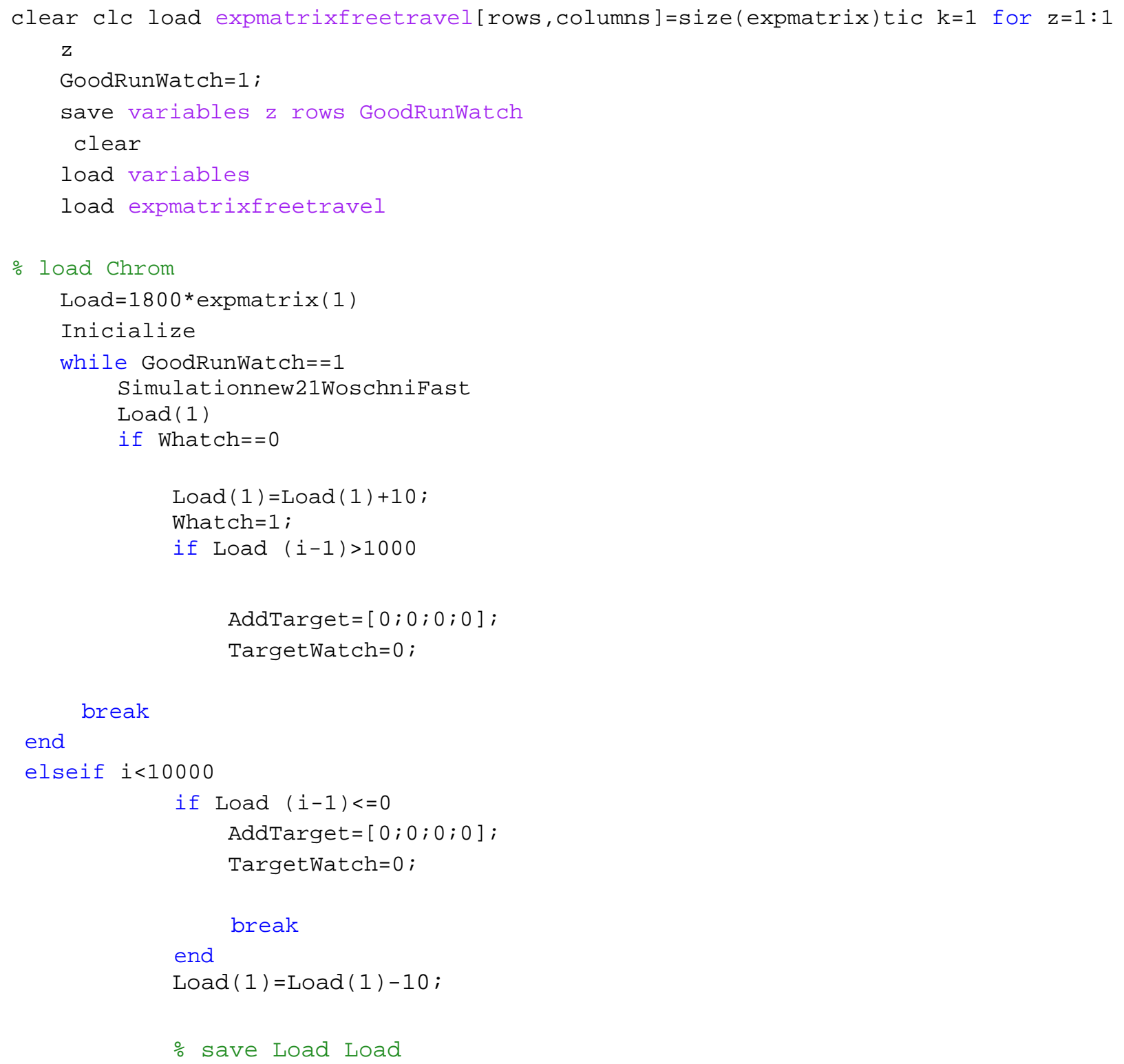




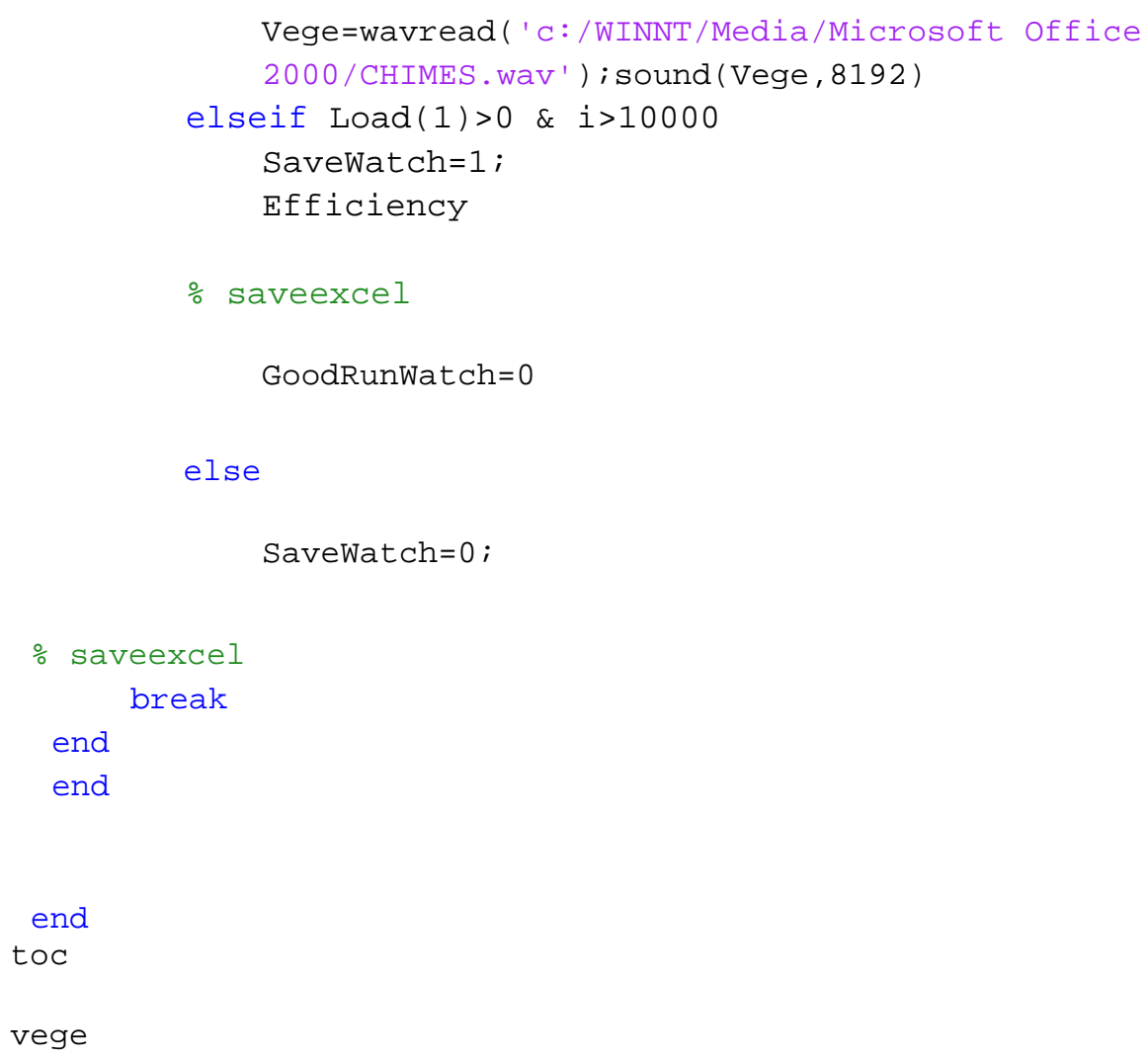

\section{Chrom2NNInput}

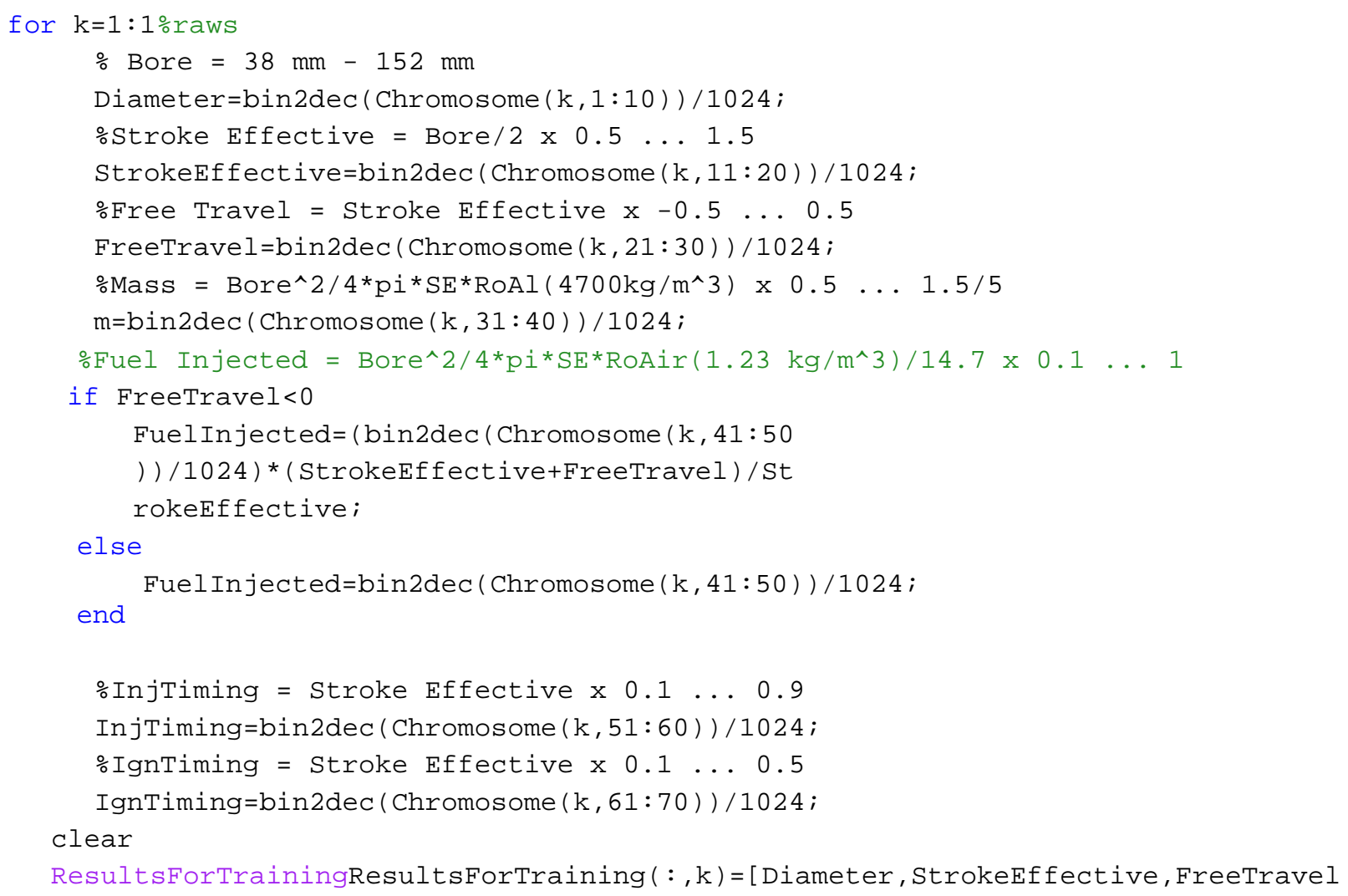




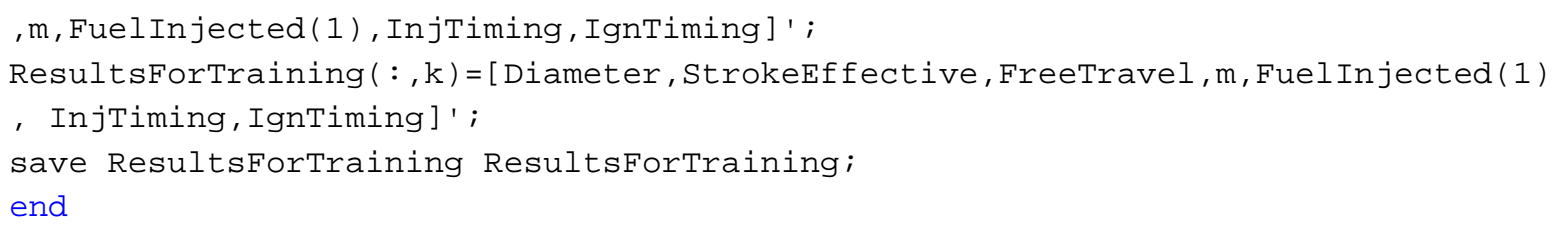

\section{GeneticAlgorithmInitial}

$[\mathrm{mm}, \mathrm{n}]=$ size $($ chromosom $)$;

for $p=1: 1$

\%Reproduction. It makes the four poorest equal to the best and gets them up to thefront.

for $r=1: 5$

poor $=$ find (functionvalue $==\min$ (functionvalue) );

good $=$ find $($ functionvalue $==\max ($ functionvalue $)$ );

$\operatorname{chromosom}(\operatorname{poor}(1),:)=\operatorname{chromosom}(r,:)$;

$\operatorname{chromosom}(r,:)=\operatorname{chromosom}(\operatorname{good}(1),:)$;

functionvalue $(\operatorname{poor}(1))=$ functionvalue $(r)$;

functionvalue $(r)=$ functionvalue $(\operatorname{good}(1))$;

end

for $s=1: 10$

$\mathrm{rr}=$ find (functionvalue $(\mathrm{s}: \mathrm{mm})==\min ($ functionvalue $(\mathrm{s}: \mathrm{mm})))+\mathrm{s}-1$;

$\operatorname{poor}(s)=r r($ length $(r r))$;

$\mathrm{dd}=\mathrm{find}($ functionvalue $(\mathrm{s}: \mathrm{mm})==\max ($ functionvalue $(\mathrm{s}: \mathrm{mm})))+\mathrm{s}-1$;

$\operatorname{good}(s)=d d($ length $(d d))$;

goodchromstore $(s,:)=$ chromosom $(\operatorname{good}(s),:)$;

$\operatorname{chromosom}(\operatorname{good}(s),:)=\operatorname{chromosom}(\operatorname{poor}(s),:)$;

$\operatorname{chromosom}(\operatorname{poor}(s),:)=\operatorname{chromosom}(s,:)$;

$\operatorname{chromosom}(\mathrm{s},:)=$ goodchromstore $(\mathrm{s},:)$;

goodfuncstore $(s)=$ functionvalue $(\operatorname{good}(s))$;

functionvalue $(\operatorname{good}(s))=$ functionvalue $(\operatorname{poor}(s))$;

functionvalue (poor (s))=functionvalue (s);

functionvalue (s)=goodfuncstore (s);

functionvalue(1)

end

for $\mathrm{g}=1: \mathrm{s}$

$\operatorname{chromosom}(\operatorname{good}(g),:)=\operatorname{goodchromstore}(g,:)$;

functionvalue $(\operatorname{good}(\mathrm{g}))=$ goodfuncstore $(\mathrm{g})$;

functionvalue;

end

\%crossover with the Elite. It keeps the two best solution unchanged and crosses random 3 bits in other rows. The two best ones are crossed with each other to keep the blueblood 


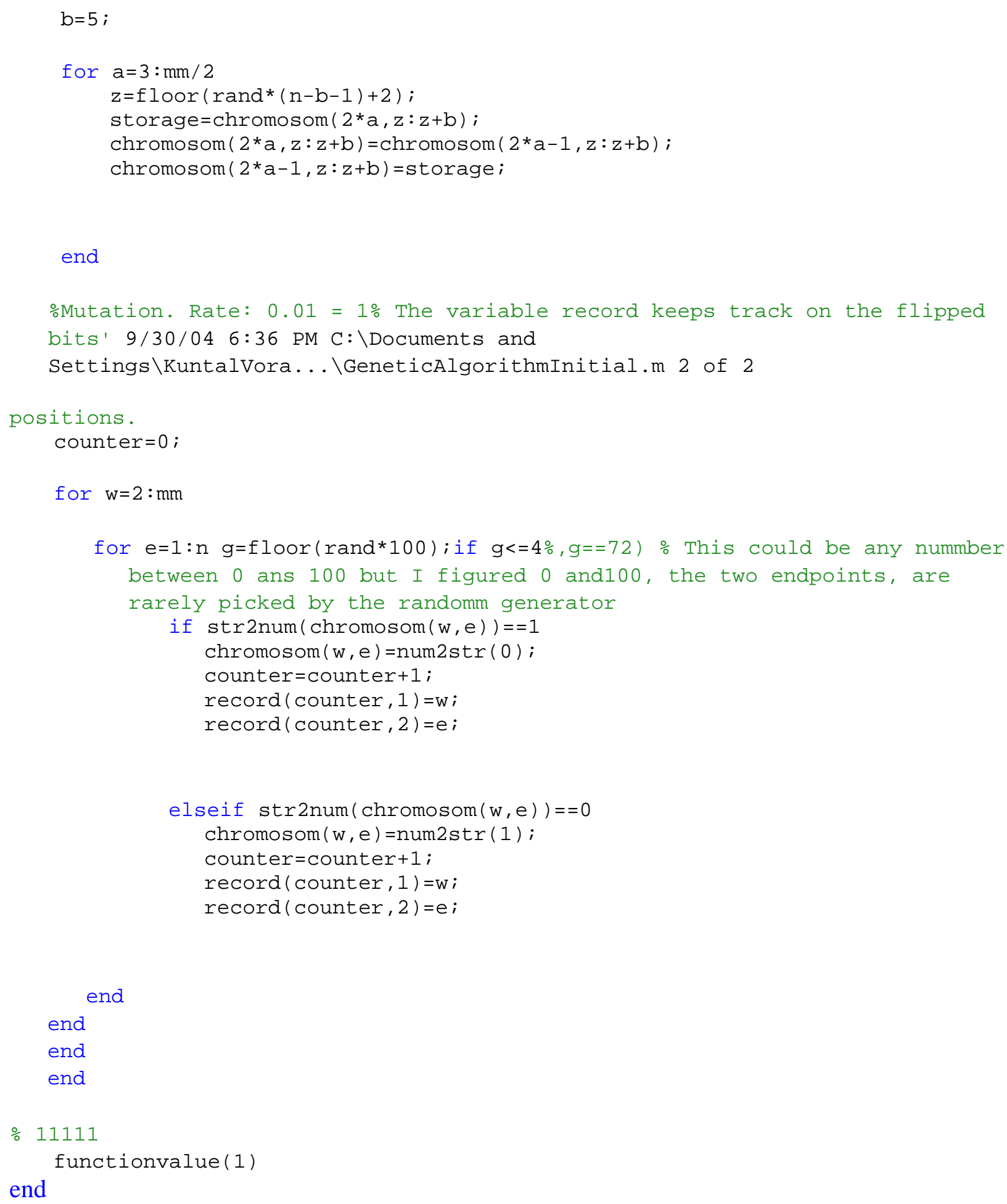

end

end

end

end

\% 11111

functionvalue (1)

end

\section{Inicialize}

$\mathrm{m}=$ expmatrix $(4)$;

Diameter=expmatrix(1);

StrokeEffective=expmatrix(2); 


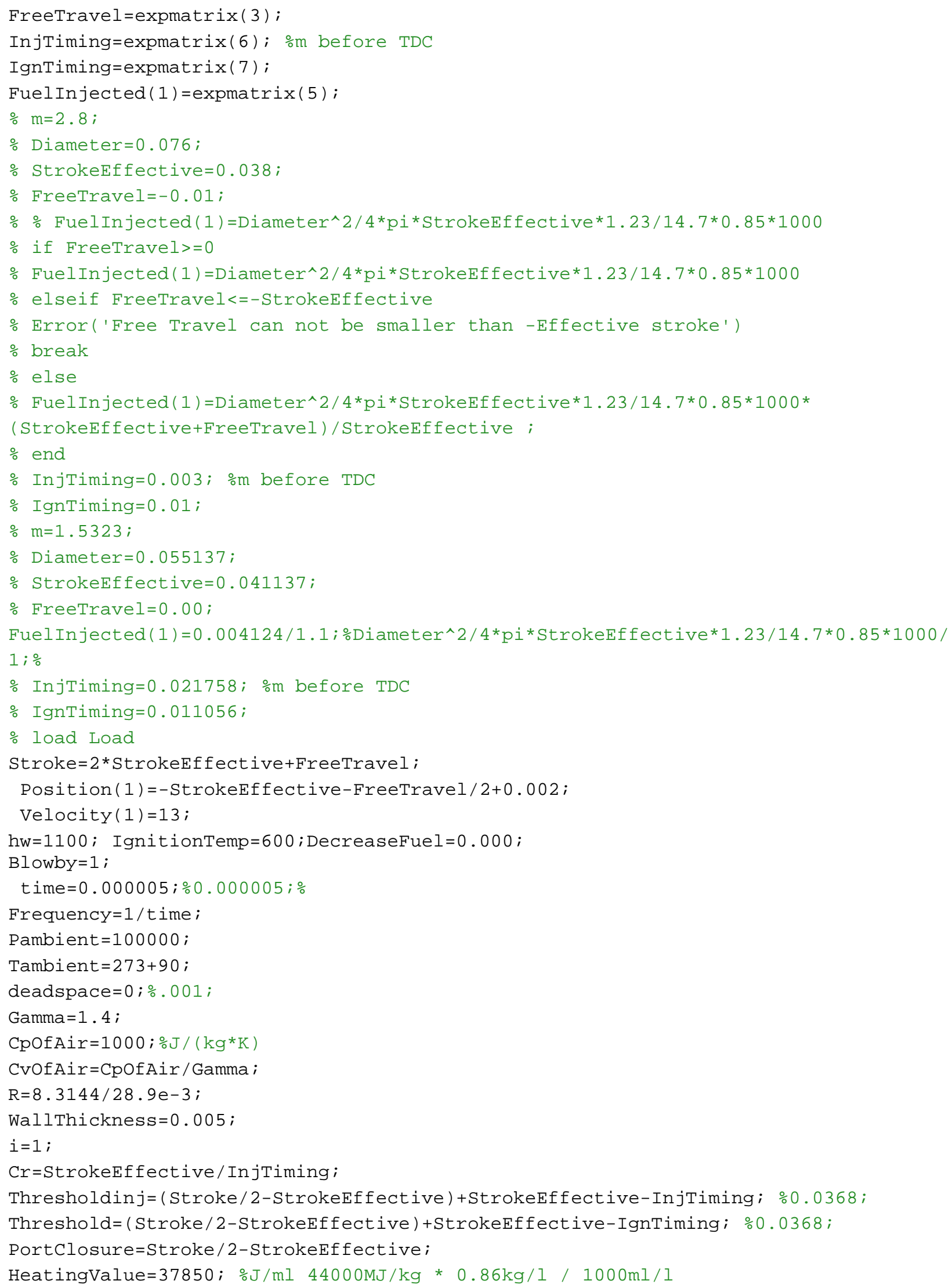




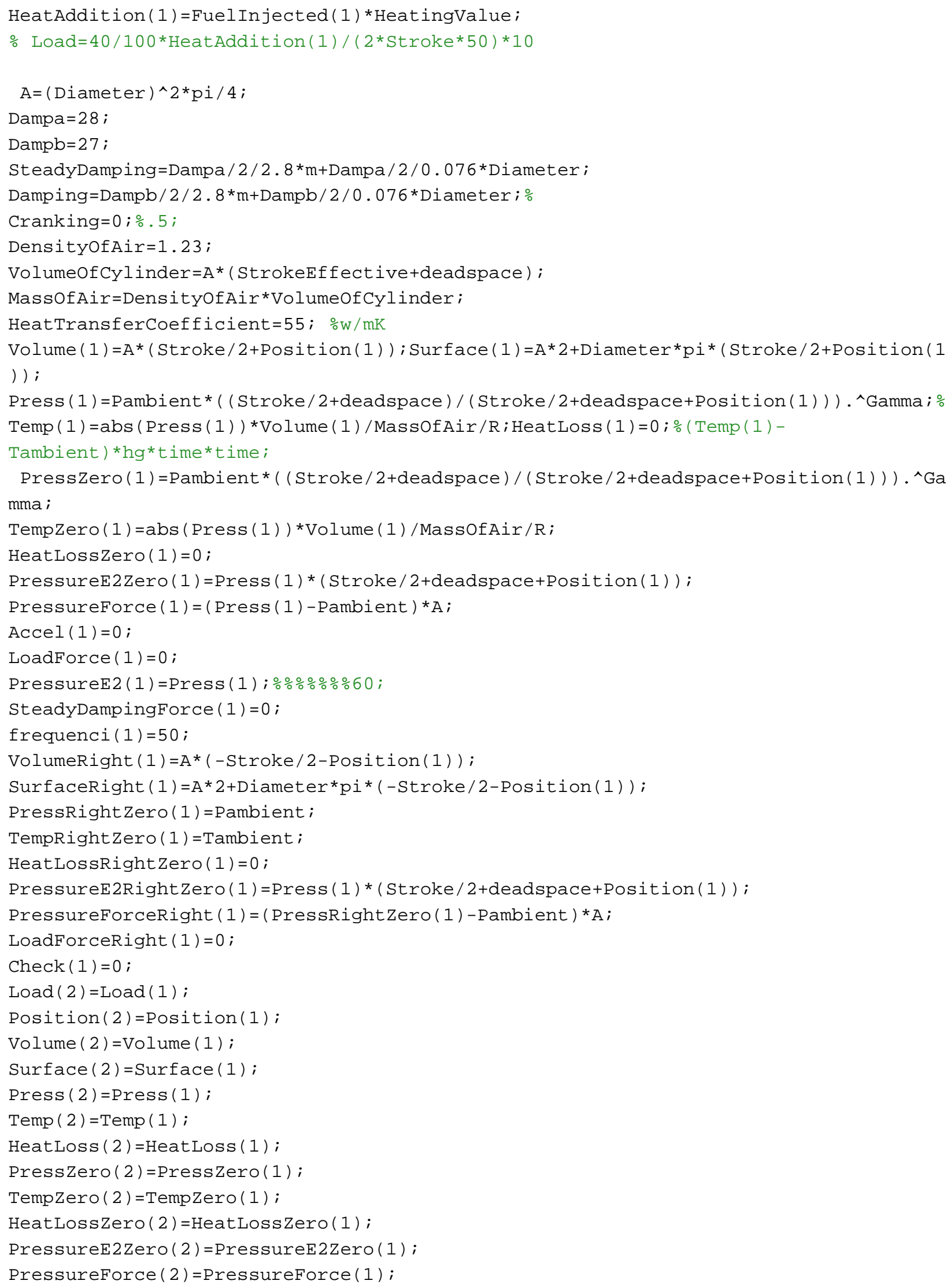




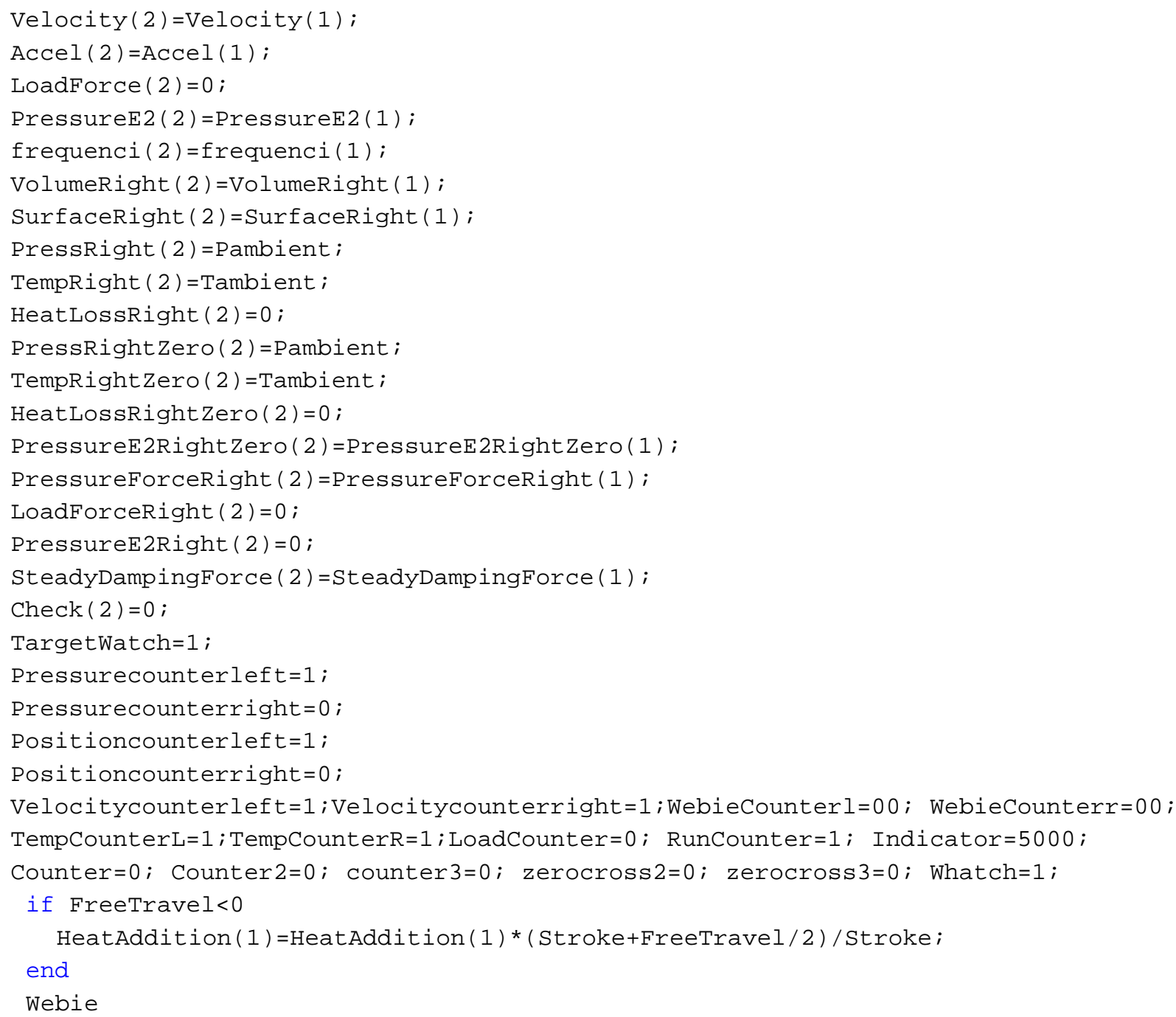

\section{Simulationnew21WoschniFast}

format short $g$

tic

Inicialize

$i=2$;

while Runcounter==1

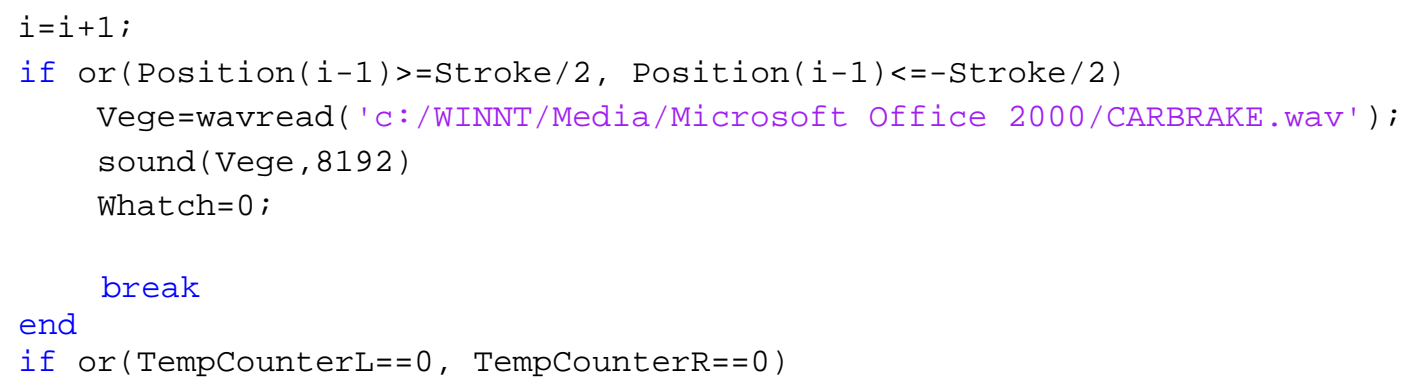




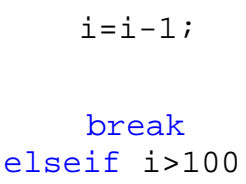

if and(or(and(Position(i-1)<=Position(i-10), Position(i-1)>=Position( $i-$ 100)), and(Position(i-1)>=Position(i-10), Position(i-1)<=Position(i-100))), $\operatorname{not}(\operatorname{or}(\operatorname{Check}(i-1)==1, \operatorname{Check}(i-1)==6))) i=i-1$;

break

end

end

if $\operatorname{ceil}(i / 15000)==i / 15000$

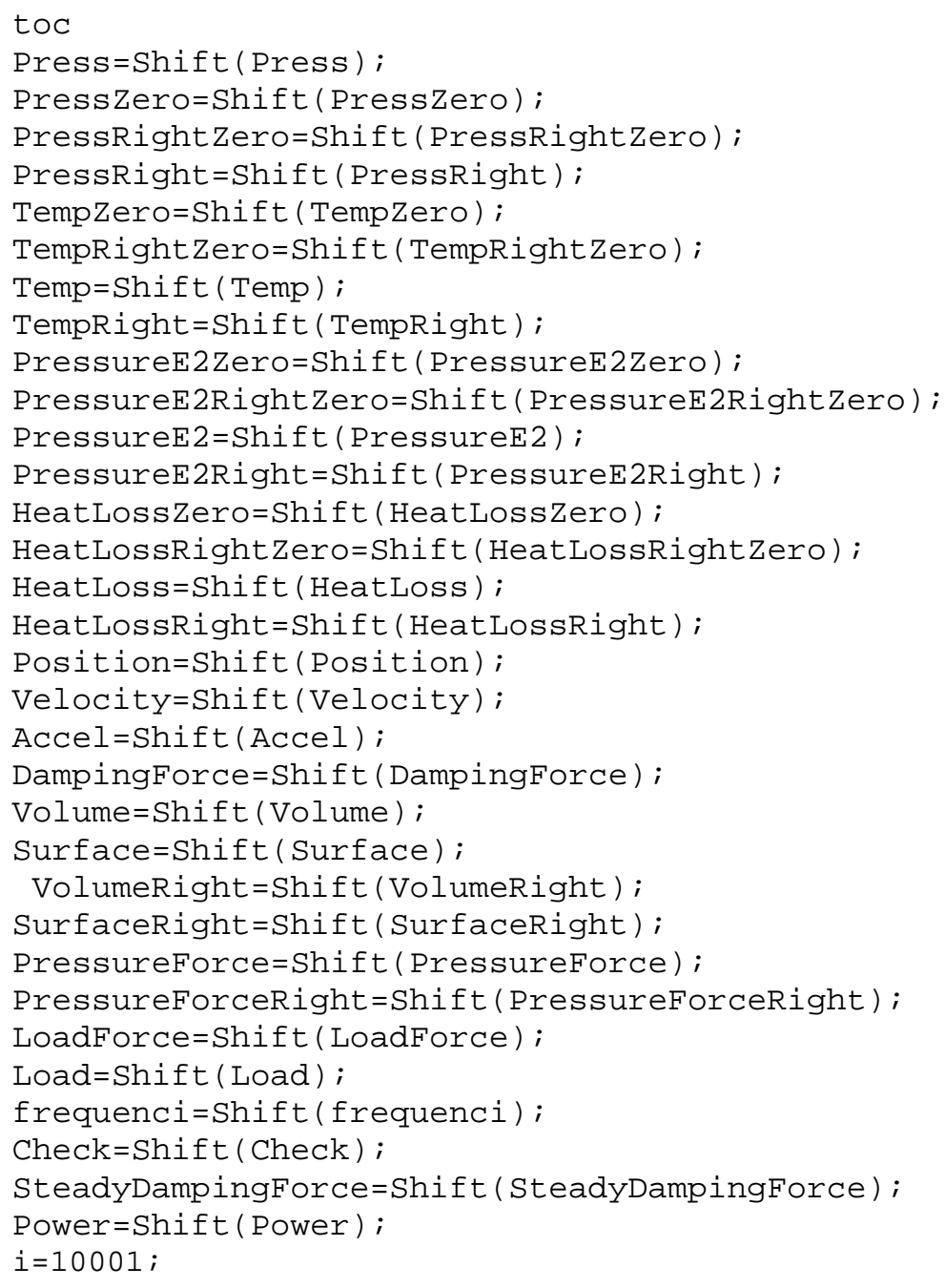




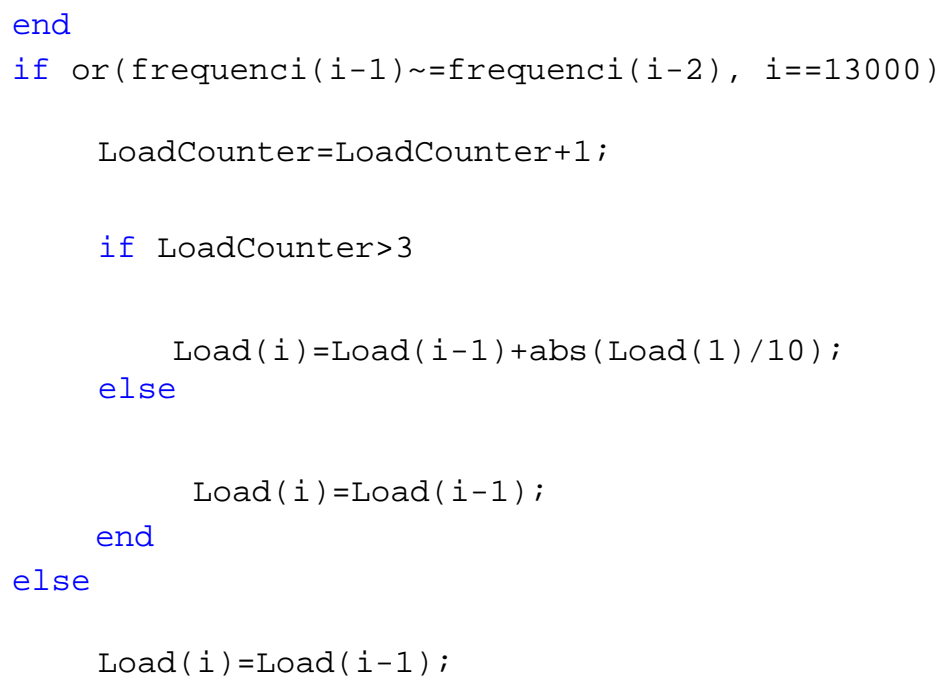

end

Velocity $(i)=\operatorname{Velocity}(i-1)+\operatorname{Accel}(i-1) *$ time; Position(i)=Position(i-

1)+Velocity (i)*time;DampingForce(i)=Damping*Velocity(i);

Volume $(i)=A^{*}($ Stroke/2+Position $(i))$;

Surface $(i)=A^{*} 2+$ Diameter* $\mathrm{pi}^{*}$ (Stroke/2+Position(i)); VolumeRight(i)=A*(Stroke/2-

Position(i)); SurfaceRight(i)=A*2+Diameter *pi*(Stroke/2-Position(i));

if or ( (Position(i)<-Threshold \& Temp(i-1)>=IgnitionTemp \&

Positioncounterleft $==0$ \&WebieCounterl<length $(\mathrm{Q}(:))), \quad(($ WebieCounterl<length $(\mathrm{Q}(:))$

\& Webiecounterl>0) \& Position(i)<=-(Stroke/2-StrokeEffective) \& Position(i)<=0));\%

\& Position(i)<(-StrokeEffective-FreeTravel)))

RunCounter $=1$; WebieCounterl=WebieCounterl+1;

Positioncounterright $=0$; Positioncounterleft $=1$; Pressurecounterright $==0$;

WebieCounter $r=0$; $\operatorname{Check}(i)=1$;

$[\operatorname{PressZero}(i), \operatorname{TempZero}(i), \operatorname{PressureE2Zero}(i), \operatorname{HeatLossZero}(i)]=$ Calc(StrokeEffective, Stroke, Position(i), Position(i-1), Volume(i-1), Presszero(i1), PressZero(i-1), TempZero(i-1), Surface(i), Volume(i), PressureE2Zero(i$1)$, Webiecounter 1,0 , time, frequenci(i-1), Diameter );

[PressRightZero(i), TempRightZero(i), PressureE2RightZero(i), HeatLossRightz ero(i)] =CalcZero(Tambient, Pambient);

$[\operatorname{Press}(i), \operatorname{Temp}(i), \operatorname{PressureE2}(i), \operatorname{HeatLoss}(i)]=$ Calc(StrokeEffective, Stroke, Position(i), Position(i-1), Volume(i-1), Press(i1), PressZero(i-1), Temp(i-1), Surface(i), Volume(i), PressureE2(i-

1), WebieCounterl, Q(WebieCounterl), time, frequenci( $i$ -

1), Diameter); [PressRight (i), TempRight(i), PressureE2Right(i), HeatLossRight(

i) ] = CalcZero(Tambient, Pambient);

$\operatorname{ccc}=1$;

elseif or ( (Position(i)>Threshold \& TempRight(i-1)>=IgnitionTemp \&Positioncounterright $==\odot$ \& WebieCounterr $<$ length $(Q(:)))$, 
((WebieCounterr<length $(\mathrm{Q}(:))$ \& WebieCounter $r>0)$ \& Position(i)>=(Stroke/2StrokeEffective) \& Position $(i)>=0))$;

RunCounter $=1$; WebieCounter $r=$ WebieCounter $r+1$;

Positioncounterright $=1$; Positioncounterleft $=0$; WebieCounterl $=0$;

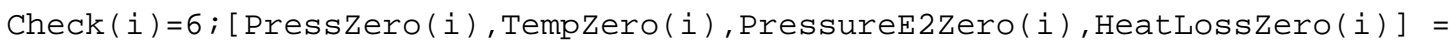
CalcZero(Tambient, Pambient);

[PressRightZero(i), TempRightZero(i), PressureE2RightZero(i), HeatLossRightZer $o(i)]=C a l c$ (StrokeEffective, Stroke,Position(i), Position(i-1), VolumeRight (i1), PressRightZero(i-1), PressRightZero(i-1), TempRightZero(i-1), SurfaceRight(i), VolumeRight (i), PressureE2RightZero(i-1), webiecounterr, $\odot$, time, frequenci(i1), Diameter);

[Press(i), Temp(i),PressureE2(i), HeatLoss(i)] = CalcZero(Tambient,Pambient);

[PressRight (i), TempRight (i), PressureE2Right (i), HeatLossRight (i)] $=$ Calc (StrokeEffective, Stroke,Position(i), Position(i-1), VolumeRight ( $i$ 1), PressRight (i-1), PressRightZero(i-1), TempRight(i-1), SurfaceRight (i), VolumeRight(i), PressureE2Right(i-1), Webiecounterr, Q(WebieCounterr), time, frequenci(i-1), Diameter);

$c c c=1$; elseif or ((Position(i)>=PortClosure \& Position(i)<=-Portclosure), (Position( $i)<=$ PortClosure \& Position(i) $>=-$ PortClosure)) if FreeTravel $>=0$ Pressurecounterleft $=1$; Positioncounterleft $=0$; WebieCounter $r=0$; WebieCounterl $=0$; Check(i) $=3$; [PressRightZero(i), TempRightZero(i), PressureE2RightZero(i), HeatLossRightZero(i)] = CalcZero(Tambient, Pambient); [PressZero(i), TempZero(i), PressureE2Zero(i), HeatLossZe ro(i)] = CalcZero(Tambient, Pambient); [PressRight (i), TempRight (i), PressureE2Right $(i)$, HeatLossRight $(i)]$ = CalcZero(Tambient, Pambient);

[Press(i), Temp(i), PressureE2(i), HeatLoss(i)] = CalcZero (Tambient, Pambient); ccc=1;

else Pressurecounterleft $=1$;

if (WebieCounterl<length $(Q(:))$ \& WebieCounterl>0 \& Position(i)<=(Stroke/2-StrokeEffective))

WebieCounterl=WebieCounterl+1; $\operatorname{Check}(i)=1.5$; [PressZero(i), TempZero(i), PressureE2Zero(i), HeatLossZero(i)] = Calc(StrokeEffective, Stroke,Position(i), Position(i-1), Volume(i-1), PressZero(i-1), PressZero(i-1), TempZero(i-1), Surface(i), Volume(i), PressureE2Zero(i-1), Webiecounterl, $\odot$, time, frequenci(i-1), Diameter);

$[\operatorname{Press}(i), \operatorname{Temp}(i), \operatorname{PressureE2}(i), \operatorname{HeatLoss}(i)]=$ Calc(StrokeEffective, Stroke, Position(i), Position(i-1), Volume(i1), Press(i-1), PressZero(i-1), Temp(i-1), Surface(i), Volume(i), PressureE2(i-1), webieCounterl, Q(Webiecounterl), time, frequenci(i-1), Diameter); [PressRightZero(i), TempRightZero(i), PressureE2RightZero( 


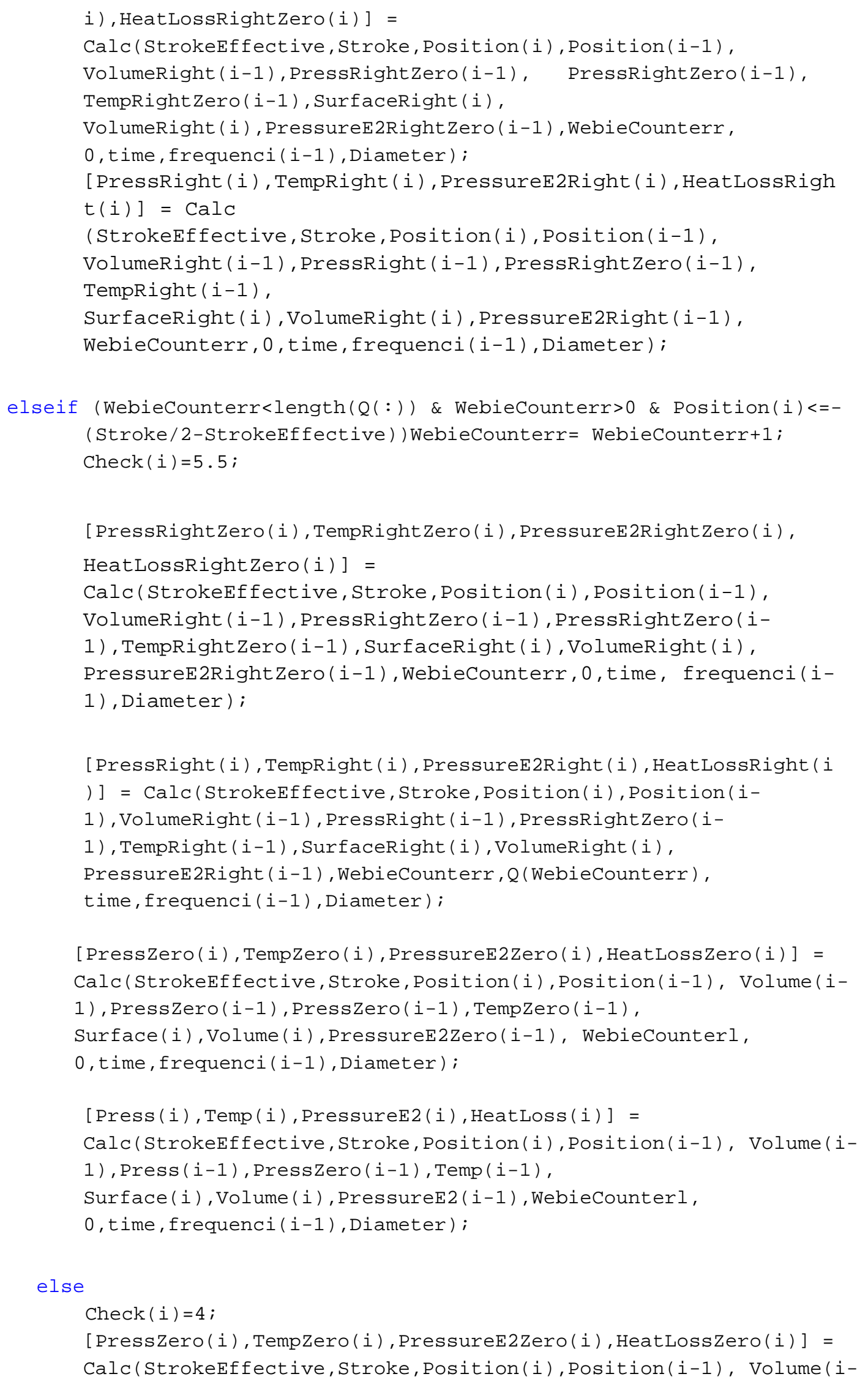


1), PressZero(i-1), PressZero(i-1), TempZero(i-1), Surface (i), Volume(i), PressureE2Zero(i-1), Webiecounter 1,0 , time, frequenci(i-1), Diameter);

[PressRightZero(i), TempRightZero(i),PressureE2RightZero(i), HeatLo ssRightZero(i)] = Calc(StrokeEffective, Stroke,Position(i),Position(i-1), VolumeRight (i-1), PressRightZero(i-1), PressRightZero(i-1), TempRightZero(i-1), SurfaceRight(i), VolumeRight(i), PressureE2RightZero(i-1), Webiecounterr, 0 , time, frequenci ( $i$ 1), Diameter);

$[\operatorname{Press}(i), \operatorname{Temp}(i), \operatorname{PressureE2}(i), \operatorname{HeatLoss}(i)]=$ Calc(StrokeEffective, Stroke,Position(i),Position(i-1), Volume(i1), $\operatorname{Press}(i-1), \operatorname{PressZero}(i-1), \operatorname{Temp}(i-1), \operatorname{Surface}(i)$, Volume(i), PressureE2(i-1), webiecounterl, $\odot$, time, frequenci(i 1), Diameter);

[PressRight(i), TempRight (i), PressureE2Right(i), HeatLossRight (i )$]=$ Calc (StrokeEffective, Stroke,Position(i),Position(i-1), VolumeRight (i-1), PressRight (i-1), PressRightZero(i-1), TempRight (i-1), SurfaceRight (i), VolumeRight (i), PressureE2Right (i-1), webiecounter, 0 , time, frequenci $(i-$ 1), Diameter ); end

$\operatorname{ccc}=1$;

if Webiecounter $r==$ length $(Q(:))$ Webiecounter $r=0$;

end

if Webiecounterl==length $(\mathrm{Q}(:))$ Webiecounter $1=0$;

end

end

else

if Position(i) $<0$

if Position(i)>Position(i-1)\&

Position(i-1)<Position(i-2)

Runcounter $=0$;

end

Pressurecounterleft $=1$; Pressurecounterright $=0$;

Check(i)=2; [PressZero(i), TempZero(i), PressureE2Zero(i), HeatLossZero(i)] = Calc(StrokeEffective, Stroke,Position(i),Position(i-1), Volume(i1), PressZero(i-1), PressZero(i-1), TempZero(i-1), Surface(i), Volume(i), PressureE2Zero(i-1), Webiecounterl, 0 , time,frequenci(i-1), Diameter); [PressRightZero(i), TempRightZero(i), PressureE2RightZero(i), HeatLos sRightZero(i)] = CalcZero(Tambient, Pambient); 


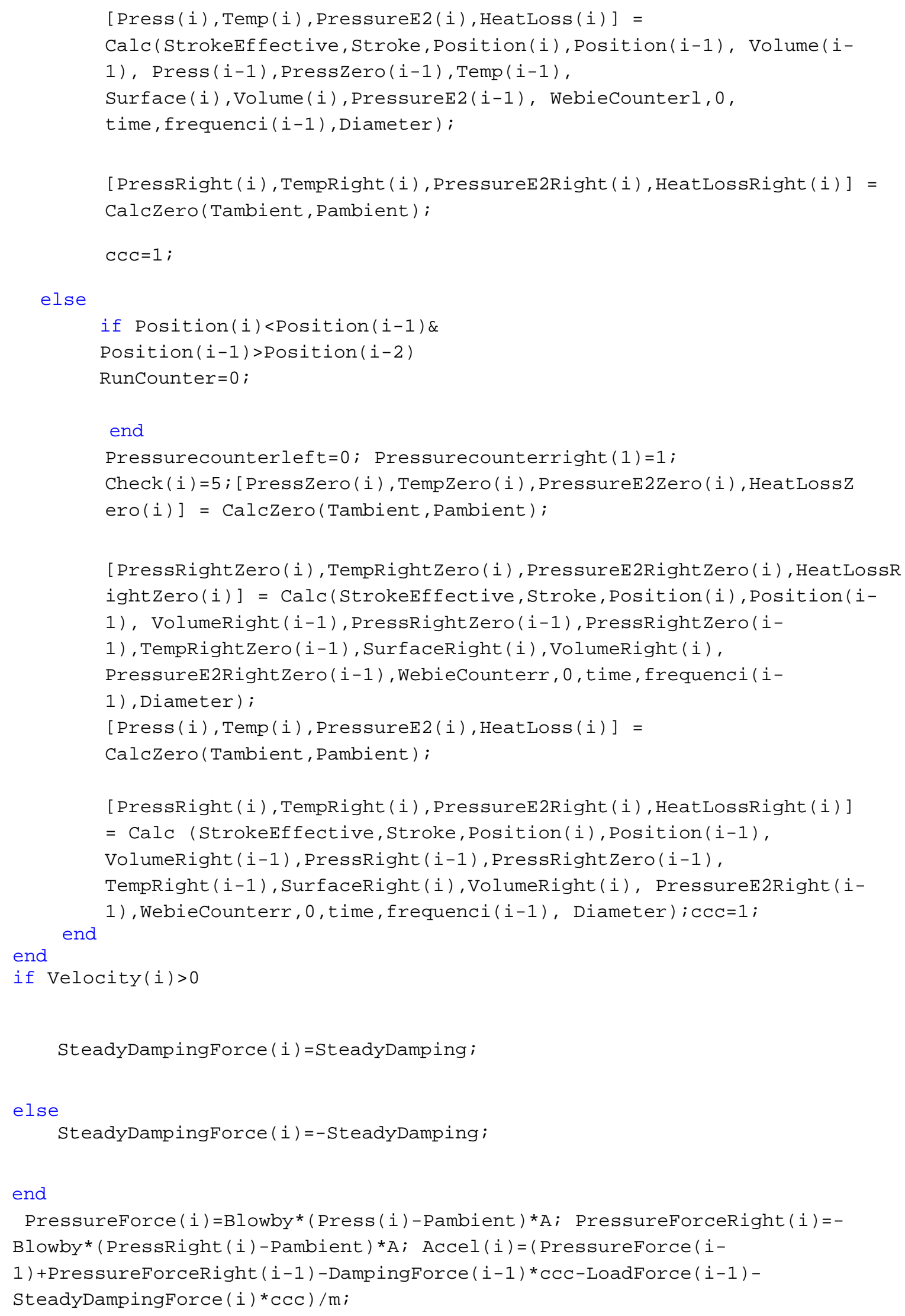




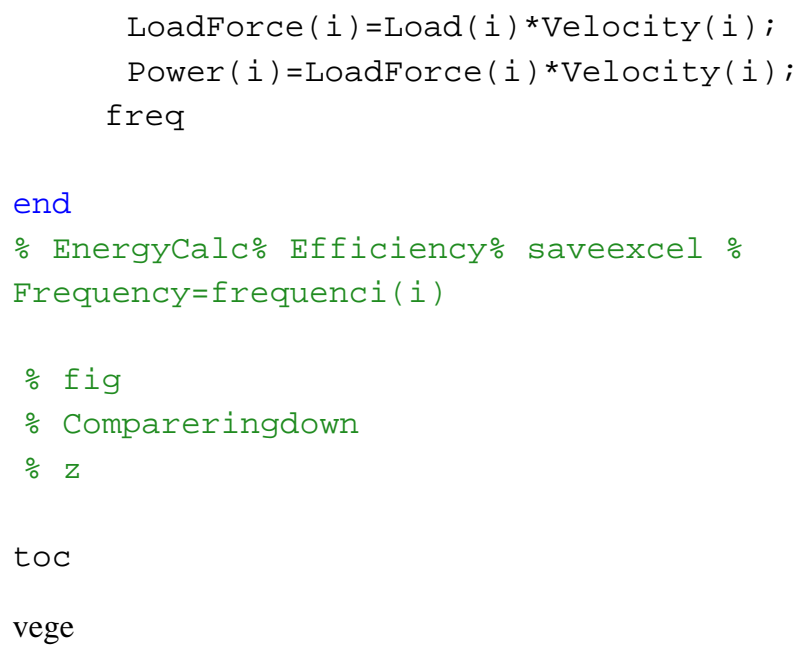

\section{Efficiency}

$\mathrm{CR}=$ StrokeEffective/(StrokeEffective-(StrokeEffective-Stroke/2+abs(Position(i-1)))) Frequenci=frequenci(i-1) \%Hz

$\%$ for $d=z e r o c r o s s 3+1:$ zerocross 1

$\% \% p(j$-zerocross 3$)=\operatorname{Load}(i-100) * \operatorname{Velocity}(j)^{\wedge} 2^{*}$ time ;

$\% p(d-z e r o c r o s s 3)=\operatorname{Load}(i-100) * \operatorname{Velocity}(d) \wedge 2 *$ time;

$\%$ end

$\%$ power $=\operatorname{sum}(\operatorname{abs}(p)) * 2 *$ Frequenci

$\mathrm{P}=\operatorname{abs}\left(\operatorname{Load}(i-100) *\left(2 *\right.\right.$ Stroke ${ }^{*}$ Frequenci $\left.)\right) * 2 *$ Stroke ${ }^{*}$ Frequenci

Efficiency $=\mathrm{P} /($ HeatAddition $(1) * 2 *$ Frequenci $) * 100$

$\mathrm{PPP}=\operatorname{sum}($ Power (zerocross $3+1$ : zerocross 1$)) *$ time

Efficienc $=$ PPP $/($ HeatAddition $(1)) * 100$

\section{saveexcel}

load filecounter

savefile = sprintf('ResultsFreeTravelForNN\%d' , filecounter -1$)$;

savefilechrom $=\operatorname{sprintf('ChromResultsForNN\% d',filecounter-1)}$;

savefileTarget $=\operatorname{sprintf}($ 'TargetResultsForTraining\%d', filecounter -1$) ;$

savefileNN = sprintf('NNperformanceandprediction\%d', filecounter-1);

load (savefileNN)

load (savefilechrom)

load (savefileTarget)

load(savefile)

load Preformance

load NNPredEfficiency

load Chrom

load FinalResults

Addition=[Diameter, StrokeEffective,FreeTravel, m, FuelInjected(1), InjTiming, IgnTimin g, Load (i-9), CR, Frequenci, Efficienc, P, filecounter ]; 


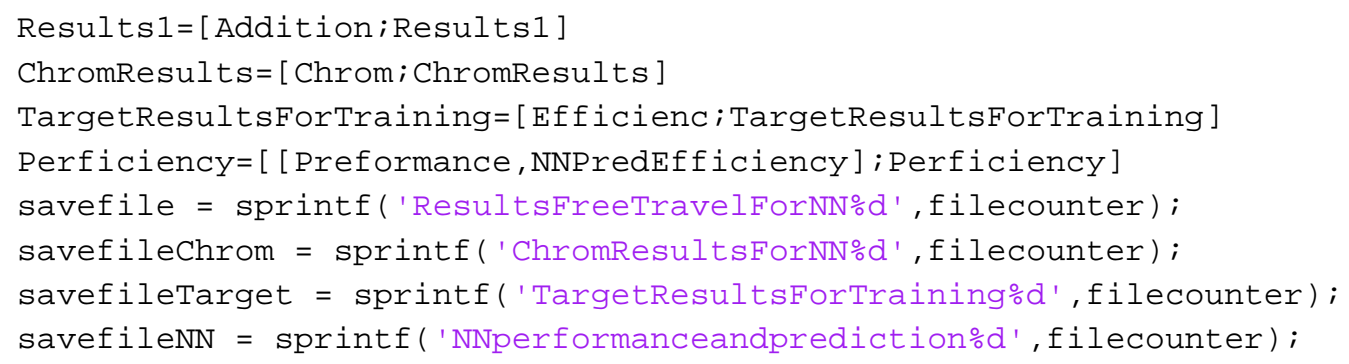

[raws, columns]=size(Results1);

FinalResults=Results1 $(:, 11)$;

save FinalResults FinalResults

filecounter $=$ raws +1 ;

save savefile

save (savefile, 'Results1')

save (savefilechrom, 'ChromResults')

save (savefileTarget, 'TargetResultsForTraining')

save (savefilenN, 'Perficiency')

save filecounter filecounter

save Results12forTraining Results1

\section{Webie}

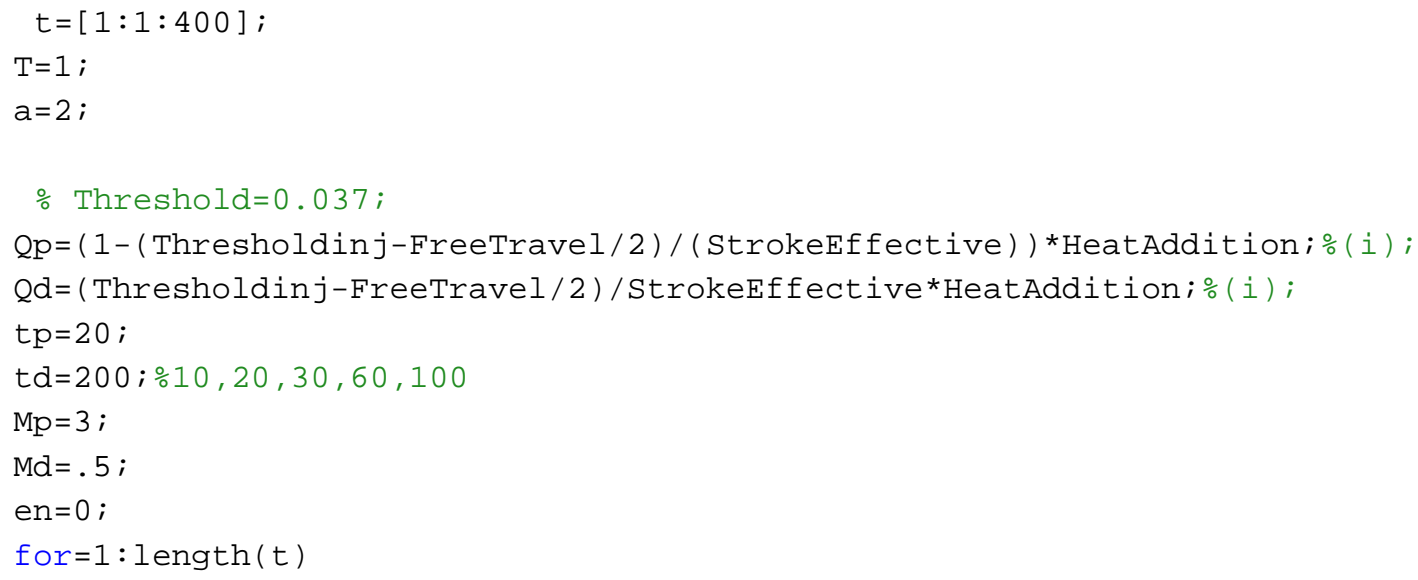

\section{Calc}

function [Press, Temp, PressEnergy, HeatLoss] = Calc(StrokeEffective, Stroke, Position, 


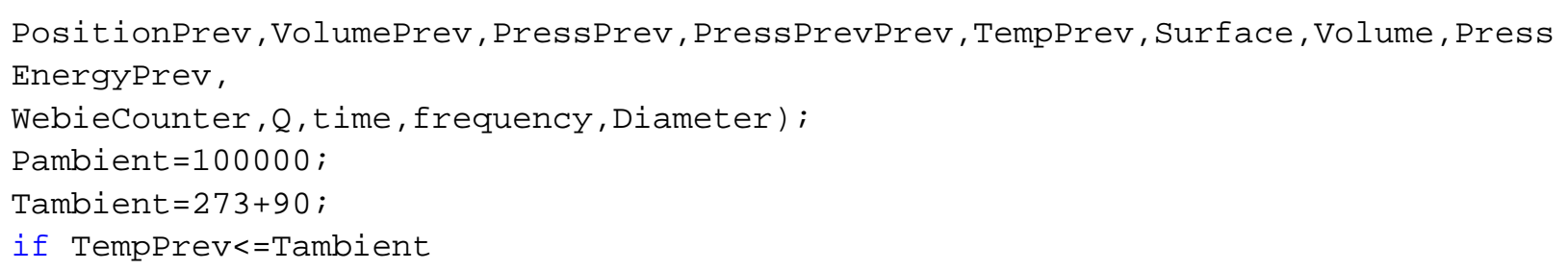




\section{CalcZero}

function [Press,Temp,PressEnergy,HeatLoss] = CalcZero(Tambient,Pambient);

$\mathrm{cc}=0$;

hg=0;

HeatResistance $=0$;

HeatLoss $=0$;

$\mathrm{Dp}=0$;

Press=Pambient;

Temp=Tambient;

PressEnergy=0;

\section{Shift}

function [Press]=Shift(Press)

Temporary=Press(5000:14999);

clear Press

Press=Temporary;

\section{Freq}

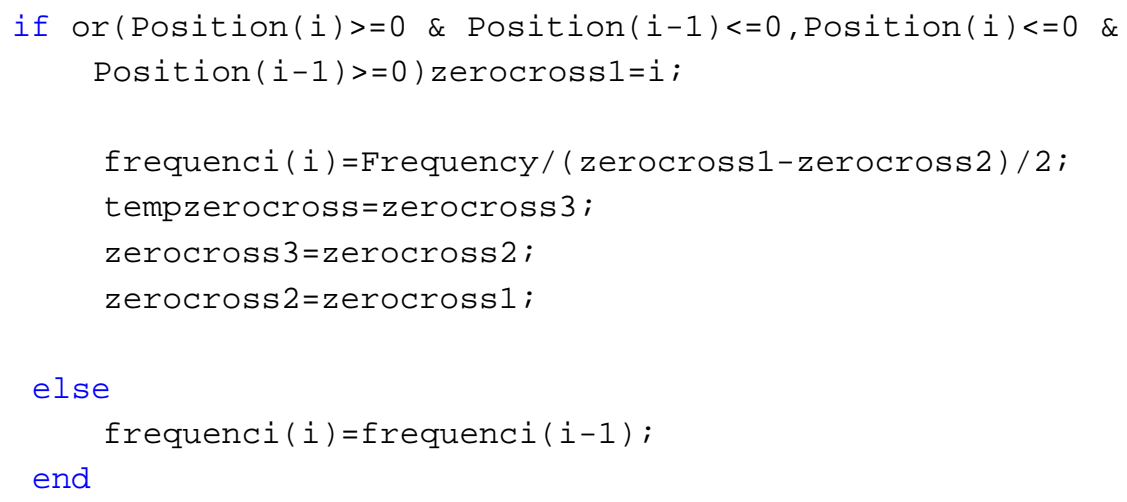

\section{Chrom2NNInputForNeural}

\% load ChromResultsFornN51

[raws, columns]=size (ChromResults)

Chromosome $=$ ChromResults;

for $\mathrm{k}=1$ : raws

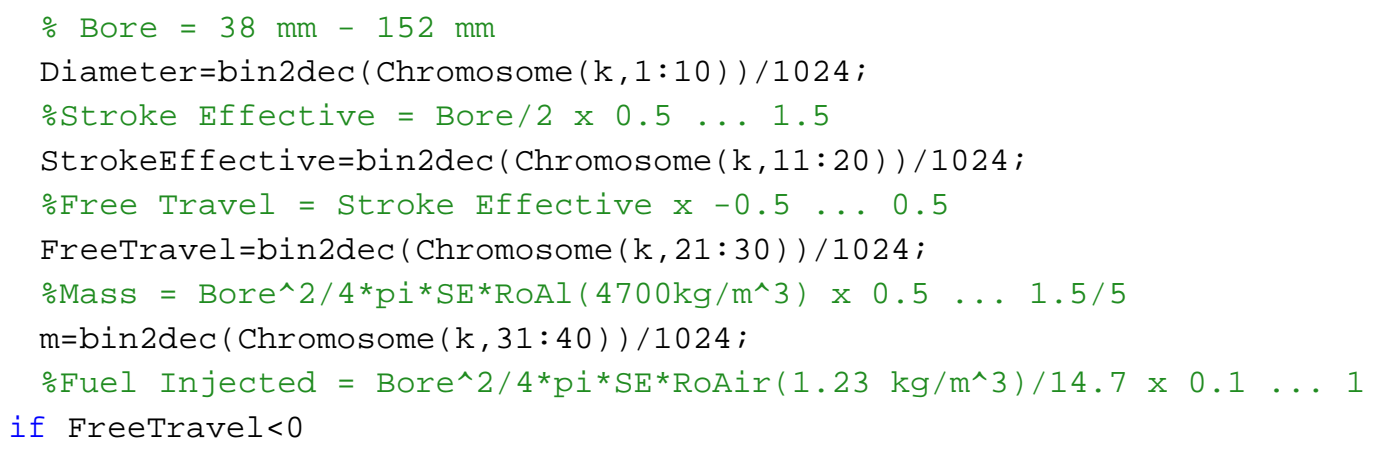




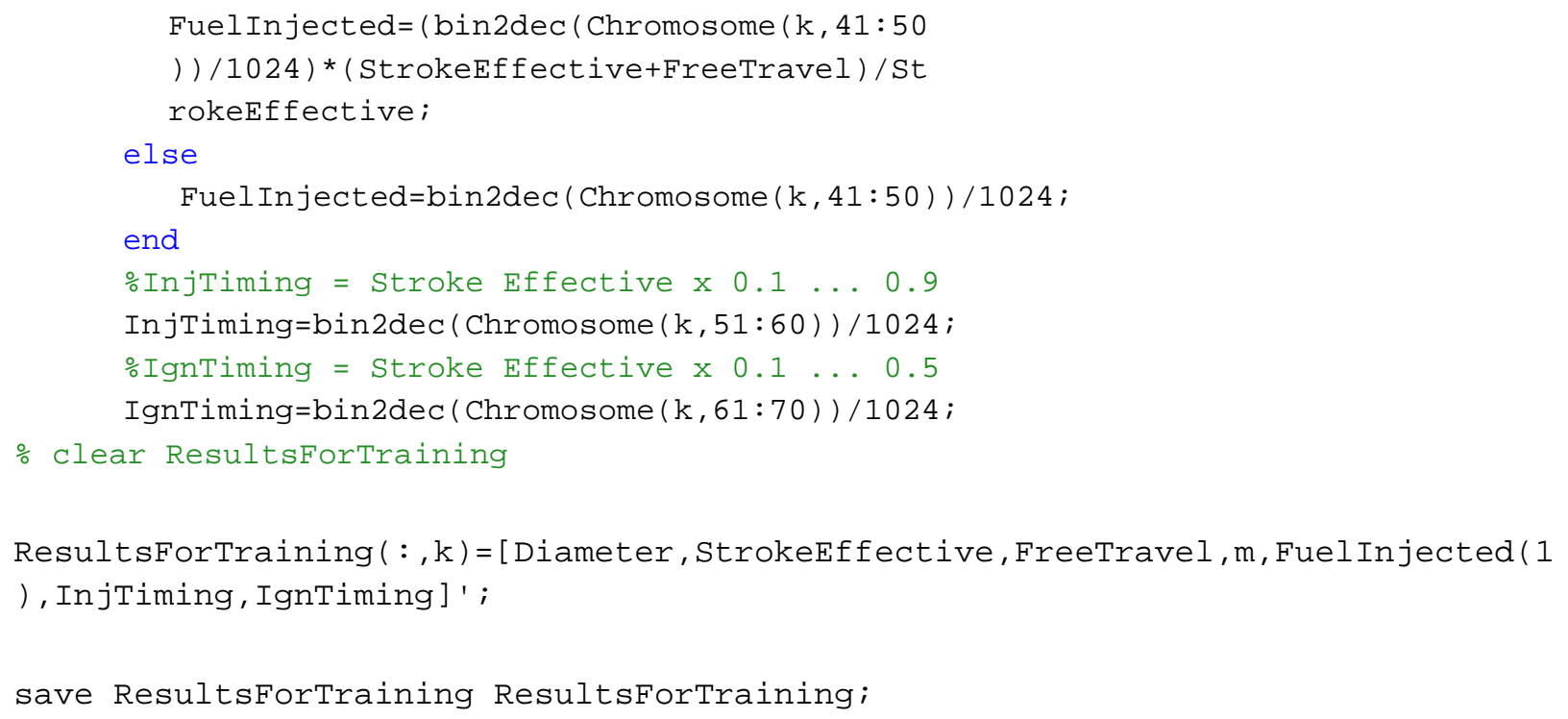

